\title{
Channel Modeling and Performance of Zigbee Radios in an Industrial Environment
}

\author{
Jeff Peil \\ Mehrdad Damsaz \\ Derek Guo \\ Wayne Stark \\ Richard Candell \\ Nader Moayeri
}

This publication is available free of charge from:

https://doi.org/10.6028/NIST.TN.1941

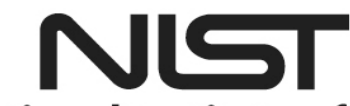

National Institute of Standards and Technology U.S. Department of Commerce 


\title{
Channel Modeling and Performance of Zigbee Radios in an Industrial Environment
}

\author{
Jeff Peil \\ Mehrdad Damsaz \\ Derek Guo \\ Wayne Stark \\ Electrical Engineering and Computer Science Department \\ The University of Michigan \\ Richard Candell \\ Intelligent Systems Division \\ Engineering Laboratory \\ Nader Moayeri \\ Advanced Network Technologies Division \\ Information Technology Laboratory
}

This publication is available free of charge from: https://doi.org/10.6028/NIST.TN.1941

December 2016

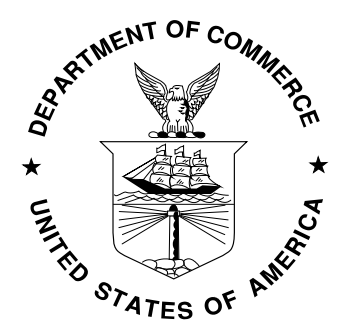

U.S. Department of Commerce Penny Pritzker, Secretary

National Institute of Standards and Technology Willie May, Under Secretary of Commerce for Standards and Technology and Director 
Certain commercial entities, equipment, or materials may be identified in this document in order to describe an experimental procedure or concept adequately. Such identification is not intended to imply recommendation or endorsement by the National Institute of Standards and Technology, nor is it intended to imply that the entities, materials, or equipment are necessarily the best available for the purpose.

National Institute of Standards and Technology Technical Note 1941 Natl. Inst. Stand. Technol. Tech. Note 1941, 190 pages (December 2016) CODEN: NTNOEF

This publication is available free of charge from: https://doi.org/10.6028/NIST.TN.1941 
Final Report for NIST: Award 70NANB14H316

\title{
Channel Modeling and Performance of Zigbee Radios in an Industrial Environment
}

\author{
Jeff Peil, Mehrdad Damsaz, Derek Guo, Wayne Stark, \\ Richard Candell, Nader Moayeri
}

September 1, 2016 


\section{Contents}

1 Introduction $\quad 4$

2 Measurements 4

3 Received Power Channel Models 13

4 Impulse Response $\quad 15$

5 Zigbee Performance $\quad 17$

6 Conclusion $\quad 20$

A Received Power Data: Gaithersburg $\quad 24$

A.1 Cross Polarization, 2.245GHz, Transmitter 1 . . . . . . . . . . . 25

A.2 Cross Polarization, 5GHz, Transmitter $1 \ldots \ldots . \ldots . . \ldots . . \ldots 29$

A.3 Cross Polarization, 2.245GHz, Transmitter 2, High . . . . . . . . . . . 33

A.4 Cross Polarization, 2.245GHz, Transmitter 2, Low . . . . . . . . . . . 37

A.5 Cross Polarization, 5GHz, Transmitter 2, High . . . . . . . . . . . . 41

A.6 Cross Polarization, 5GHz, Transmitter 2, Low . . . . . . . . . . . . 45

A.7 Long Polarization, 2.245GHz, Transmitter $1 \ldots \ldots$. . . . . . . 49

A.8 Long Polarization, $5 \mathrm{GHz}$, Transmitter $1 \ldots \ldots \ldots$

A.9 Long Polarization, 2.245GHz, Transmitter 2, High _ . . . . . . . . . 57

A.10 Long Polarization, 2.245GHz, Transmitter 2, Low . . . . . . . . . . . . 61

A.11 Long Polarization, 5GHz, Transmitter 2, High . . . . . . . . . . . . 65

A.12 Long Polarization, 5GHz, Transmitter 2, Low . . . . . . . . . . . . . 69

A.13 Vertical Polarization, 2.245GHz, Transmitter $1 \ldots \ldots$. . . . . . 73

A.14 Vertical Polarization, 5GHz, Transmitter 1 . . . . . . . . . . 77

A.15 Vertical Polarization, 2.245GHz, Transmitter 2, High . . . . . . . . . . 81

A.16 Vertical Polarization, 2.245GHz, Transmitter 2, Low . . . . . . . . . . 85

A.17 Vertical Polarization, 5GHz, Transmitter 2, High . . . . . . . . . . . 89

A.18 Vertical Polarization, 5GHz, Transmitter 2, Low . . . . . . . . . . . 93

A.19 Channel Parameters Summary . . . . . . . . . . . . . . . . . . . 97

A.20 2.245GHz, Transmitter 1 . . . . . . . . . . . . . . . . 102

A.21 5.4GHz, Transmitter $1 \ldots \ldots \ldots \ldots$. . . . . . . . . . . 104

A.22 2.245GHz, Transmitter $2 \ldots \ldots \ldots \ldots$. . . . . . . . . 106

A.23 5.4GHz, Transmitter $2 \ldots \ldots \ldots \ldots$

B Received Power Data: Automotive Assembly 111

B.1 Horizontal Polarization, 2.245GHz, Transmitter 1 Outer Path . . . . . . 111

B.2 Horizontal Polarization, 5.4GHz, Transmitter 1, Outer Path . . . . . . . 115

B.3 Horizontal Polarization, 2.245GHz, Transmitter 1, Inner Path . . . . . . 119

B.4 Horizontal Polarization, 5.4GHz, Transmitter 1, Inner Path . . . . . . . 123 
B.5 Horizontal Polarization, 2.245GHz, Transmitter 2 Outer Path . . . . . . 127

B.6 Horizontal Polarization, 5.4GHz, Transmitter 2, Outer Path . . . . . . . 131

B.7 Horizontal Polarization, 2.245GHz, Transmitter 2, Inner Path . . . . . . 135

B.8 Horizontal Polarization, 5.4GHz, Transmitter 2, Inner Path . . . . . . . 139

B.9 Vertical Polarization, 2.245GHz, Transmitter 1 Outer Path . . . . . . . . 143

B.10 Vertical Polarization, 5.4GHz, Transmitter 1, Outer Path . . . . . . . . 147

B.11 Vertical Polarization, 2.245GHz, Transmitter 1, Inner Path . . . . . . . 151

B.12 Vertical Polarization, 5.4GHz, Transmitter 1, Inner Path . . . . . . . . . 155

B.13 Vertical Polarization, 2.245GHz, Transmitter 2 Outer Path . . . . . . . . 159

B.14 Vertical Polarization, 5.4GHz, Transmitter 2, Outer Path . . . . . . . . 163

B.15 Vertical Polarization, 2.245GHz, Transmitter 2, Inner Path . . . . . . . 167

B.16 Vertical Polarization, 5.4GHz, Transmitter 2, Inner Path . . . . . . . . . 171

B.17 Channel Parameters Summary . . . . . . . . . . . . . . . . . 175

B.18 2.245GHz, Transmitter 1 . . . . . . . . . . . . . . . . . . 179

B.19 5.4GHz, Transmitter $1 \ldots \ldots \ldots \ldots \ldots$

B.20 2.245GHz, Transmitter $2 \ldots \ldots \ldots \ldots \ldots \ldots$

B.21 5.4GHz, Transmitter $2 \ldots \ldots \ldots \ldots \ldots$ 


\section{Introduction}

The pervasive application of wireless communications is well known. One such application is to an indoor factory environment. This environment creates challenges for reliable communications. One potential communication system that could be used to provide wireless communication in this environment is a Zigbee radio. Zigbee radios use about $2 \mathrm{MHz}$ of bandwidth in the $2.4 \mathrm{GHz}$ ISM band. To understand the performance of a Zigbee radio (or any other radio) in this environment the first task is to understand the propagation effects of such an environment. NIST has carried out a measurement campaign at various factory environments, including their own machine shop. We have used these measurements to develop channel models that are suitable for evaluating the performance of a Zigbee radio or any radios with bandwidth up to about $20 \mathrm{MHz}$ such as a WiFi based system. This report documents the results of processing the measurements to obtain channel models. Typical results for measurements made in the NIST machine shop for a $2.4 \mathrm{GHz}$ frequency are shown in the body of this document. Results for other frequencies and at other locations are shown in the appendicies.

\section{Measurements}

The channel measurement or sounding campaign was carried out by NIST at various factory or factory-like environments. For three days in April 2015, NIST researchers from Boulder, CO measured impulse response data in a working factory setting (a machine shop) in Gaithersburg, MD. The NIST researchers used a cart containing a mobile receiver that moved along a set path defined and measured the received signal from a transmitter located in the shop. The measurements of the channel was done by transmitting a $40 \mathrm{MHz}$ wideband signal using a pseudo-noise signal (m-sequence) that was mixed to a carrier frequency $(2.4 \mathrm{GHz}$ and $5 \mathrm{GHz}$ ). The receiver mixes the received signal to baseband and then samples the signal at an $80 \mathrm{MHz}$ rate. This system then allows for the calculation of the channel transfer function in a bandwidth of about $40 \mathrm{MHz}$. Since the Zigbee radios only use about $2 \mathrm{MHz}$ of bandwidth, this is more than adequate to obtain a channel impulse response that would be of use in analyzing the performance of Zigbee radios. It is also more than adequate for WiFi signals.

The measurements were made at NIST in Gaithersburg, Maryland at the machine shop. Figure 1 below shows the layout of the room. There are a number of machines in the room. A number of measurements were taken. Generally, the receiver was moved from the "Start" location through the room and ended up back at the start (also location 11 on map). Various check points with known locations (e.g. locations "Start", 1,..,11) were identified with particular acquisitions of received responses. In between these known locations for certain acquisitions it was assumed that the receiver moved at a constant speed. By knowing the coordinates of the different check points and the associated measurements, the location of the receiver for other measurements could be determined. As will be seen below, we believe that the location of the measurements was not as 


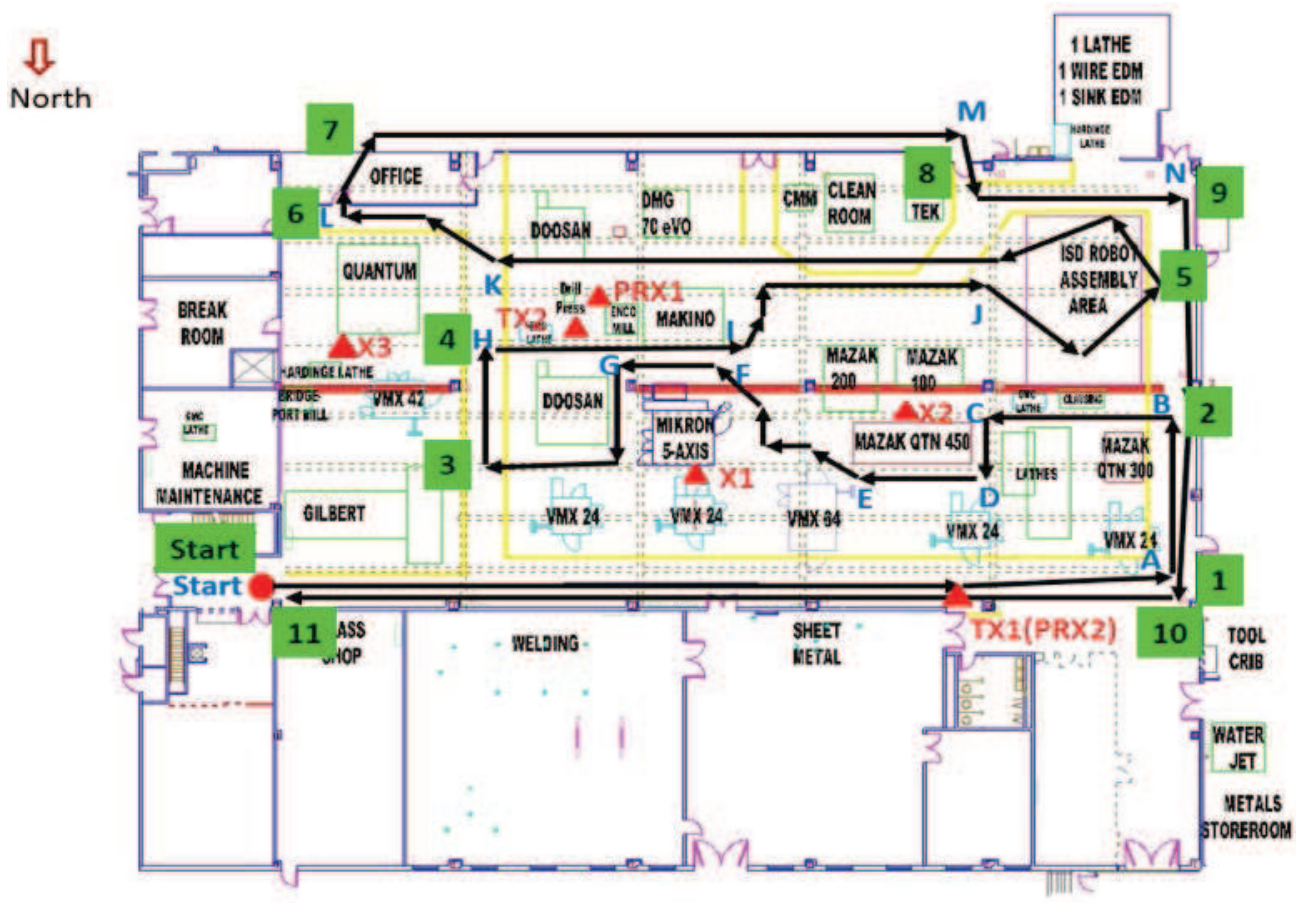

Figure 1: Layout of Room

accurate as needed to develop the most accurate channel models to show the path loss as a function of distance. It appears on some measurements that the received power reached a peak slightly earlier in acquisition number than the distance reached a minimum. So we believe the correspondence of the distance to the acquisition should be improved in the future. While the inaccuracies in location of the receiver for individual estimates will affect the model for propagation loss as a function of distance, it will not affect other channel estimates such as RMS delay spread, K factor, shadowing variance which generally are distance independent.

The basic setup of the channel sounding is illustrated in Figures 2 and 4. The transmitter and receiver have very accurate clocks that were initially synchronized. The transmitted signal was a m-sequence of length 2047. It was used as the input to a transmitter circuit.

The PN code is an m-sequence of length 2047 using shift register feedback connection as shown in Figure 3. This code generates the sequence $1010000000010000101110 \ldots$ of length 2047. The signal is generated by first mapping the m-sequence values, 0 and 1 , to +1 and -1 respectively and then repeating each chip four times at a sample rate of $80 \mathrm{M}$ samples/second. The duration of the signal is $T=8188 /\left(80 \times 10^{6}\right)=102.35 \mu \mathrm{s}$. Corresponding to each transmission there is a recording of the received signal after mixing down to baseband. The recorded signal is a complex signal corresponding to an IQ demodulation. 


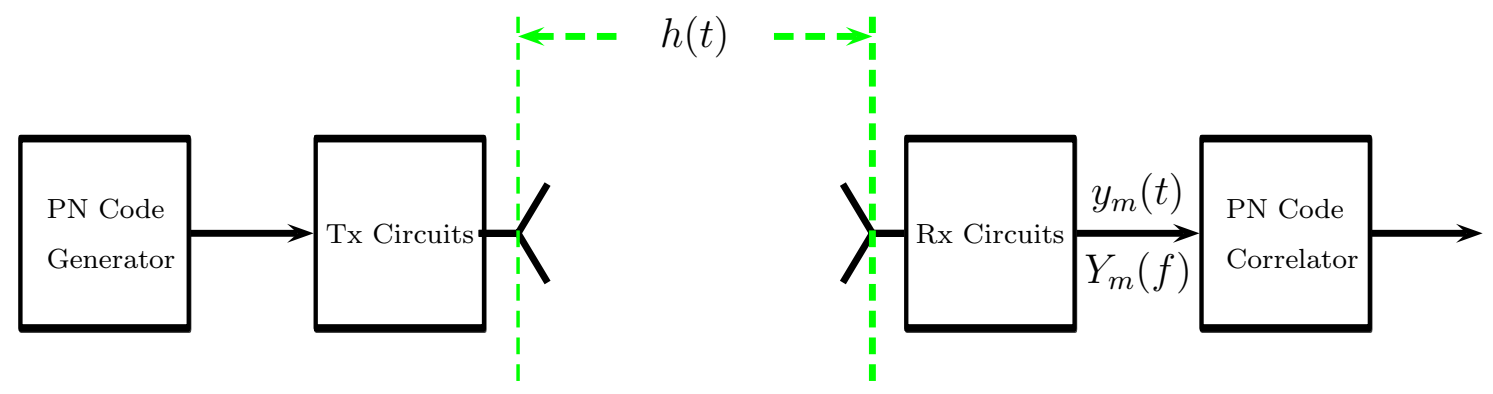

Figure 2: Single Input, single-output channel sounding system

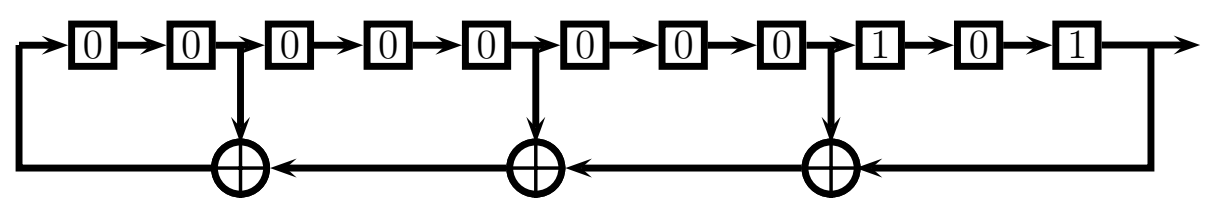

Figure 3: Generator for m-sequence

In order that the equipment not influence the calculation of the channel characteristic, a measurement was made with only an attenuator inserted between the transmitter and receiver (without the antennas) as shown in Fig. 4. To determine the channel

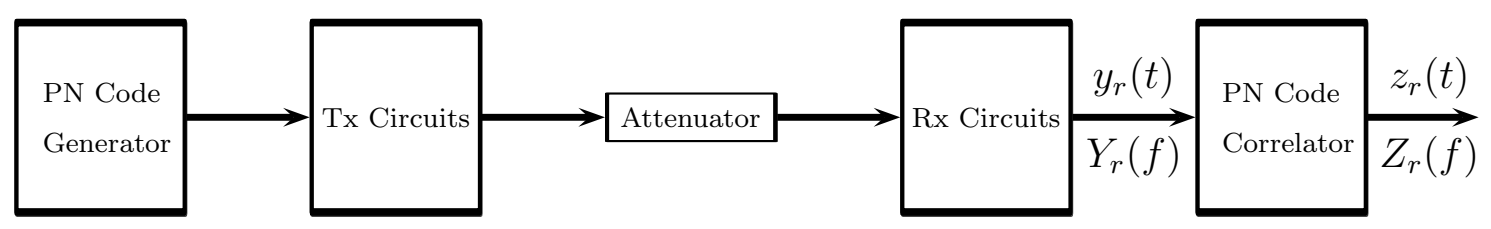

Figure 4: Single Input, single-output channel sounding system, reference measurement

characteristics (e.g. impulse response) we process the signal with a filter that is matched to the m-sequence generated signal at the transmitter. Because the signal is a 2047 chip m-sequence with 4 samples per chip there are 8188 samples of the received signal. The matched filter operates on complex (I and Q) samples of the receiver output. The magnitude of the normalized output of the filter matched to the m-sequence is shown in Fig. 5 where the normalization is such that the peak output value is 1 . A more detail 


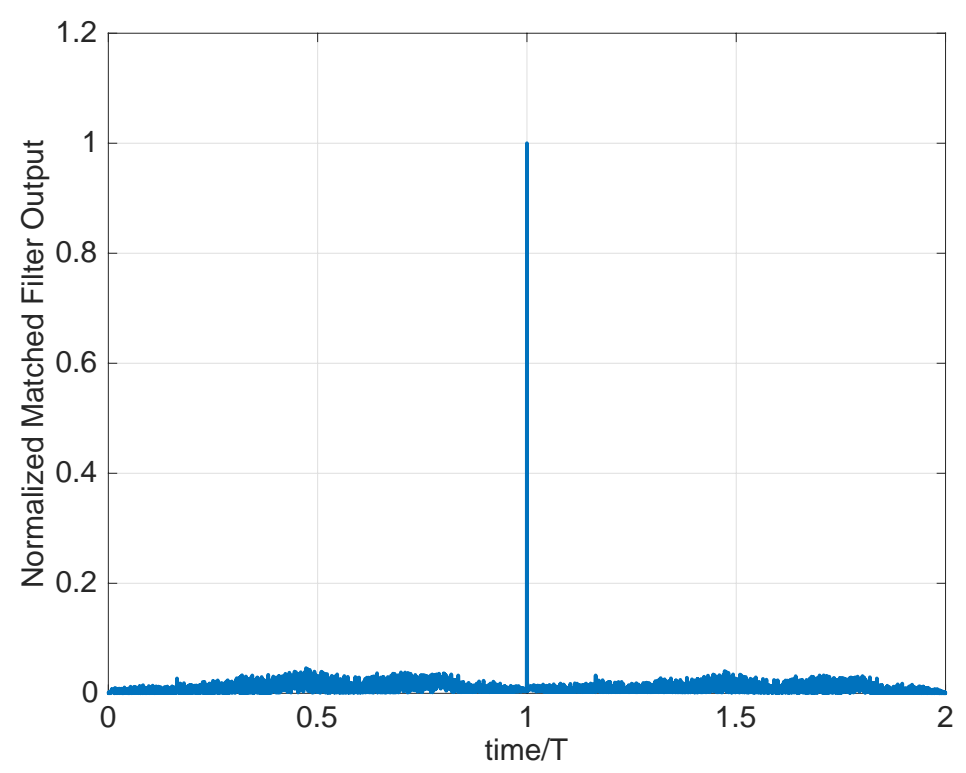

Figure 5: Output of Matched Filter for Reference System

examination of the matched filter output shown in Fig. 6 around the time where the signal reaches a peak shows the typical triangular shape of the autocorrelation of the m-sequence signal.

There are several parts of our effort to characterize the channel. The first part is to determine the received power as a function of distance and to generate an appropriate model. In this part of our characterization it is only the received power that is of importance as opposed to the actual channel impulse response. To determine the attenuation we measured the power in the measured signal and then compared to the power in the reference signal. Factoring in the attenuation of the reference signal we can obtain the received power of the channel (including the antennas). To determine the received power as a function of distance we calculate the power received in the IQ samples and compare that to the power received in the reference system taking into account the attenuator. Let

$$
\begin{aligned}
P_{r} & =\sum_{i=0}^{N-1}\left|y_{r}(i)\right|^{2} \\
P_{m} & =\sum_{i=0}^{N-1}\left|y_{m}(i)\right|^{2} .
\end{aligned}
$$

Then the received power is determined as

$$
P L(d)=\frac{P_{m} A}{P_{r}}
$$




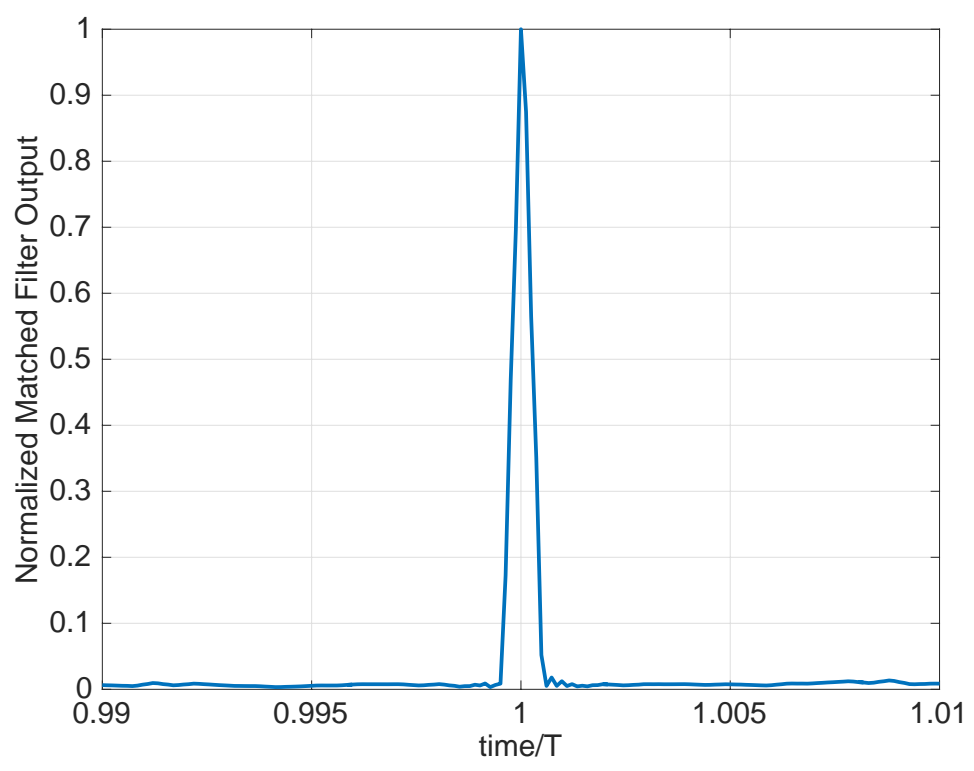

Figure 6: Output of Matched Filter for Reference System

where $A$ is the value of the attenuation in the reference system. The value used for attenuation was $-49.8 \mathrm{~dB}$ at a frequency of $2.4 \mathrm{GHz}$. The attenuation was slightly larger at $5 \mathrm{GHz}$ frequencies. By generating a received power at each distance the received power can be determined as a function of distance. The received power as the receiver moved through various places is shown in Fig. 7. The number of measurements for a particular polarization and frequency was on the order of 10,000. Each of these is called an acquisition. The received power as a function of acquisition number and the distance as a function of acquisition number is shown in Figure 7 . One can clearly see that the distance achieves a minimum at acquisition about 1300 while the peak in the received power is closer to acquisition 1000. While it is possible to receive smaller power at closer distances, it is unlikely. Typically, as shown below, the model for the received power is an inverse power law model where the power received is inversely proportional to the distance raised to a power: $P_{r}=k / d^{\alpha}$ for $d>d_{0}$. Our model, however, is a piecewise linear version in that over some initial range of distances there is one value for $\alpha$ and then at larger distances there is a second value for $\alpha$.

$$
\begin{gathered}
\Gamma=\frac{P_{r}}{P_{t}}= \begin{cases}k_{1} / d^{\alpha_{1}}, & d<\beta \\
k_{2} / d^{\alpha_{2}}, & d>\beta .\end{cases} \\
\Gamma[\mathrm{dB}]= \begin{cases}k_{1}[\mathrm{~dB}]-\alpha_{1} 10 \log _{10}(d), & d<\beta \\
k_{2}[\mathrm{~dB}]-\alpha_{2} 10 \log _{10}(d), & d>\beta .\end{cases}
\end{gathered}
$$

In these equations $P_{t}$ is the transmitted power, $P_{r}$ is the received power. The channel model constants $k_{1}, k_{2}$ and $\alpha_{1}, \alpha_{2}$ are to be determined from the measurements. Some of 


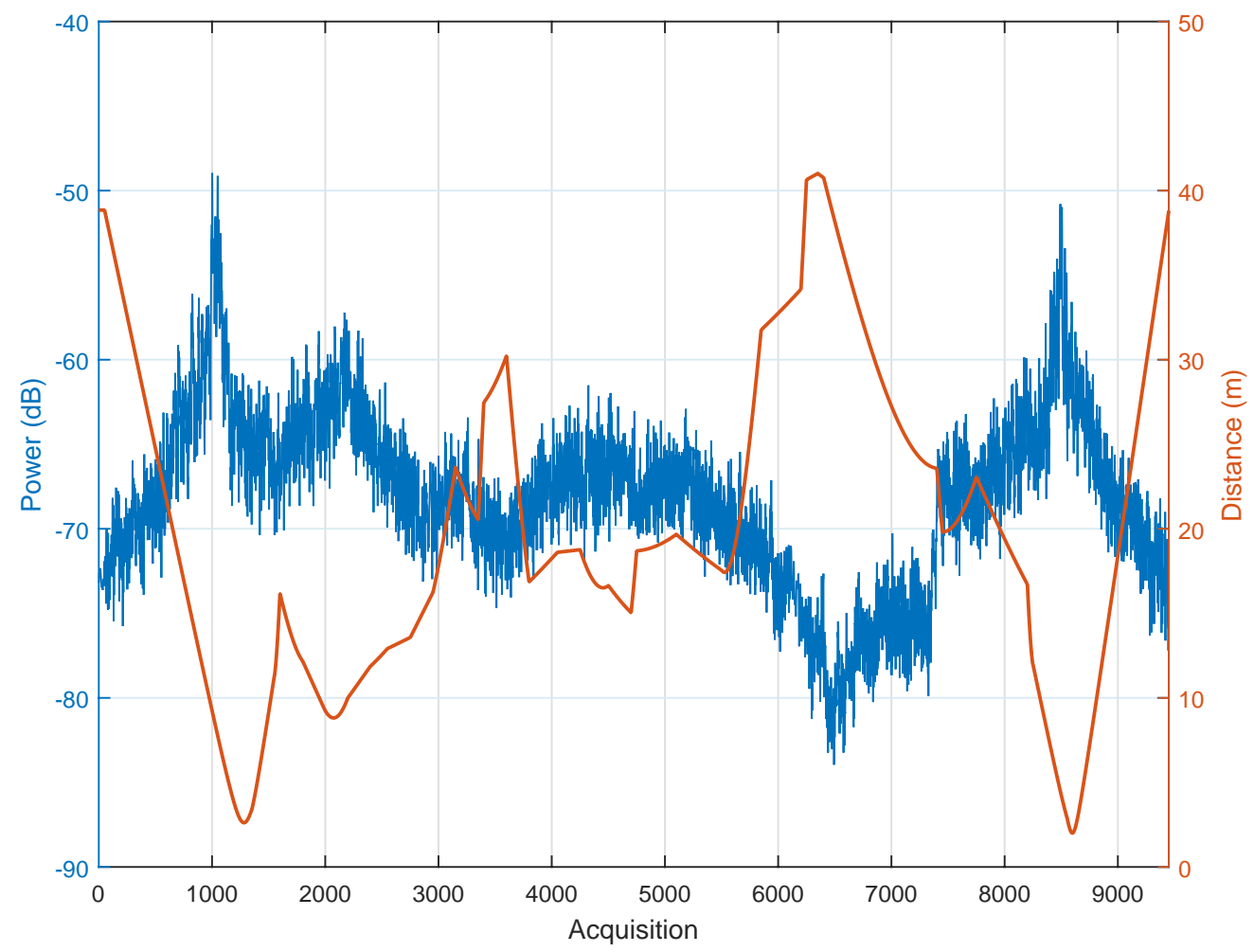

Figure 7: Received Power vs. Acquisition and Distance vs. Acquisition, Cross Polarization, 2.4GHz, Transmitter Location 1.

the results for this model are shown below based on the Gaithersburg measurements. The received wower is split it to two possible sets; line-of-sight set and nonline-of-sight set. Figs. 8, 9, and 10 show the received power for a $2.4 \mathrm{GHz}$ system with vertical polarized antennas.

The parameters of the model were found based on finding the smallest mean squared error between the model and the measurements. The parameters of the model are shown in the following table for this particular configuration (Cross Polarization, 2.4GHz, Transmitter Location 1. Note that the mean square error of the measurements is also the variance corresponding to a log-normal distribution of the received power.

\begin{tabular}{c|c|c|c|c|c|c|c|c}
\hline Type & Frequency & Polarization & $\alpha_{1}$ & $\alpha_{2}$ & $\beta(\mathrm{m})$ & $k_{1}(\mathrm{~dB})$ & $k_{2}(\mathrm{~dB})$ & MSE \\
\hline \hline Overall & $2.245 \mathrm{GHz}$ & CrossPol & -0.47776 & 2.166 & 8.4098 & -64.9408 & -40.4919 & 9.3129 \\
\hline LOS & $2.245 \mathrm{GHz}$ & CrossPol & -0.48848 & 1.6744 & 7.8537 & -64.8572 & -45.4973 & 9.5928 \\
\hline NLOS & $2.245 \mathrm{GHz}$ & CrossPol & 1.4082 & 3.3221 & 20.3506 & -49.7419 & -24.6974 & 8.0049 \\
\hline
\end{tabular}

Table 1: Parameters for Cross Polarization, 2.4GHz, T1 


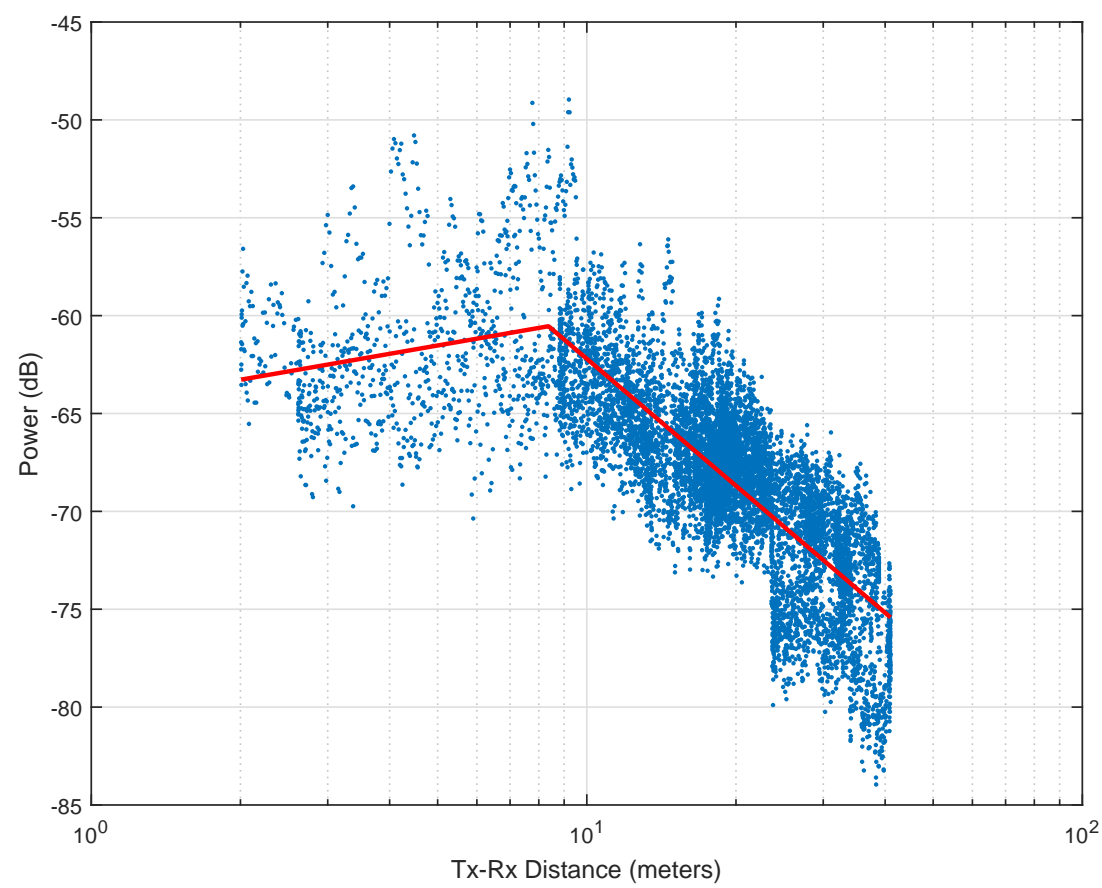

Figure 8: Overall Received Power vs. Distance, Cross Polarization, 2.4GHz, Transmitter Location 1. 


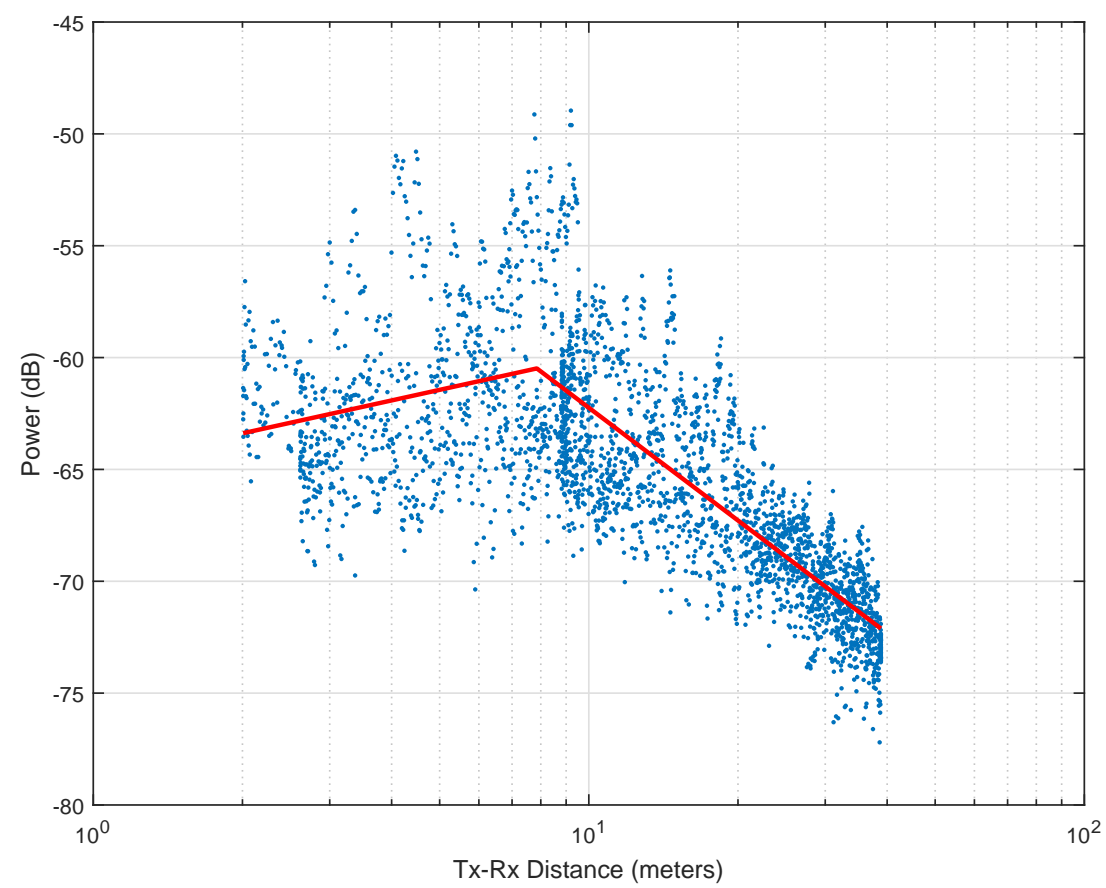

Figure 9: Line of Sight Received Power vs. Distance, Cross Polarization, 2.4GHz, Transmitter Location 1. 


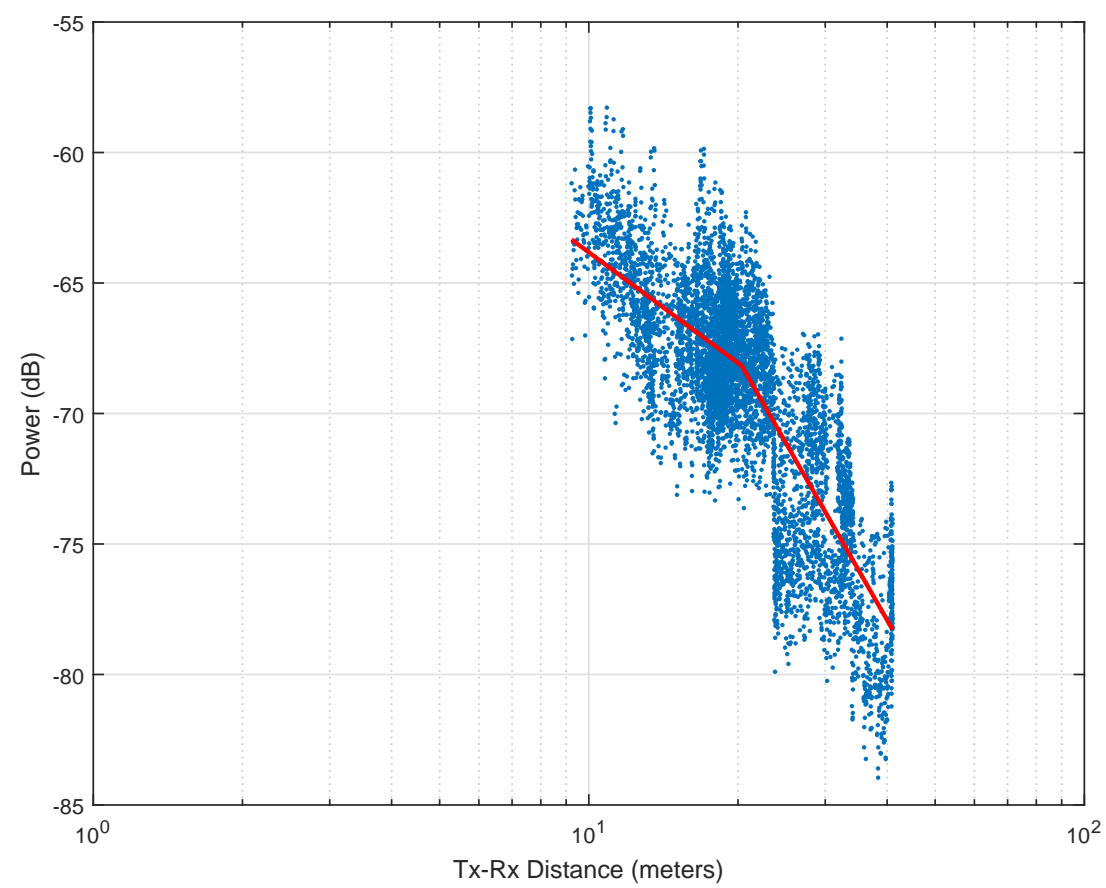

Figure 10: Non Line of Sight Received Power vs. Distance, Cross Polarization, 2.4GHz, Transmitter Location 1. 


\section{Received Power Channel Models}

There have been various measurements of propagation at $2.4 \mathrm{GHz}$ in industrial settings. In this section various models are described for the propagation of the signal in terms of just the received power. As a baseline the free space received power is the ratio of received power to transmit power, $\Gamma=P_{r} / P_{t}$. In free space the received power is

$$
\Gamma[\mathrm{dB}]=-20 \log _{10}(f[\mathrm{MHz}])-20 \log _{10}(d)+27.5
$$

where $f[\mathrm{MHz}]$ is the frequency in MHz. For $f=2450 \mathrm{MHz}$ this becomes

$$
\Gamma[\mathrm{dB}]=-40.28-20 \log _{10}(d)
$$

A report [1] has the following received power model. This model has a slope of 40dB per decade of distance decrease in power, or a received power exponent of 4 .

$$
\Gamma[\mathrm{dB}]=-50.3-40 \log _{10}(d)
$$

Another report [2] has the following received power models. We will call these model 2a and model $2 \mathrm{~b}$. These models have a slope of $18 \mathrm{~dB}$ per decade and $13.7 \mathrm{~dB}$ per decade of distance decrease in power, or a received power exponent of 1.8 and 1.4.

$$
\begin{aligned}
& \Gamma[\mathrm{dB}]=-59.96-18.4 \log _{10}(d) \\
& \Gamma[\mathrm{dB}]=-53.86-13.7 \log _{10}(d)
\end{aligned}
$$

A paper [3] has a received power model for indoor radio channels.

$$
\Gamma[\mathrm{dB}]=-54.5-16.4 \log _{10}(d)
$$

Another paper [4] has a received power model for indoor radio channels.

$$
\Gamma[\mathrm{dB}]=-43.1-33.3 \log _{10}(d)
$$

Yet another paper [5] has several received power models for indoor radio channels. These are single slope models. They propose models where $P L\left(d_{0}\right)$ is determined based on one of two possible methods. In both methods the distance is fixed at $15 \mathrm{~m}$. In one method the value of $P L\left(d_{0}\right)$ is chosen to minimize the mean square error of the fit. In a second method the received power at distance $d_{0}$ is chosen to match the free space received power at that distance.

$$
\Gamma[\mathrm{dB}]=-P L\left(d_{0}\right)-n \log _{10}(d) ;
$$

For 2.4 GHS frequencies they have the following parameters for the best match choice for $P L\left(d_{0}\right)$. 


\begin{tabular}{|c|c|c|}
\hline Conditions & $P L\left(d_{0}\right)$ & $\mathrm{n}$ \\
\hline \hline LOS & 67.43 & 1.72 \\
\hline NLOS (light clutter) & 72.71 & 1.52 \\
\hline NLOS (heavy clutter) & 80.48 & 1.69 \\
\hline
\end{tabular}

Finally another paper [6] has a received power (or attenuation) measurement. The measurements show

$$
\Gamma[\mathrm{dB}]=\left\{\begin{array}{cc}
-56 & d=6 \mathrm{~m} \\
-61 & d=11 \mathrm{~m} \\
-62.5 & d=15 \mathrm{~m} \\
-64.8 & d=19 \mathrm{~m} .
\end{array}\right.
$$

In Fig. 11 we compare plot the received power for these models for versus distance. All models have a simple linear representation of received power in $\mathrm{dB}$ versus log distance, we have a piece-wise linear model with two different slopes. We will compare our models with this baseline model. We will not repeat in each case where the other models are from. Our model will be the plot with the largest linewidth.

We have determine the slopes and the break point between the two slopes in order to minimize the mean square error between the measurements and the model. The parameters of the model are in the tables above along with the resulting mean square error. Note that the mean square error is essentially the variance of the log-normal shadowing of the system. In the appendices we compare our model with these.

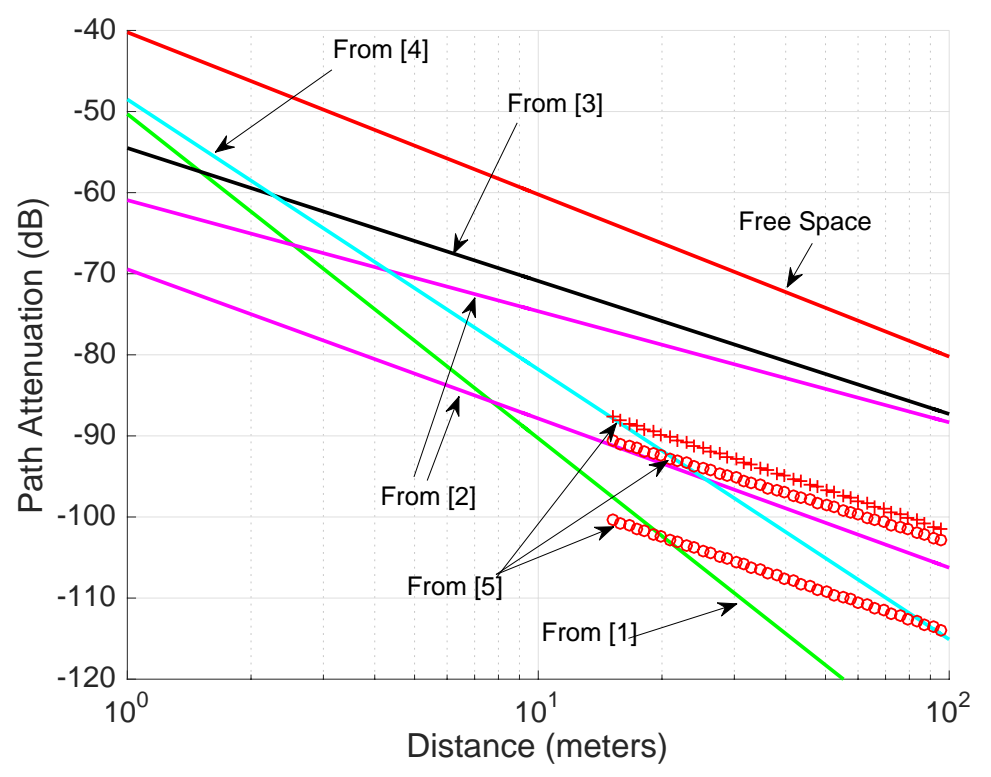

Figure 11: Overall received power $(2.4 \mathrm{GHz})$ 


\section{Impulse Response}

The measurement results allow us to estimate the channel impulse response. As a first estimate consider the output of the reference system after performing a matched filter and the corresponding output of the measurement system.

$$
\begin{aligned}
z_{r}(t) & =y_{r}(t) * h_{M F}(t) \\
& =\left[s_{T}(t) * h_{T}(t) * h_{R}(t) * h_{M F}(t)\right] A .
\end{aligned}
$$

where $s_{T}(t)$ is the signal generated by the m-sequence generator, $h_{T}(t)$ and $h_{R}(t)$ are the impulse responses for the transmitter and receiver circuitry and $A$ is the attenuation value. The corresponding output for the measurement system is

$$
\begin{aligned}
z_{r}(t) & =y_{m}(t) * h_{M F}(t) \\
& =\left[s_{T}(t) * h_{T}(t) * h(t) * h_{R} X(t) * h_{M F}(t)\right] \\
& =\left[s_{T}(t) * h_{T}(t) * h_{R} X(t) * h_{M F}(t)\right] * h(t) \\
& =\frac{z_{r}(t) * h(t)}{A} .
\end{aligned}
$$

As seen above, the output of the matched filter for the reference systems, $z_{r}(t)$ is nearly an ideal impulse function. As a result, the channel $h(t)$ can be estimated simply as $\hat{h}(t) \approx z_{r}(t) A$. The result of this estimate is shown in Fig. 12 for one such measurement. Generally the amount of power decreases as the delay increases due to the larger propagation loss of a path that takes longer to reach the receiver.

The magnitude of the impulse responses for a set of 10500 acquisitions is shown in Fig. 13. Often the channel is modeled as a series of impulses of the form

$$
h(t)=\sum_{i} \alpha_{i} \delta\left(t-\tau_{i}\right)
$$

where $\alpha_{i}$ is a complex path gain at delay $\tau_{i}$. For this channel model the result of the measurement would be

$$
\begin{aligned}
z_{m}(t) & =\frac{1}{A} z_{r}(t) * h(t) \\
& =\frac{1}{A} z_{r}(t) * \sum_{i} \alpha_{i} \delta\left(t-\tau_{i}\right) \\
& =\frac{1}{A} \sum_{i} \alpha_{i} z_{r}\left(t-\tau_{i}\right) .
\end{aligned}
$$

Our goal is to determine the values for $\alpha_{i}$ and $\tau_{i}$. Since the function $z_{r}(t)$ is known the approximation used above is that $h(t)$ is just a normalized version of $z_{m}(t)$. However, 


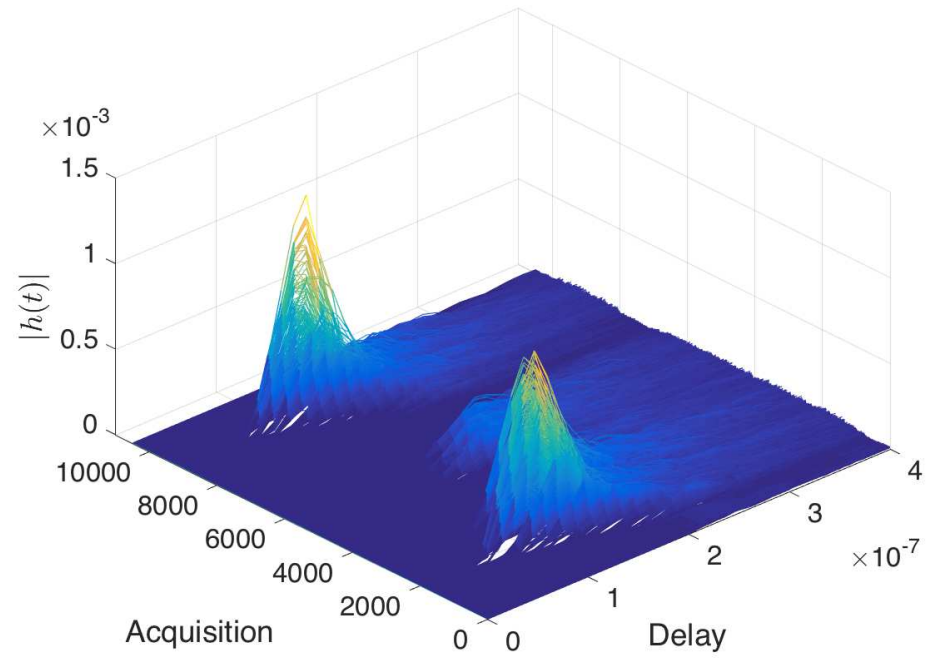

Figure 12: Impulse Response Estimate

we can also calculate the $\alpha_{i}$ by using the known value of $z_{r}(t)$. In particular we can determine the largest value of $\alpha_{i}$ by looking at the largest value of the measurement and the associated delay and associating that output value with of $\alpha_{i}$. With that determined we can subtract off the effect of the largest $\alpha_{i}$, namely $\alpha_{i} z_{r}\left(t-\tau_{i}\right)$ and continue the process to find the second largest value of $\alpha_{i}$ and the associated delay. This is generally known as the CLEAN algorithm [7]. Below in Fig. 14 we show the impulse response as calculated above for one of the acquisitions and then the result of applying the CLEAN algorithm. The error is the left over signal after 250 iterations of the CLEAN algorithm where in each iteration the largest magnitude signal is accounted for by a particular delay and coefficient of the channel. This is shown in $\mathrm{dB}$ on the left and not in $\mathrm{dB}$ on the right. Note that a particular delay could correspond to several iterations that have the largest magnitude residual error signal. In this case it is the (complex) sum of these coefficients that determine the final coefficient at that delay. 


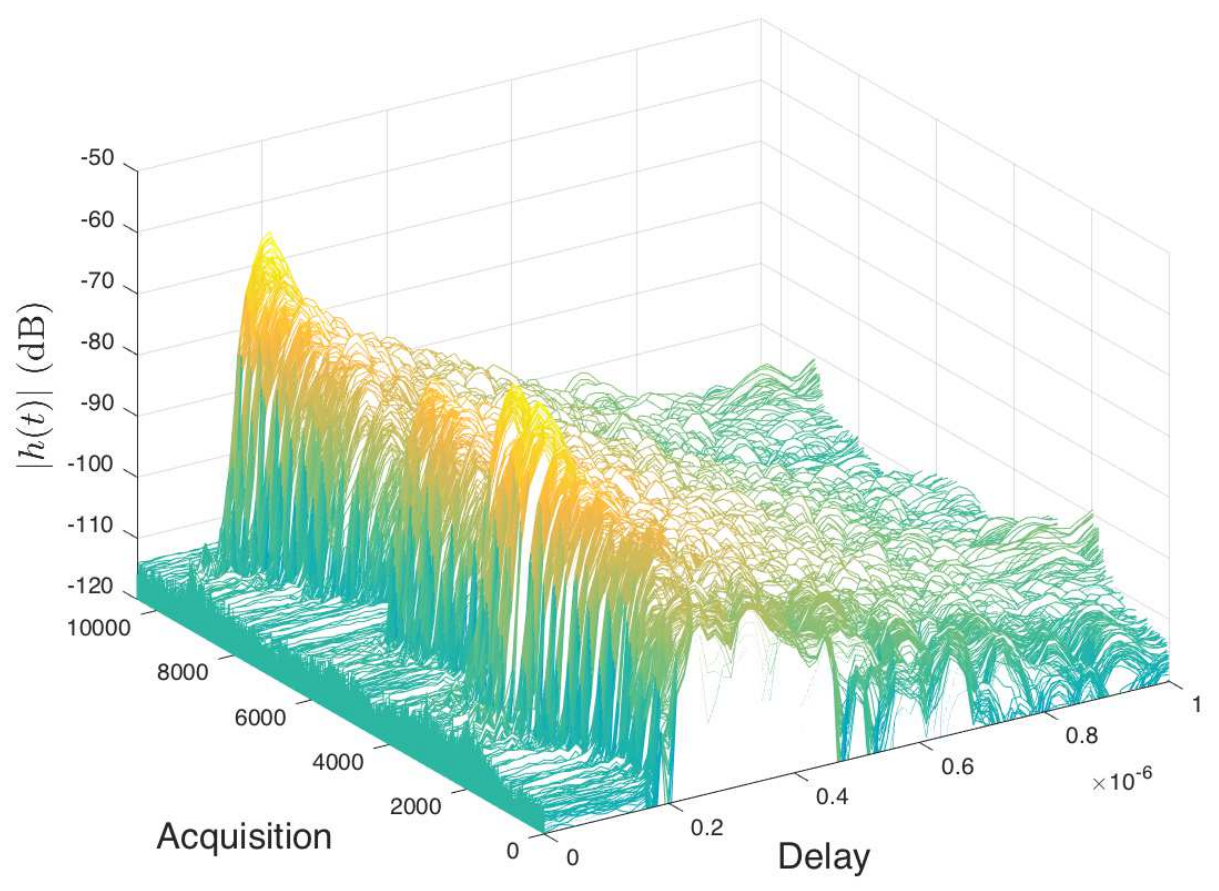

Figure 13: Impulse Responses
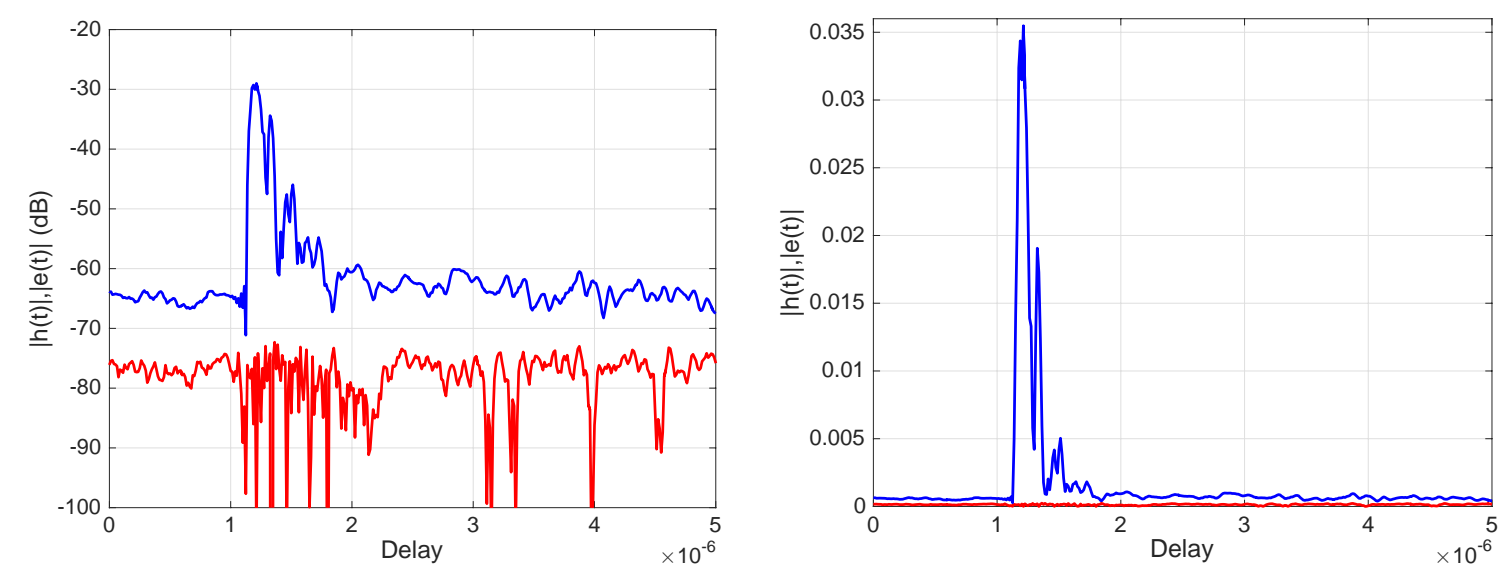

Figure 14: Impulse response (blue) and error signal (red)

\section{Zigbee Performance}

In this section we evaluate the error probability for a Zigbee communication system under various propagation conditions. As is known, the 802.15.4 standard specifies how signals are to be transmitted but not how signals are to be received. Thus in order to 
simulate the performance a receiver structure must be decided on. We consider both a coherent receiver and a noncoherent receiver. A coherent receiver will likely have better performance in terms of error probability at a given received signal-to-noise ratio but require more power to operate the receiver. A noncoherent receiver will require higher received signal-to-noise ratio in order to achieve the same error probability as the coherent receiver but will consume less power to operate the receiver. There are different variations on these types of receivers that have different performance. In Fig. 16 the error probability of a IEEE 802.15.4 system with a coherent receiver and a noncoherent receiver for a packet of length 127 bytes is shown for an AWGN channel. As can be seen the coherent receiver is less than $2 \mathrm{~dB}$ better than the noncoherent receiver. One reason for such a small gap is that the modulation used in Zigbee is a version of 16-ary orthogonal modulation. As is known, orthogonal modulation has asymptotically (for large number of signals) the same performance for coherent reception and noncoherent reception. Here the signal-to-noise ratio $\left(E_{b} / N_{0}\right)$ is the average received energy per information bit to noise power spectral density.

In indoor and outdoor applications, radio systems need to have a good performance which means a reasonable amount of information loss. As any other radio system, in order to evaluate Zigbee performance, we started with simulating Zigbee in an additive white Gaussian noise (AWGN) channel as well as Ricean fading channels.. In IEEE802.15.4, at the beginning of PPDU of each packet, there is a 4 bytes-long preamble which consists of 32 zero bits for all packets. We are using these 32 zero bits to find the start of each packet using a matched-filter which is matched to each symbol (4 zeros) of the preamble. After creating I and Q baseband signals, they are combined into a complex transmitted signal.

The transmitted signal is passed through a complex AWGN channel and the output of the channel is fed into the Zigbee receiver. As the first block of any radio-system receiver, a synchronization block is designed to find the start of each packet. Since there is a fixed pattern in preamble part of each packet, the receiver uses a matched-filter to locate the separating flag between any two consecutive packets. After finding start of packet, it is possible to pass preamble and demodulate the length of payload-byte of PPDU. Knowing the packet start and the packet length, then the next step is to demodulate the payload which carries the information bits. The demodulation is 16-orthogonal demodulation and is used to detect payload of each packet. The magnitudes only of the 16 demodulator outputs are used to make a decision about the data for the non-coherent receiver. To do coherent demodulation, the real part of outputs of inner products are considered and the maximum is selected. Both coherent and non-coherent receiver have been simulated and their performances in terms of Packet Error Rate (PER) are compared above in Fig. 16.

The indoor channel environment are not always well modeled by an AWGN channel. Multipath propagation and obstacle reflections can have a significant impact on system performance. In order to model the multipath propagation, which is serious factor in indoor-communication applications, a Rician fading channel has been simulated. Rician fading is a stochastic model for the radio propagation when the signal arrives at the 
receiver by several different paths. Rician fading can nicely model the environment specially, when one path, which is usually line of sight path, is much stronger than others. This appears to be the case for some of the indoor industrial channel for Zigbee since the bandwidth is relatively small $(2 \mathrm{MHz})$ compared to the bandwidth for WiFi $(20 \mathrm{MHz})$. Our simulation models the amplitude gain using a Rician distribution. Rayleigh fading is used to model the multipath propagation when there is no line of sight. A Rician model with different ratio of direct line-of-sight power versus diffuse power, known as the $\mathrm{K}$ factor has been used in our simulation. The packet error rate for a packet of length 127 bytes (the longest possible packet length for Zigbee) is shown in Fig. 15. Zigbee performance to appear in Rayleigh channel and also the improvement in the performance in term of PER curves with increasing Rician $\mathrm{K}$ factor. As it is evident in the Figure 15, the PER converges to AWGN Packet Error Rate curve as the K factor gets large. Figure 16 shows the error probability for two different receivers when the block length is 127 bytes. One receiver is a coherent receiver while the other receiver is noncoherent. Figure 17 shows the error probability for coherent receivers when the block length is 127 bytes and the K-factor varies.

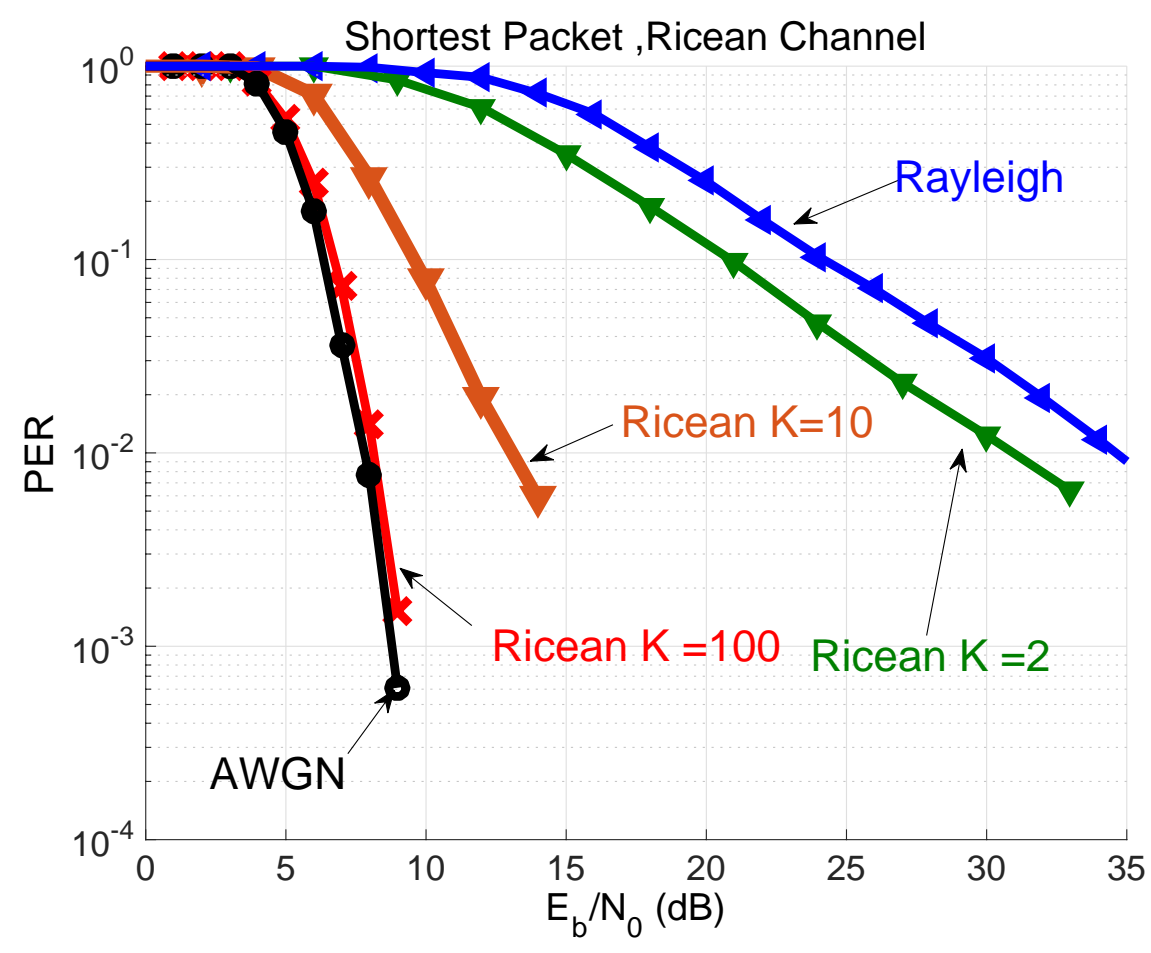

Figure 15: Packet Error Probability 


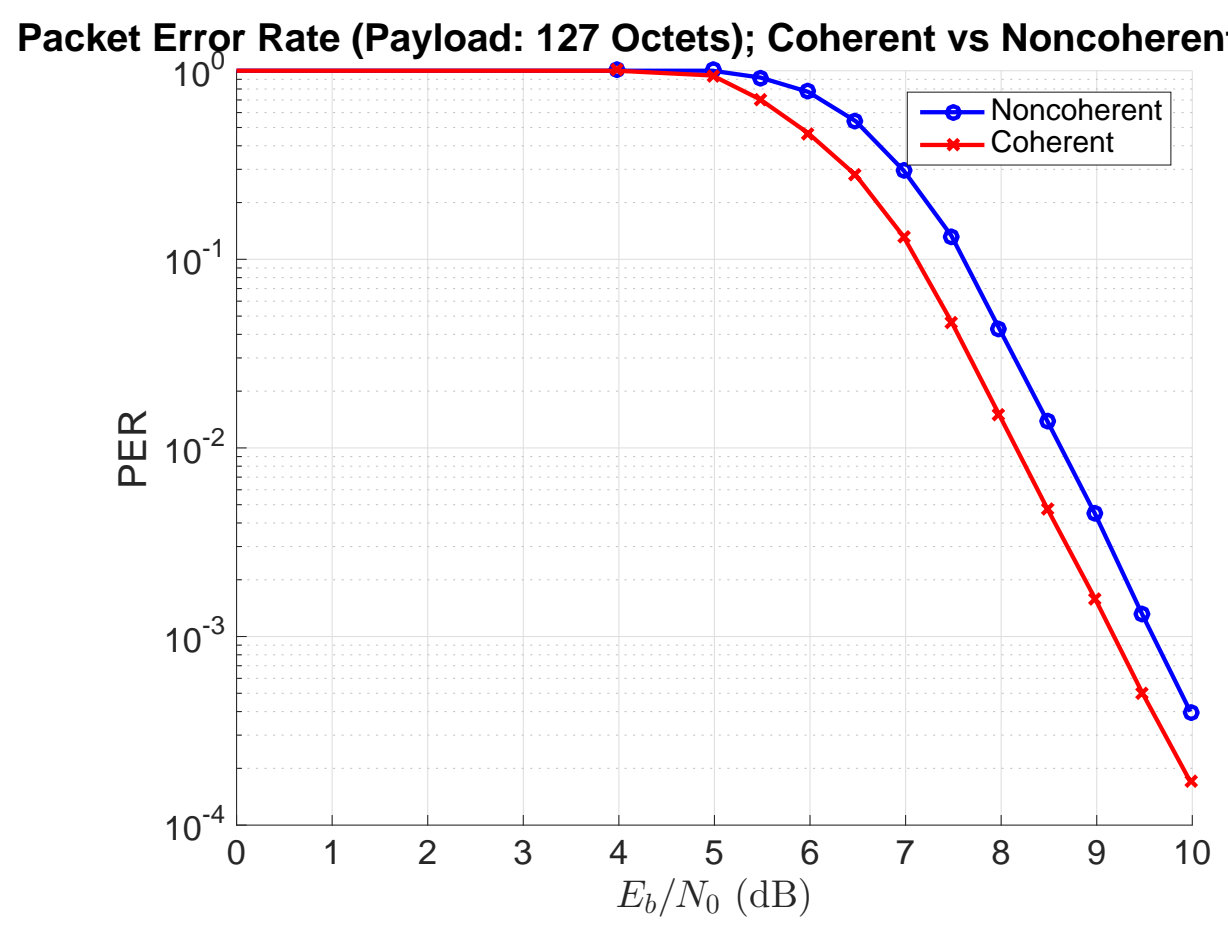

Figure 16: Packet Error Probability Block Length 127 Bytes: Coherent vs. Noncoherent, AWGN

\section{Conclusion}

In this report we have used measurements to obtain models for indoor industrial environment channels. Our models are piece-wise linear relations between the received power (in $\mathrm{dB}$ ) and the log of the distance. Perhaps the most useful part of the propagation model occurs after the breakpoint in the piece-wise linear model where the power received becomes small. The received power at short distances is larger than other models while at higher distances the received power is less than most other models. At distances smaller than the break point in the piece-wise linear model the received power is going to be quite large and the exact value of the received power is probably not important as the system will have more than adequate power to decode a packet correctly. We have used to CLEAN algorithm to determine the multipath channel characteristics. The multipath delay spread is generally less that $0.5 \mu \mathrm{s}$ and is comparable to the inverse bandwidth of a Zigbee system. That is, most of the multipath components will be within a single chip duration of a Zigbee signal. We have used the measurements to evaluate the shadowing parameter for this environment and our results show a log normal shadowing of between 7 and $12 \mathrm{~dB}$. A Zigbee radio system with different receivers has been simulated and the performance in different channel environments has been determined. While there is a small difference between coherent and noncoherent receivers (e.g. about $2 \mathrm{~dB}$ ), there is a large gap between AWGN performance and Rayleigh faded performance. This is to be 
Packet Error Rate (Payload: 127 Octets); Coherent Receiver AWGN vs Ricean and Rayleigh

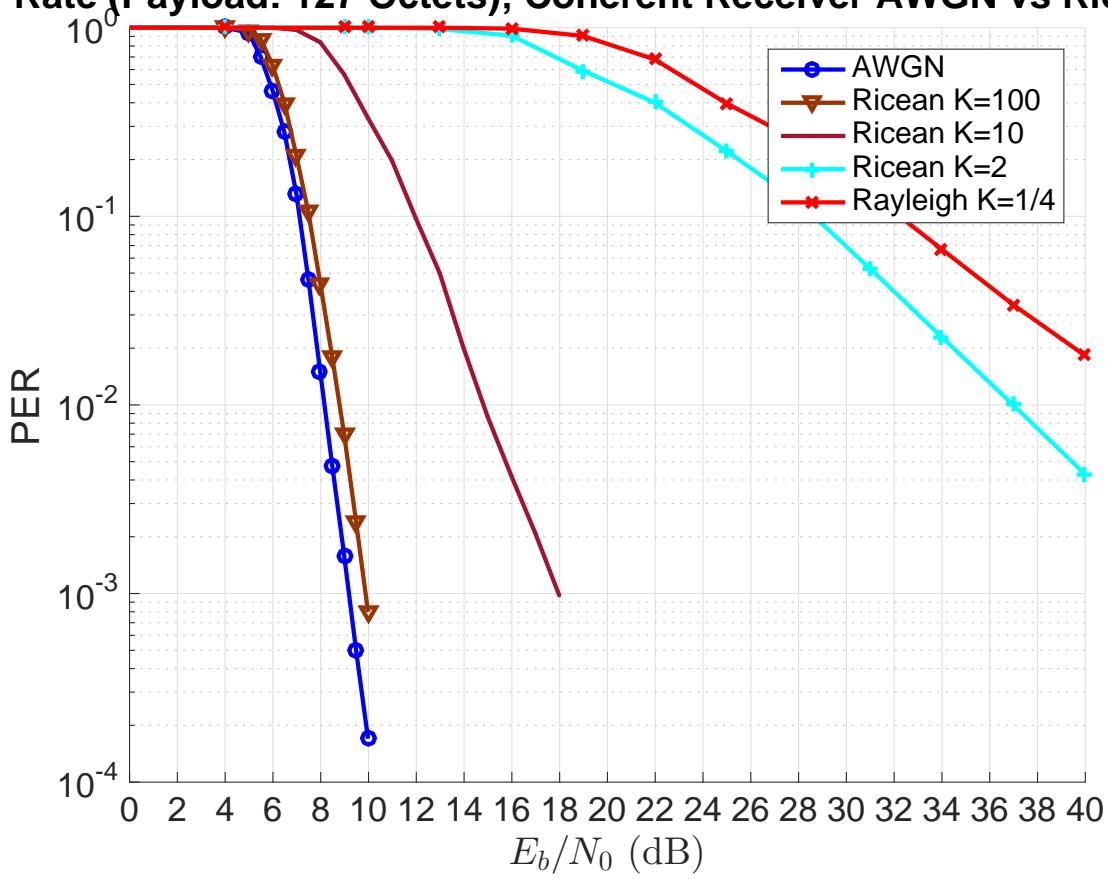

Figure 17: Packet Error Probability Block Length 127 Bytes: Coherent Ricean, Rayleigh

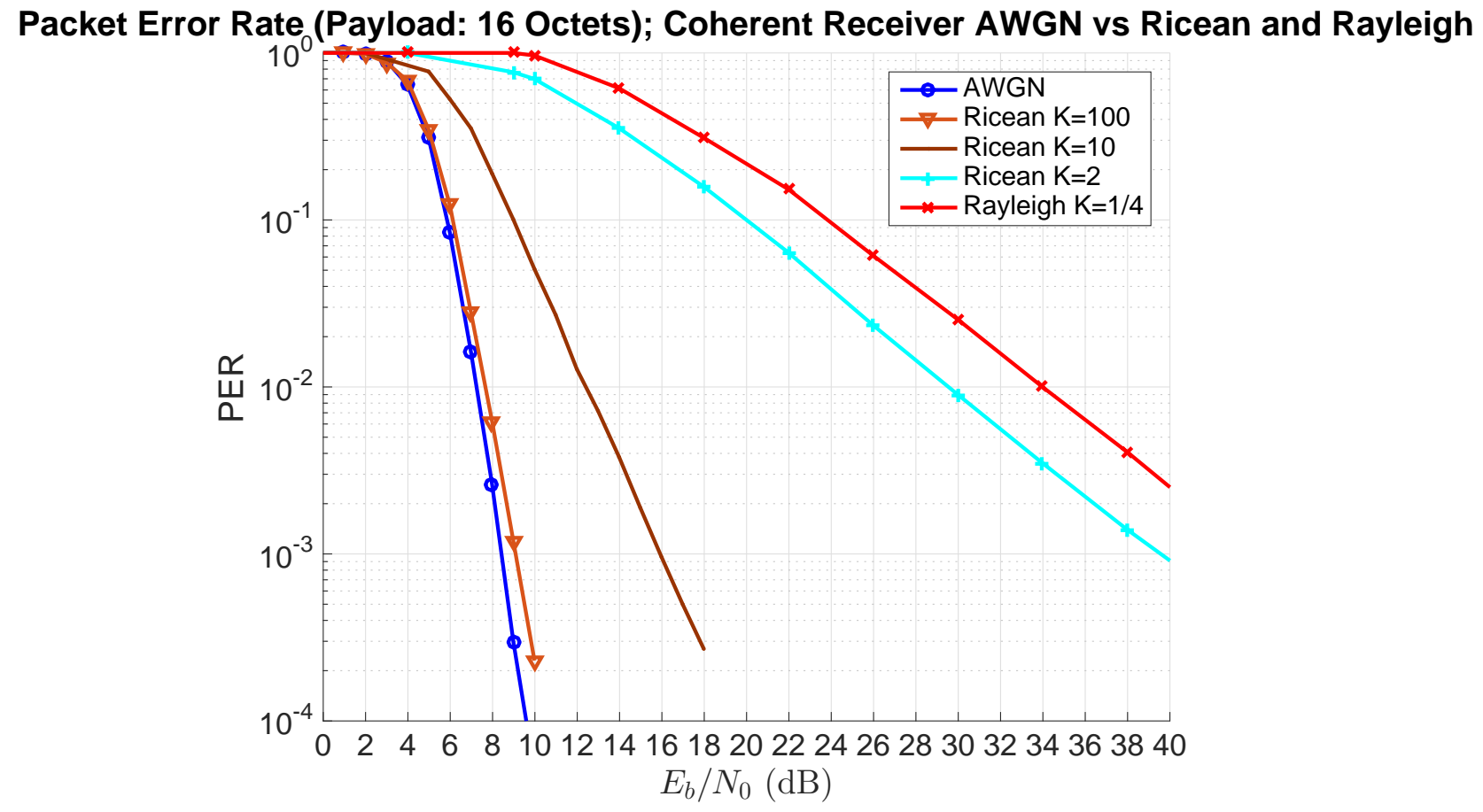

Figure 18: Packet Error Probability Block Length 16 Bytes: Coherent Ricean, Rayleigh 
expected since the Zigbee signals do not employ error-correcting codes or wide enough bandwidth so that the fading is mitigated. 


\section{References}

[1] S. Wloczysiak, "Extending $2.4 \mathrm{GHz}$ ZigBee short range radio performance with Skyworks front-end modules," Microwave Journal, pp. 1-10, August 2009.

[2] H. Li, L. Zhao, M. J. Darr, and P. Ling, "Modeling wireless signal transmission performance path loss for ZigBee communication protocol in residential houses," 2009 ASABE Annual International Meeting, 2009.

[3] C. Monti, A. Saitto, and D. Valletta, "Indoor radio channel models for ieee 802.15. 4 technology," Proceedings of EURASIP Workshop on RFID, 2008.

[4] G. J. Janssen, P. Stigter, R. Prasad, et al., "Wideband indoor channel measurements and BER analysis of frequency selective multipath channels at 2.4, 4.75, and 11.5 GHz," Communications, IEEE Transactions on, vol. 44, no. 10, pp. 1272-1288, 1996.

[5] E. Tanghe, W. Joseph, L. Verloock, L. Martens, H. Capoen, K. V. Herwegen, and W. Vantomme, "The industrial indoor channel: large-scale and temporal fading at 900, 2400, and $5200 \mathrm{MHz}$, "IEEE Transactions on Wireless Communications,, vol. 7, no. 7 , pp. 2740-2751, 2008.

[6] J. Ferrer-Coll, P. Ängskog, J. Chilo, and P. Stenumgaard, "Characterisation of highly absorbent and highly reflective radio wave propagation environments in industrial applications," IET Communications, vol. 6, no. 15, pp. 2404-2412, 2012.

[7] Q. Spencer, M. Rice, B. Jeffs, and M. Jensen, "A statistical model for angle of arrival in indoor multipath propagation," IEEE 47th Vehicular Technology Conference, vol. 3, pp. 1415-1419 vol.3, May 1997. 


\section{A Received Power Data: Gaithersburg}

In this appendix we present the rest of the data regarding received power for various measurements in Gaithersburg at the machine shop of NIST. Below we show the power received first as a function of acquisition number and simultaneously on the same plot show the distance as a function of acquisition number. Note that the line-of-sight and non line-of-sight were chosen manually. 


\section{A.1 Cross Polarization, 2.245GHz, Transmitter 1}

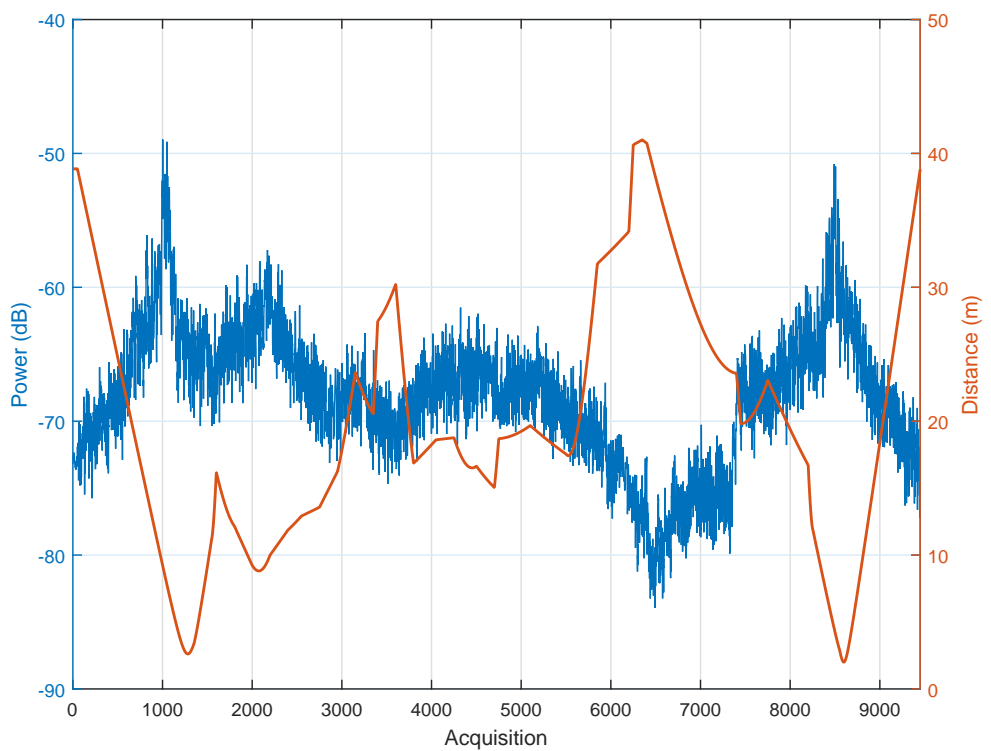

Figure 19: Overall Received Power vs. Acquisition Number and Distance vs. Acquisition Number: Cross Polarization, 2.245GHz, T1.

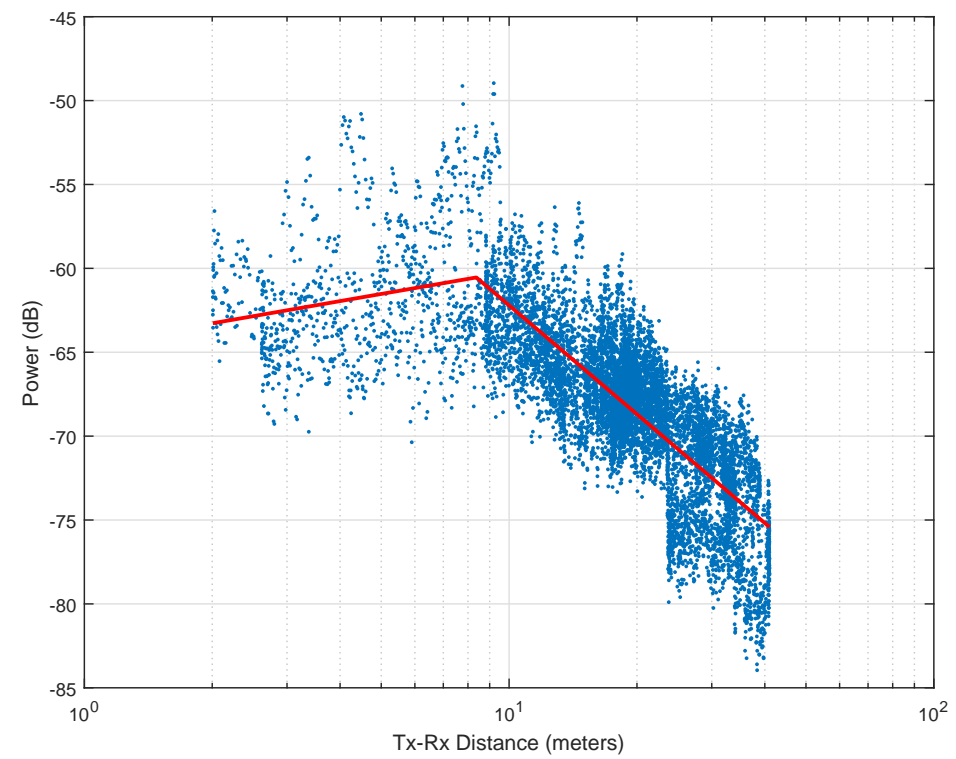

Figure 20: Overall Received Power vs. Distance: Cross Polarization, 2.245GHz, T1. 


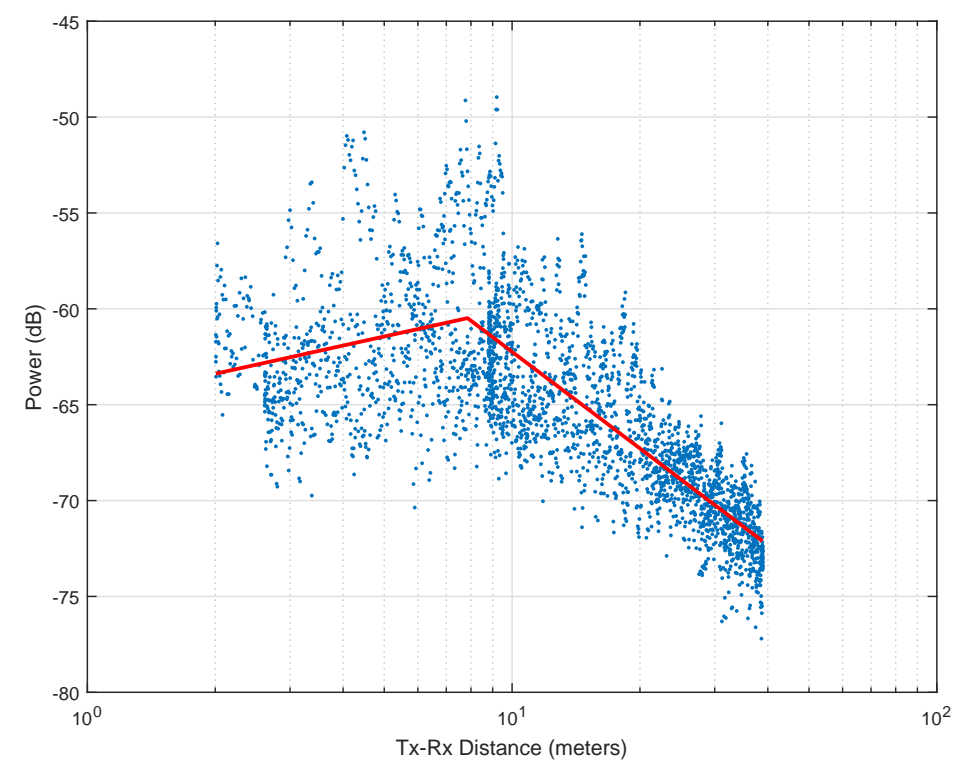

Figure 21: Line of Sight Received Power vs. Distance: Cross Polarization, 2.245GHz, $\mathrm{T} 1$.

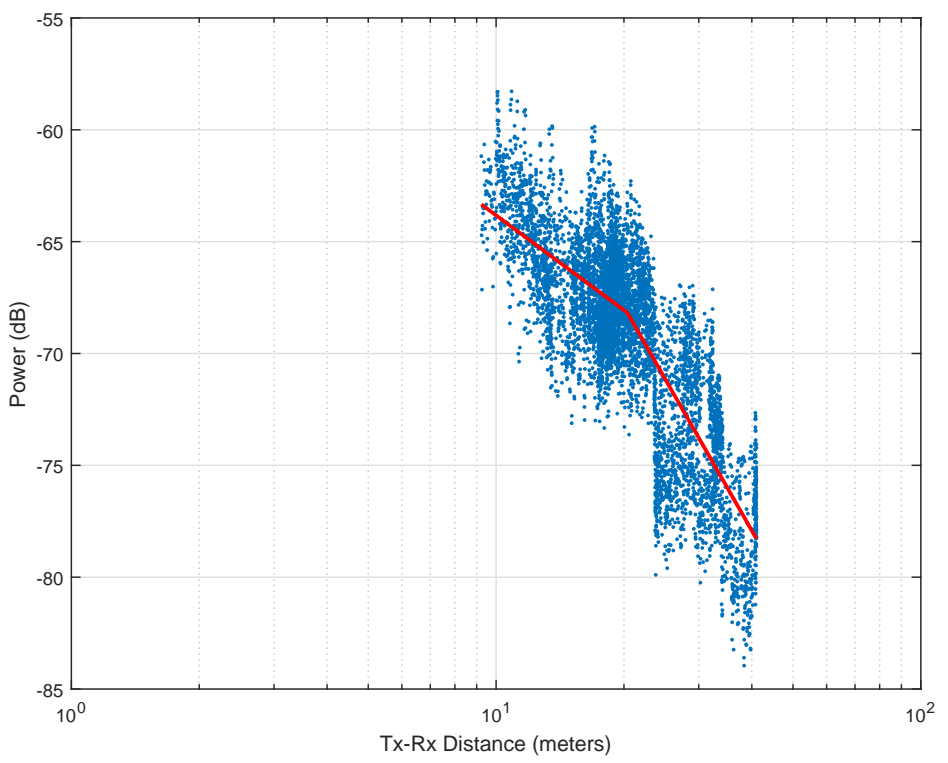

Figure 22: Non Line of Sight Received Power vs. Distance: Cross Polarization, 2.245GHz, T1. 


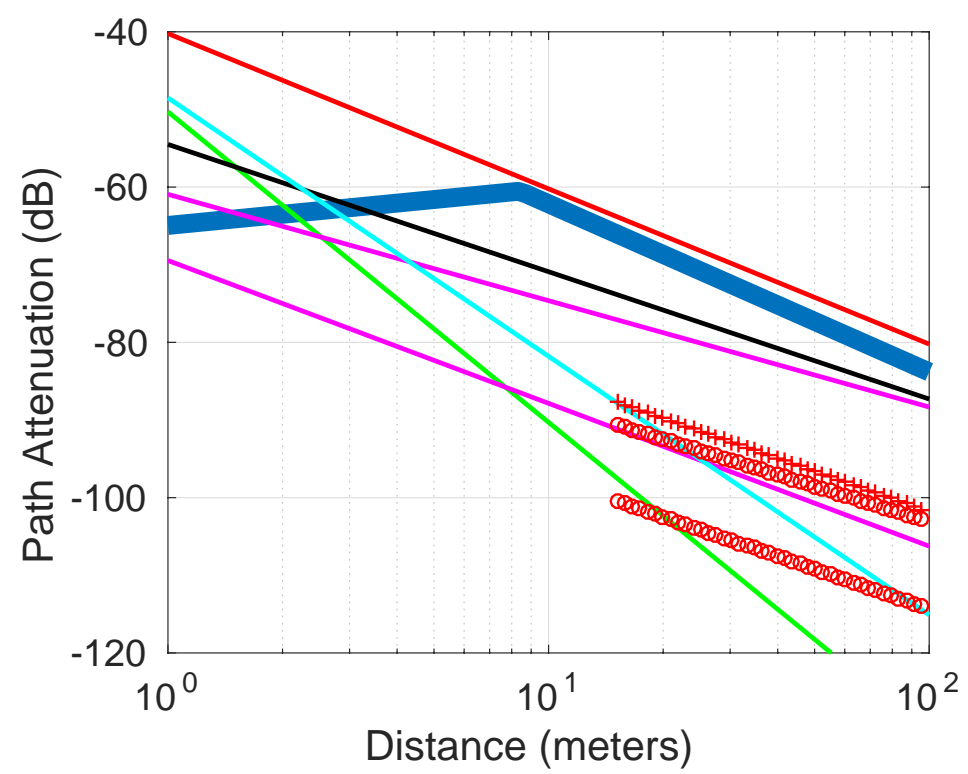

Figure 23: Comparison to other models: Cross Polarization, 2.245GHz, T1.

\begin{tabular}{c|c|c|c|c|c|c|c|c}
\hline Type & Frequency & Polarization & $\alpha_{1}$ & $\alpha_{2}$ & $\beta(\mathrm{m})$ & $k_{1}(\mathrm{~dB})$ & $k_{2}(\mathrm{~dB})$ & MSE \\
\hline \hline Overall & $2.245 \mathrm{GHz}$ & CrossPol & -0.47776 & 2.166 & 8.4098 & -64.9408 & -40.4919 & 9.3129 \\
\hline LOS & $2.245 \mathrm{GHz}$ & CrossPol & -0.48848 & 1.6744 & 7.8537 & -64.8572 & -45.4973 & 9.5928 \\
\hline NLOS & $2.245 \mathrm{GHz}$ & CrossPol & 1.4082 & 3.3221 & 20.3506 & -49.7419 & -24.6974 & 8.0049 \\
\hline
\end{tabular}

Table 2: Parameters for Cross Polarization, 2.245GHz, T1 


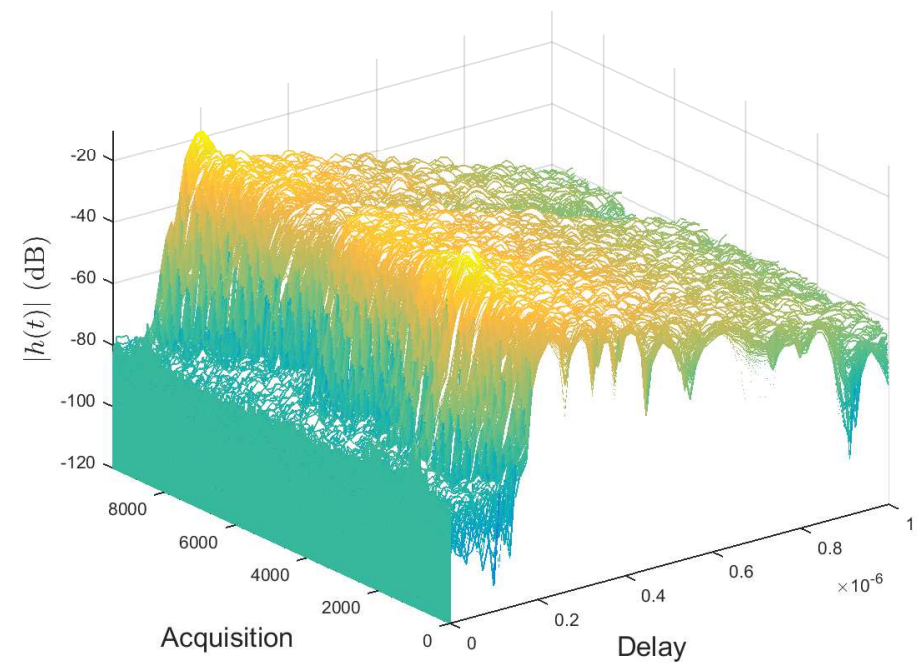

Figure 24: Impulse Responses: Cross Polarization, 2.245GHz, T1.

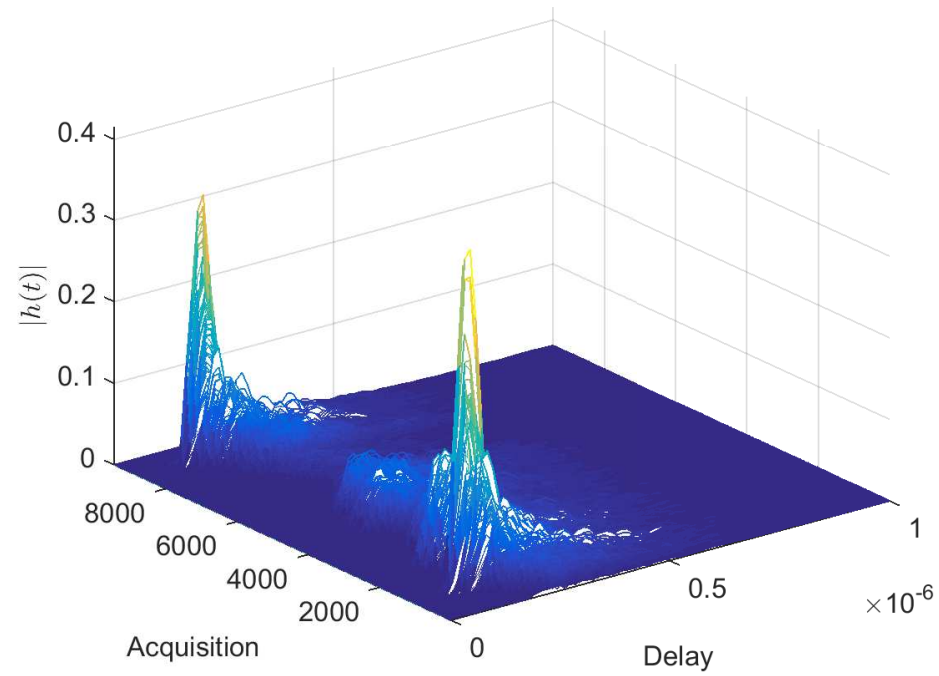

Figure 25: Impulse Responses: Cross Polarization, 2.245GHz, T1. 


\section{A.2 Cross Polarization, 5GHz, Transmitter 1}

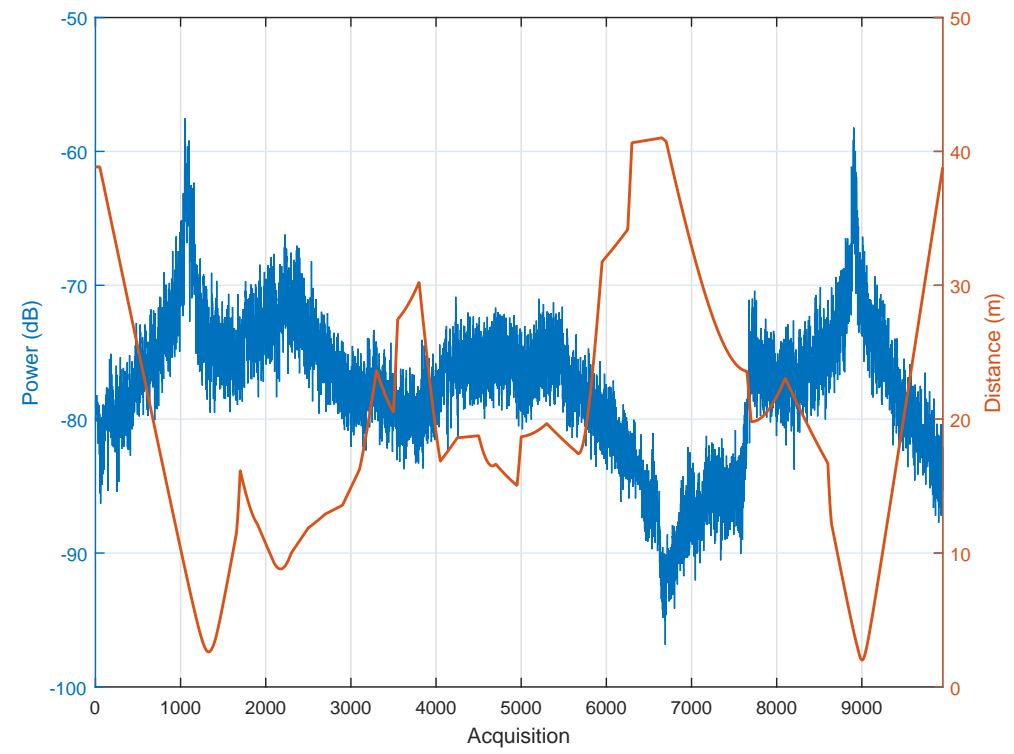

Figure 26: Overall Received Power vs. Acquisition Number and Distance vs. Acquisition Number: Cross Polarization, 5.4GHz, T1.

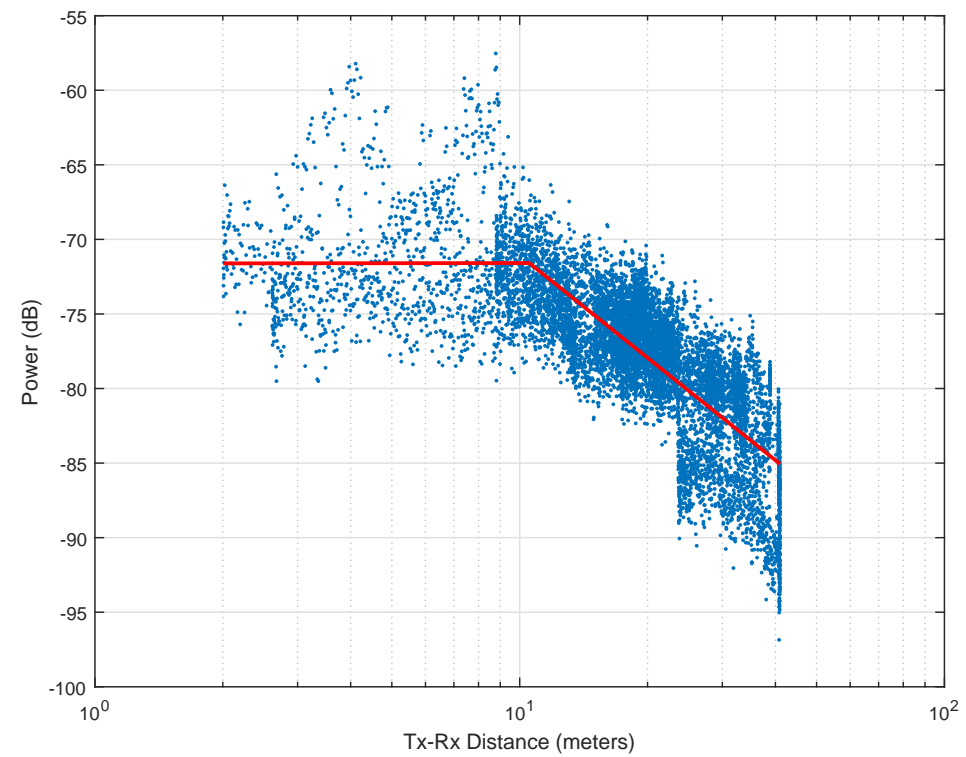

Figure 27: Overall Received Power vs. Distance: Cross Polarization, 5.4GHz, T1. 


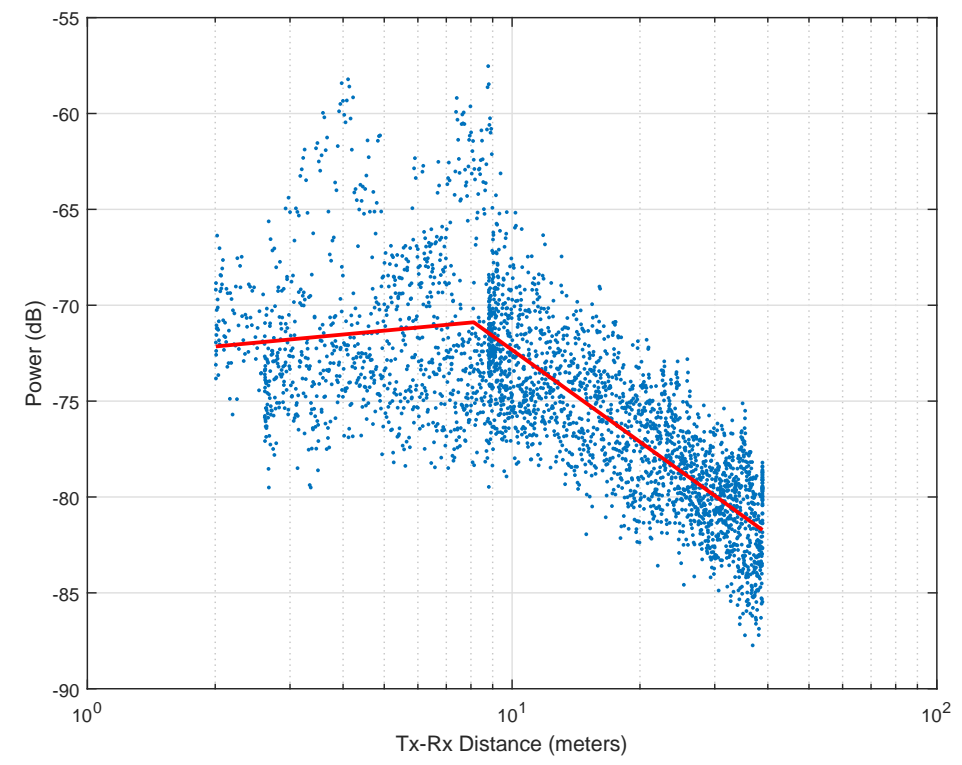

Figure 28: Line of Sight Received Power vs. Distance: Cross Polarization, 5.4GHz, T1.

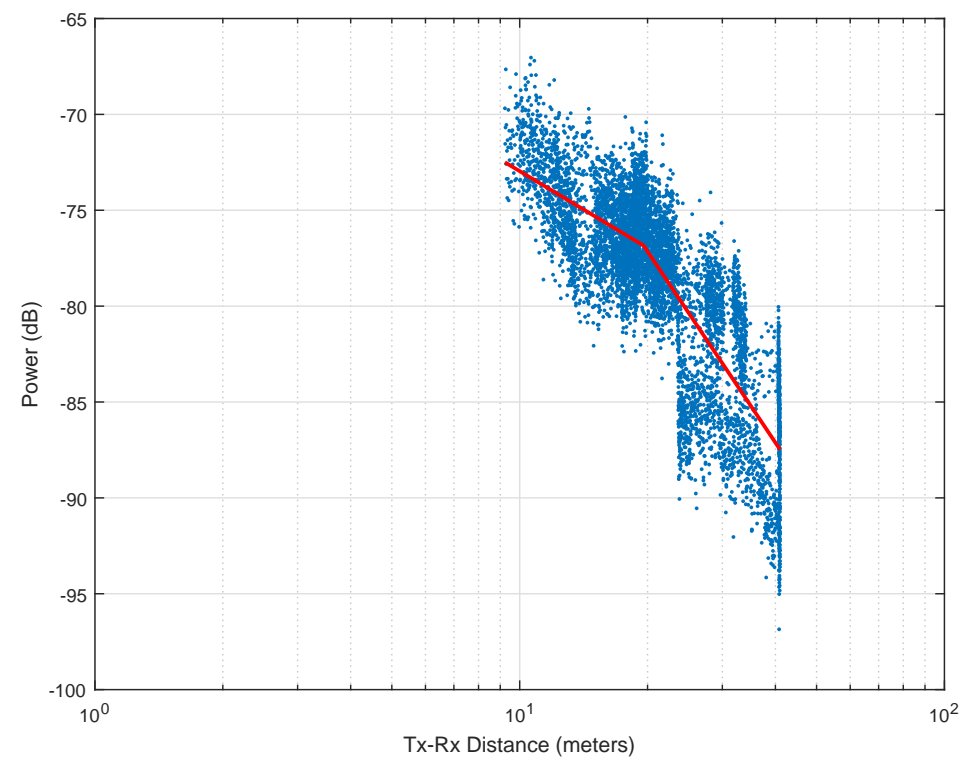

Figure 29: Non Line of Sight Received Power vs. Distance: Cross Polarization, 5.4GHz, $\mathrm{T} 1$. 


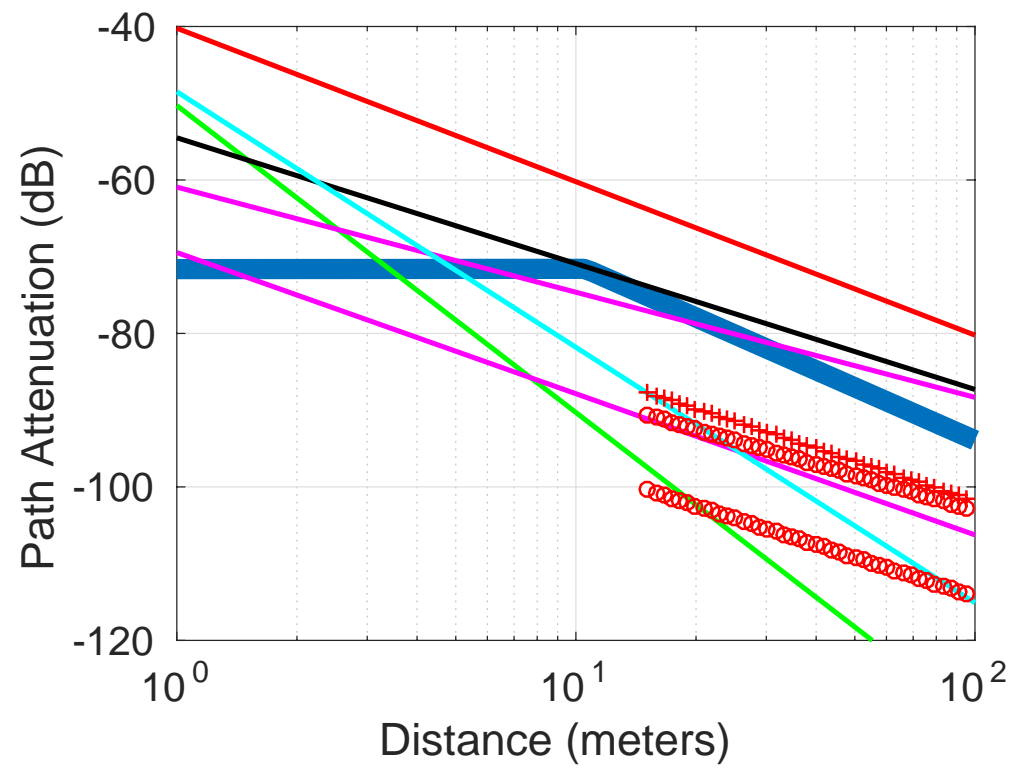

Figure 30: Comparison to other models: Cross Polarization, 5.4GHz, T1.

\begin{tabular}{c|c|c|c|c|c|c|c|c}
\hline Type & Frequency & Polarization & $\alpha_{1}$ & $\alpha_{2}$ & $\beta(\mathrm{m})$ & $k_{1}(\mathrm{~dB})$ & $k_{2}(\mathrm{~dB})$ & MSE \\
\hline \hline Overall & $5.4 \mathrm{GHz}$ & CrossPol & -0.0035534 & 2.2885 & 10.5681 & -71.6171 & -48.1464 & 9.8974 \\
\hline LOS & $5.4 \mathrm{GHz}$ & CrossPol & -0.20753 & 1.5937 & 8.1212 & -72.7759 & -56.3919 & 10.1328 \\
\hline NLOS & $5.4 \mathrm{GHz}$ & CrossPol & 1.3306 & 3.3114 & 19.5742 & -59.6536 & -34.0677 & 8.8339 \\
\hline
\end{tabular}

Table 3: Parameters for Cross Polarization, 5.4GHz, T1 


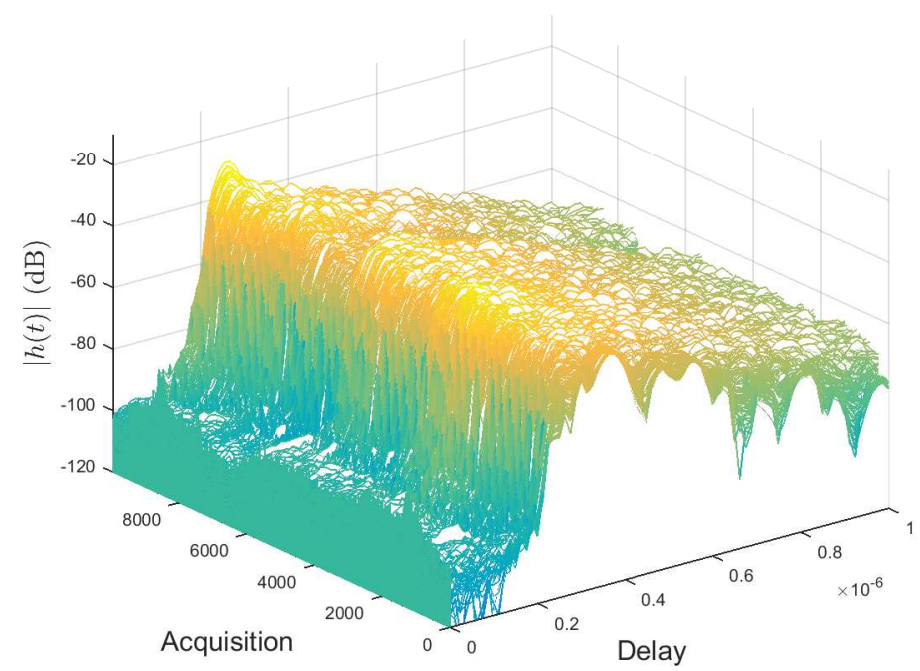

Figure 31: Impulse Responses: Cross Polarization, 5.4GHz, T1.

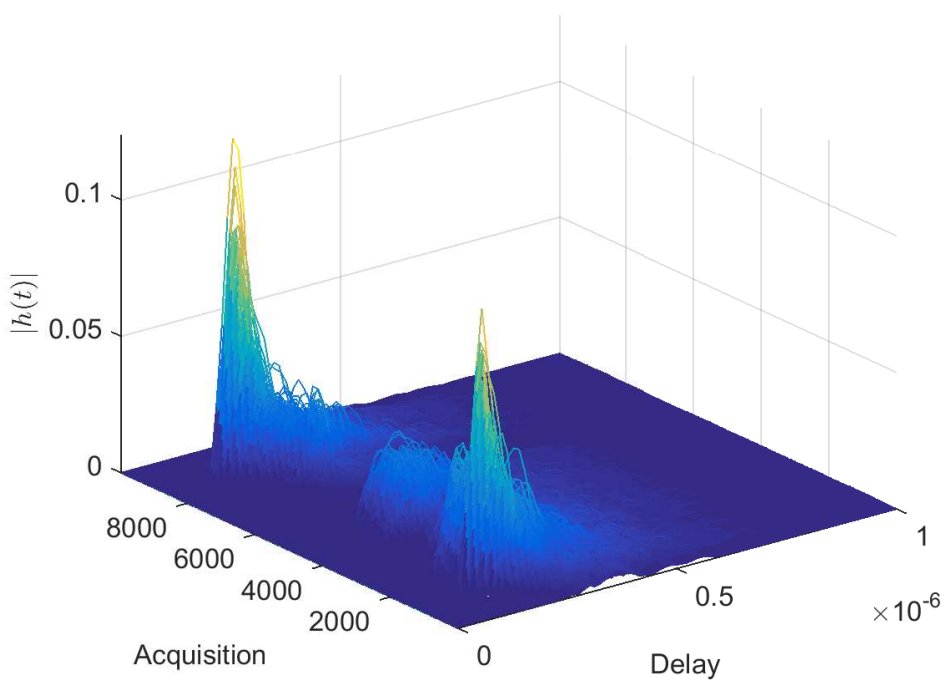

Figure 32: Impulse Responses: Cross Polarization, 5.4GHz, T1. 


\section{A.3 Cross Polarization, 2.245GHz, Transmitter 2, High}

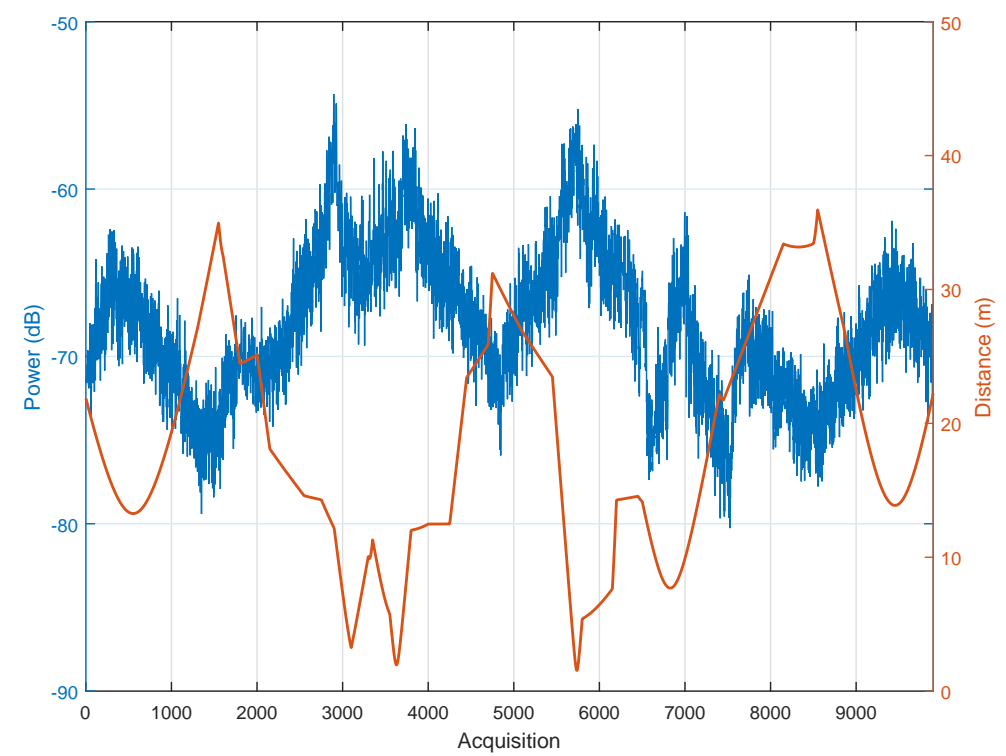

Figure 33: Overall Received Power vs. Acquisition Number and Distance vs. Acquisition Number: Cross Polarization, 2.245GHz, T2, High.

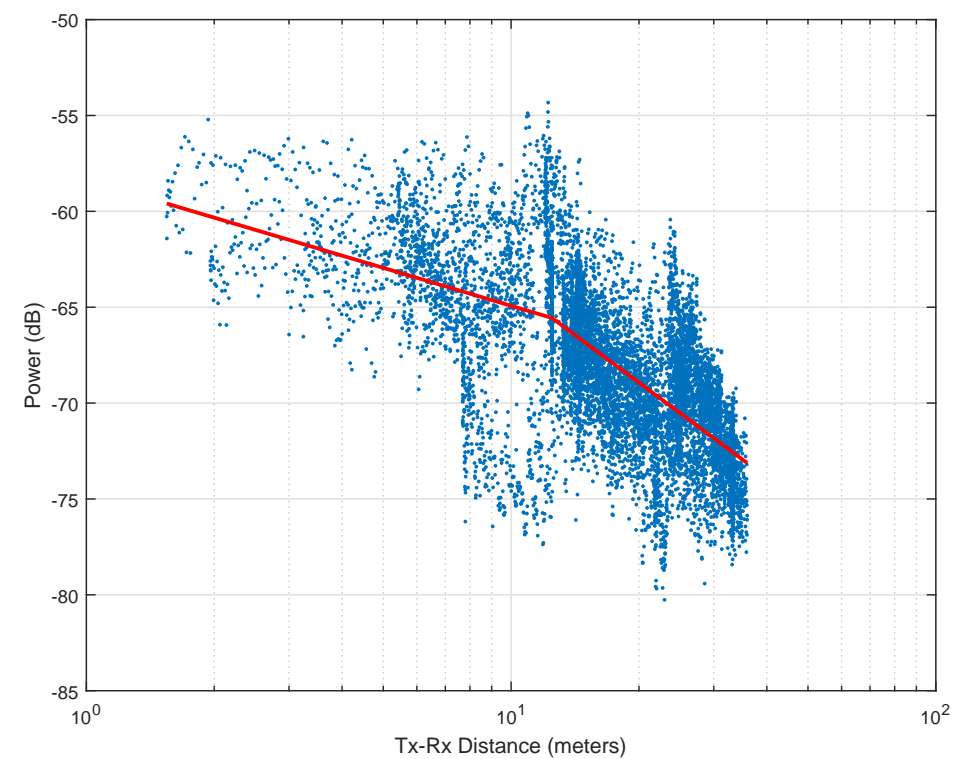

Figure 34: Overall Received Power vs. Distance: Cross Polarization, 2.245GHz, T2, High. 


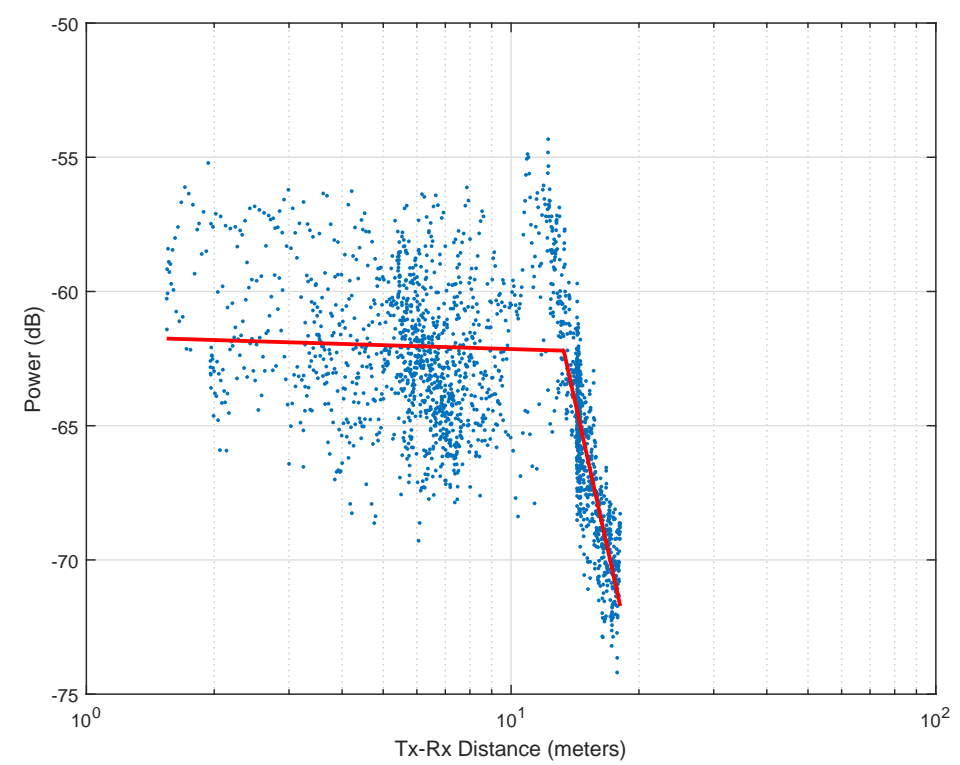

Figure 35: Line of Sight Received Power vs. Distance: Cross Polarization, 2.245GHz, T2, High.

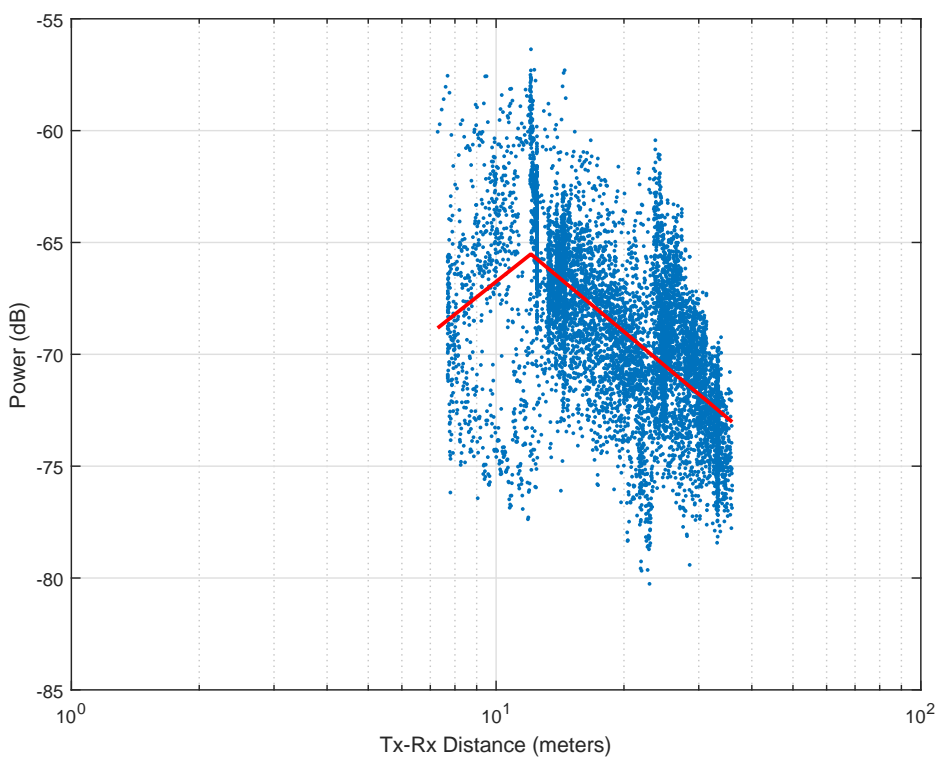

Figure 36: Non Line of Sight Received Power vs. Distance: Cross Polarization, 2.245GHz, T2, High. 


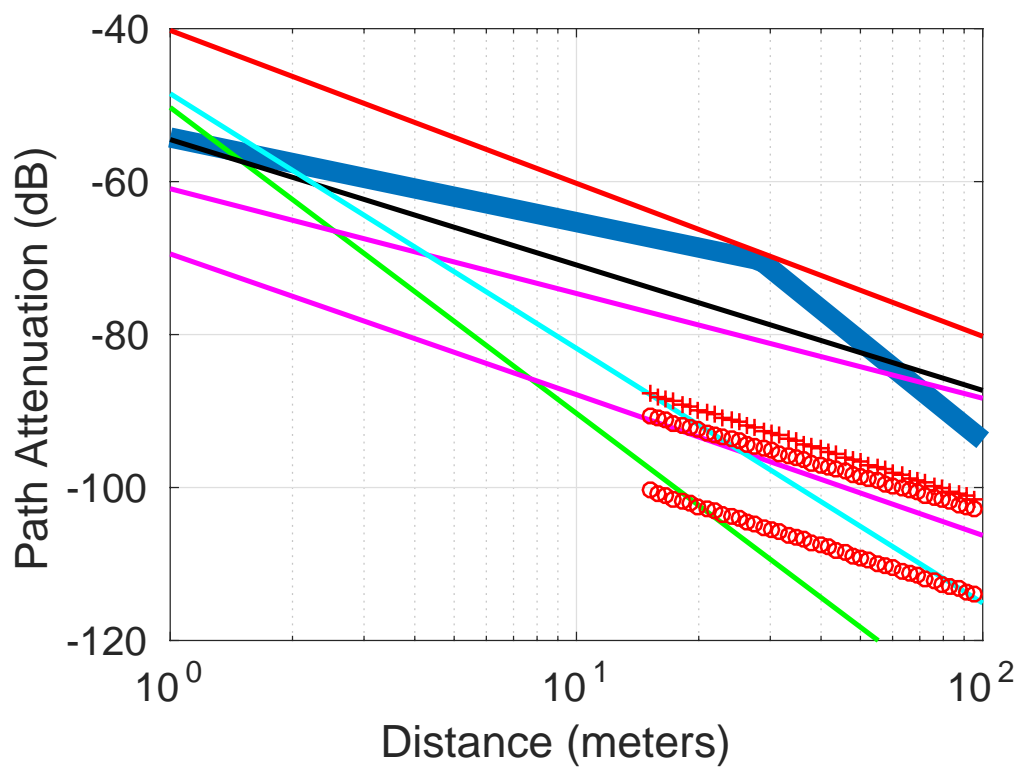

Figure 37: Comparison to other models: Cross Polarization, 2.245GHz, T2, High.

\begin{tabular}{c|c|c|c|c|c|c|c|c}
\hline Type & Frequency & Polarization & $\alpha_{1}$ & $\alpha_{2}$ & $\beta(\mathrm{m})$ & $k_{1}(\mathrm{~dB})$ & $k_{2}(\mathrm{~dB})$ & MSE \\
\hline \hline Overall & $2.245 \mathrm{GHz}$ & CrossPol High & 1.1065 & 4.3014 & 28.4304 & -54.2346 & -7.7875 & 10.2871 \\
\hline LOS & $2.245 \mathrm{GHz}$ & CrossPol High & 0.048162 & 7.1472 & 13.3019 & -61.6634 & 18.1234 & 7.6617 \\
\hline NLOS & $2.245 \mathrm{GHz}$ & CrossPol High & -1.5062 & 1.5834 & 12.0752 & -81.815 & -48.3886 & 9.8115 \\
\hline
\end{tabular}

Table 4: Parameters for Cross Polarization, 2.245GHz, T1, High 


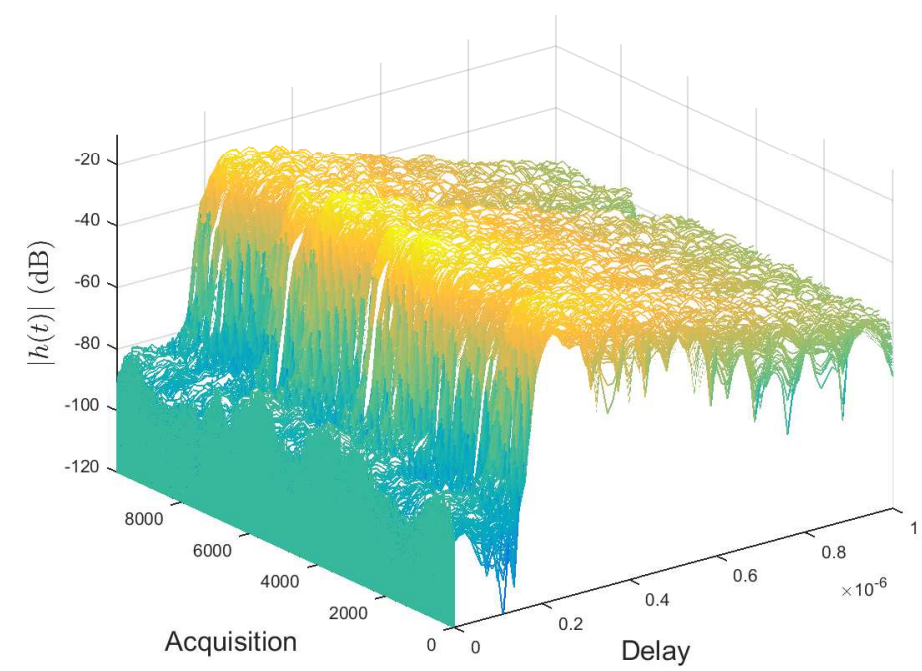

Figure 38: Impulse Responses: Cross Polarization, 2.245GHz, T2, High.

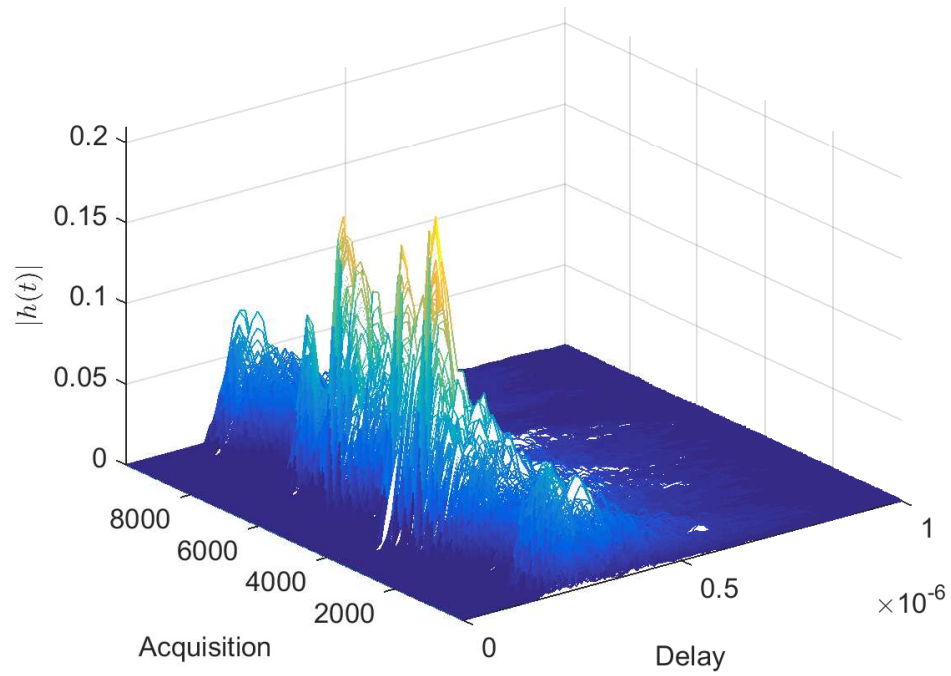

Figure 39: Impulse Responses: Cross Polarization, 2.245GHz, T2, High. 


\section{A.4 Cross Polarization, 2.245GHz, Transmitter 2, Low}

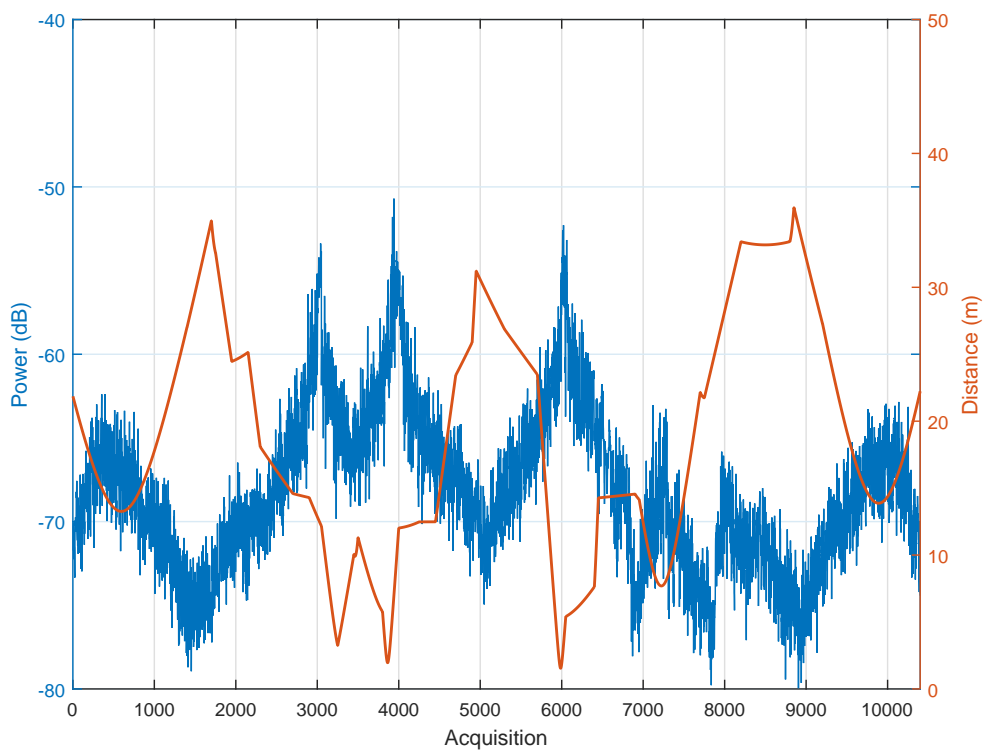

Figure 40: Overall Received Power vs. Acquisition Number and Distance vs. Acquisition Number: Cross Polarization, 2.245GHz, T2, Low.

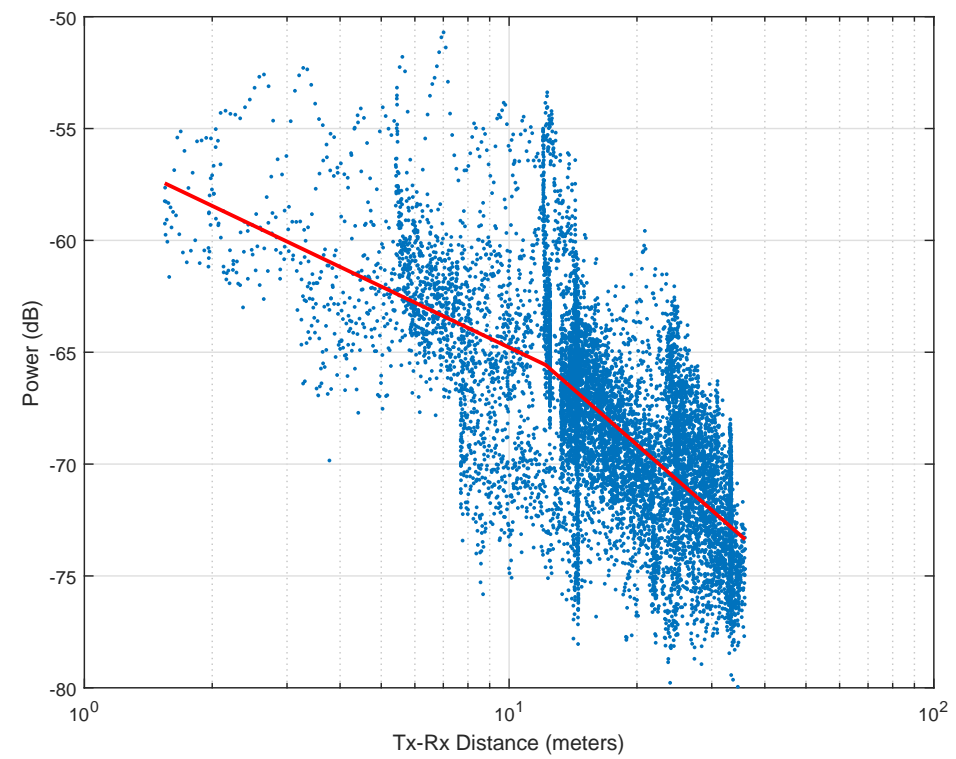

Figure 41: Overall Received Power vs. Distance: Cross Polarization, 2.245GHz, T2, Low. 


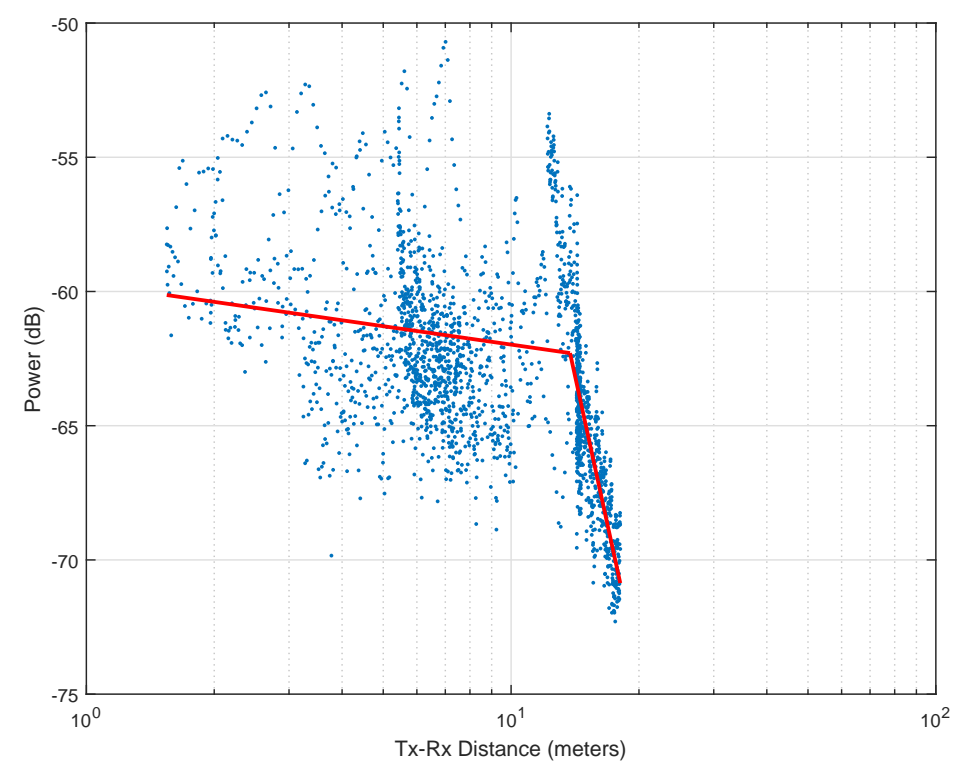

Figure 42: Line of Sight Received Power vs. Distance: Cross Polarization, $2.245 \mathrm{GHz}$, T2, Low.

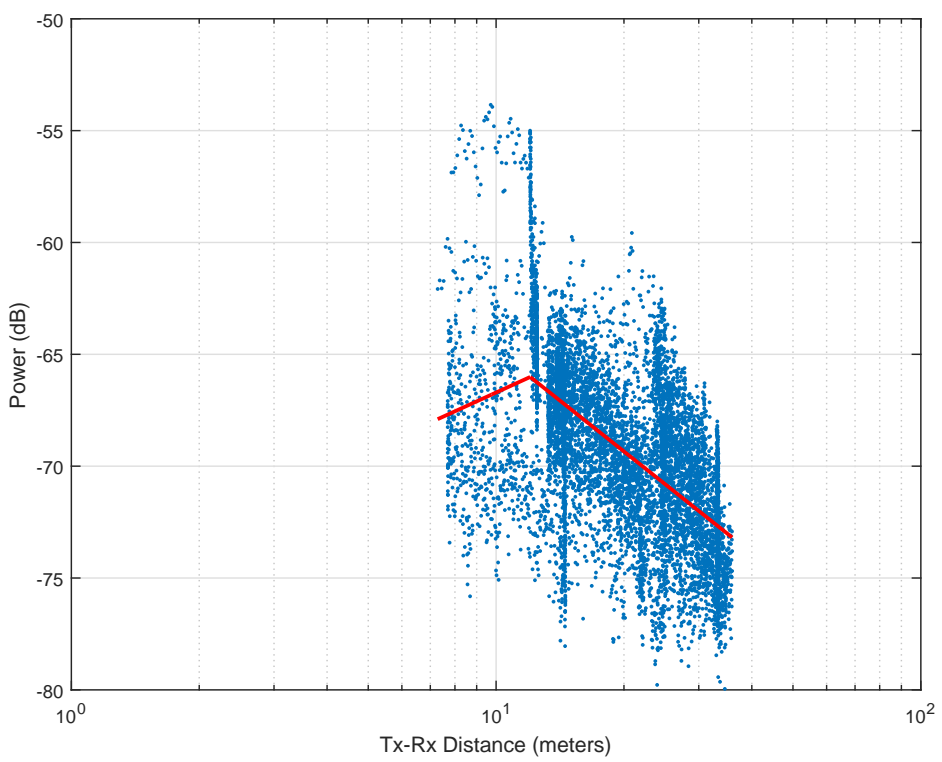

Figure 43: Non Line of Sight Received Power vs. Distance: Cross Polarization, 2.245GHz, T2, Low. 


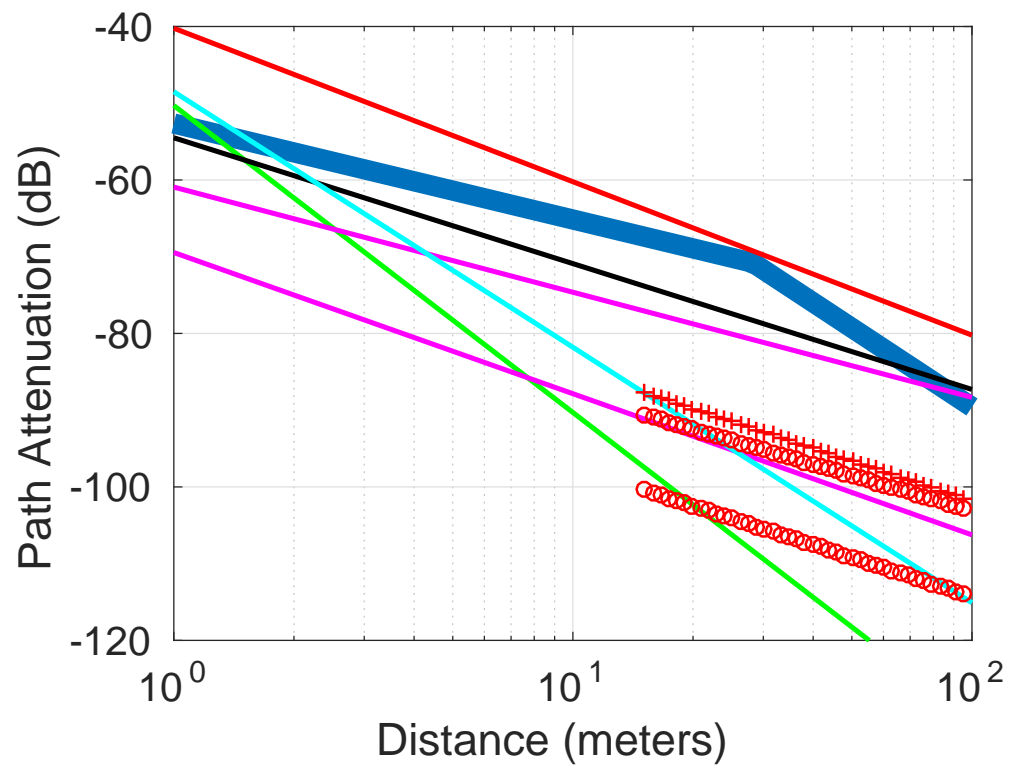

Figure 44: Comparison to other models: Cross Polarization, 2.245GHz, T2, Low.

\begin{tabular}{c|c|c|c|c|c|c|c|c}
\hline Type & Frequency & Polarization & $\alpha_{1}$ & $\alpha_{2}$ & $\beta(\mathrm{m})$ & $k_{1}(\mathrm{~dB})$ & $k_{2}(\mathrm{~dB})$ & MSE $(\mathrm{dB})$ \\
\hline \hline Overall & $2.245 \mathrm{GHz}$ & CrossPol Low & 1.251 & 3.4239 & 28.266 & -52.6428 & -21.1079 & 10.4894 \\
\hline LOS & $2.245 \mathrm{GHz}$ & CrossPol Low & -0.86565 & 1.5089 & 12.0315 & -75.3644 & -49.7118 & 10.0073 \\
\hline NLOS & $2.245 \mathrm{GHz}$ & CrossPol Low & -0.86565 & 1.5089 & 12.0315 & -75.3644 & -49.7118 & 10.0073 \\
\hline
\end{tabular}

Table 5: Parameters for Cross Polarization, 2.245GHz, T2, Low 


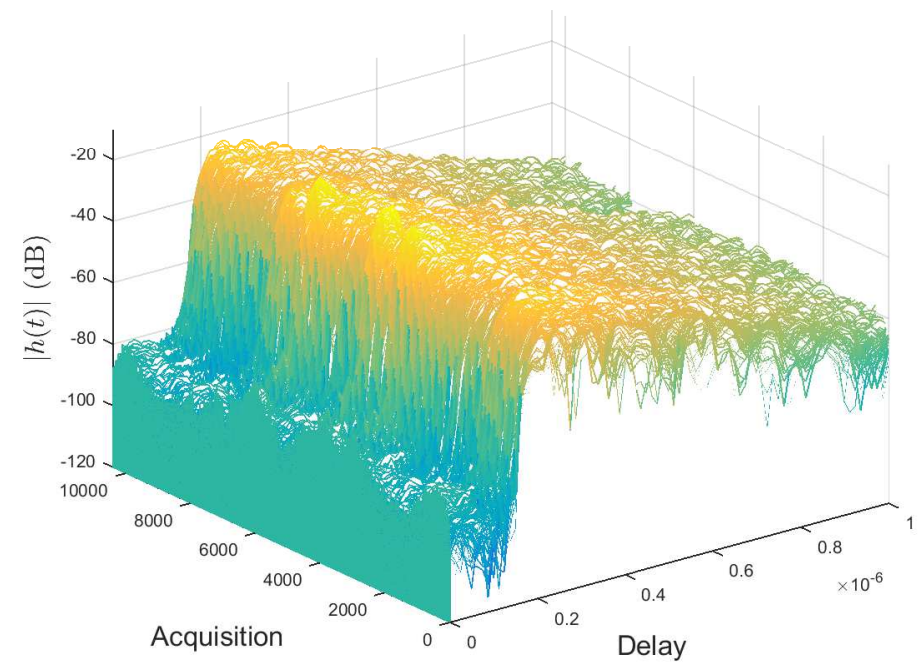

Figure 45: Impulse Responses: Cross Polarization, 2.245GHz, T2, Low.

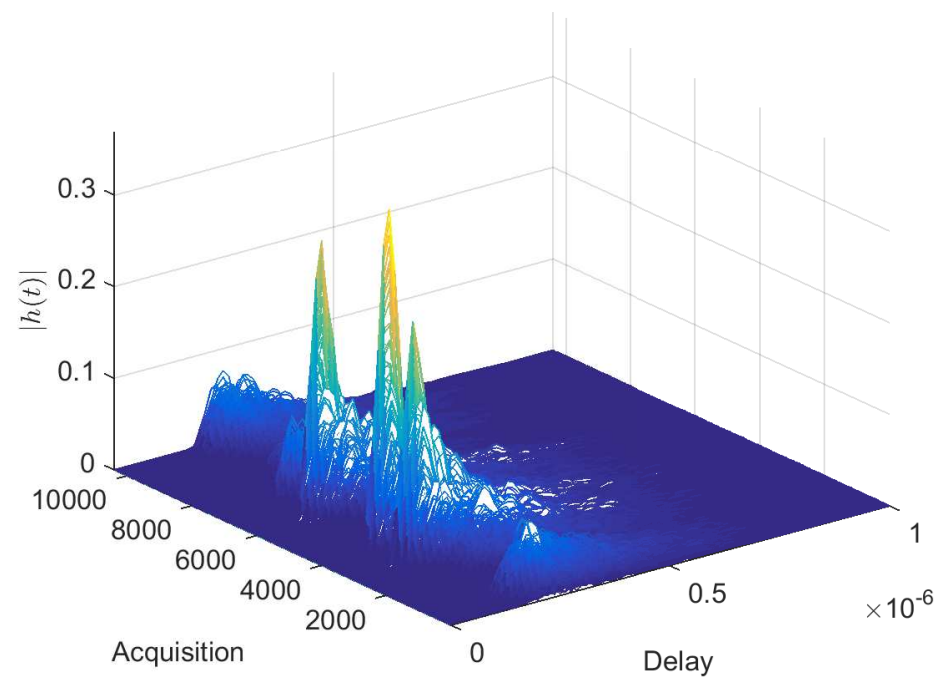

Figure 46: Impulse Responses: Cross Polarization, 2.245GHz, T2, Low. 


\section{A.5 Cross Polarization, 5GHz, Transmitter 2, High}

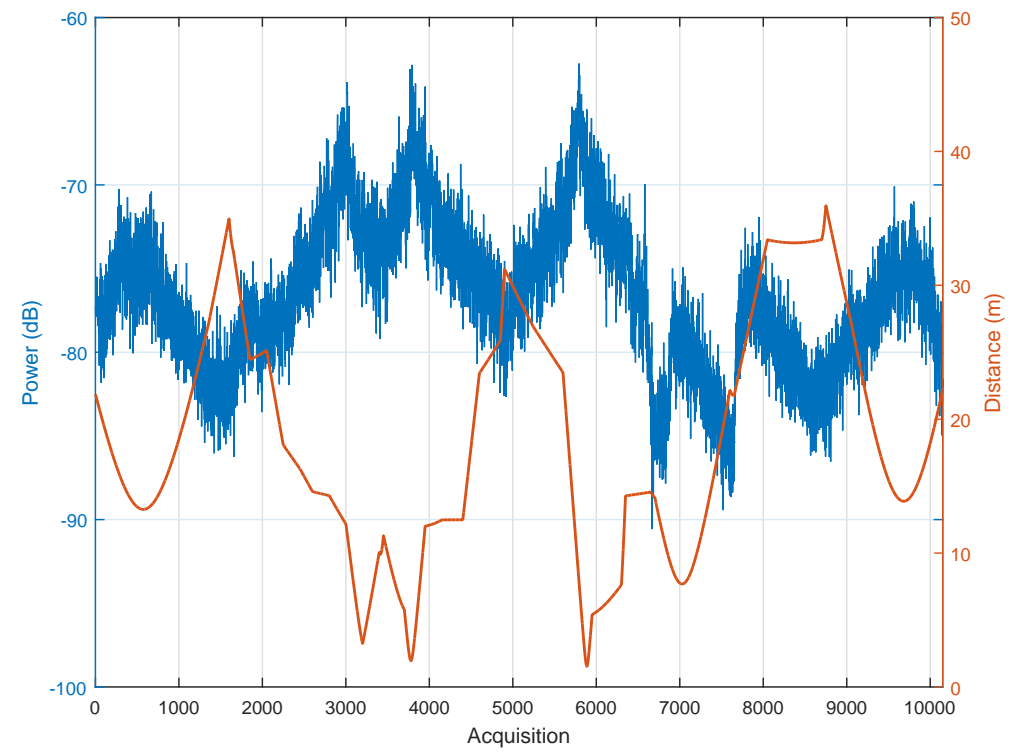

Figure 47: Overall Received Power vs. Acquisition Number and Distance vs. Acquisition Number: Cross Polarization, 5.4GHz, T2, High.

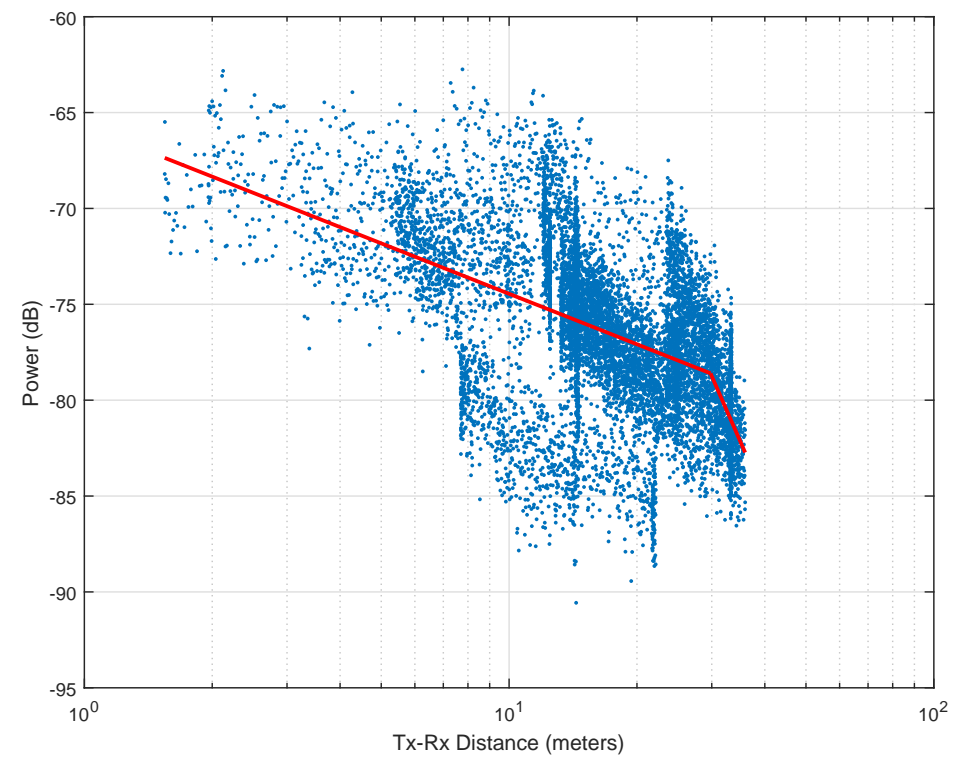

Figure 48: Overall Received Power vs. Distance: Cross Polarization, 5.4GHz, T2, High. 


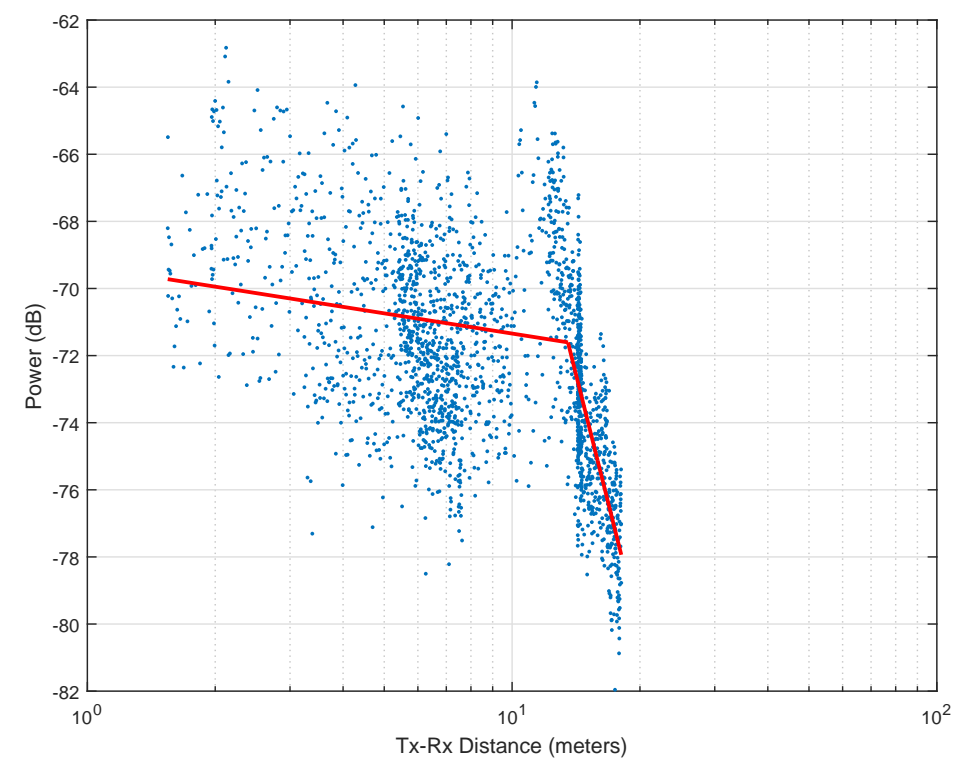

Figure 49: Line of Sight Received Power vs. Distance: Cross Polarization, 5.4GHz, T2, High.

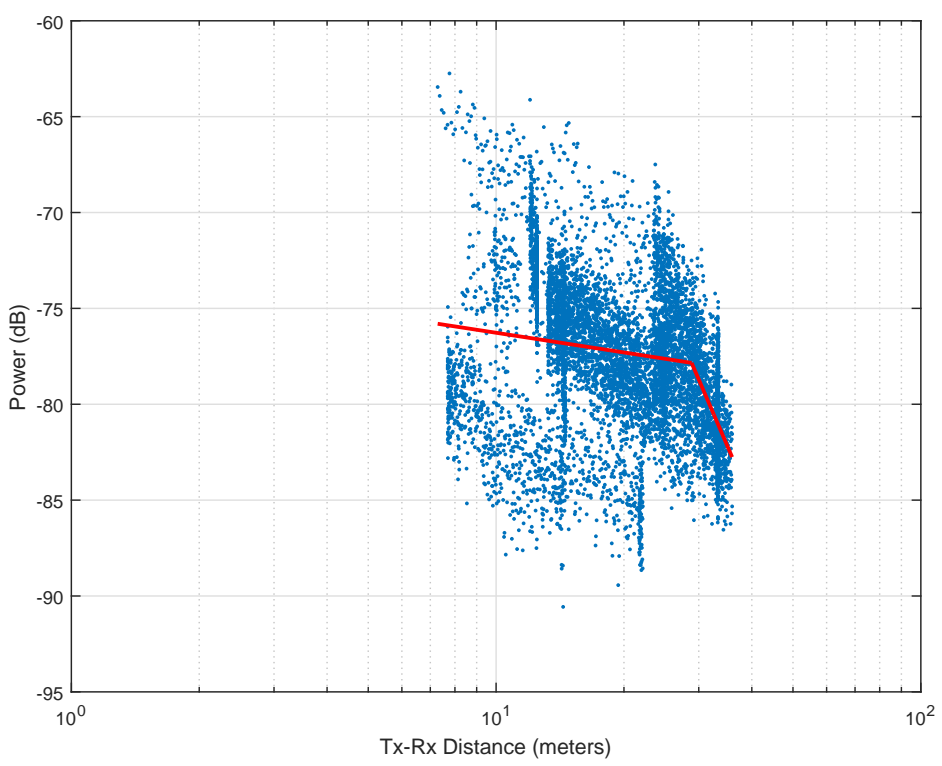

Figure 50: Non Line of Sight Received Power vs. Distance: Cross Polarization, 5.4GHz, T2, High. 


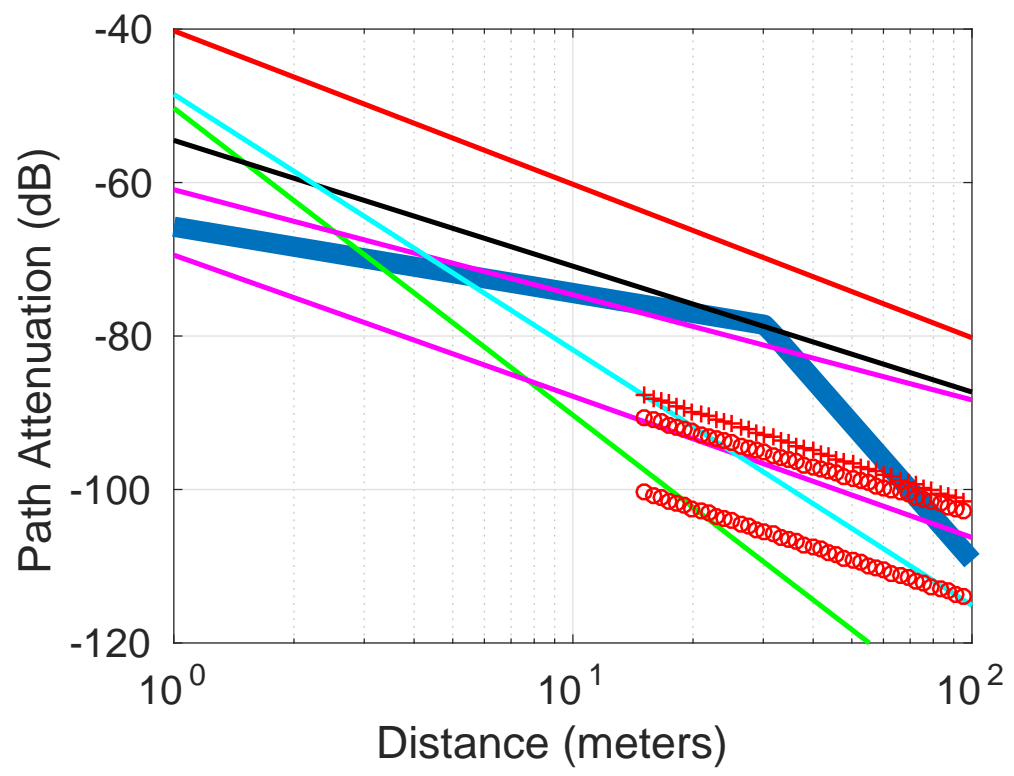

Figure 51: Comparison to other models: Cross Polarization, 5.4GHz, T2, High.

\begin{tabular}{c|c|c|c|c|c|c|c|c}
\hline Type & Frequency & Polarization & $\alpha_{1}$ & $\alpha_{2}$ & $\beta(\mathrm{m})$ & $k_{1}(\mathrm{~dB})$ & $k_{2}(\mathrm{~dB})$ & MSE \\
\hline \hline Overall & $2.245 \mathrm{GHz}$ & CrossPol High & 1.1065 & 4.3014 & 28.4304 & -54.2346 & -7.7875 & 10.2871 \\
\hline LOS & $2.245 \mathrm{GHz}$ & CrossPol High & 0.048162 & 7.1472 & 13.3019 & -61.6634 & 18.1234 & 7.6617 \\
\hline NLOS & $2.245 \mathrm{GHz}$ & CrossPol High & -1.5062 & 1.5834 & 12.0752 & -81.815 & -48.3886 & 9.8115 \\
\hline
\end{tabular}

Table 6: Parameters for Cross Polarization, 2.245GHz, T2, High 


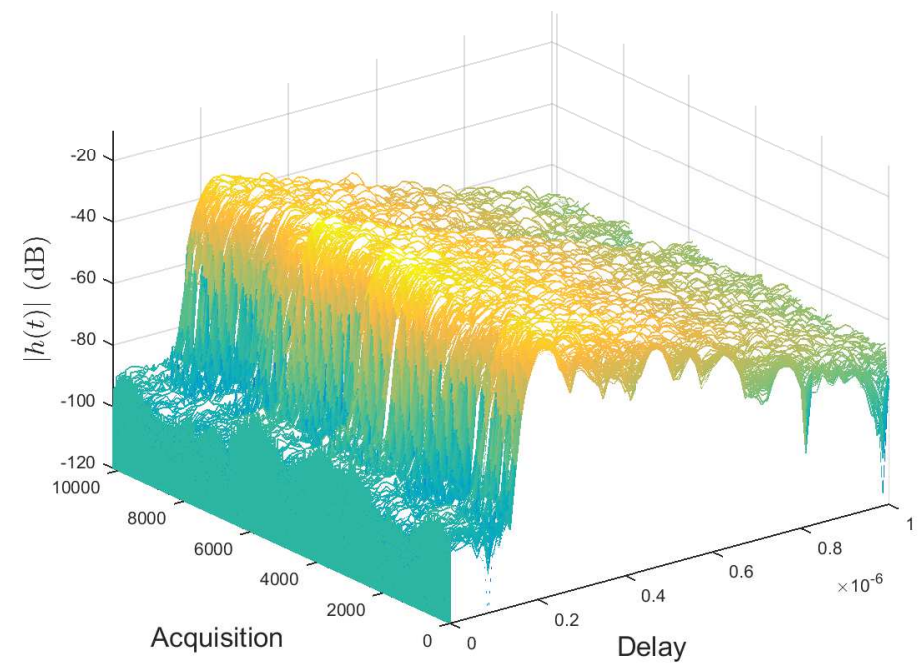

Figure 52: Impulse Responses: Cross Polarization, 5.4GHz, T2, High.

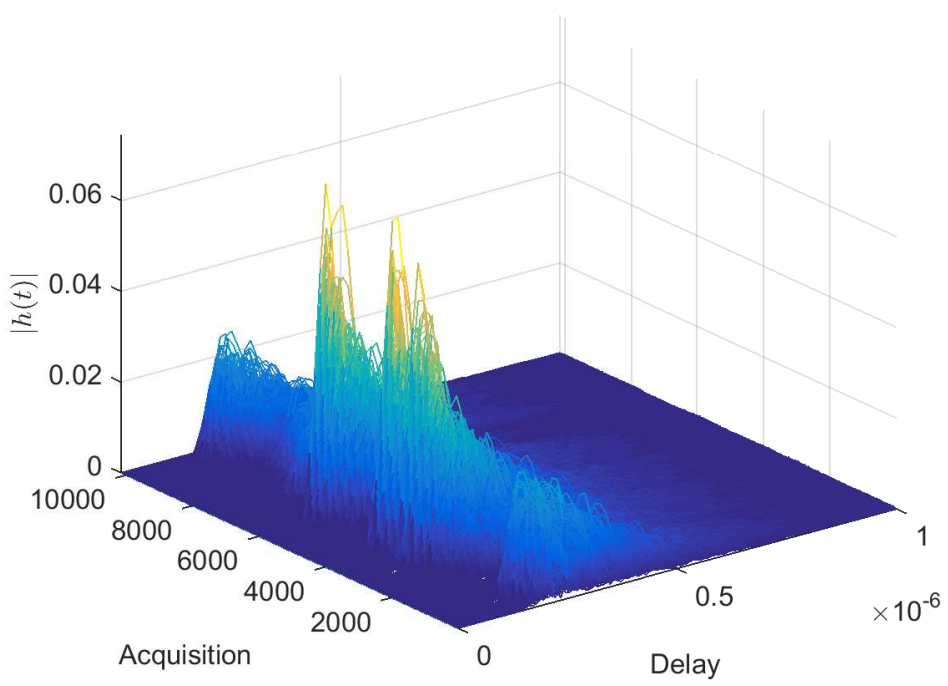

Figure 53: Impulse Responses: Cross Polarization, 5.4GHz, T2, High. 


\section{A.6 Cross Polarization, 5GHz, Transmitter 2, Low}

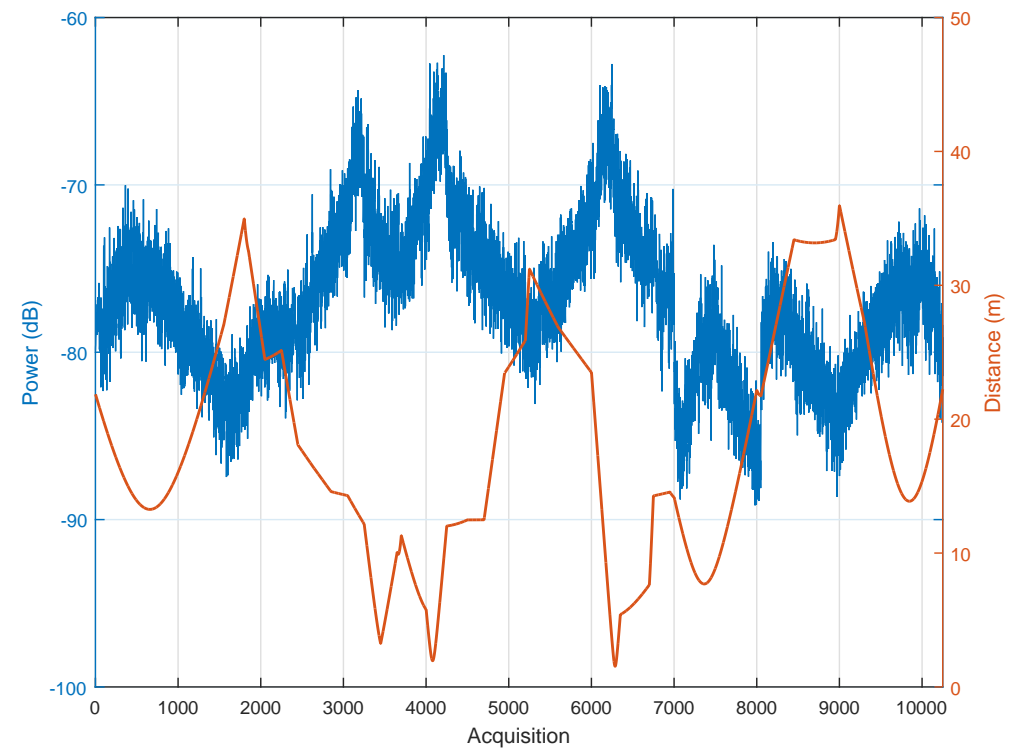

Figure 54: Overall Received Power vs. Acquisition Number and Distance vs. Acquisition Number: Cross Polarization, 5.4GHz, T2, Low.

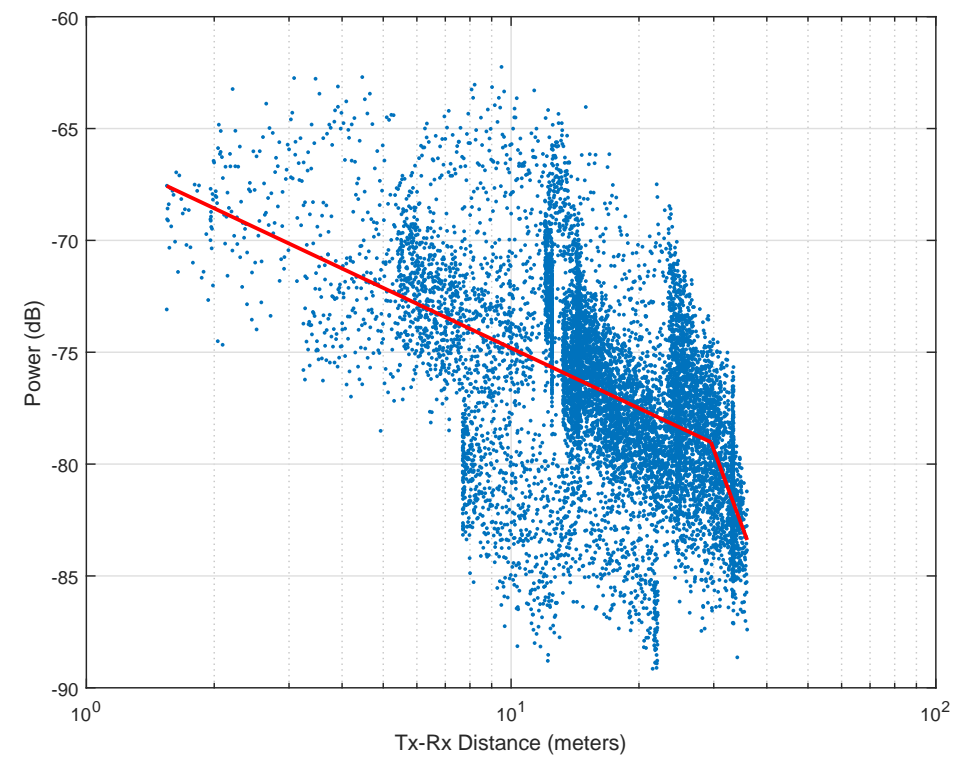

Figure 55: Overall Received Power vs. Distance: Cross Polarization, 5.4GHz, T2, Low. 


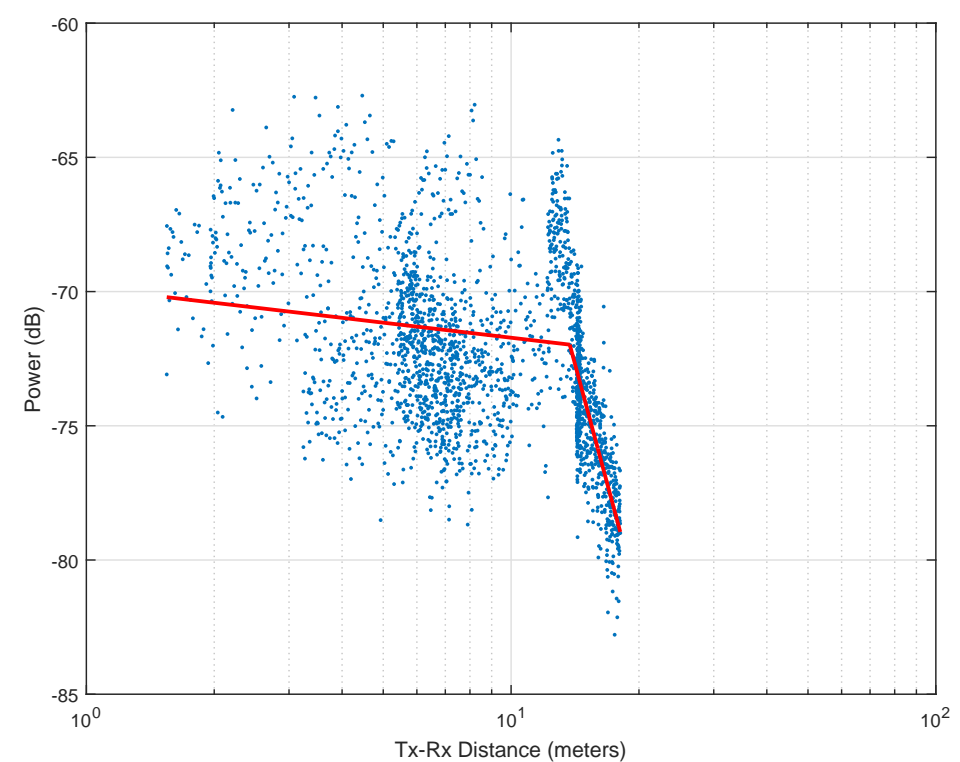

Figure 56: Line of Sight Received Power vs. Distance: Cross Polarization, 5.4GHz, T2, Low.

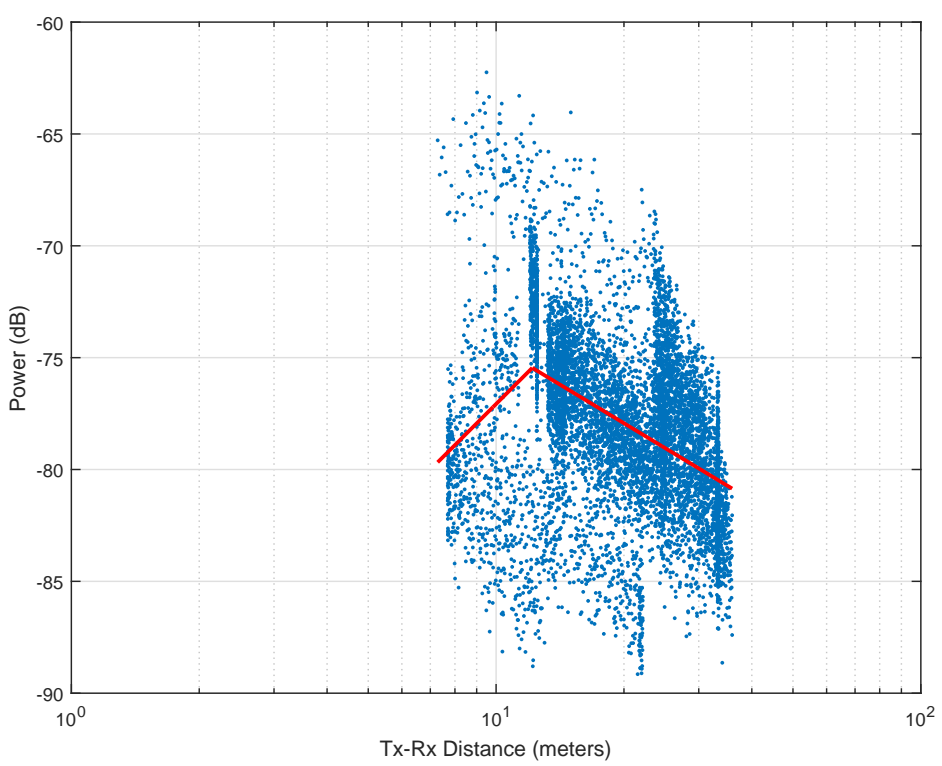

Figure 57: Non Line of Sight Received Power vs. Distance: Cross Polarization, 5.4GHz, T2, Low. 


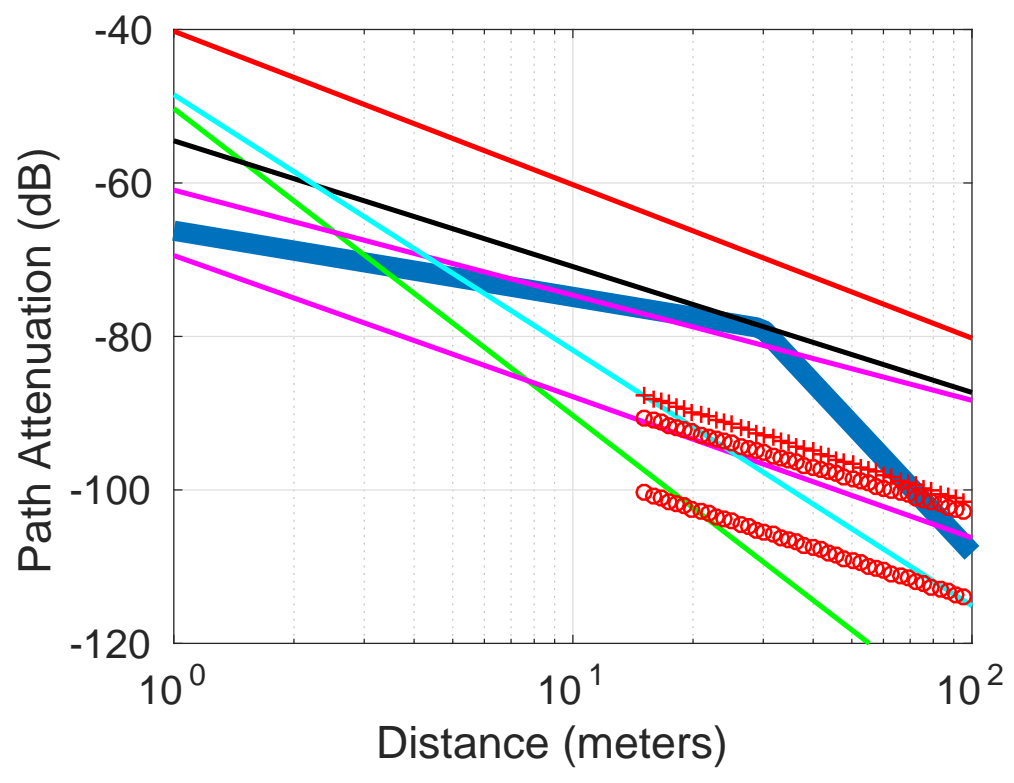

Figure 58: Comparison to other models: Cross Polarization, 5.4GHz, T2, Low.

\begin{tabular}{c|c|c|c|c|c|c|c|c}
\hline Type & Frequency & Polarization & $\alpha_{1}$ & $\alpha_{2}$ & $\beta(\mathrm{m})$ & $k_{1}(\mathrm{~dB})$ & $k_{2}(\mathrm{~dB})$ & MSE \\
\hline \hline Overall & $5.4 \mathrm{GHz}$ & CrossPol Low & 0.8628 & 5.5696 & 29.8334 & -66.2216 & 3.1899 & 11.5172 \\
\hline LOS & $5.4 \mathrm{GHz}$ & CrossPol Low & 0.18635 & 5.8664 & 13.743 & -69.8559 & -5.2128 & 8.6103 \\
\hline NLOS & $5.4 \mathrm{GHz}$ & CrossPol Low & -1.8951 & 1.1466 & 12.1666 & -96.0213 & -63.0146 & 11.1937 \\
\hline
\end{tabular}

Table 7: Parameters for Cross Polarization, 5GHz, T2, Low 


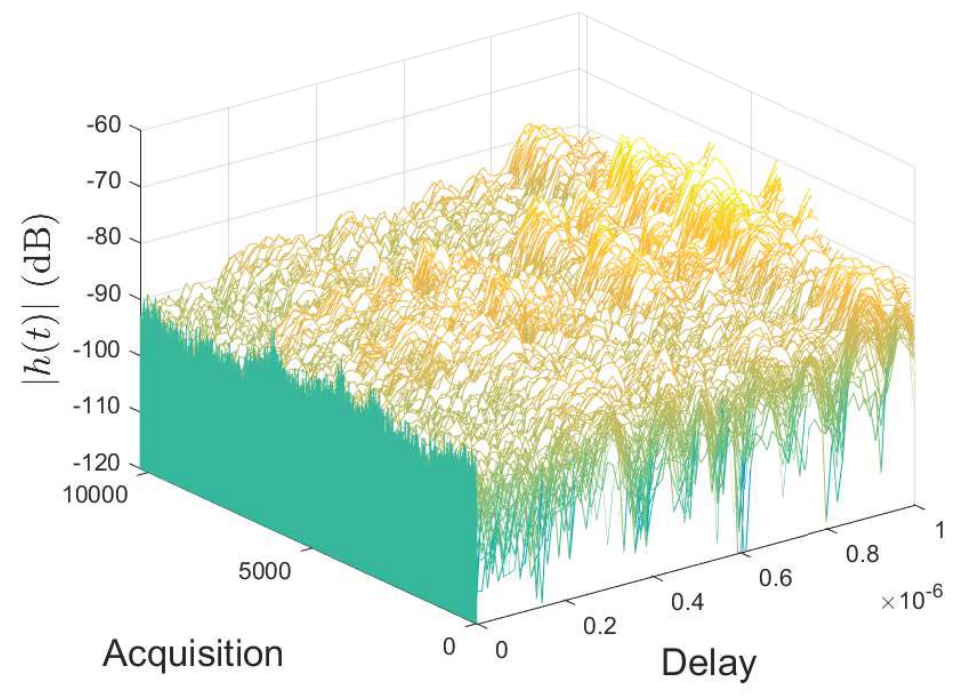

Figure 59: Impulse Responses: Cross Polarization, 5.4GHz, T2, Low.

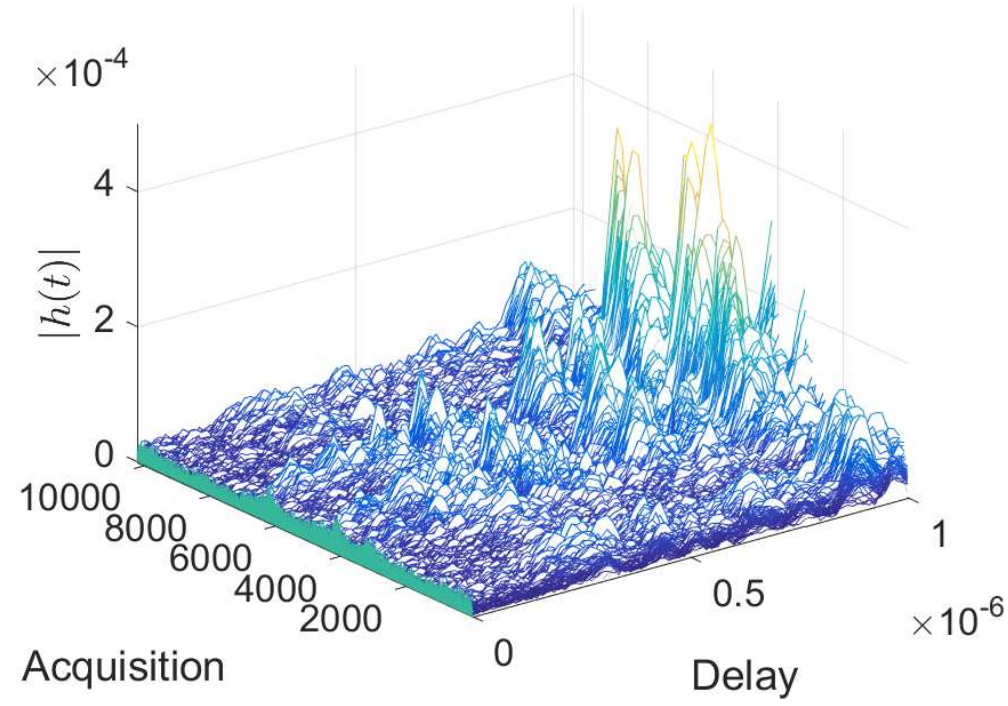

Figure 60: Impulse Responses: Cross Polarization, 5.4GHz, T2, Low. 


\section{A.7 Long Polarization, 2.245GHz, Transmitter 1}

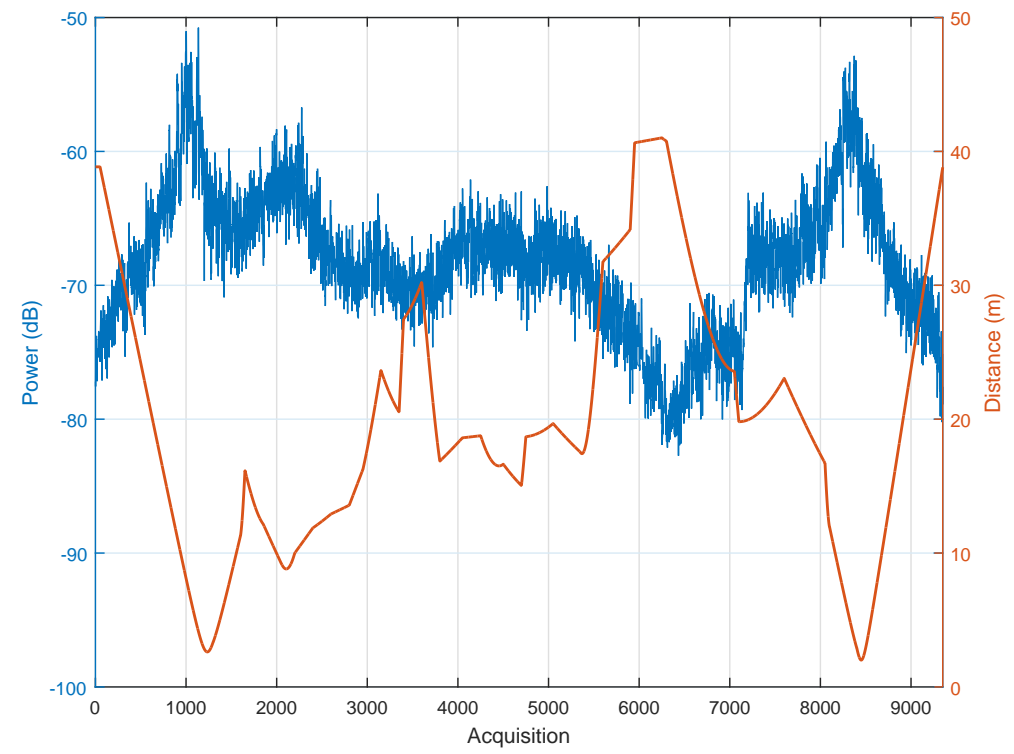

Figure 61: Overall Received Power vs. Acquisition Number and Distance vs. Acquisition Number: Long Polarization, 2.245GHz, T1.

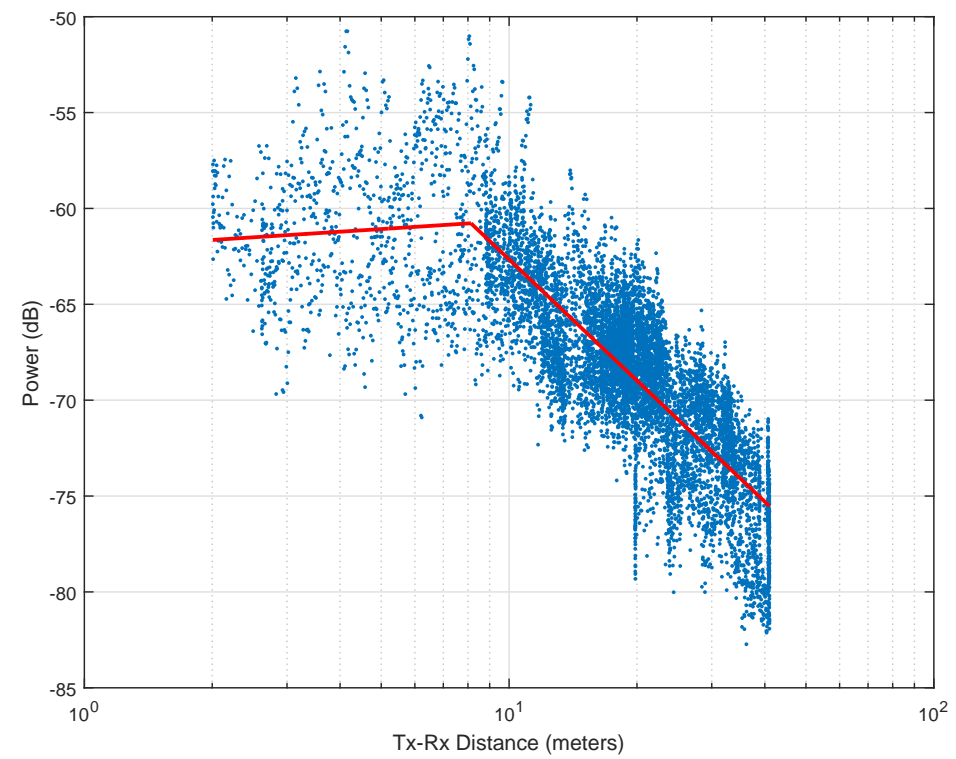

Figure 62: Overall Received Power vs. Distance: Long Polarization, 2.245GHz, T1. 


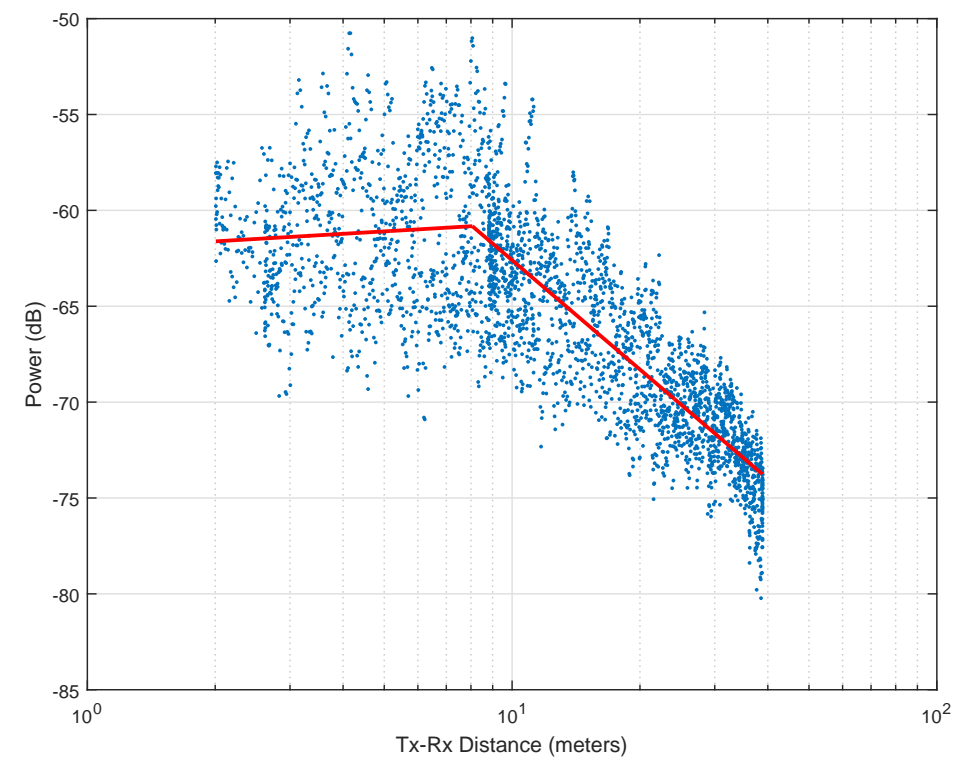

Figure 63: Line of Sight Received Power vs. Distance: Long Polarization, 2.245GHz, T1.

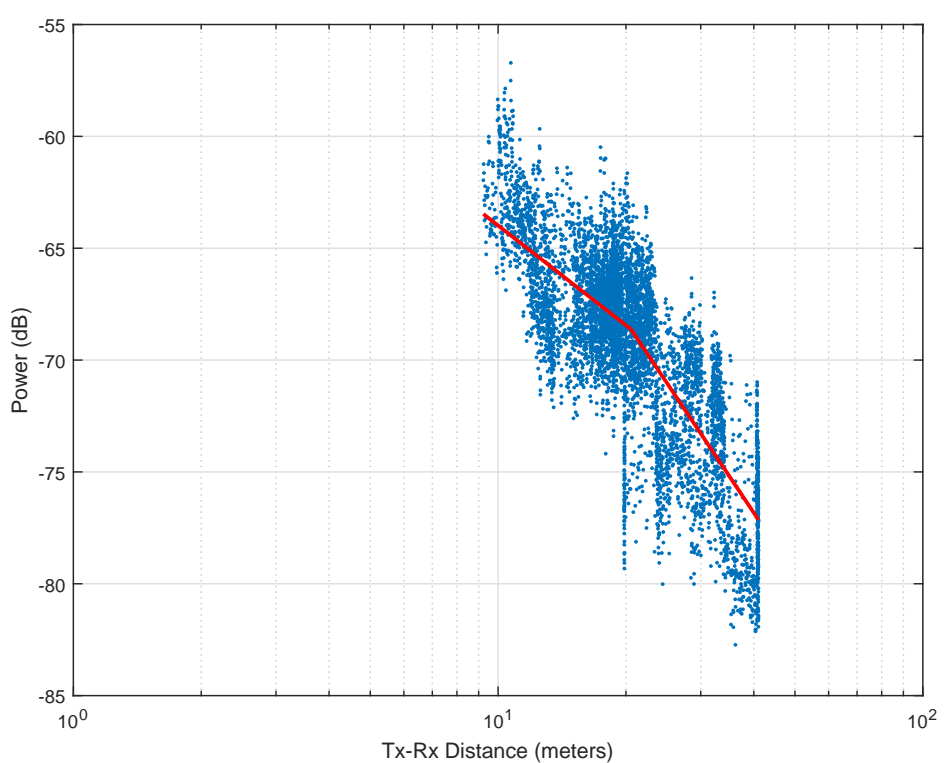

Figure 64: Non Line of Sight Received Power vs. Distance: Long Polarization, 2.245GHz, $\mathrm{T} 1$. 


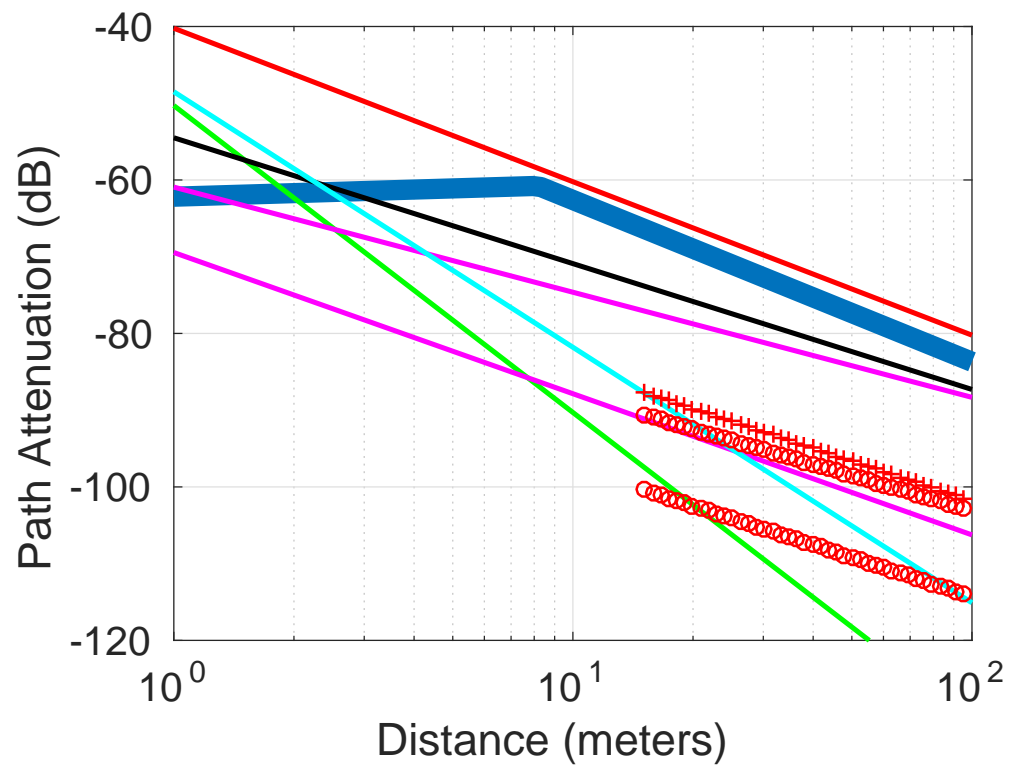

Figure 65: Comparison to other models: Long Polarization, 2.245GHz, T1.

\begin{tabular}{c|c|c|c|c|c|c|c|c}
\hline Type & Frequency & Polarization & $\alpha_{1}$ & $\alpha_{2}$ & $\beta(\mathrm{m})$ & $k_{1}(\mathrm{~dB})$ & $k_{2}(\mathrm{~dB})$ & $\mathrm{MSE}$ \\
\hline \hline Overall & $2.245 \mathrm{GHz}$ & LongPol & -0.15432 & 2.1088 & 8.1815 & -62.1796 & -41.5209 & 9.0812 \\
\hline LOS & $2.245 \mathrm{GHz}$ & LongPol & -0.13092 & 1.8939 & 8.0598 & -62.0107 & -43.6595 & 9.8289 \\
\hline NLOS & $2.245 \mathrm{GHz}$ & LongPol & 1.4813 & 2.8369 & 20.537 & -49.1724 & -31.379 & 8.2535 \\
\hline
\end{tabular}

Table 8: Parameters for Long Polarization, 2.245GHz, T1 


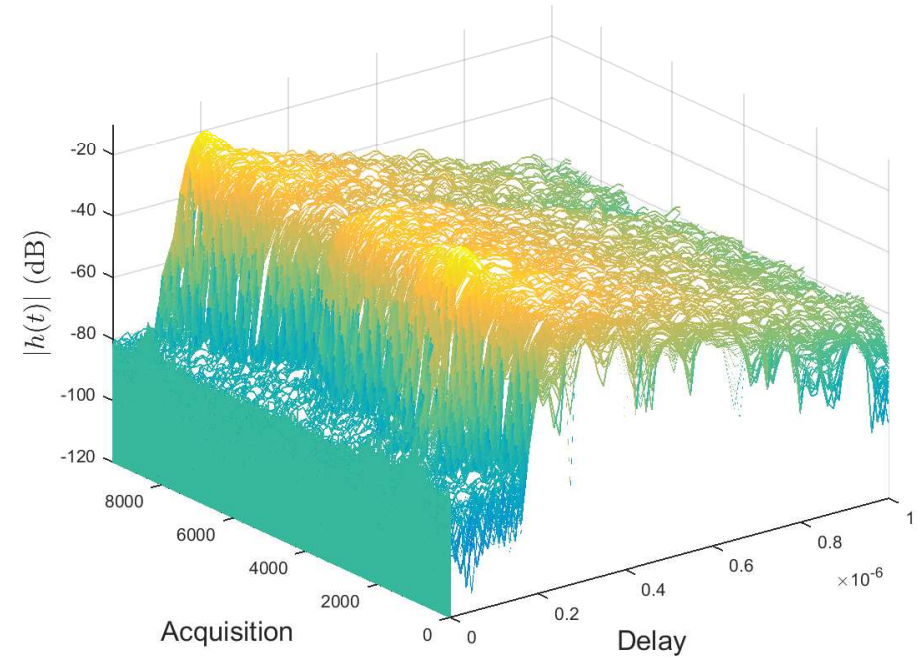

Figure 66: Impulse Responses: Long Polarization, 2.245GHz, T1.

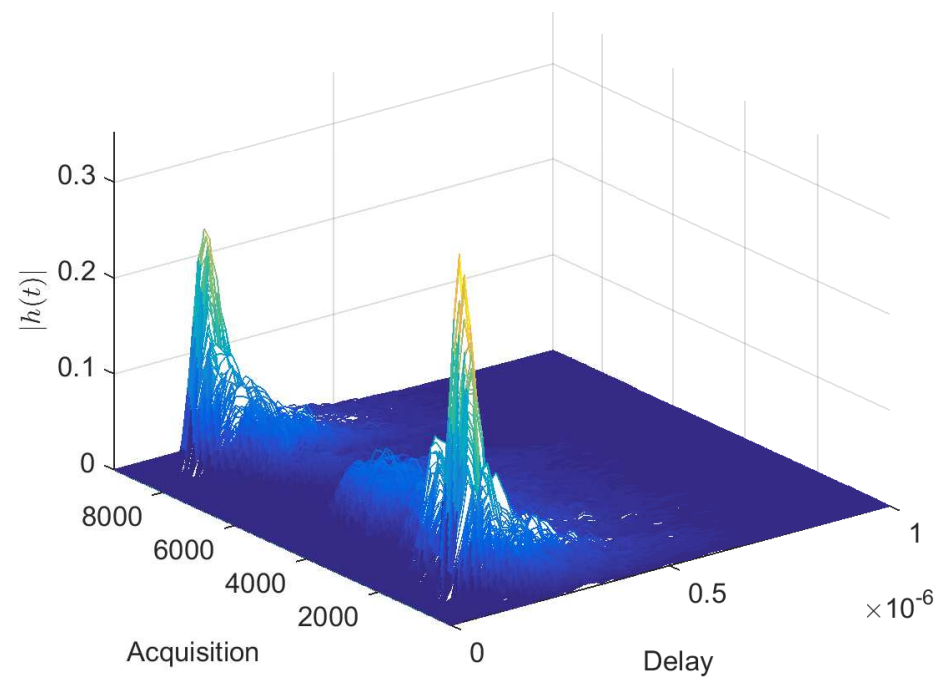

Figure 67: Impulse Responses: Long Polarization, 2.245GHz, T1. 


\section{A.8 Long Polarization, 5GHz, Transmitter 1}

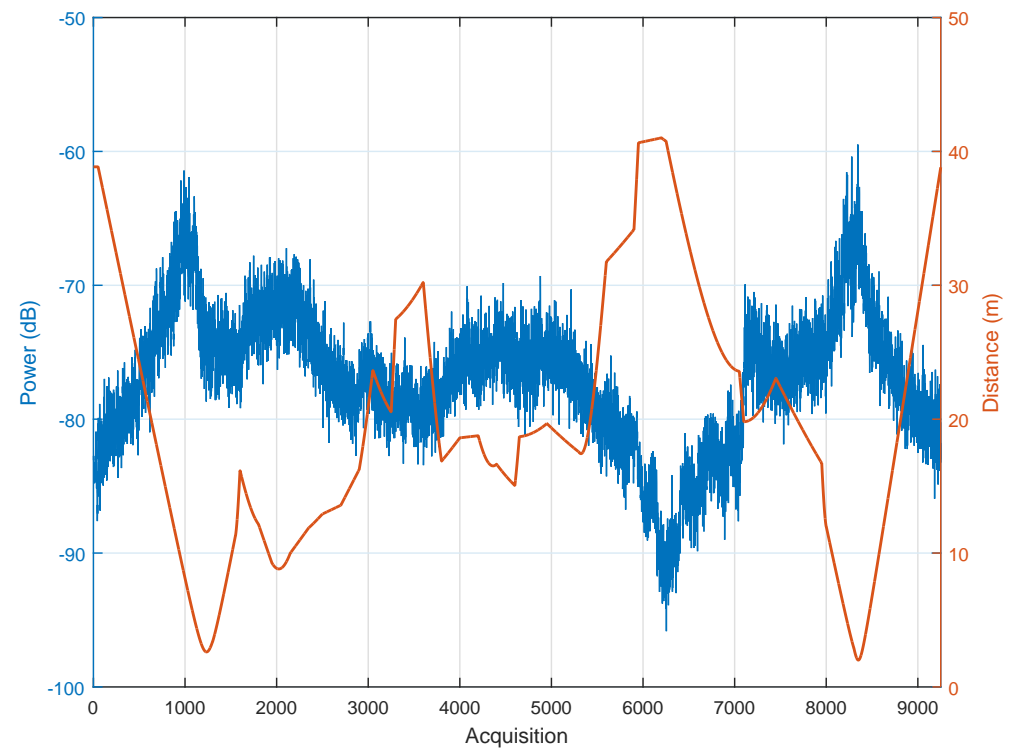

Figure 68: Overall Received Power vs. Acquisition Number and Distance vs. Acquisition Number: Long Polarization, 5.4GHz, T1.

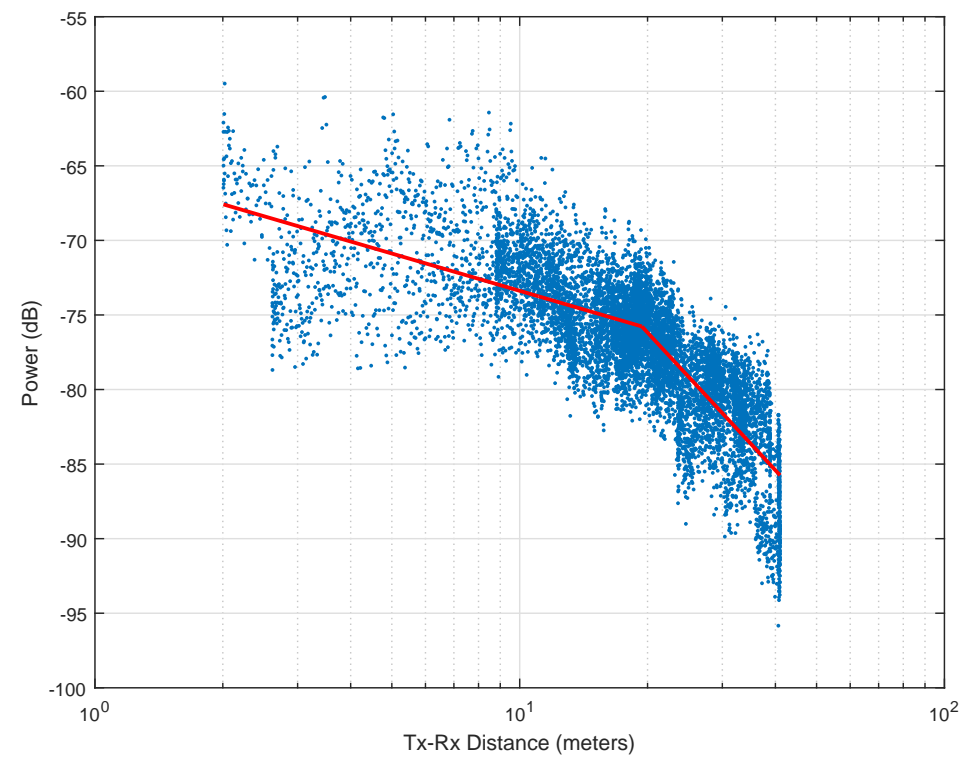

Figure 69: Overall Received Power vs. Distance: Long Polarization, 5.4GHz, T1. 


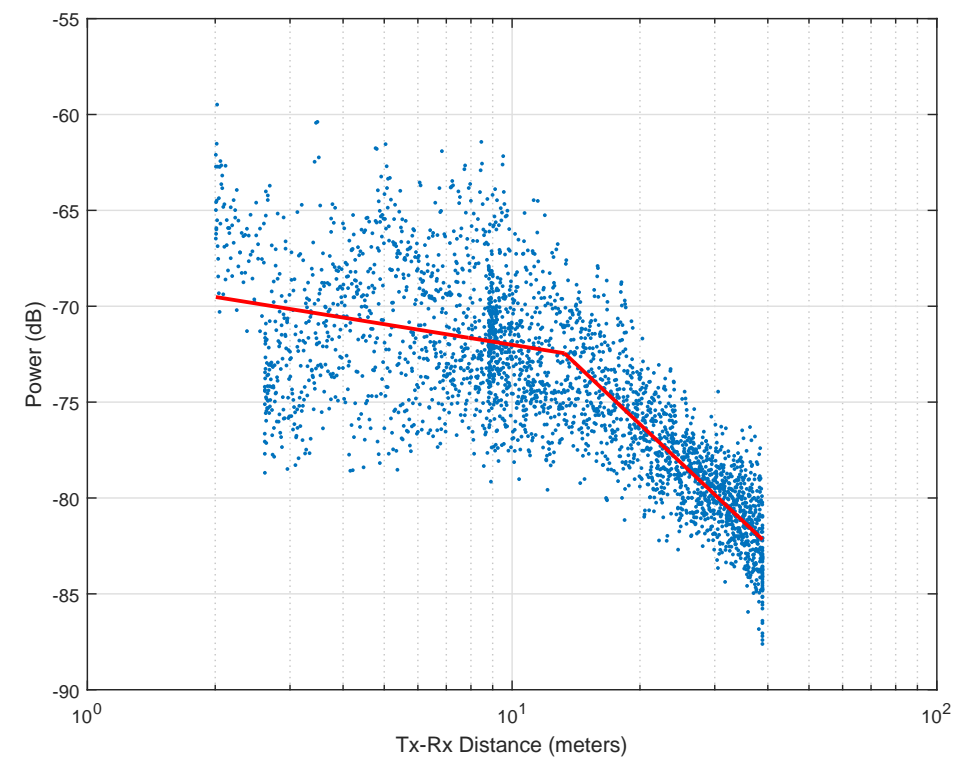

Figure 70: Line of Sight Received Power vs. Distance: Long Polarization, 5.4GHz, T1.

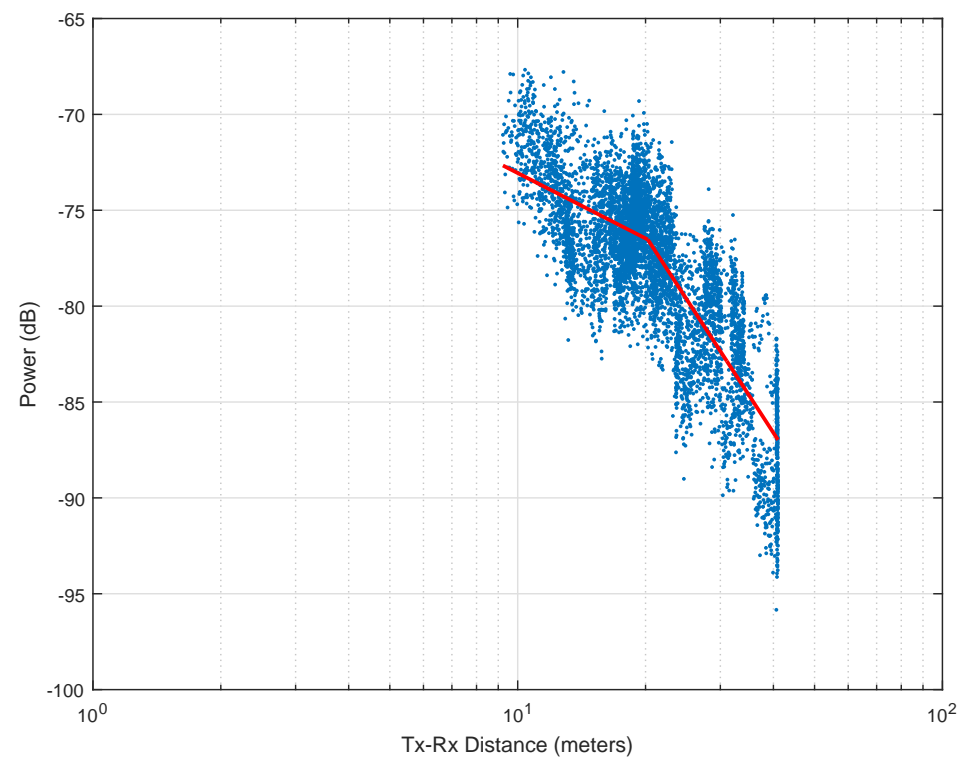

Figure 71: Non Line of Sight Received Power vs. Distance: Long Polarization, 5.4GHz, $\mathrm{T} 1$. 


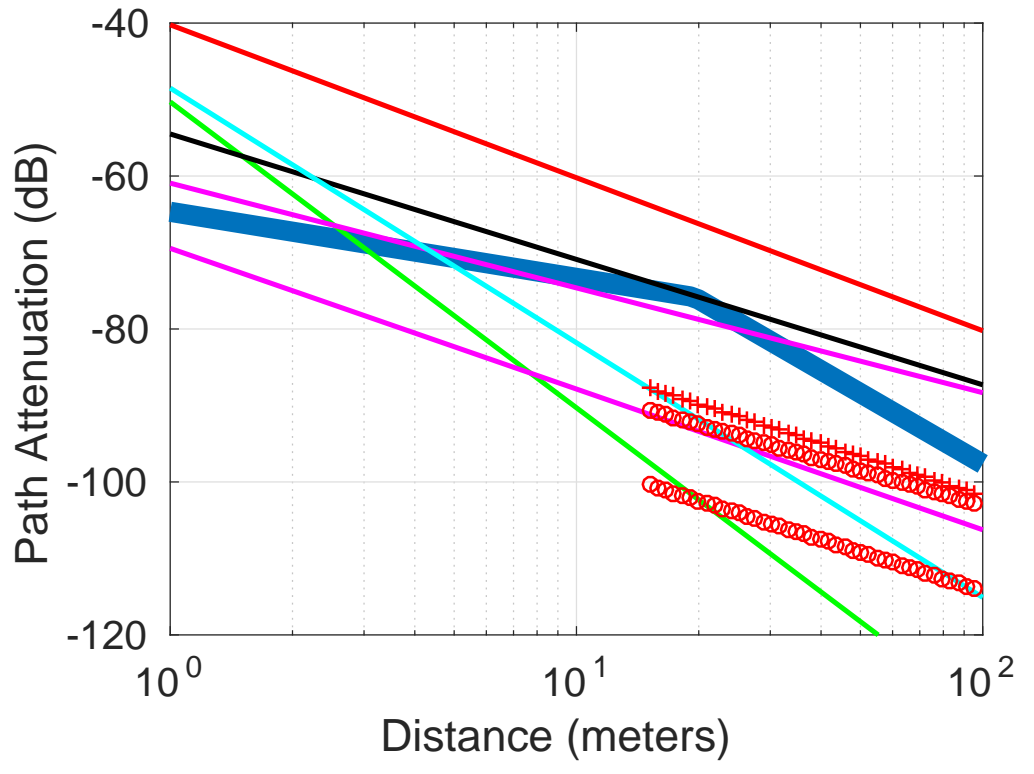

Figure 72: Comparison to other models: Long Polarization, 5.4GHz, T1.

\begin{tabular}{c|c|c|c|c|c|c|c|c}
\hline Type & Frequency & Polarization & $\alpha_{1}$ & $\alpha_{2}$ & $\beta(\mathrm{m})$ & $k_{1}(\mathrm{~dB})$ & $k_{2}(\mathrm{~dB})$ & MSE \\
\hline \hline Overall & $5.4 \mathrm{GHz}$ & LongPol & 0.86433 & 3.0744 & 19.5126 & -64.6758 & -36.1586 & 9.101 \\
\hline LOS & $5.4 \mathrm{GHz}$ & LongPol & 0.3571 & 2.0845 & 13.2858 & -68.4398 & -49.0348 & 9.2727 \\
\hline NLOS & $5.4 \mathrm{GHz}$ & LongPol & 1.1325 & 3.4085 & 20.2497 & -61.7334 & -31.9992 & 8.2512 \\
\hline
\end{tabular}

Table 9: Parameters for Long Polarization, 5GHz, T1 


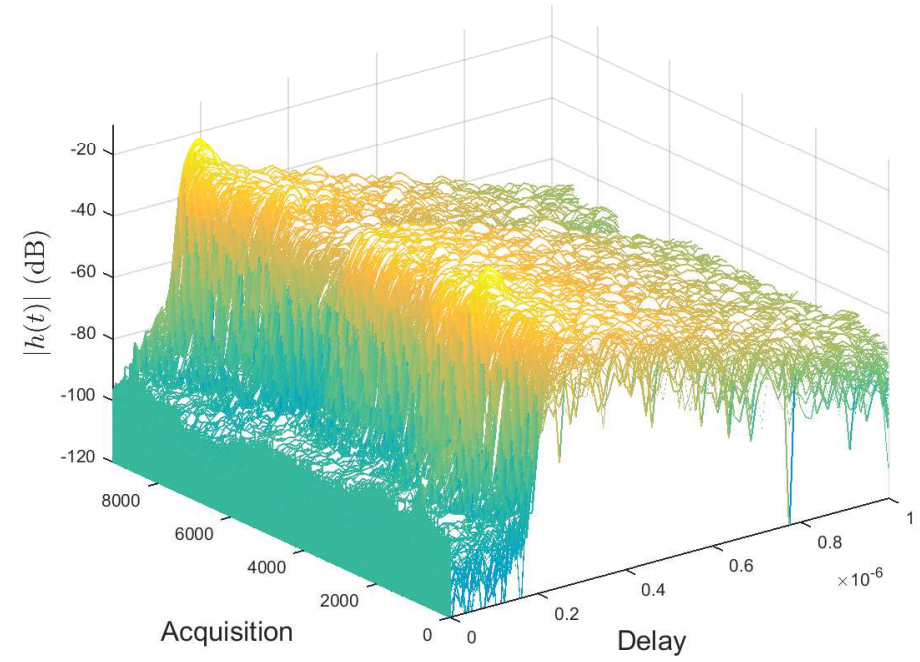

Figure 73: Impulse Responses: Long Polarization, 5.4GHz, T1.

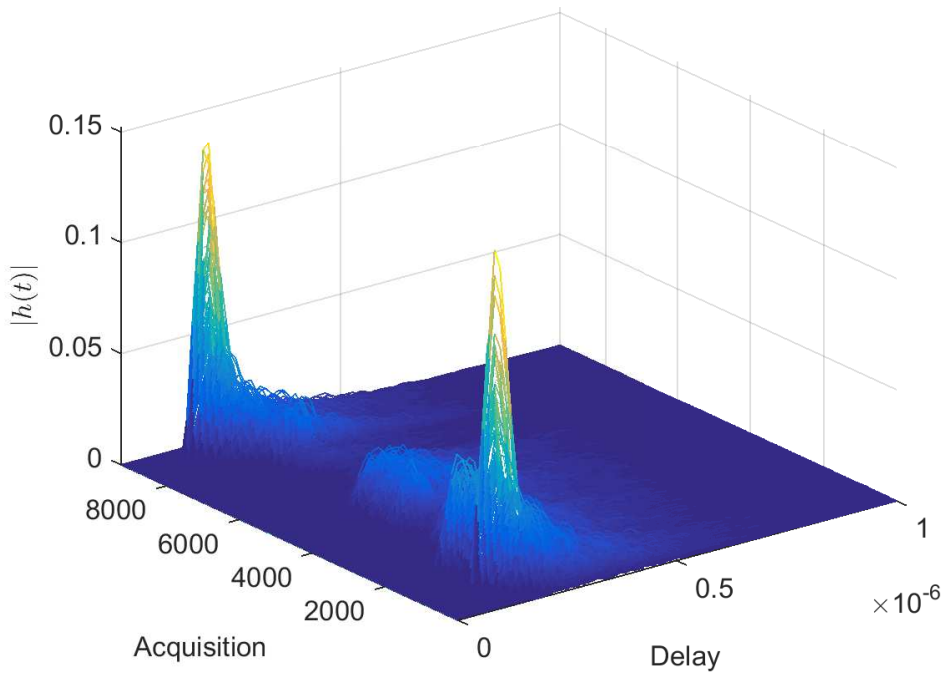

Figure 74: Impulse Responses: Long Polarization, 5.4GHz, T1. 


\section{A.9 Long Polarization, 2.245GHz, Transmitter 2, High}

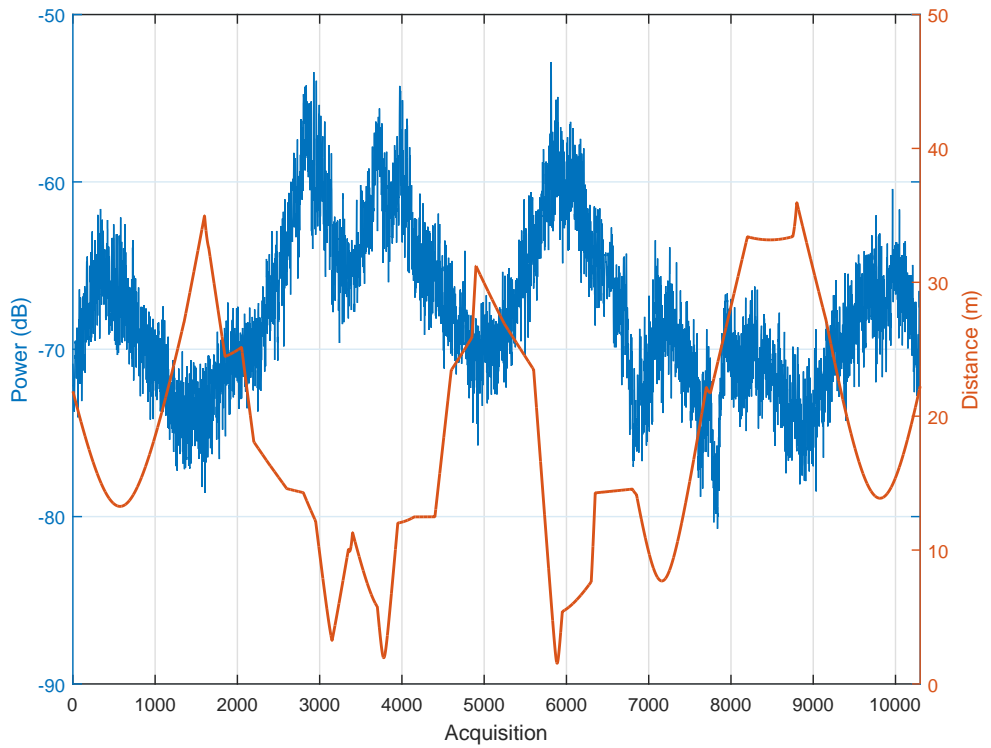

Figure 75: Overall Received Power vs. Acquisition Number and Distance vs. Acquisition Number: Long Polarization, 2.245GHz, T2, High.

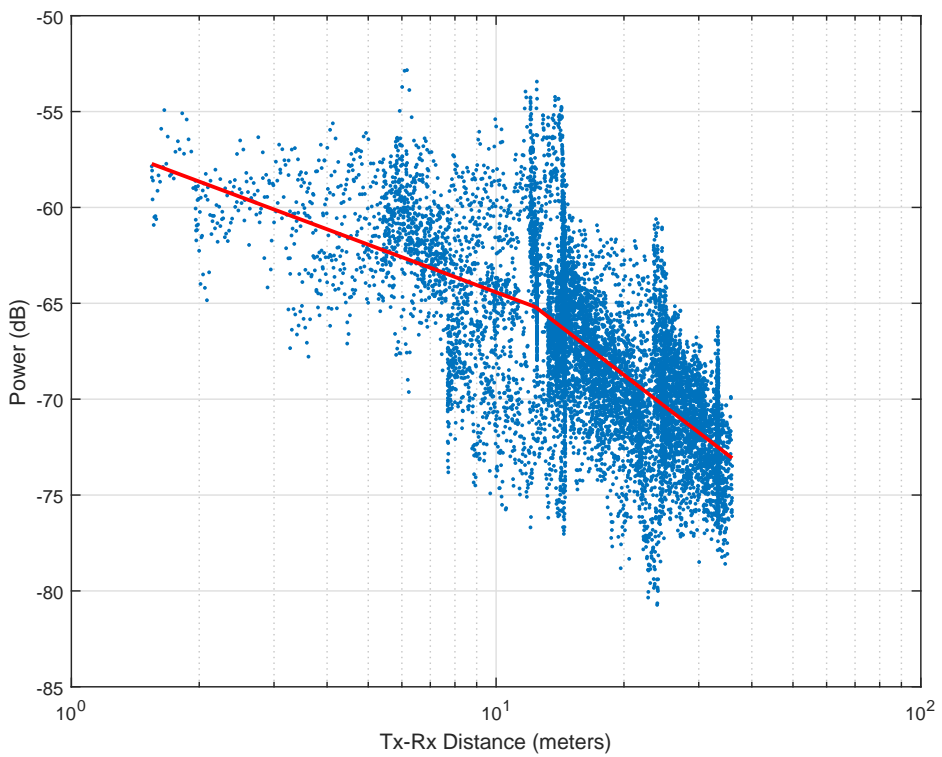

Figure 76: Overall Received Power vs. Distance: Long Polarization, 2.245GHz, T2, High. 


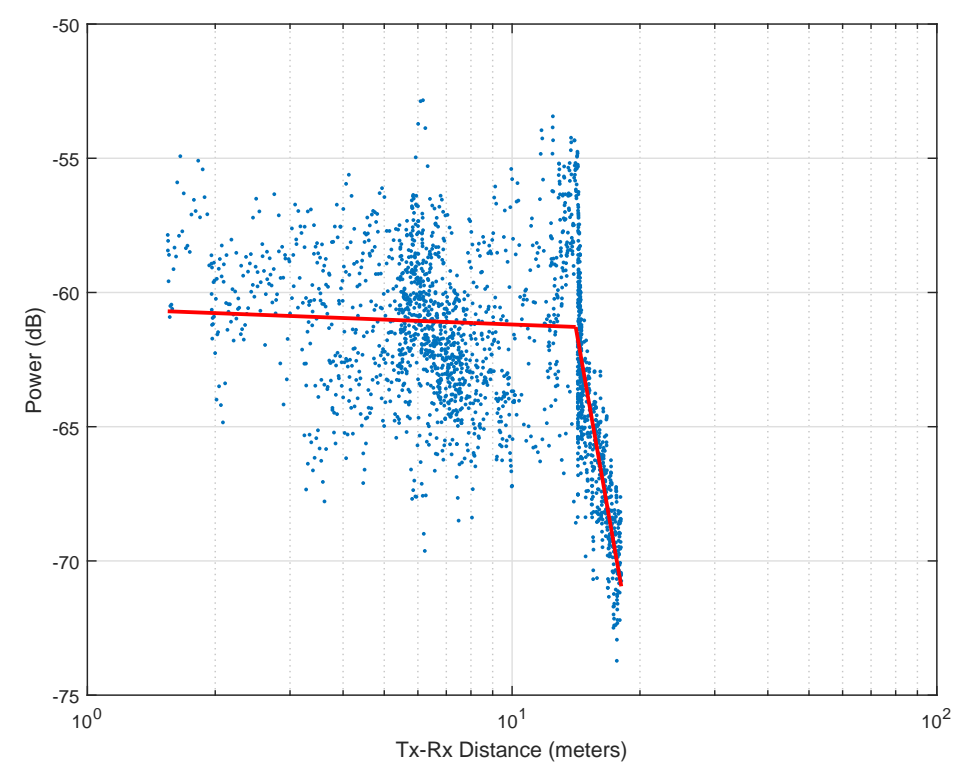

Figure 77: Line of Sight Received Power vs. Distance: Long Polarization, 2.245GHz, T2, High.

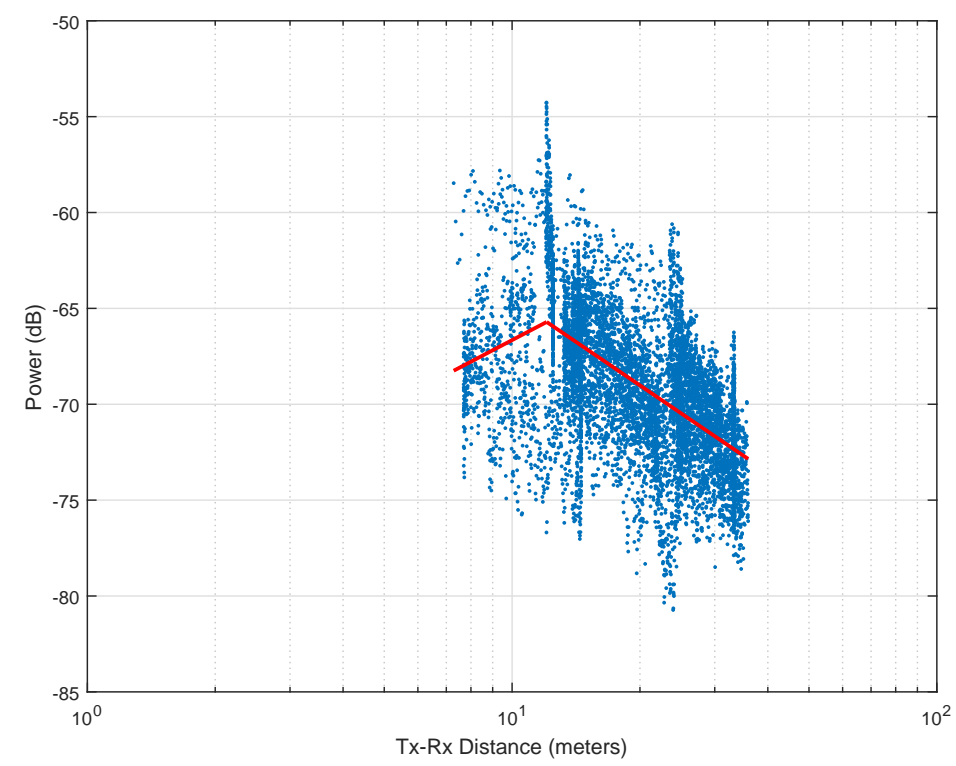

Figure 78: Non Line of Sight Received Power vs. Distance: Long Polarization, 2.245GHz, T2, High. 


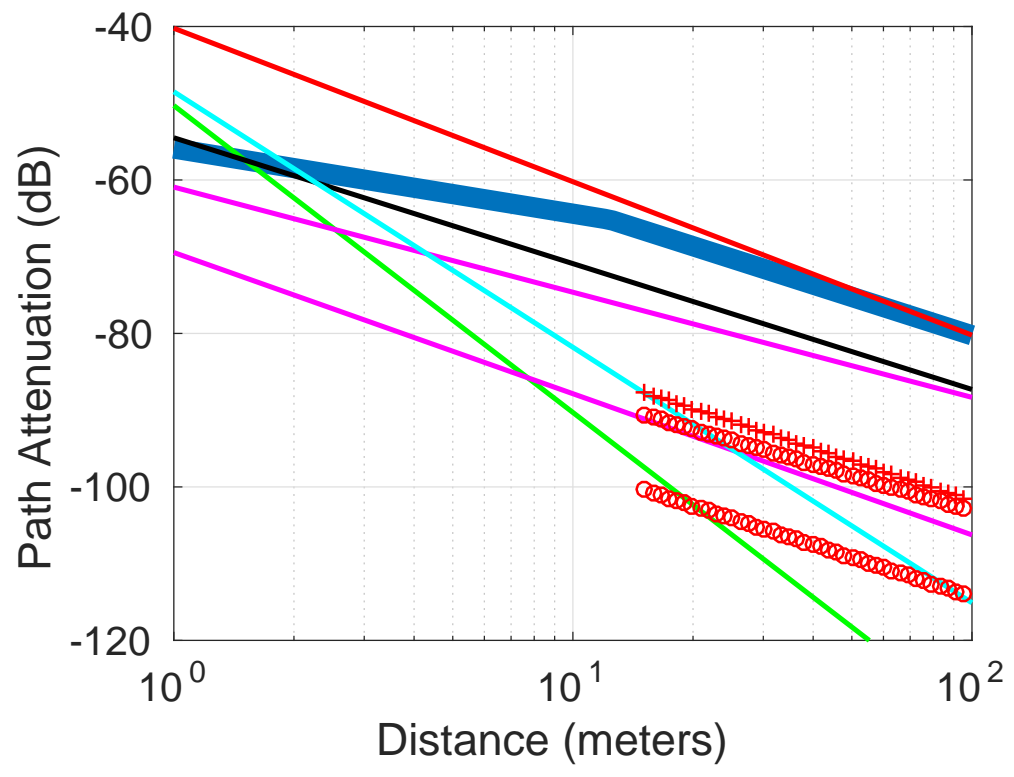

Figure 79: Comparison to other models: Long Polarization, 2.245GHz, T2, High.

\begin{tabular}{c|c|c|c|c|c|c|c|c}
\hline Type & Frequency & Polarization & $\alpha_{1}$ & $\alpha_{2}$ & $\beta(\mathrm{m})$ & $k_{1}(\mathrm{~dB})$ & $k_{2}(\mathrm{~dB})$ & MSE \\
\hline \hline Overall & $2.245 \mathrm{GHz}$ & LongPol High & 0.84922 & 1.6614 & 12.4132 & -55.9006 & -47.0167 & 10.8449 \\
\hline LOS & $2.245 \mathrm{GHz}$ & LongPol High & 0.060714 & 9.0037 & 14.1156 & -60.5855 & 42.2322 & 8.2734 \\
\hline NLOS & $2.245 \mathrm{GHz}$ & LongPol High & -1.1611 & 1.5052 & 12.0615 & -78.2653 & -49.4317 & 9.8642 \\
\hline
\end{tabular}

Table 10: Parameters for Long Polarization, 2.245GHz, T2, High 


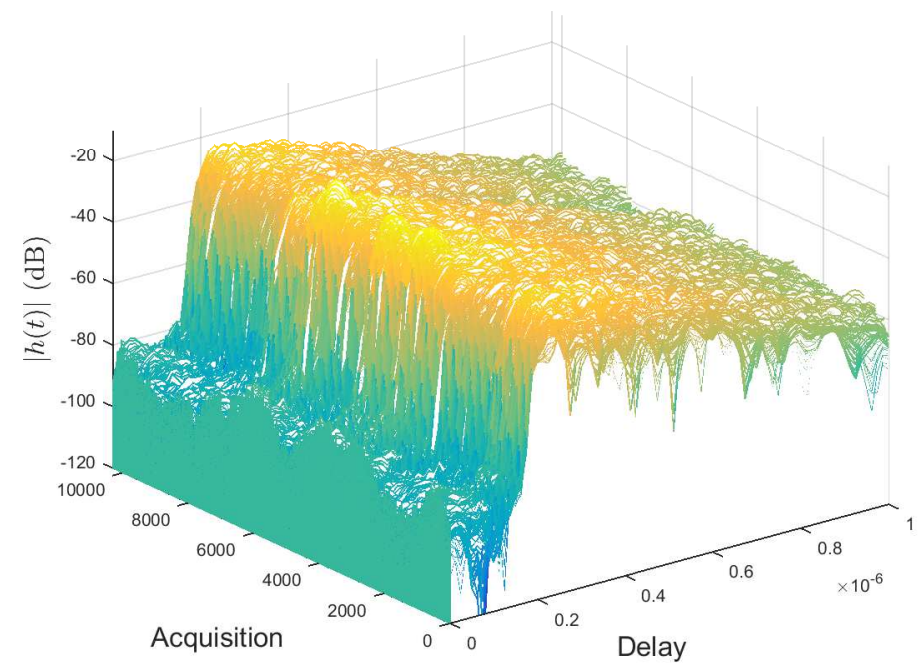

Figure 80: Impulse Responses: Long Polarization, 2.245GHz, T2, High.

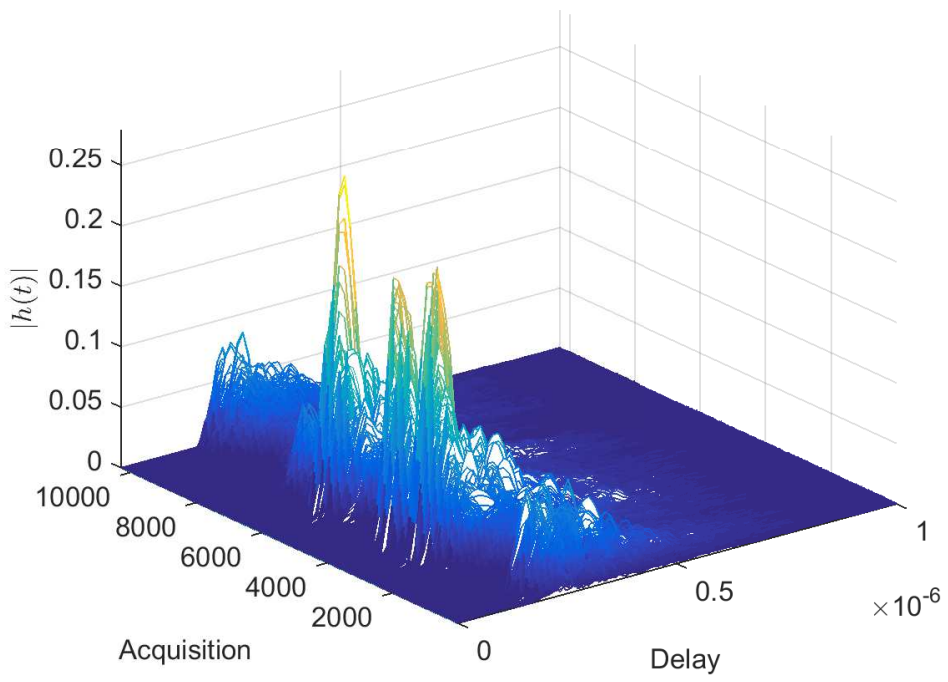

Figure 81: Impulse Responses: Long Polarization, 2.245GHz, T2, High. 


\section{A.10 Long Polarization, 2.245GHz, Transmitter 2, Low}

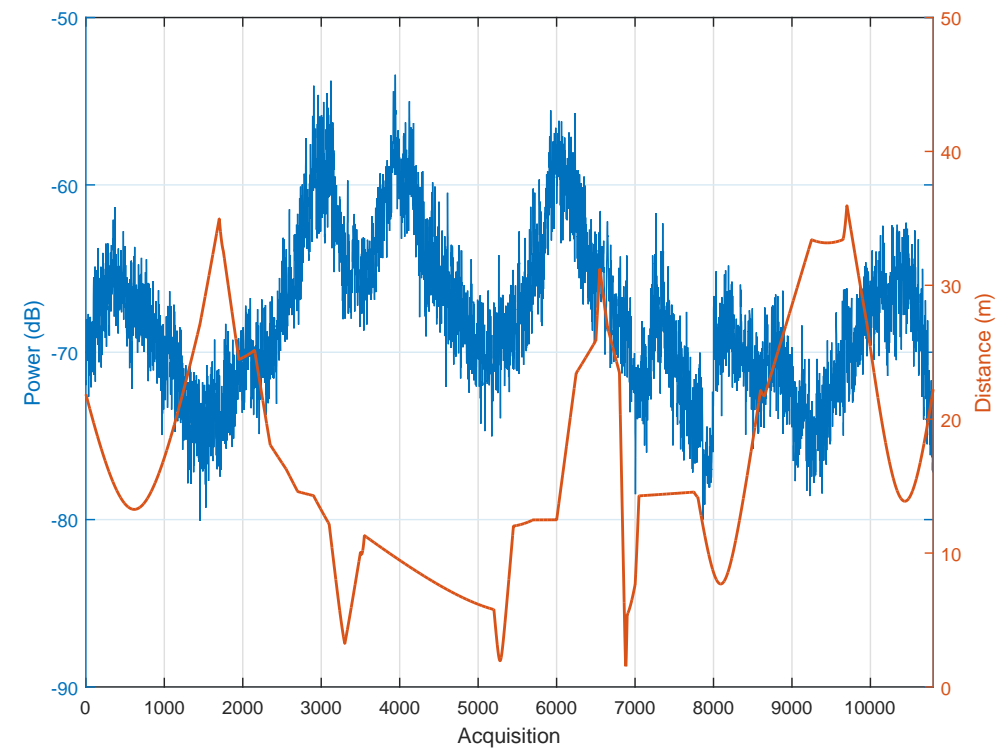

Figure 82: Overall Received Power vs. Acquisition Number and Distance vs. Acquisition Number: Long Polarization, 2.245GHz, T2, Low.

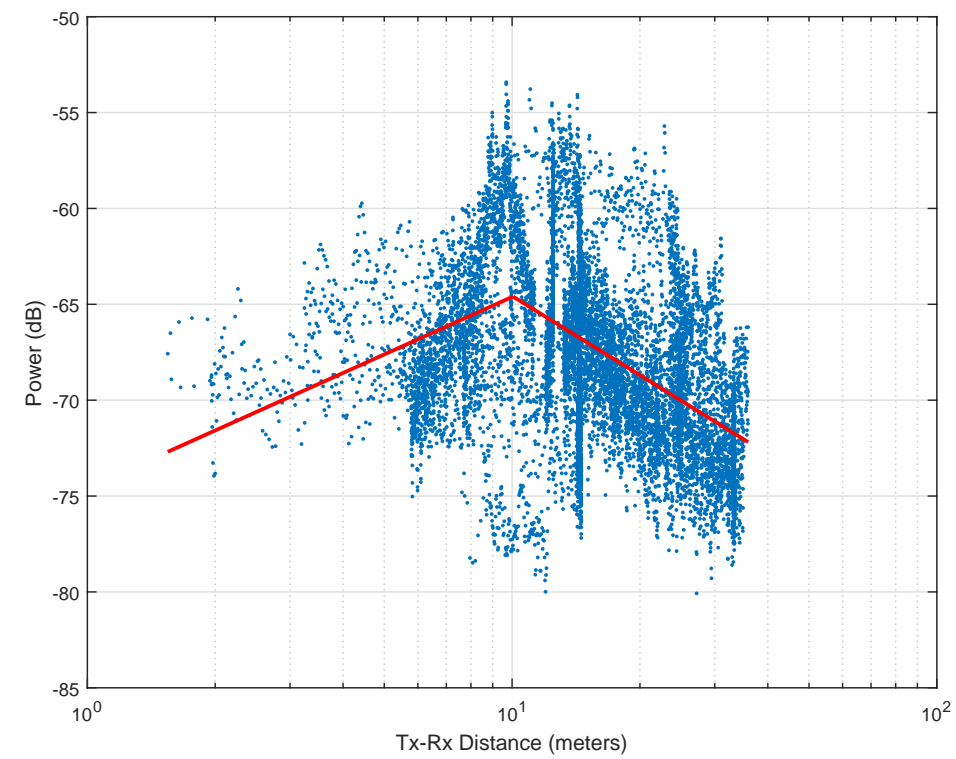

Figure 83: Overall Received Power vs. Distance: Long Polarization, 2.245GHz, T2, Low. 


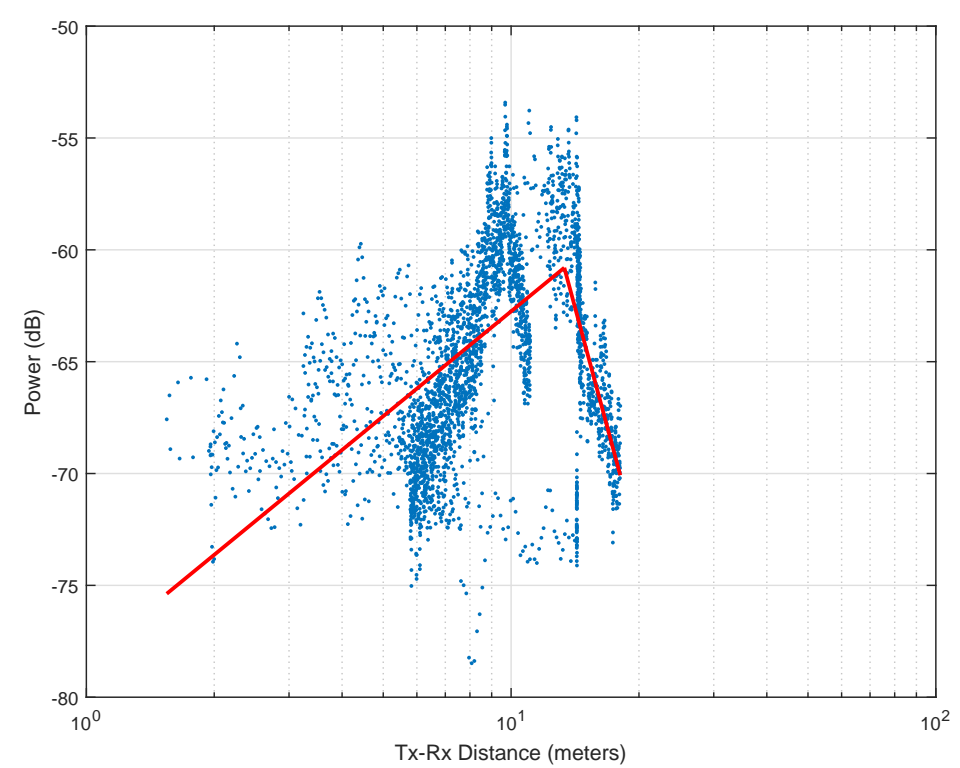

Figure 84: Line of Sight Received Power vs. Distance: Long Polarization, 2.245GHz, T2, Low.

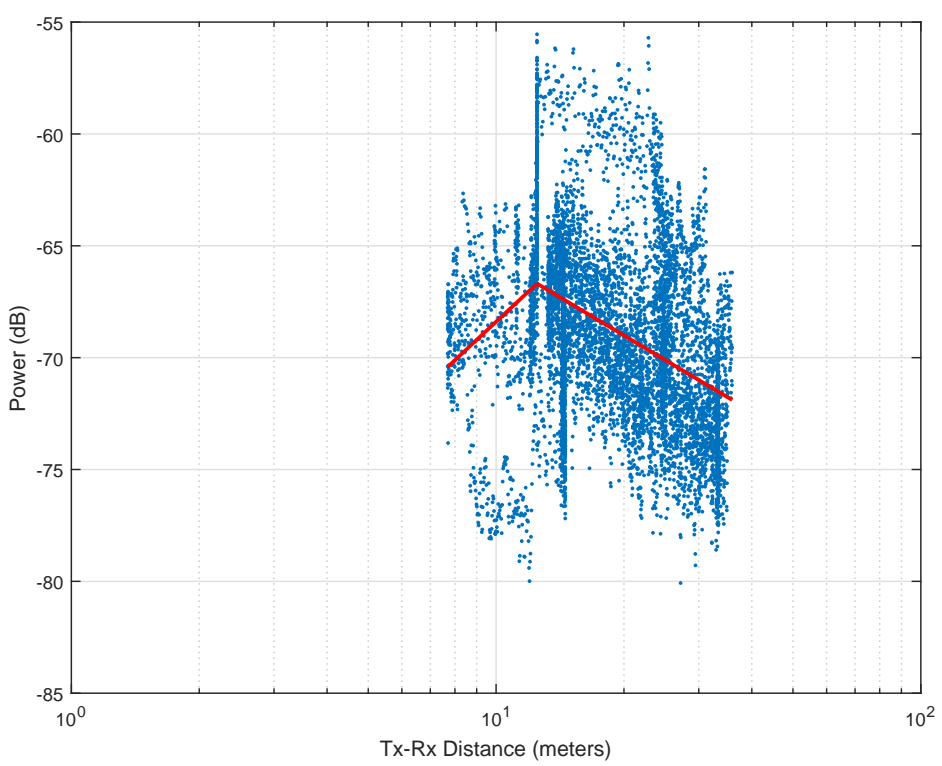

Figure 85: Non Line of Sight Received Power vs. Distance: Long Polarization, 2.245GHz, T2, Low. 


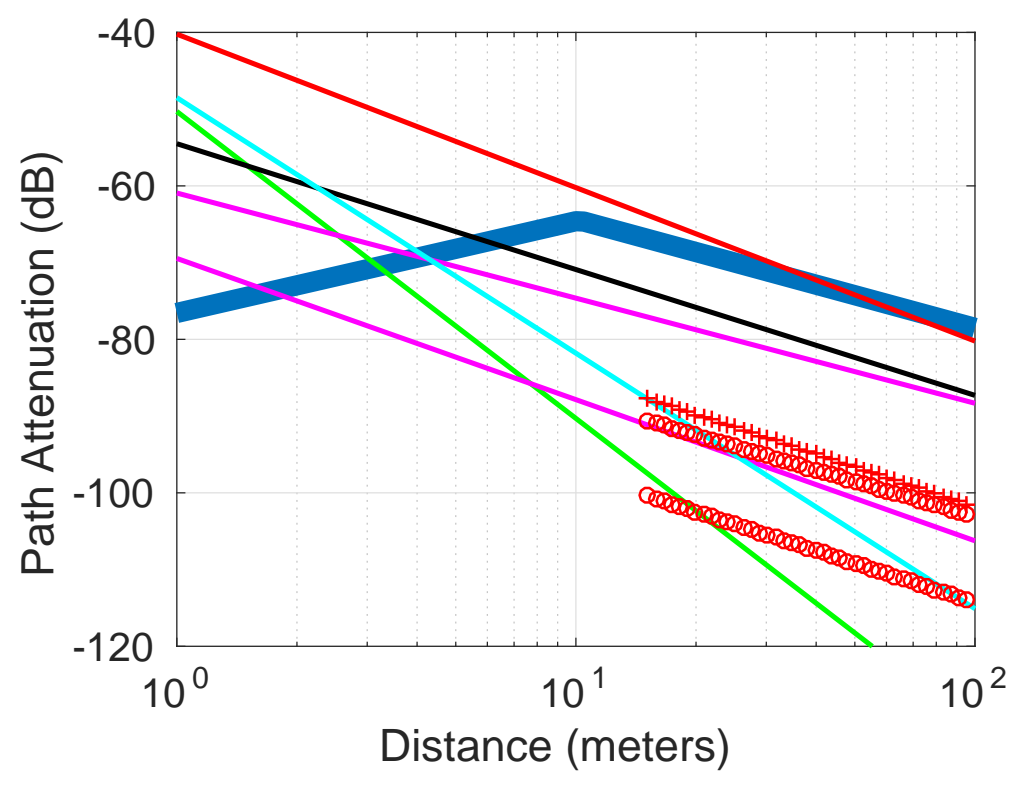

Figure 86: Comparison to other models: Long Polarization, $2.245 \mathrm{GHz}$, T2, Low.

\begin{tabular}{c|c|c|c|c|c|c|c|c}
\hline Type & Frequency & Polarization & $\alpha_{1}$ & $\alpha_{2}$ & $\beta(\mathrm{m})$ & $k_{1}(\mathrm{~dB})$ & $k_{2}(\mathrm{~dB})$ & MSE \\
\hline \hline Overall & $2.245 \mathrm{GHz}$ & LongPol Low & -1.1956 & 1.4134 & 10.2097 & -76.5594 & -50.2345 & 12.093 \\
\hline LOS & $2.245 \mathrm{GHz}$ & LongPol Low & -1.557 & 7.0381 & 13.3428 & -78.3206 & 18.3958 & 11.2471 \\
\hline NLOS & $2.245 \mathrm{GHz}$ & LongPol Low & -1.7714 & 1.1321 & 12.4823 & -86.108 & -54.2775 & 11.4781 \\
\hline
\end{tabular}

Table 11: Parameters for Long Polarization, 2.245GHz, T2, Low 


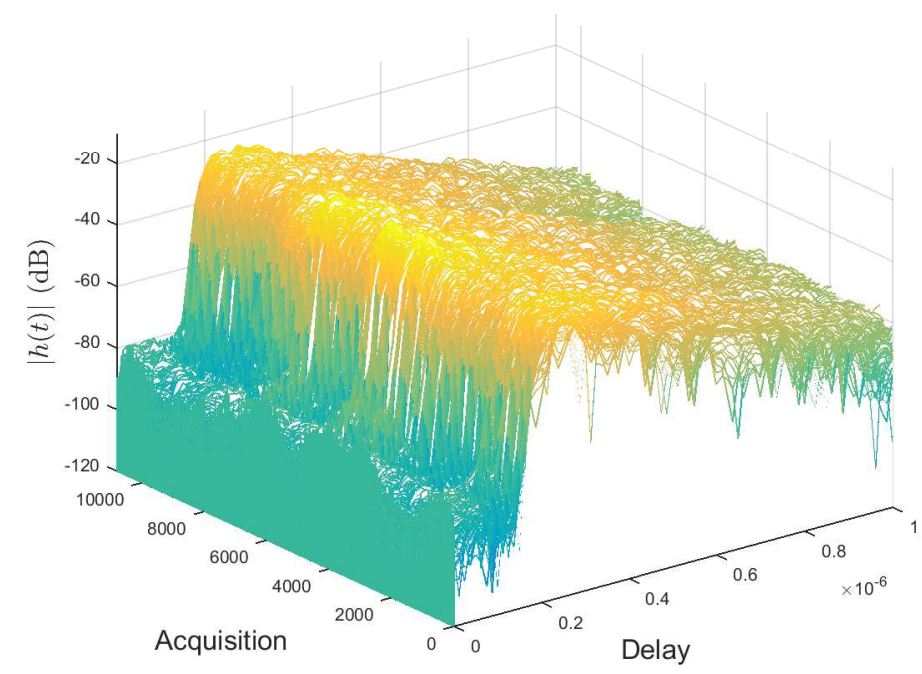

Figure 87: Impulse Responses: Long Polarization, 2.245GHz, T2, Low.

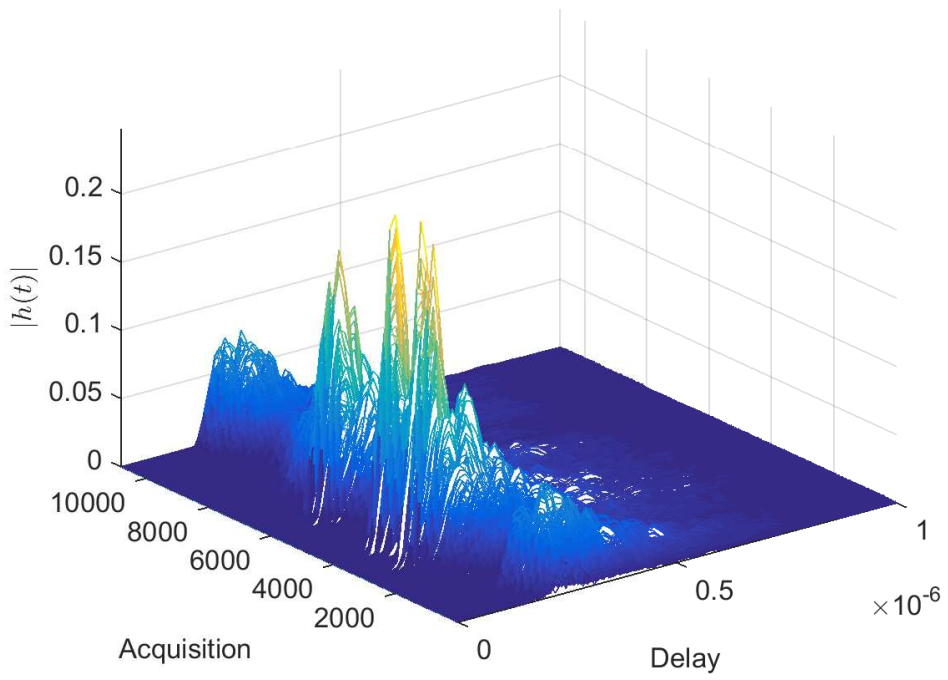

Figure 88: Impulse Responses: Long Polarization, 2.245GHz, T2, Low. 


\section{A.11 Long Polarization, 5GHz, Transmitter 2, High}

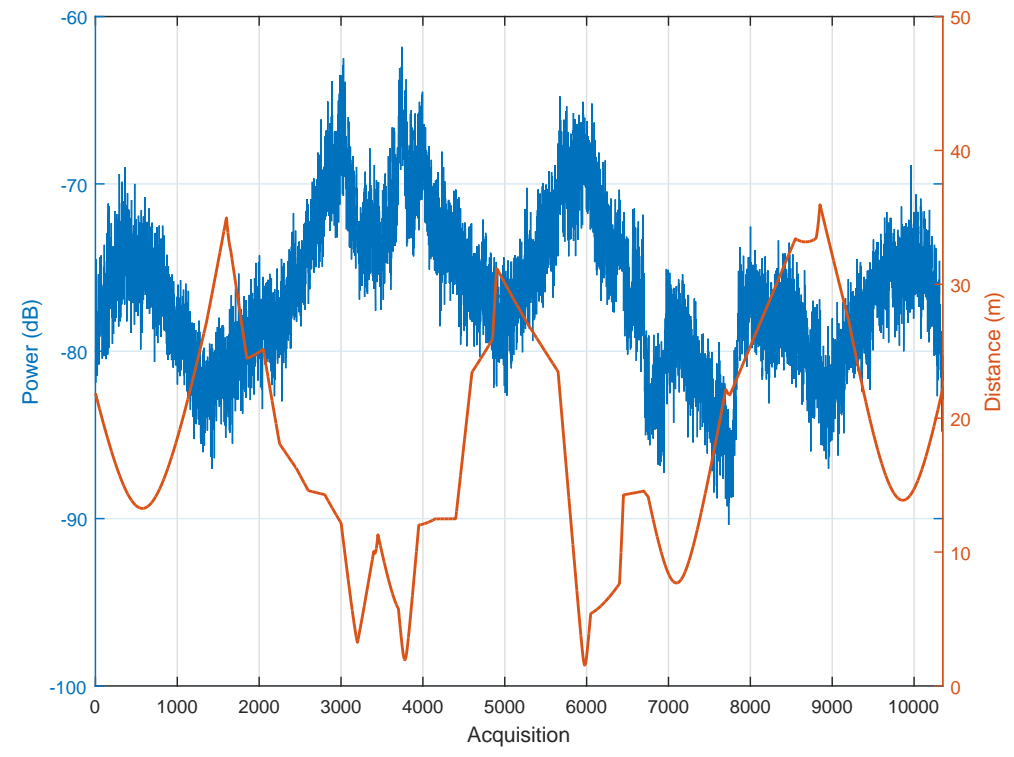

Figure 89: Overall Received Power vs. Acquisition Number and Distance vs. Acquisition Number: Long Polarization, 5.4GHz, T2, High.

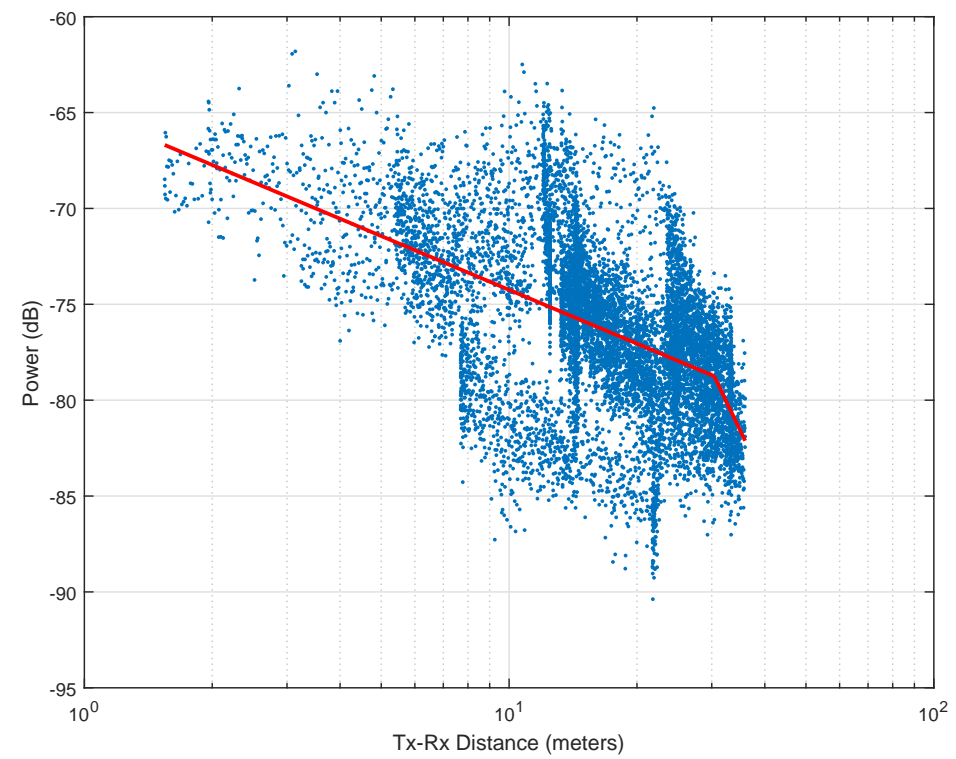

Figure 90: Overall Received Power vs. Distance: Long Polarization, 5.4GHz, T2, High. 


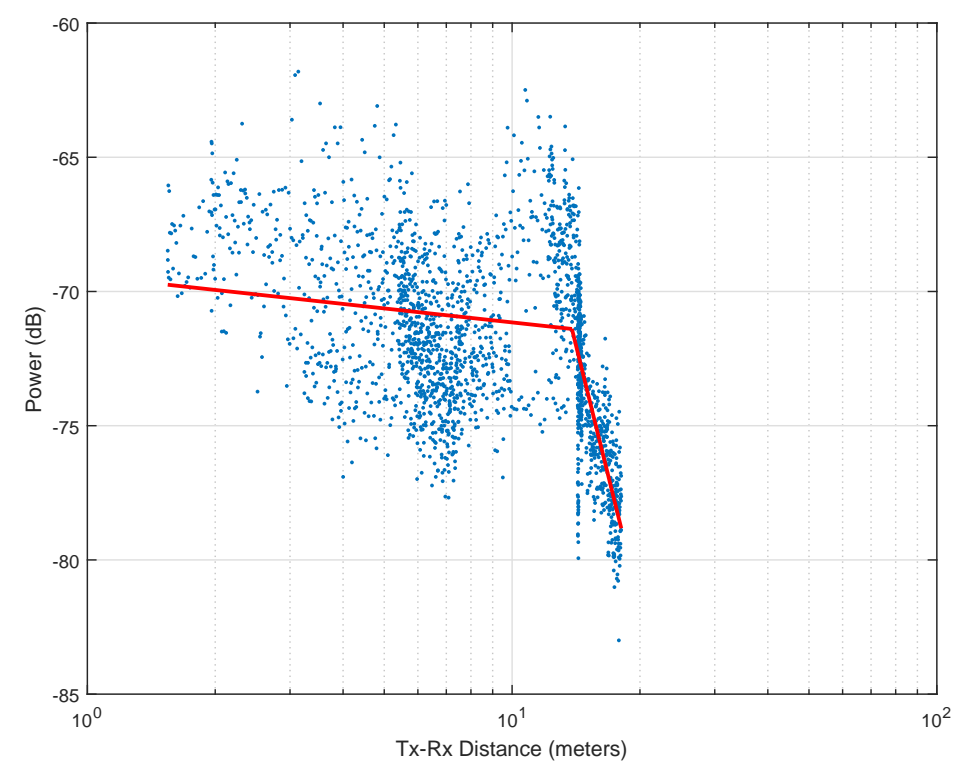

Figure 91: Line of Sight Received Power vs. Distance: Long Polarization, 5.4GHz, T2, High.

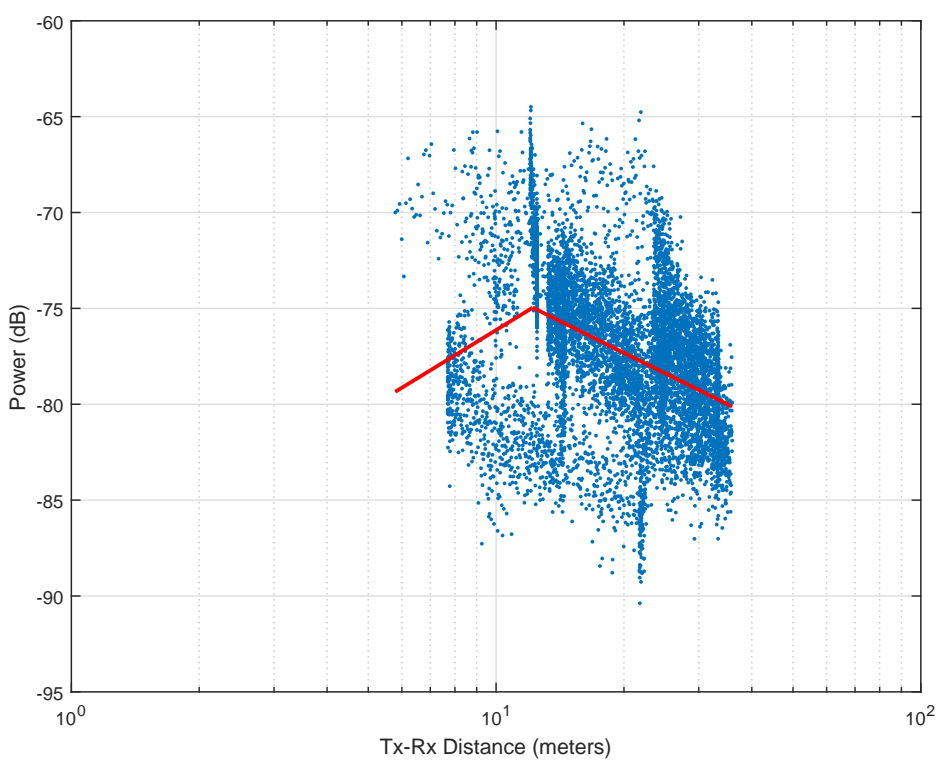

Figure 92: Non Line of Sight Received Power vs. Distance: Long Polarization, 5.4GHz, T2, High. 


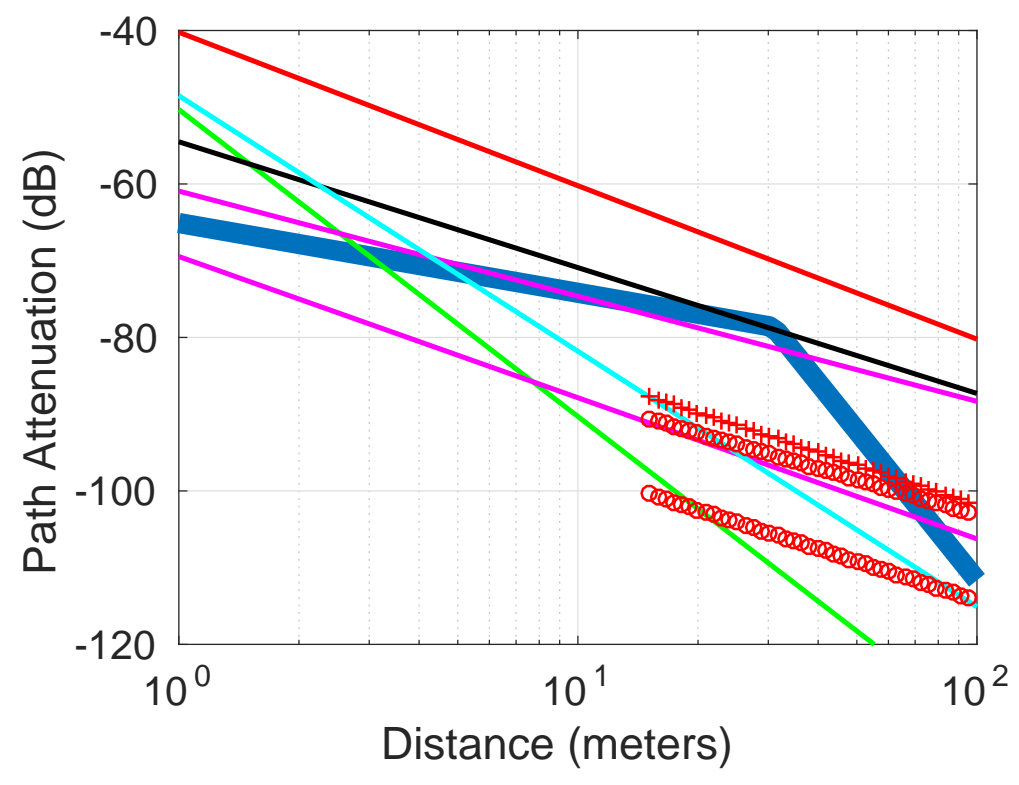

Figure 93: Comparison to other models: Long Polarization, 5.4GHz, T2, High.

\begin{tabular}{c|c|c|c|c|c|c|c|c}
\hline Type & Frequency & Polarization & $\alpha_{1}$ & $\alpha_{2}$ & $\beta(\mathrm{m})$ & $k_{1}(\mathrm{~dB})$ & $k_{2}(\mathrm{~dB})$ & MSE \\
\hline \hline Overall & $5.4 \mathrm{GHz}$ & LongPol High & 0.90942 & 6.478 & 30.9429 & -65.0881 & 17.9146 & 11.5051 \\
\hline LOS & $5.4 \mathrm{GHz}$ & LongPol High & 0.17308 & 6.3922 & 13.8303 & -69.4213 & 1.5281 & 8.4983 \\
\hline NLOS & $5.4 \mathrm{GHz}$ & LongPol High & -1.3559 & 1.0987 & 12.1978 & -89.6896 & -63.0264 & 11.156 \\
\hline
\end{tabular}

Table 12: Parameters for Long Polarization, 5GHz, T2, High 


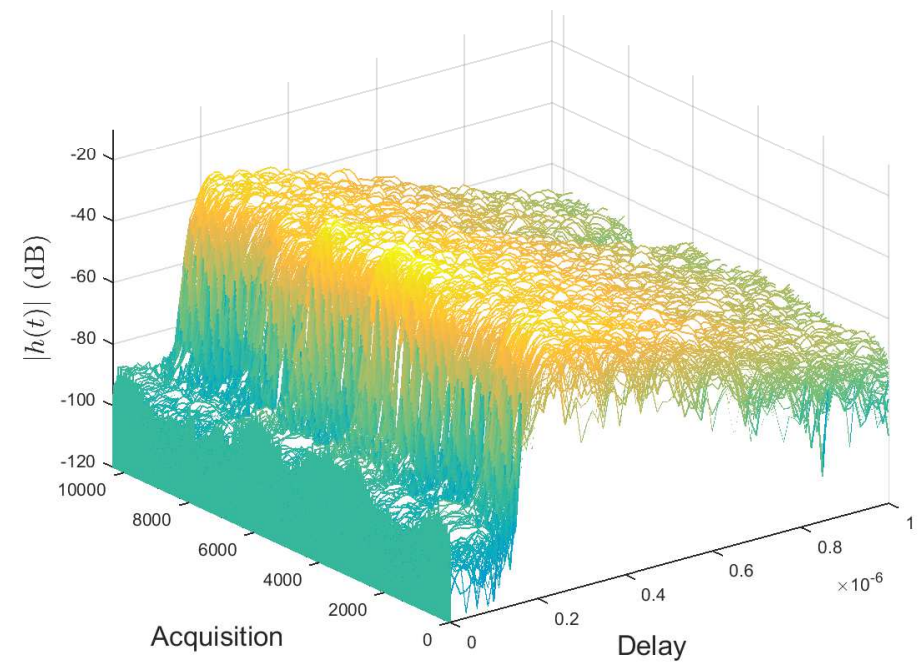

Figure 94: Impulse Responses: Long Polarization, 5.4GHz, T2, High.

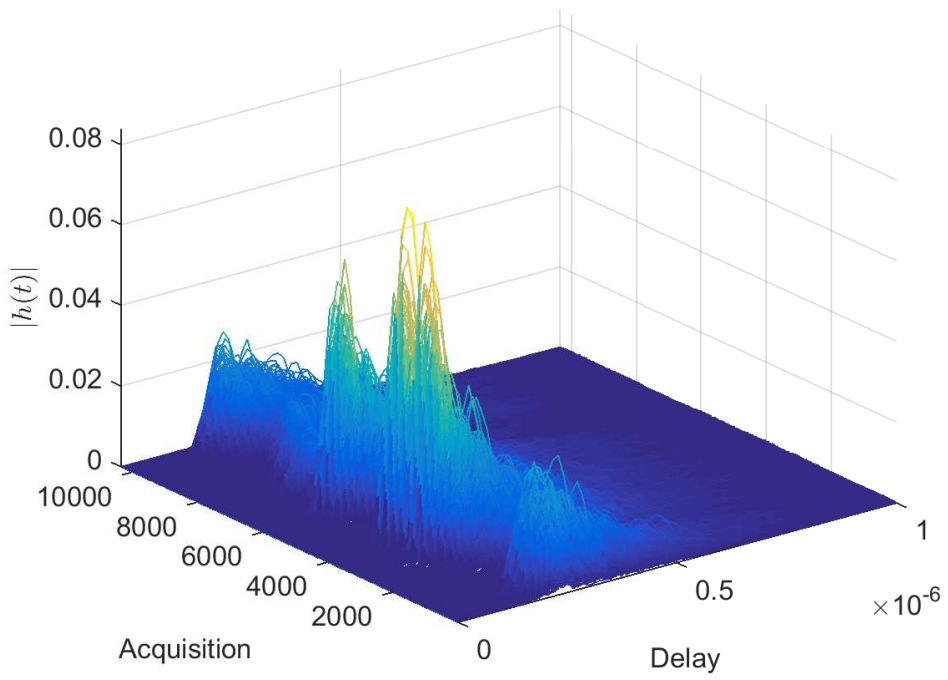

Figure 95: Impulse Responses: Long Polarization, 5.4GHz, T2, High. 


\section{A.12 Long Polarization, 5GHz, Transmitter 2, Low}

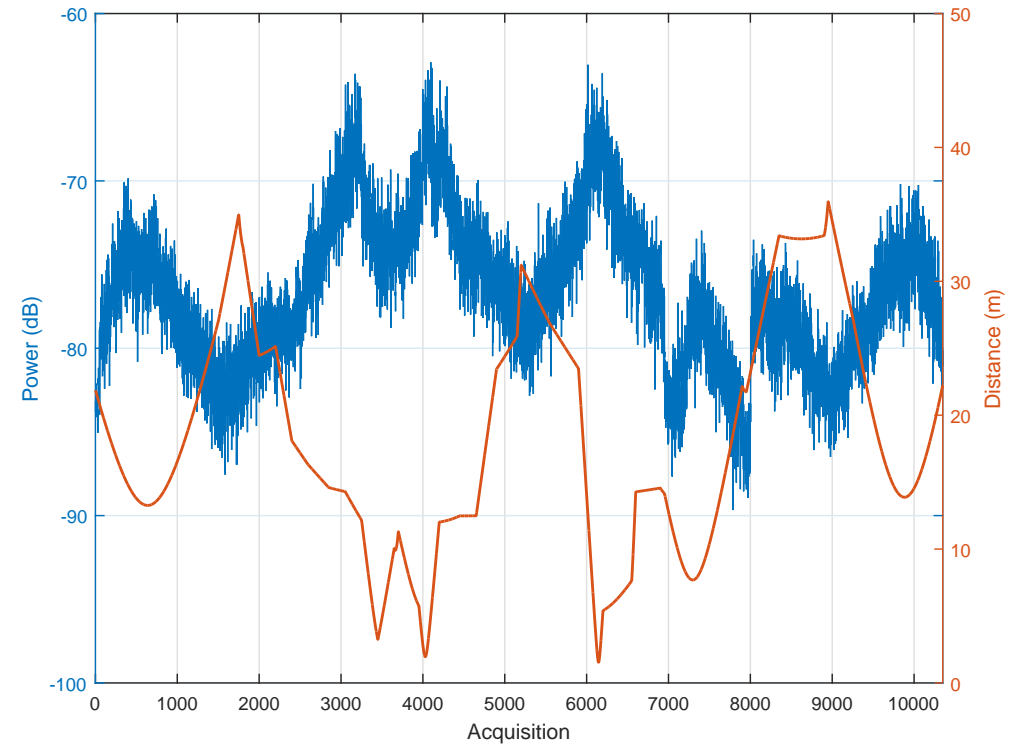

Figure 96: Overall Received Power vs. Acquisition Number and Distance vs. Acquisition Number: Long Polarization, 5.4GHz, T2, Low.

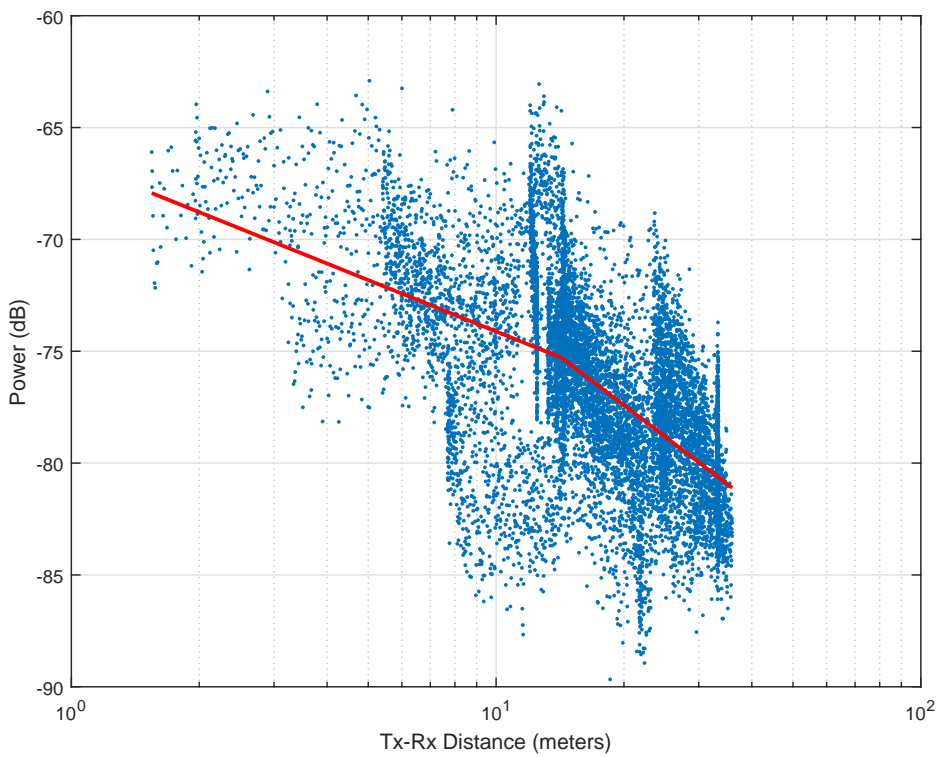

Figure 97: Overall Received Power vs. Distance: Long Polarization, 5.4GHz, T2, Low. 


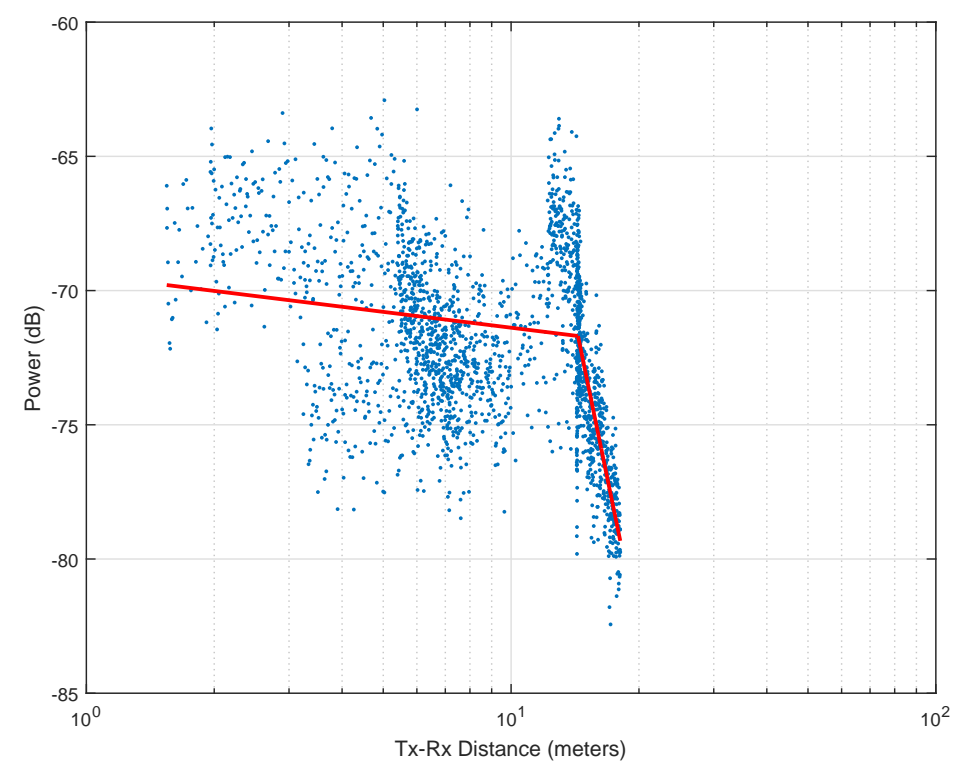

Figure 98: Line of Sight Received Power vs. Distance: Long Polarization, 5.4GHz, T2, Low.

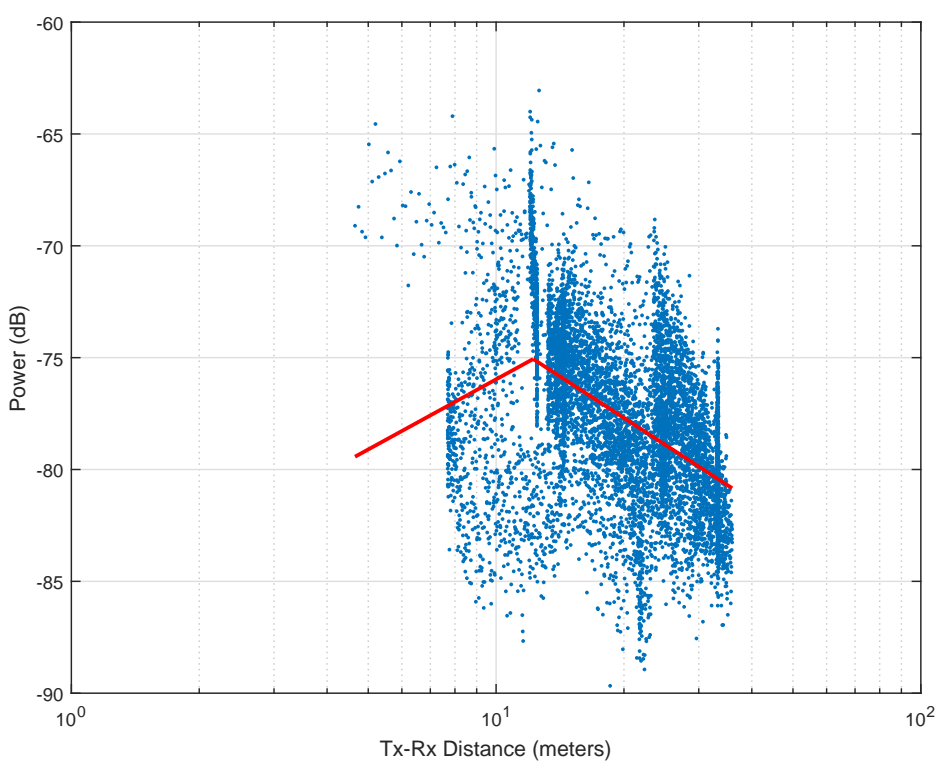

Figure 99: Non Line of Sight Received Power vs. Distance: Long Polarization, 5.4GHz, T2, Low. 


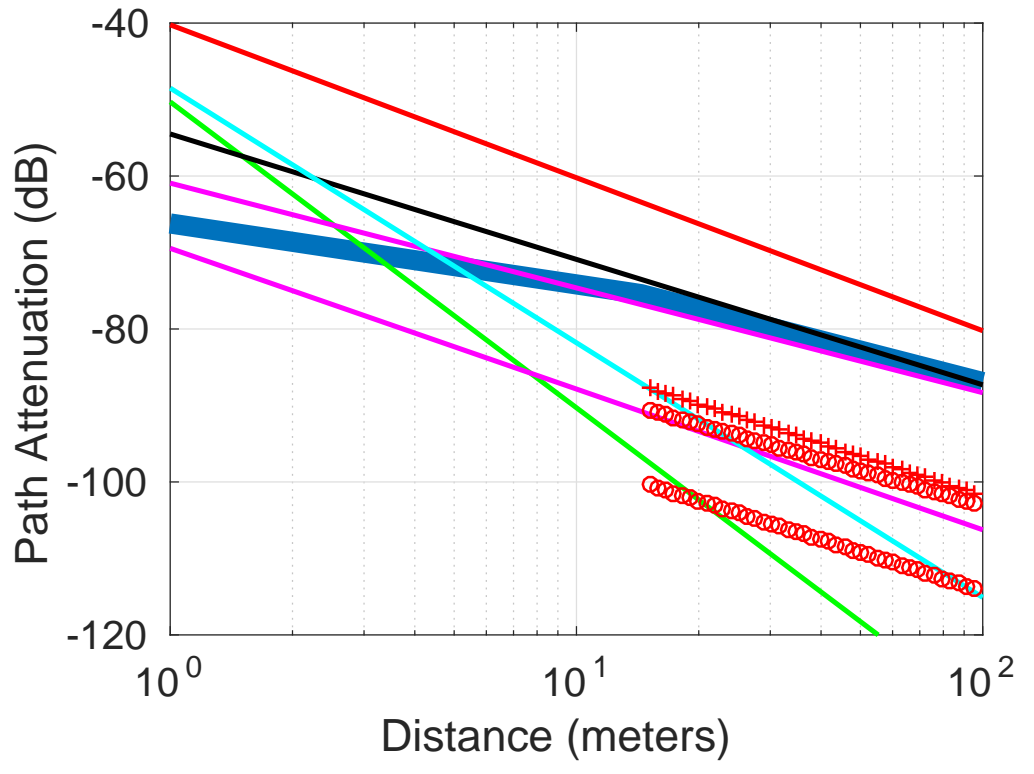

Figure 100: Comparison to other models: Long Polarization, 5.4GHz, T2, Low.

\begin{tabular}{c|c|c|c|c|c|c|c|c}
\hline Type & Frequency & Polarization & $\alpha_{1}$ & $\alpha_{2}$ & $\beta(\mathrm{m})$ & $k_{1}(\mathrm{~dB})$ & $k_{2}(\mathrm{~dB})$ & $\mathrm{MSE}$ \\
\hline \hline Overall & $5.4 \mathrm{GHz}$ & LongPol Low & 0.79698 & 1.3677 & 14.4068 & -66.2022 & -59.5904 & 11.1193 \\
\hline LOS & $5.4 \mathrm{GHz}$ & LongPol Low & 0.19605 & 7.6672 & 14.3712 & -69.4231 & 17.0553 & 8.5566 \\
\hline NLOS & $5.4 \mathrm{GHz}$ & LongPol Low & -1.041 & 1.2328 & 12.2267 & -86.38 & -61.657 & 10.6606 \\
\hline
\end{tabular}

Table 13: Parameters for Long Polarization, 5GHz, T2, Low 


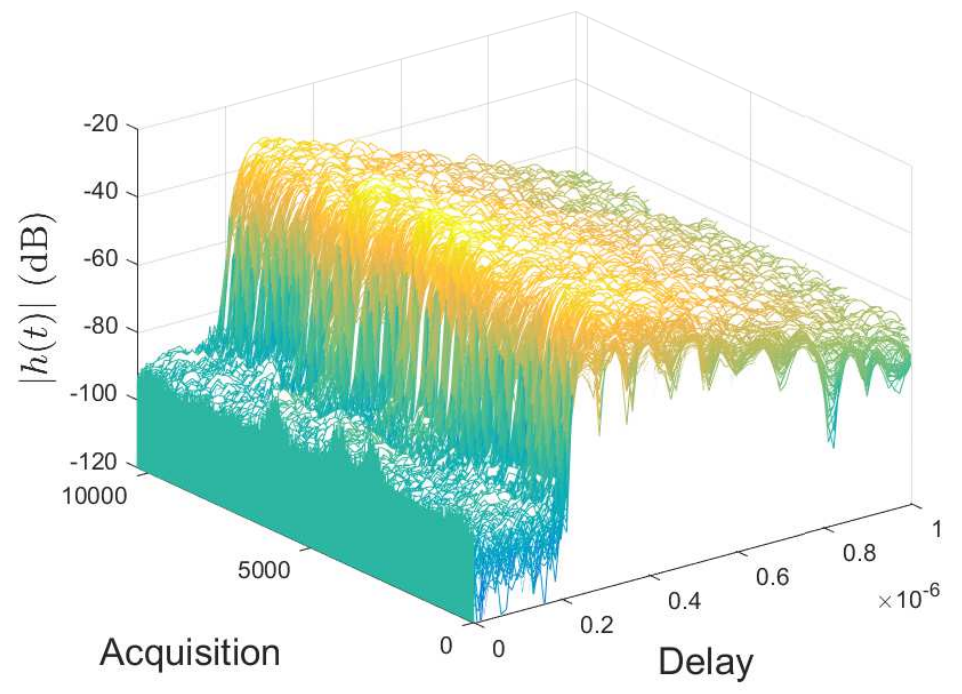

Figure 101: Impulse Responses: Long Polarization, 5.4GHz, T2, Low.

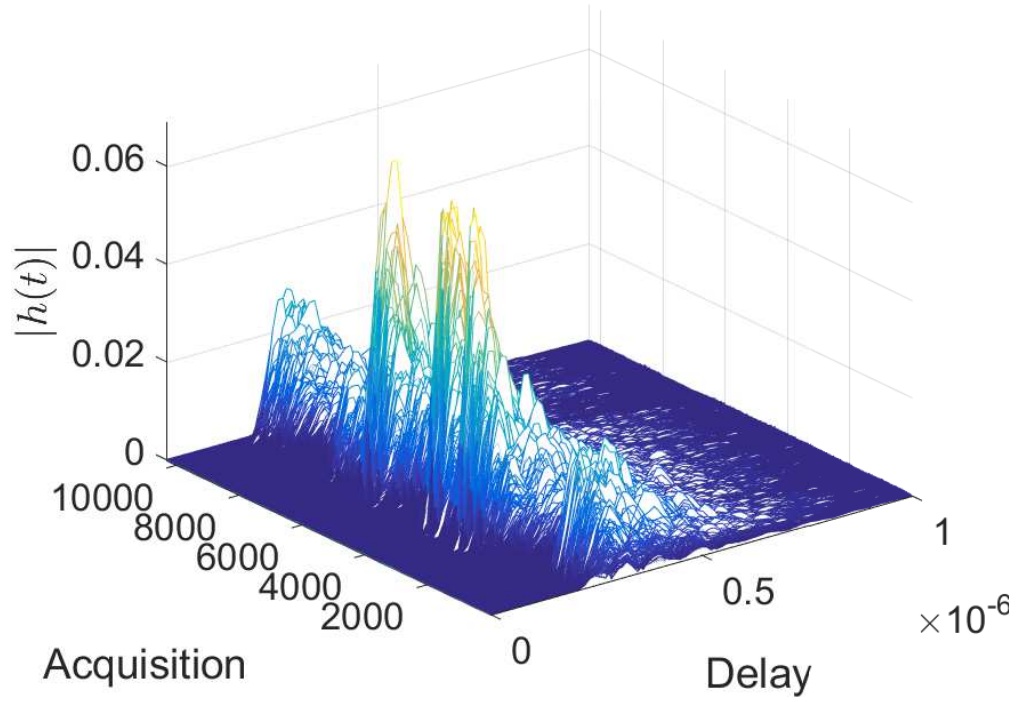

Figure 102: Impulse Responses: Long Polarization, 5.4GHz, T2, Low. 


\section{A.13 Vertical Polarization, 2.245GHz, Transmitter 1}

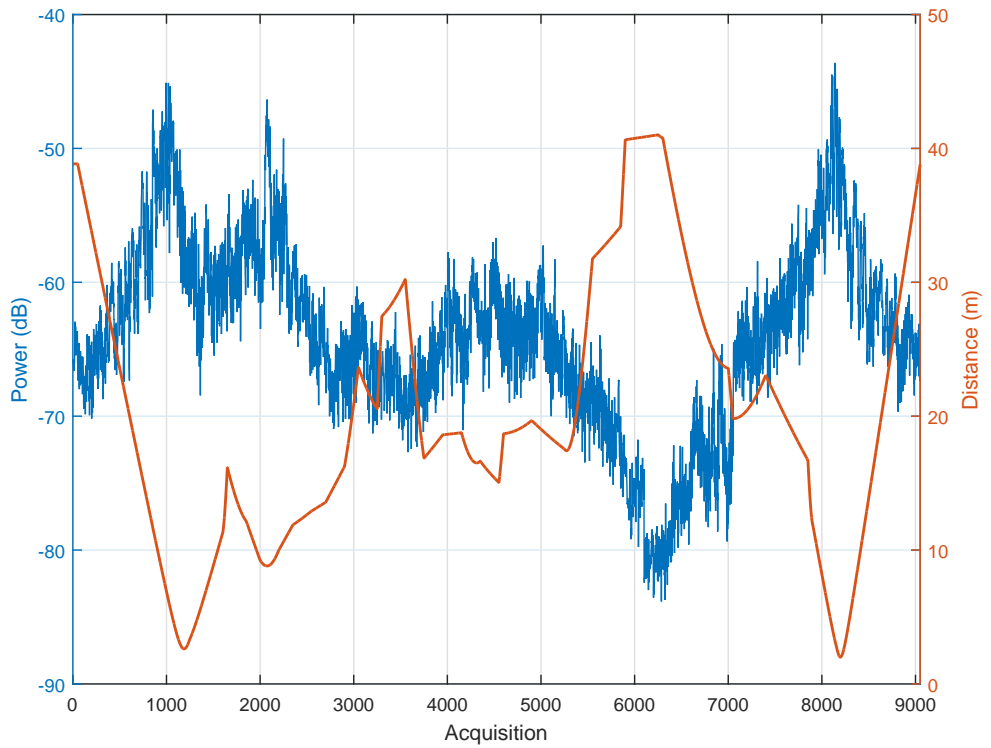

Figure 103: Overall Received Power vs. Acquisition Number and Distance vs. Acquisition Number: Vert Polarization, 2.245GHz, T1.

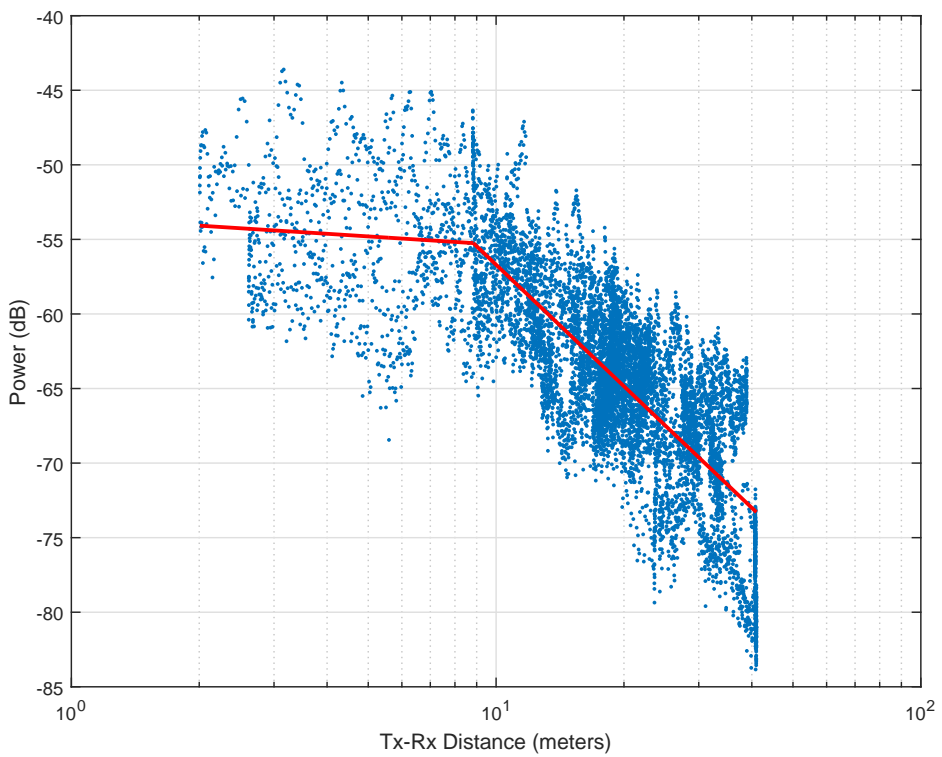

Figure 104: Overall Received Power vs. Distance: Vert Polarization, 2.245GHz, T1. 


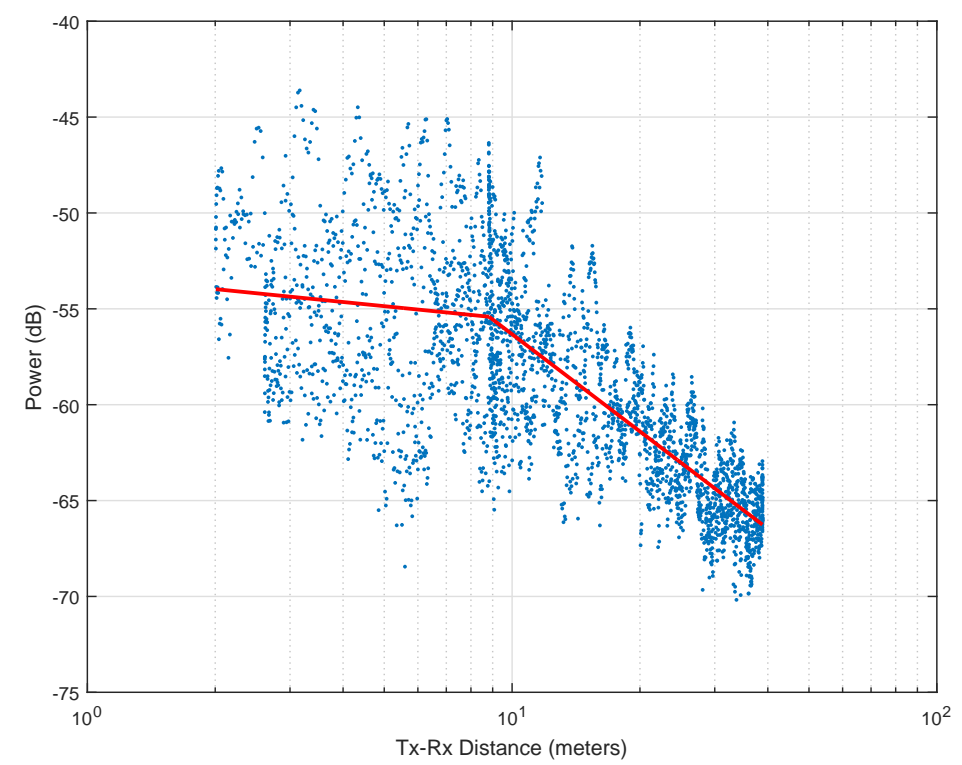

Figure 105: Line of Sight Received Power vs. Distance: Vert Polarization, 2.245GHz, $\mathrm{T} 1$.

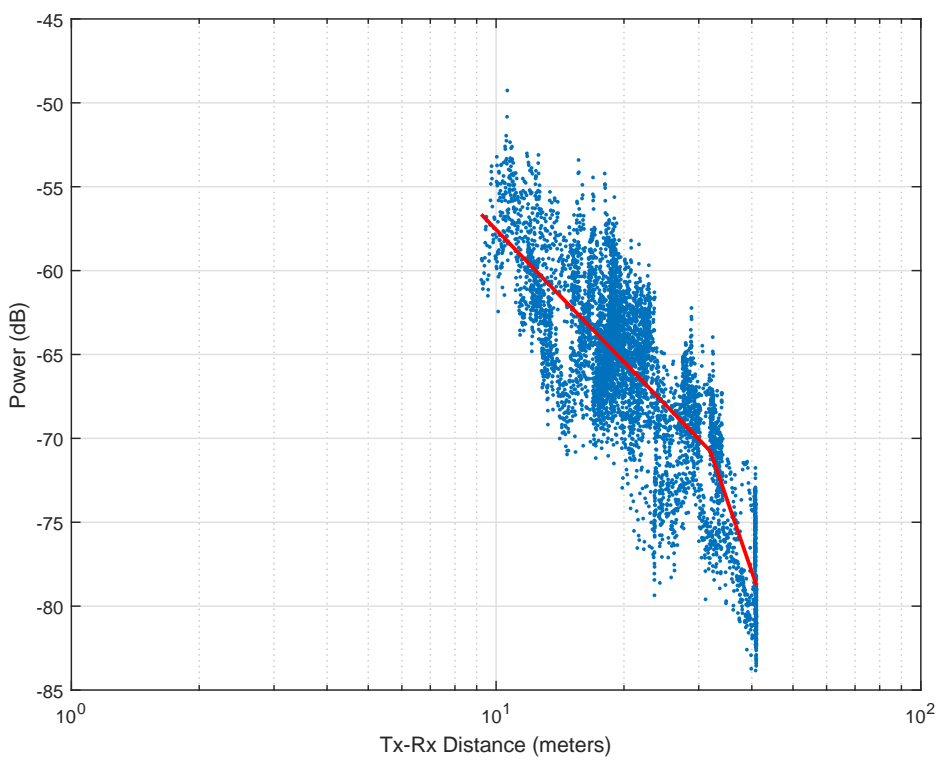

Figure 106: Non Line of Sight Received Power vs. Distance: Vert Polarization, 2.245GHz, T1. 


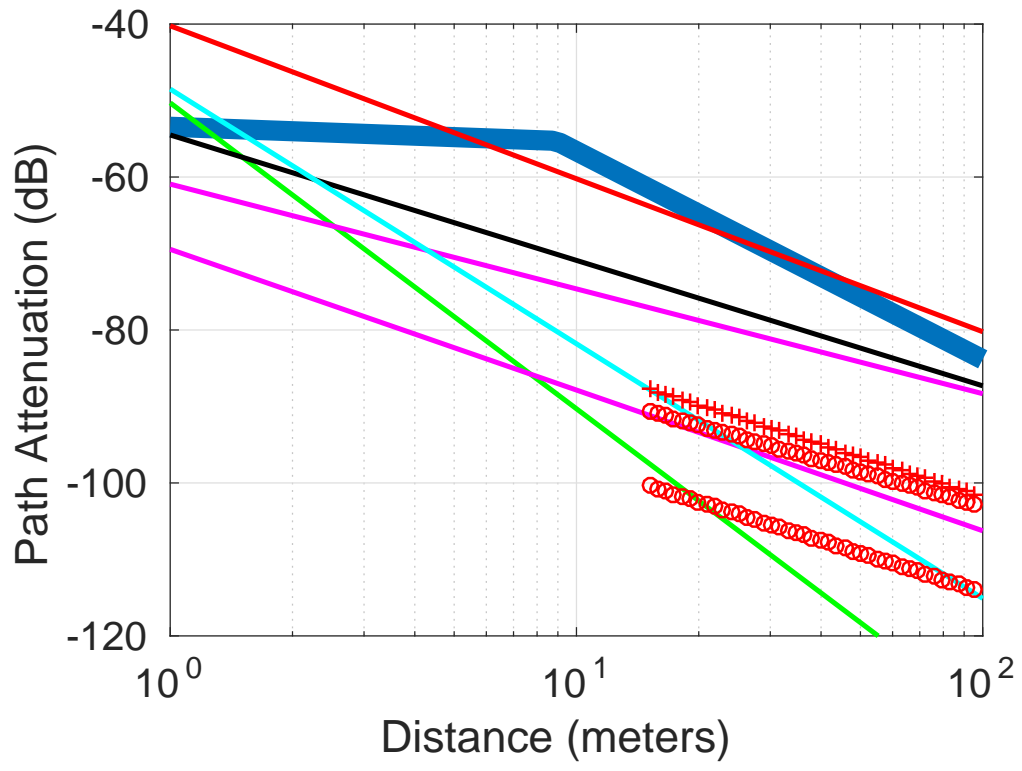

Figure 107: Comparison to other models: Vert Polarization, 2.245GHz, T1.

\begin{tabular}{c|c|c|c|c|c|c|c|c}
\hline Type & Frequency & Polarization & $\alpha_{1}$ & $\alpha_{2}$ & $\beta(\mathrm{m})$ & $k_{1}(\mathrm{~dB})$ & $k_{2}(\mathrm{~dB})$ & MSE \\
\hline \hline Overall & $2.245 \mathrm{GHz}$ & Vpol & 0.19369 & 2.7212 & 8.9123 & -53.4288 & -29.4182 & 12.1805 \\
\hline LOS & $2.245 \mathrm{GHz}$ & Vpol & 0.22358 & 1.6849 & 8.8277 & -53.2983 & -39.4768 & 11.0969 \\
\hline NLOS & $2.245 \mathrm{GHz}$ & Vpol & 2.6179 & 7.3565 & 31.9439 & -31.3789 & 39.9079 & 10.1757 \\
\hline
\end{tabular}

Table 14: Parameters for Vert Polarization, 2.245GHz, T1 


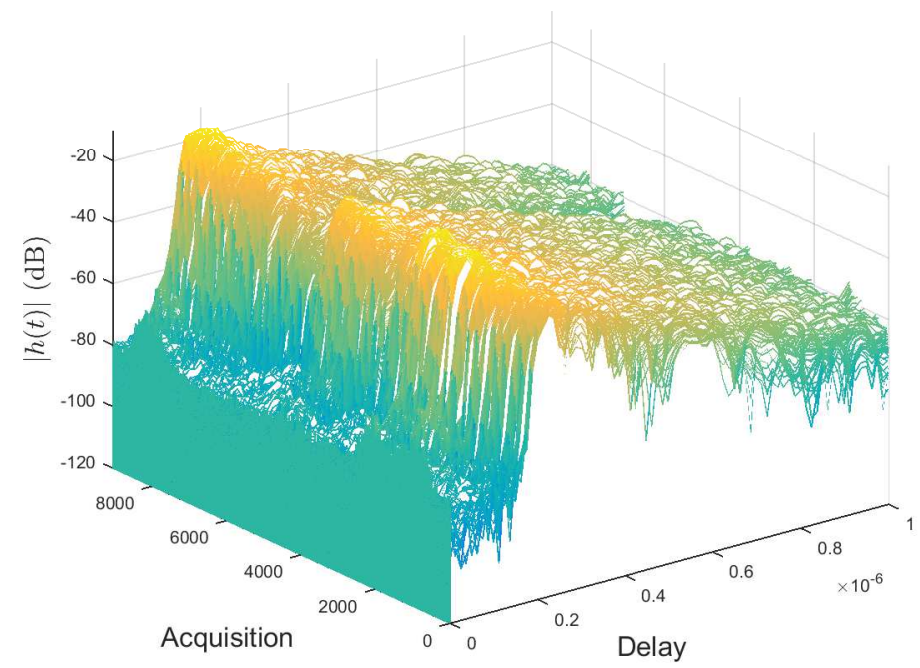

Figure 108: Impulse Responses: Vert Polarization, 2.245GHz, T1.

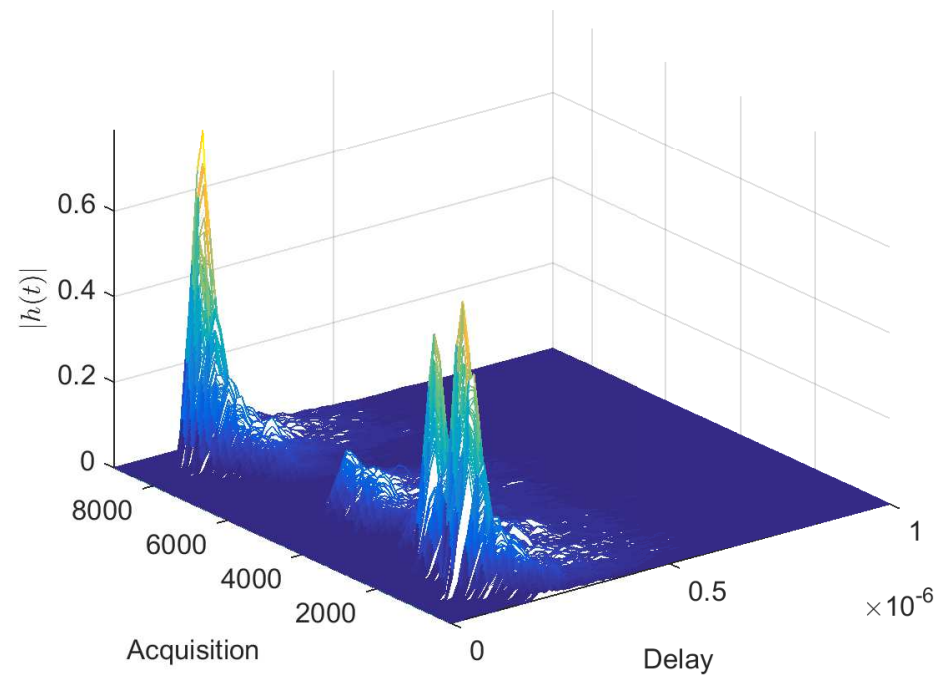

Figure 109: Impulse Responses: Vert Polarization, 2.245GHz, T1. 


\section{A.14 Vertical Polarization, 5GHz, Transmitter 1}

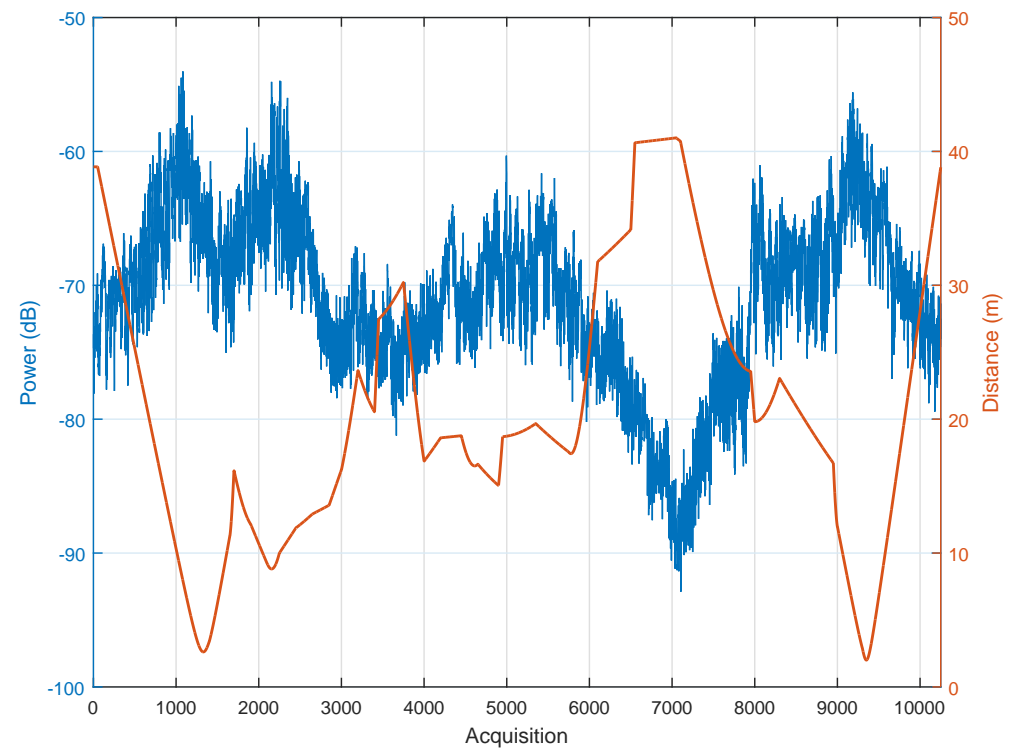

Figure 110: Overall Received Power vs. Acquisition Number and Distance vs. Acquisition Number: Vert Polarization, 5.4GHz, T1.

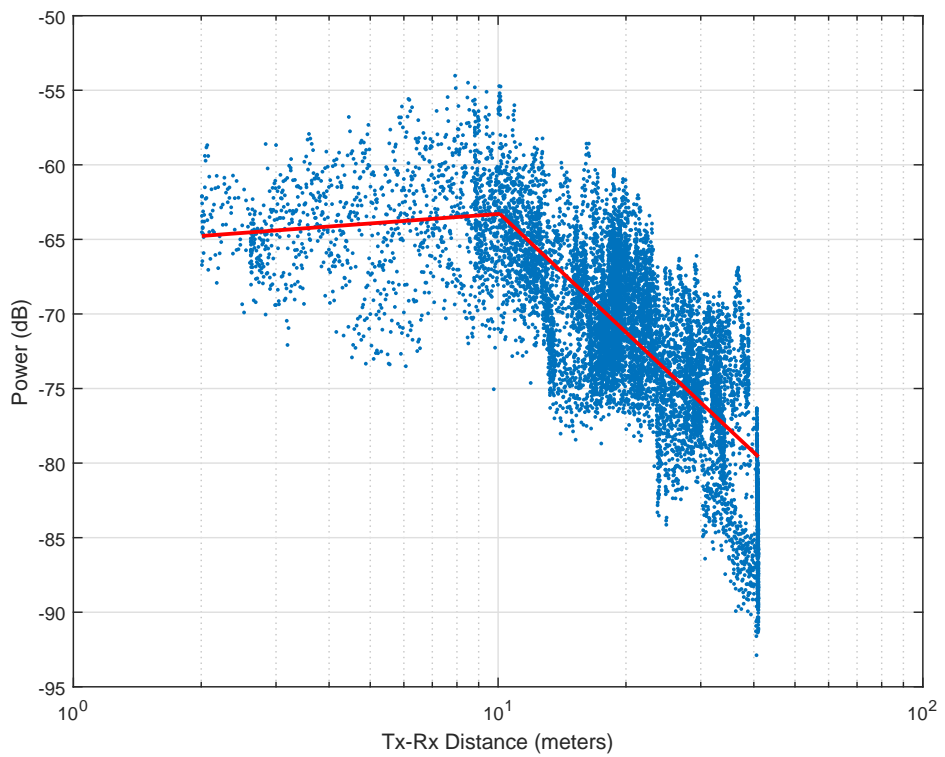

Figure 111: Overall Received Power vs. Distance: Vert Polarization, 5.4GHz, T1. 


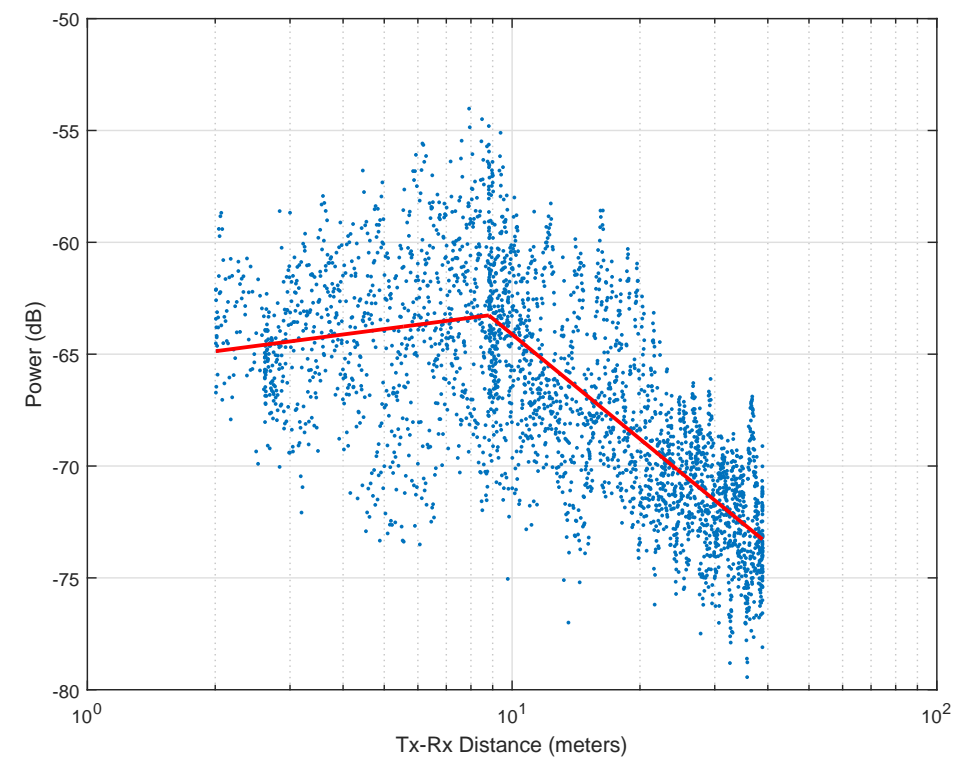

Figure 112: Line of Sight Received Power vs. Distance: Vert Polarization, 5.4GHz, T1.

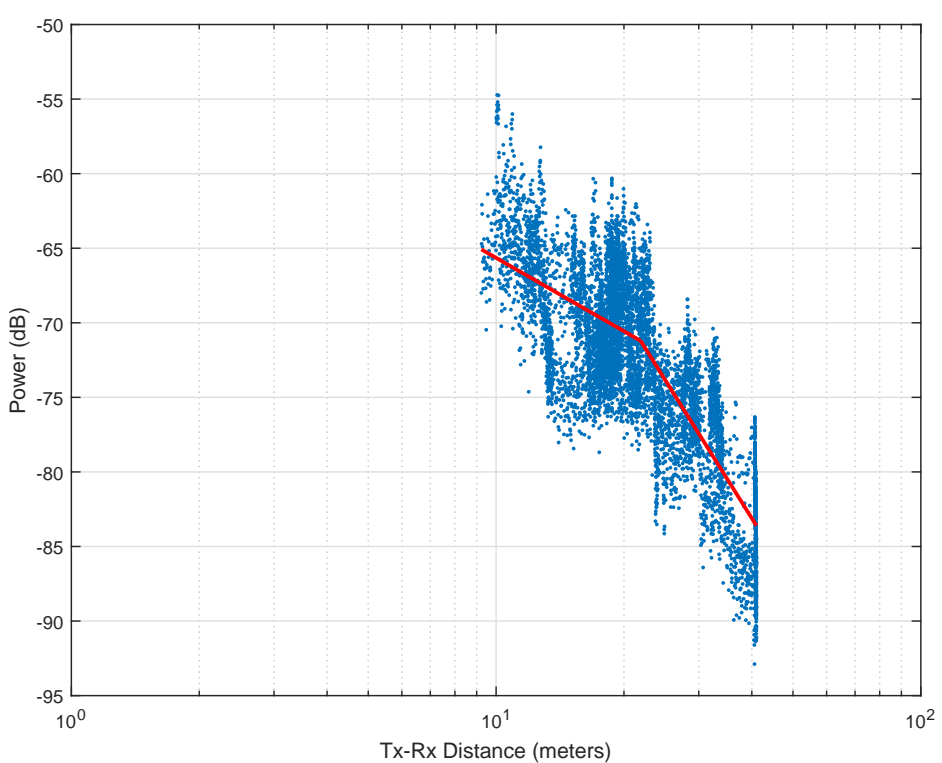

Figure 113: Non Line of Sight Received Power vs. Distance: Vert Polarization, 5.4GHz, $\mathrm{T} 1$. 


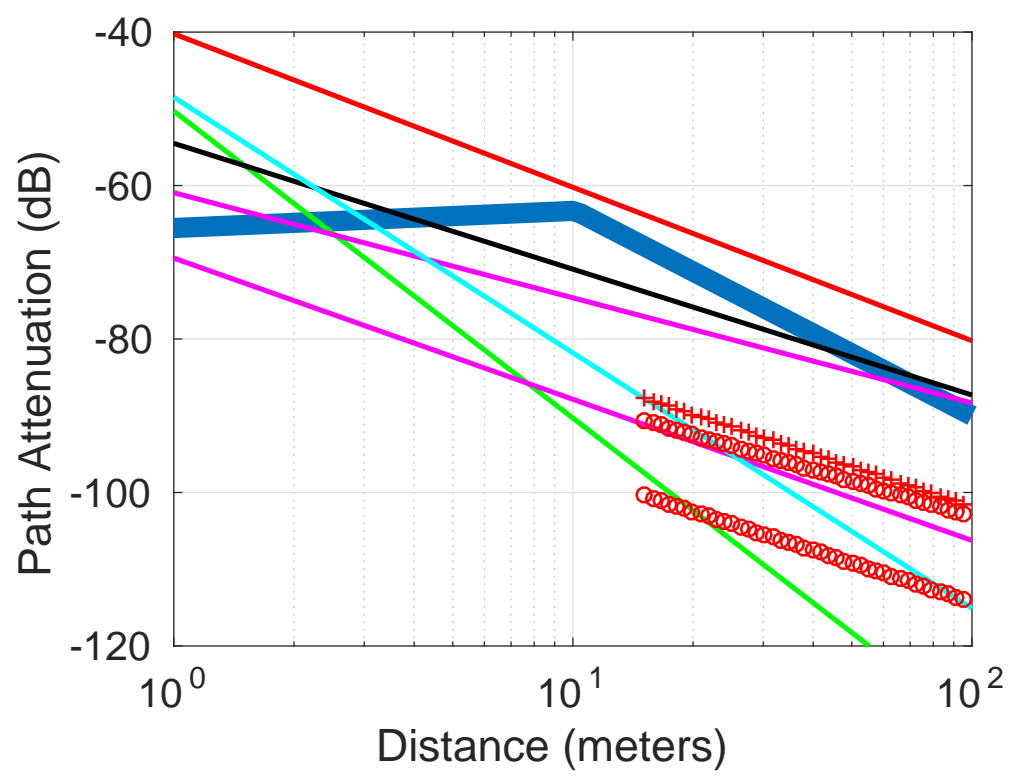

Figure 114: Comparison to other models: Vert Polarization, 5.4GHz, T1.

\begin{tabular}{c|c|c|c|c|c|c|c|c}
\hline Type & Frequency & Polarization & $\alpha_{1}$ & $\alpha_{2}$ & $\beta(\mathrm{m})$ & $k_{1}(\mathrm{~dB})$ & $k_{2}(\mathrm{~dB})$ & MSE \\
\hline \hline Overall & $5.4 \mathrm{GHz}$ & Vpol & -0.22296 & 2.6873 & 10.1526 & -65.5349 & -36.2409 & 12.0084 \\
\hline LOS & $5.4 \mathrm{GHz}$ & Vpol & -0.24922 & 1.5547 & 8.8181 & -65.6198 & -48.566 & 10.1366 \\
\hline NLOS & $5.4 \mathrm{GHz}$ & Vpol & 1.6346 & 4.5464 & 21.8949 & -49.2994 & -10.2715 & 10.9476 \\
\hline
\end{tabular}

Table 15: Parameters for Vert Polarization, 5GHz, T1 


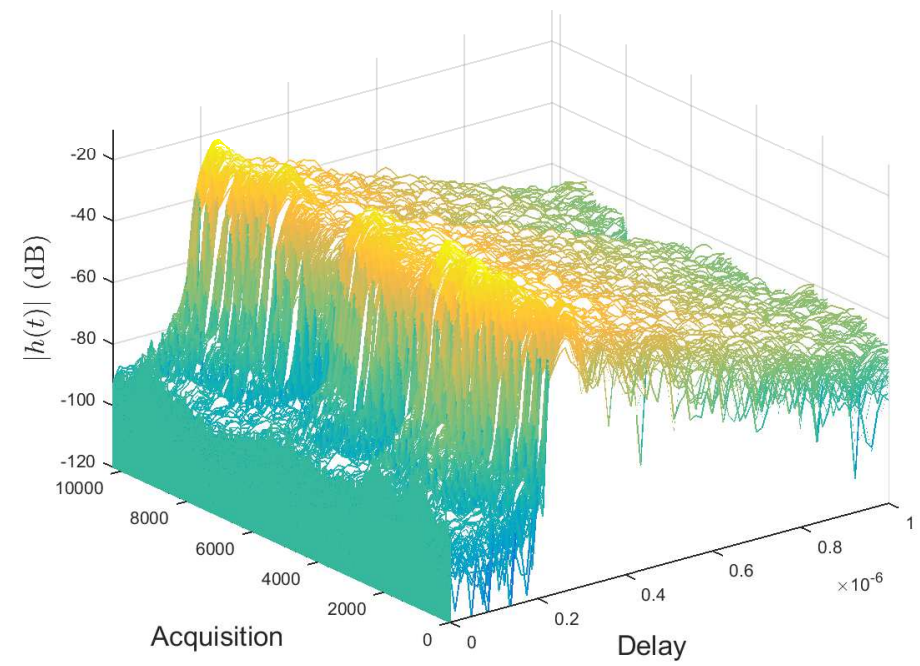

Figure 115: Impulse Responses: Vert Polarization, 5.4GHz, T1.

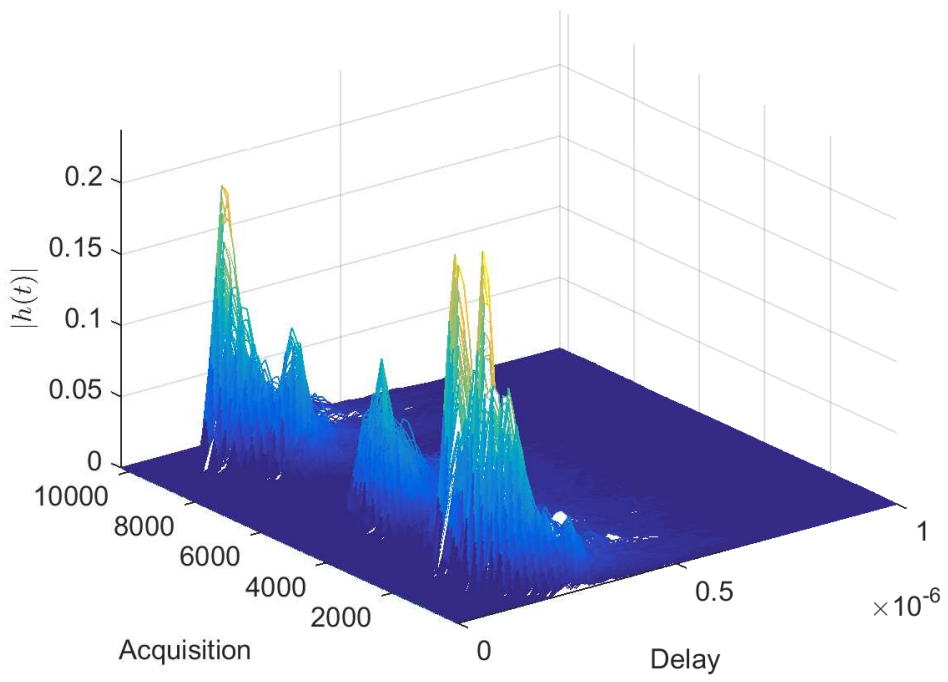

Figure 116: Impulse Responses: Vert Polarization, 5.4GHz, T1. 


\section{A.15 Vertical Polarization, 2.245GHz, Transmitter 2, High}

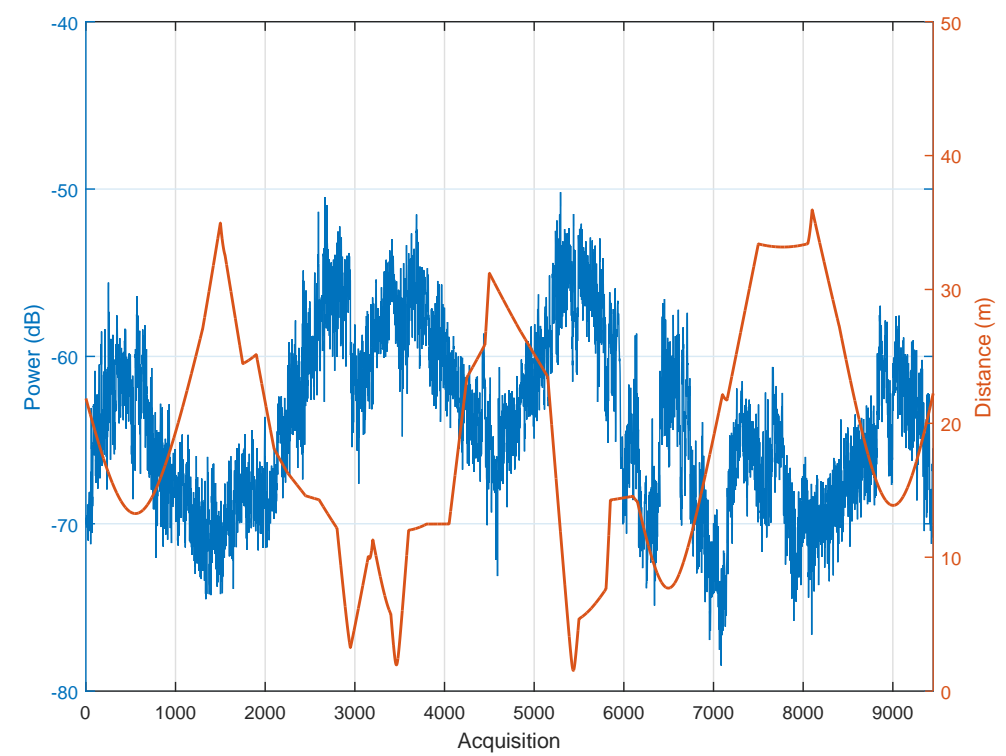

Figure 117: Overall Received Power vs. Acquisition Number and Distance vs. Acquisition Number: Vert Polarization, 2.245GHz, T2, High.

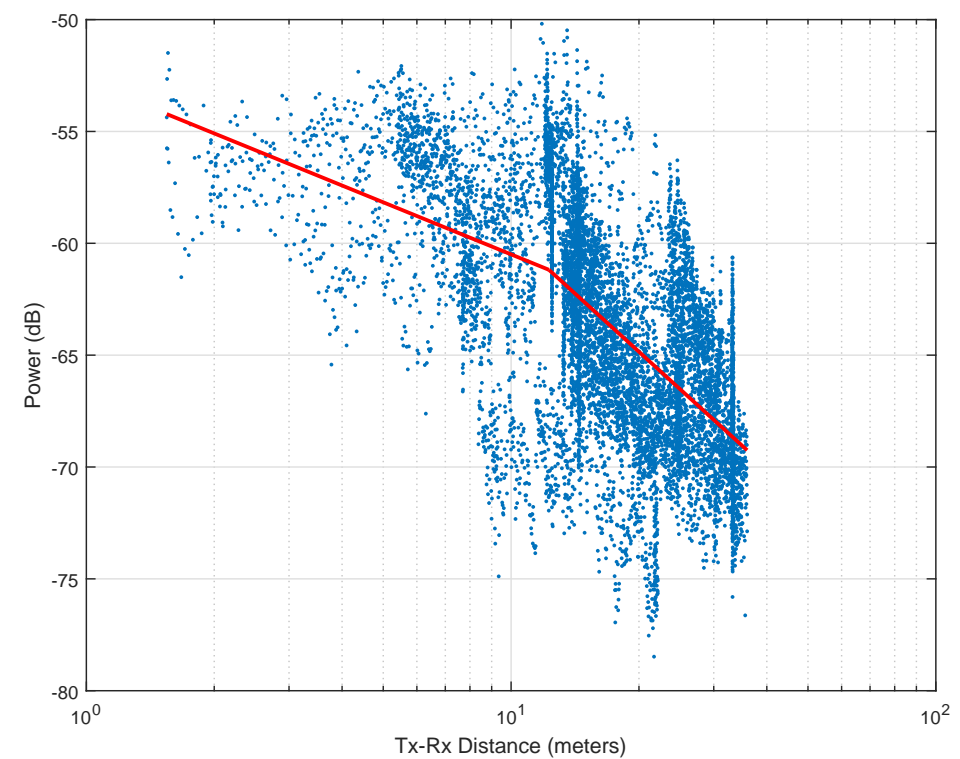

Figure 118: Overall Received Power vs. Distance: Vert Polarization, 2.245GHz, T2, High. 


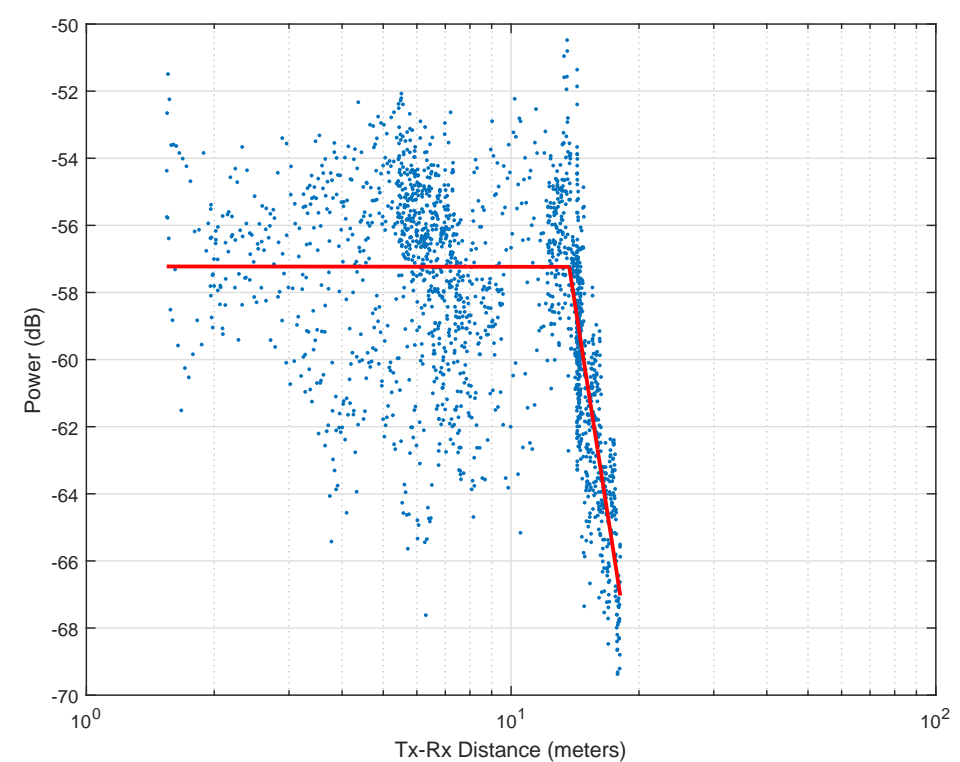

Figure 119: Line of Sight Received Power vs. Distance: Vert Polarization, 2.245GHz, T2, High.

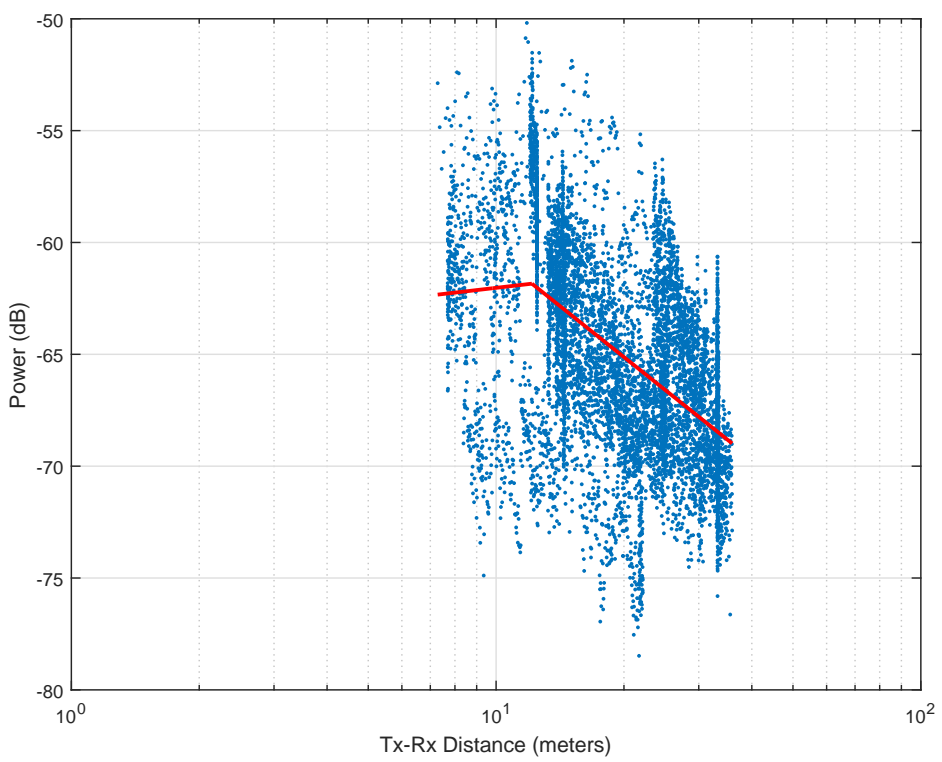

Figure 120: Non Line of Sight Received Power vs. Distance: Vert Polarization, 2.245GHz, T2, High. 


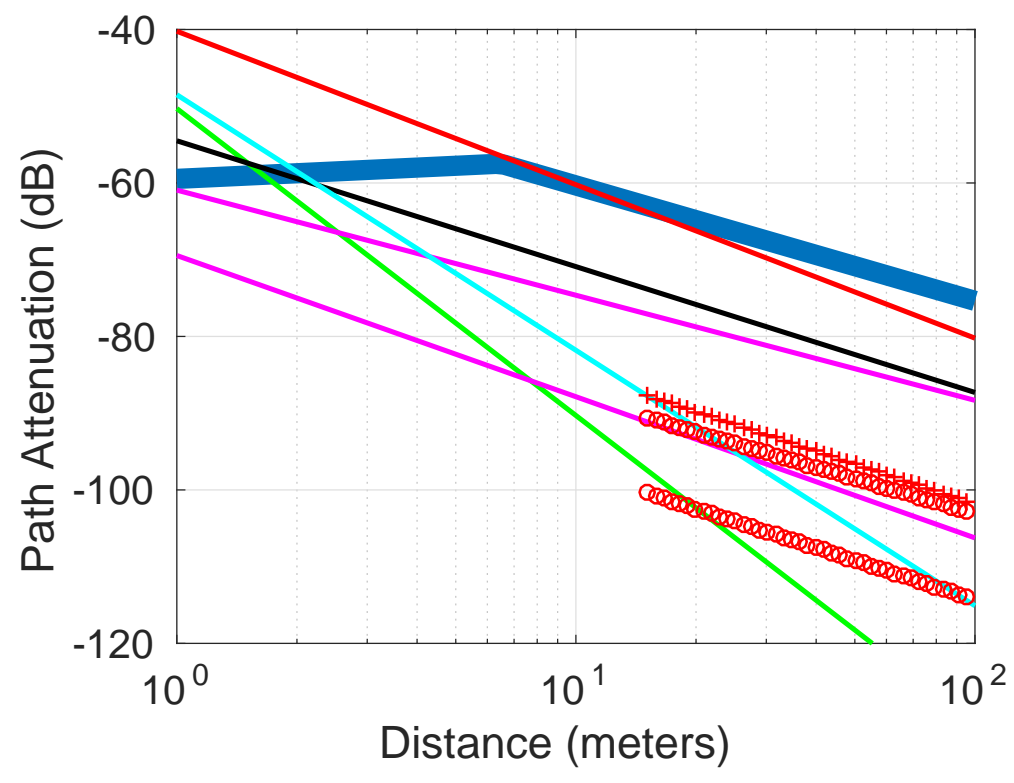

Figure 121: Comparison to other models: Vert Polarization, 2.245GHz, T2, High.

\begin{tabular}{c|c|c|c|c|c|c|c|c}
\hline Type & Frequency & Polarization & $\alpha_{1}$ & $\alpha_{2}$ & $\beta(\mathrm{m})$ & $k_{1}(\mathrm{~dB})$ & $k_{2}(\mathrm{~dB})$ & $\mathrm{MSE}$ \\
\hline \hline Overall & $2.245 \mathrm{GHz}$ & Vpol High & -0.24426 & 1.5046 & 6.4786 & -59.4669 & -45.2756 & 12.1637 \\
\hline LOS & $2.245 \mathrm{GHz}$ & Vpol High & 0.0012037 & 8.1405 & 13.6994 & -57.222 & 35.298 & 8.0396 \\
\hline NLOS & $2.245 \mathrm{GHz}$ & Vpol High & -0.22064 & 1.5146 & 12.1426 & -64.2343 & -45.4186 & 11.9743 \\
\hline
\end{tabular}

Table 16: Parameters for Vert Polarization, 2.245GHz, T2, High 


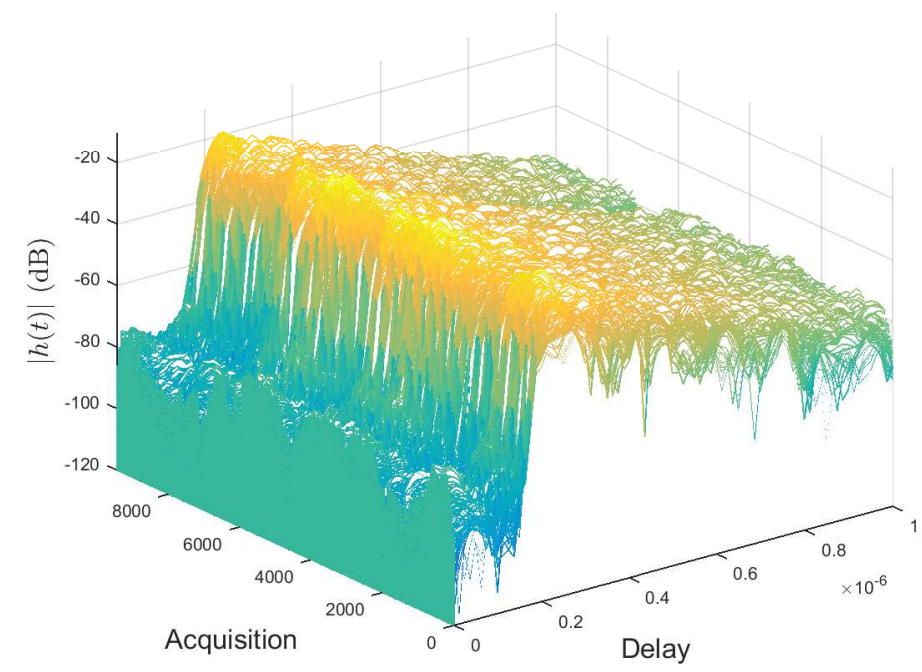

Figure 122: Impulse Responses: Vert Polarization, 2.245GHz, T2, High.

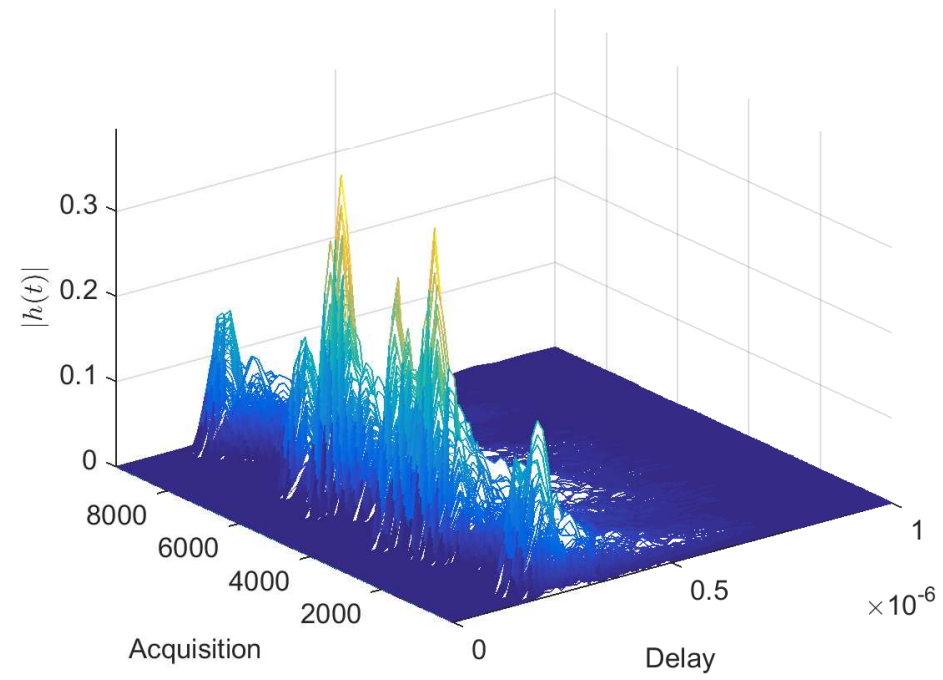

Figure 123: Impulse Responses: Vert Polarization, 2.245GHz, T2, High. 


\section{A.16 Vertical Polarization, 2.245GHz, Transmitter 2, Low}

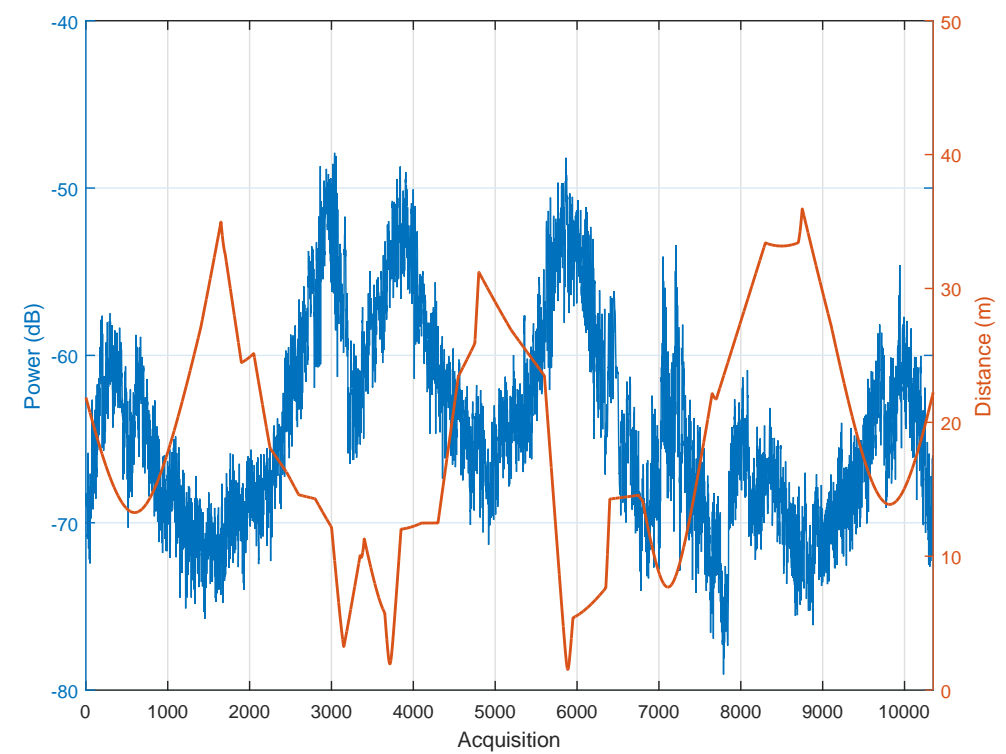

Figure 124: Overall Received Power vs. Acquisition Number and Distance vs. Acquisition Number: Vert Polarization, 2.245GHz, T2, Low.

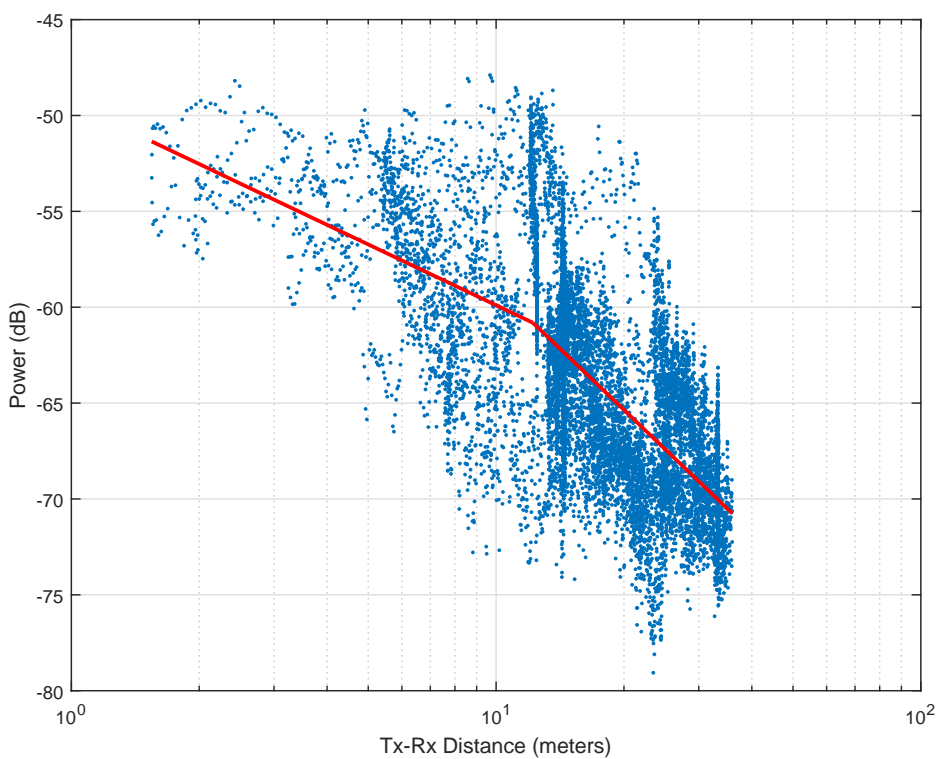

Figure 125: Overall Received Power vs. Acquisition Number and Distance vs. Acquisition Number: Vert Polarization, 2.245GHz, T2, Low. 


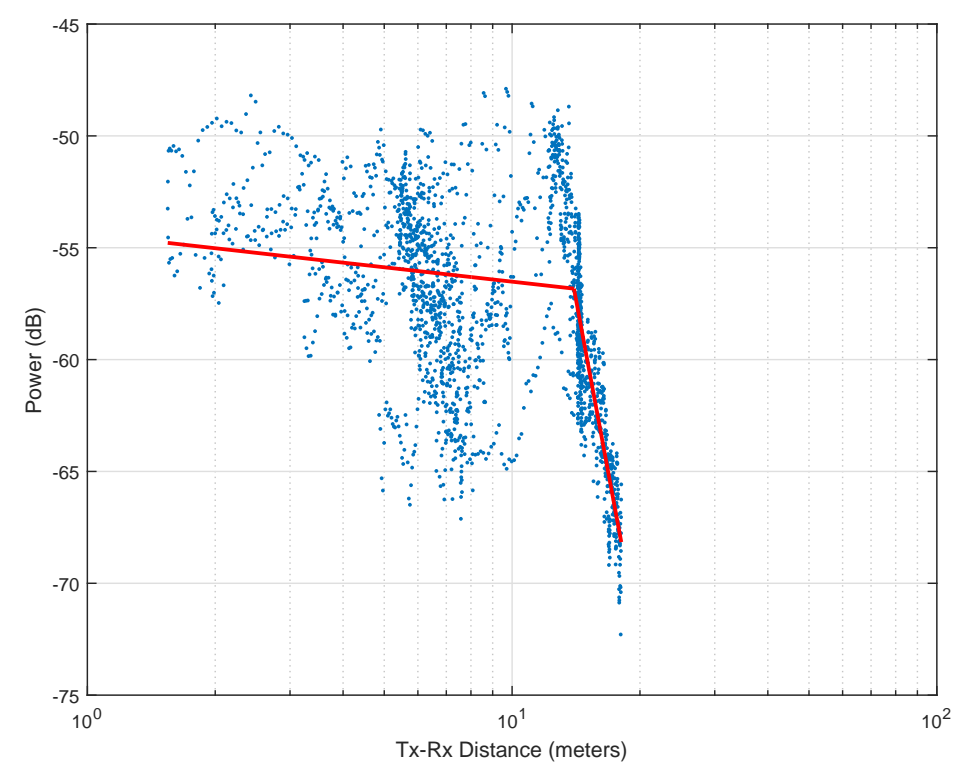

Figure 126: Line of Sight Received Power vs. Acquisition Number and Distance vs. Acquisition Number: Vert Polarization, 2.245GHz, T2, Low.

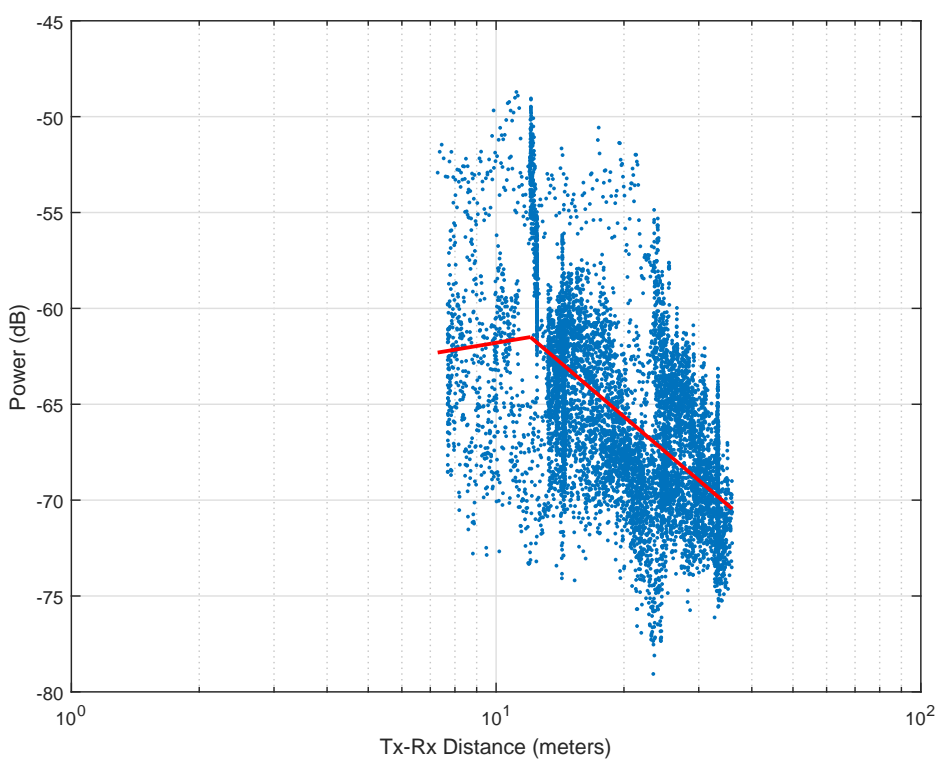

Figure 127: Non Line of Sight Received Power vs. Acquisition Number and Distance vs. Acquisition Number: Vert Polarization, 2.245GHz, T2, Low. 


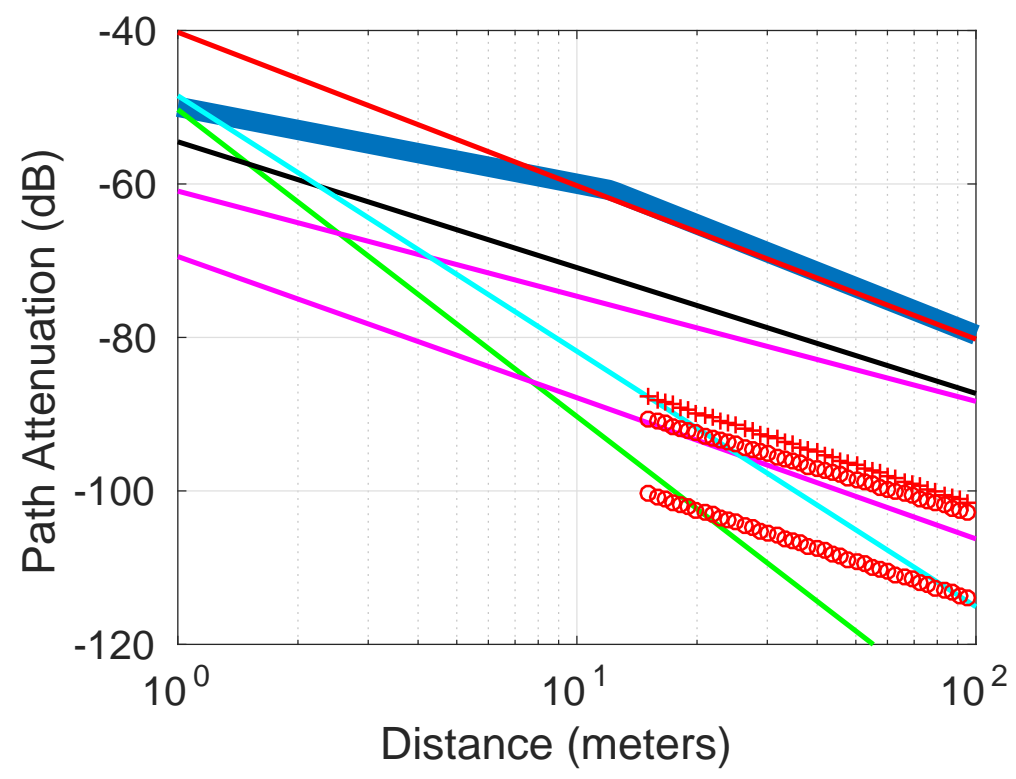

Figure 128: Comparison to other models: Vert Polarization, 2.245GHz, T2, Low.

\begin{tabular}{c|c|c|c|c|c|c|c|c}
\hline Type & Frequency & Polarization & $\alpha_{1}$ & $\alpha_{2}$ & $\beta(\mathrm{m})$ & $k_{1}(\mathrm{~dB})$ & $k_{2}(\mathrm{~dB})$ & MSE \\
\hline \hline Overall & $2.245 \mathrm{GHz}$ & Vpol Low & 1.0029 & 2.0598 & 12.1722 & -49.9412 & -38.4701 & 12.8307 \\
\hline LOS & $2.245 \mathrm{GHz}$ & Vpol Low & 0.21386 & 10.171 & 13.9837 & -54.3781 & 59.6931 & 10.7806 \\
\hline NLOS & $2.245 \mathrm{GHz}$ & Vpol Low & -0.36991 & 1.8857 & 12.0498 & -65.4945 & -41.1119 & 12.2427 \\
\hline
\end{tabular}

Table 17: Parameters for Vert Polarization, 2.245GHz, T2, Low 


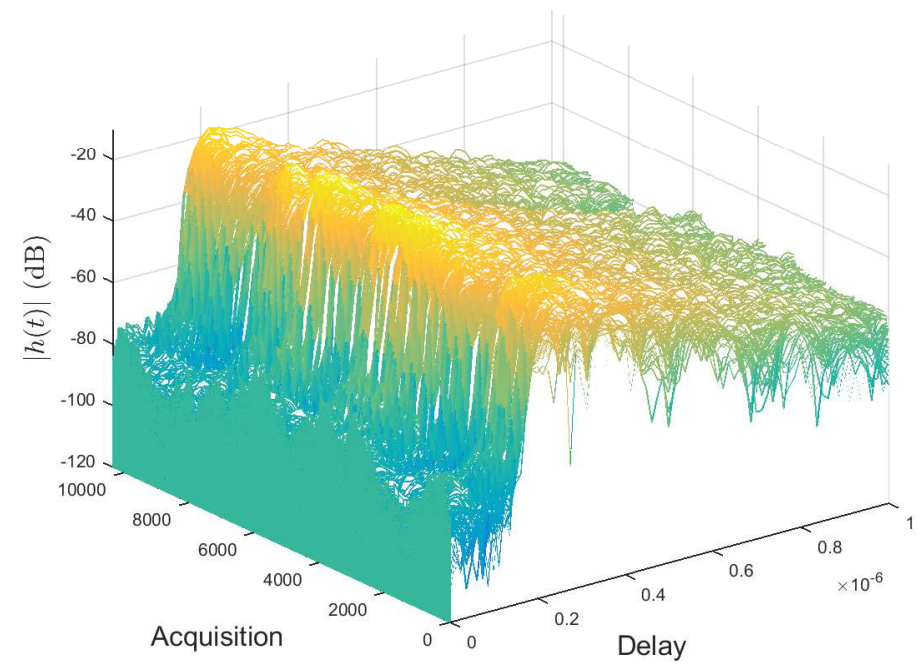

Figure 129: Impulse Responses: Vert Polarization, 2.245GHz, T2, Low.

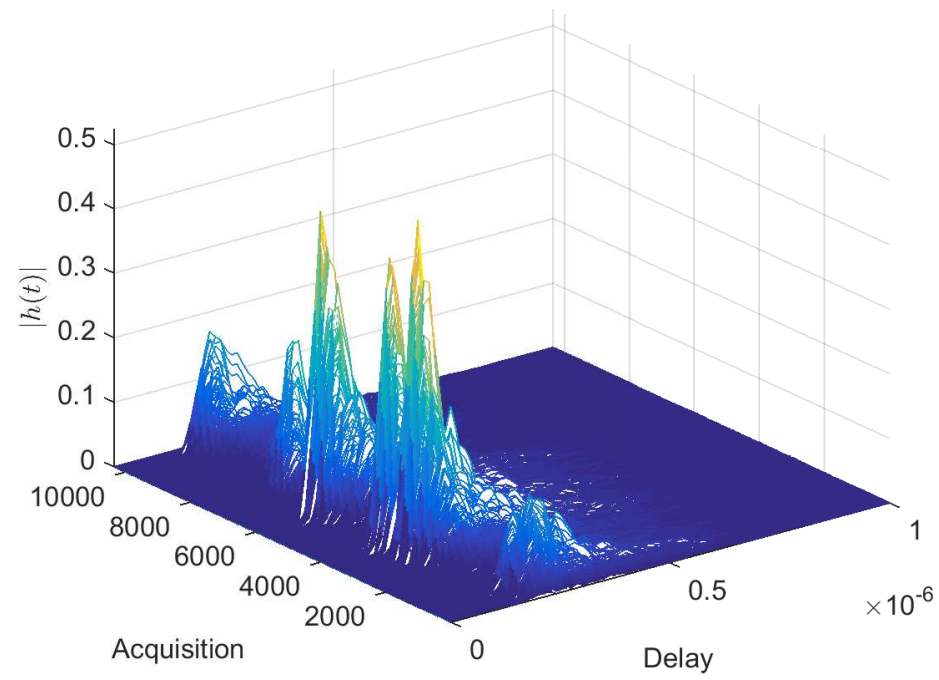

Figure 130: Impulse Responses: Vert Polarization, 2.245GHz, T2, Low. 


\section{A.17 Vertical Polarization, 5GHz, Transmitter 2, High}

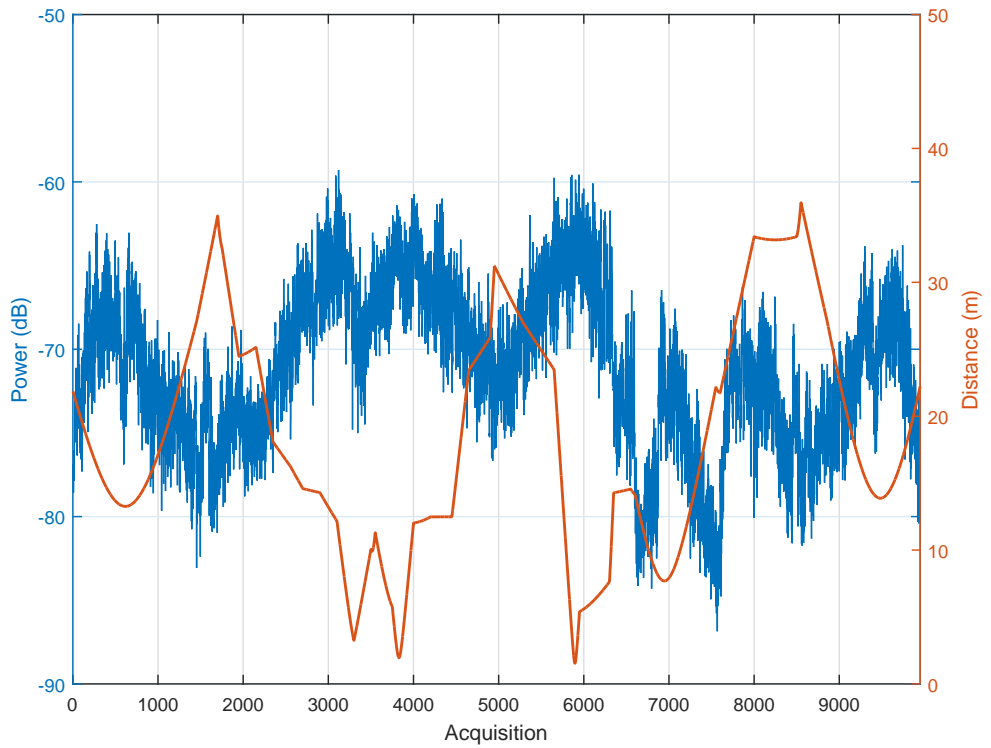

Figure 131: Overall Received Power vs. Acquisition Number and Distance vs. Acquisition Number: Vert Polarization, 5.4GHz, T2, High.

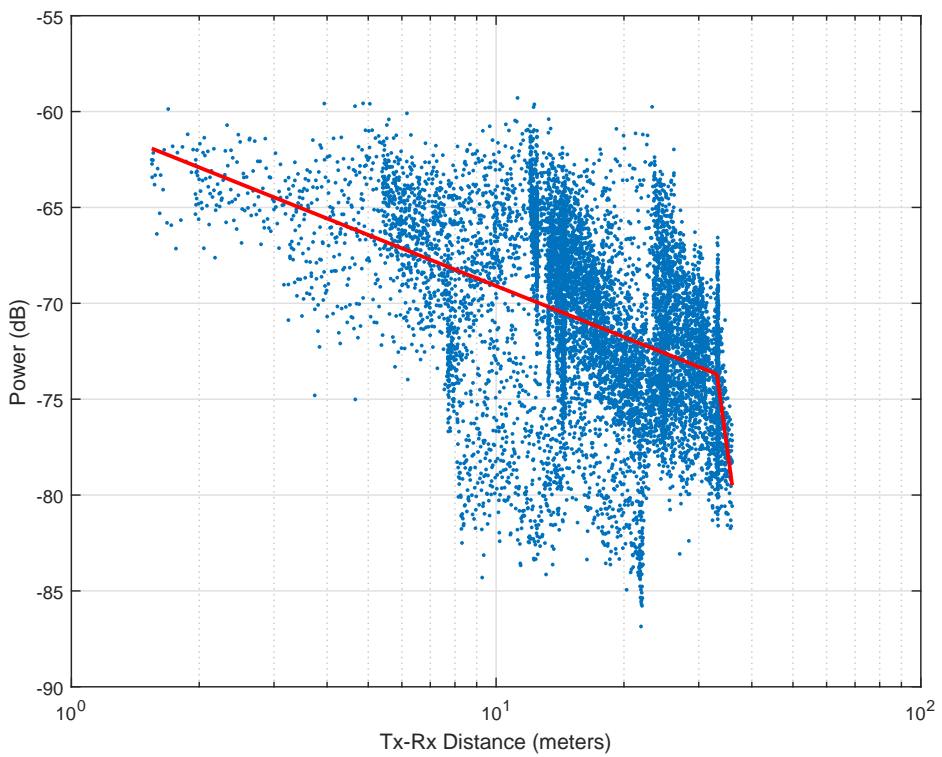

Figure 132: Overall Received Power vs. Distance: Vert Polarization, 5.4GHz, T2, High. 


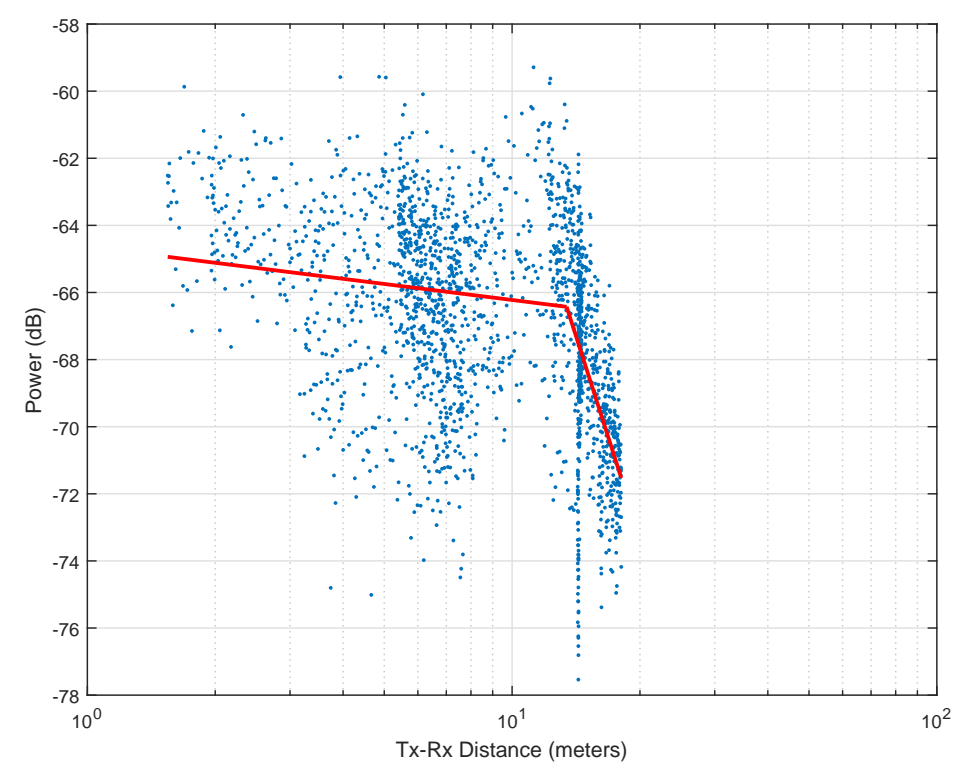

Figure 133: Line of Sight Received Power vs. Distance: Vert Polarization, 5.4GHz, T2, High.

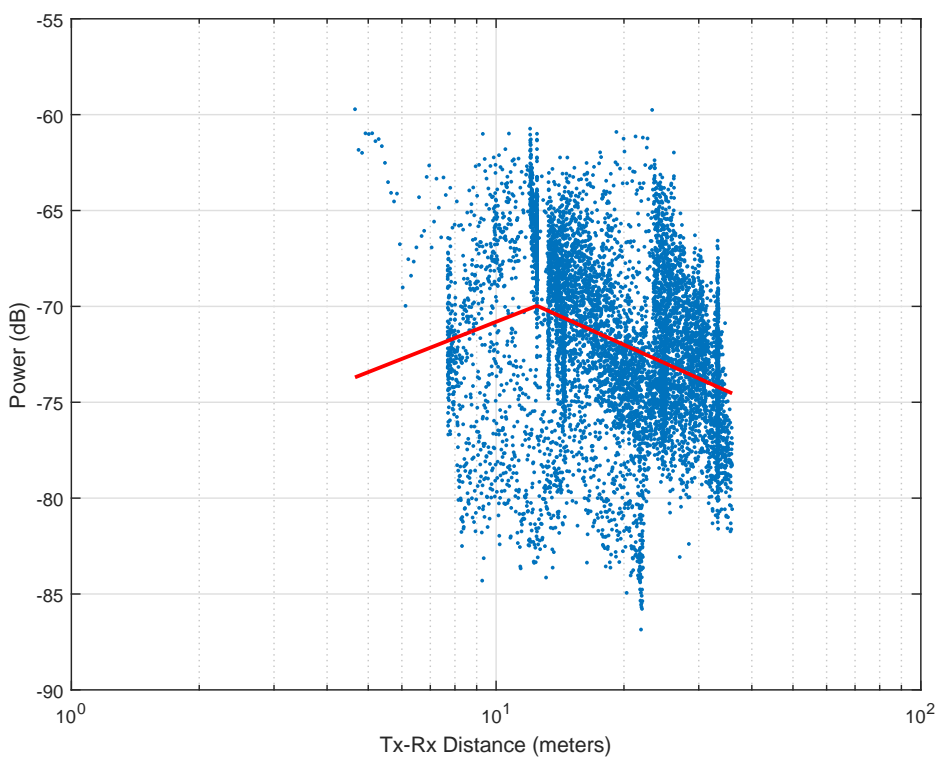

Figure 134: Non Line of Sight Received Power vs. Distance: Vert Polarization, 5.4GHz, T2, High. 


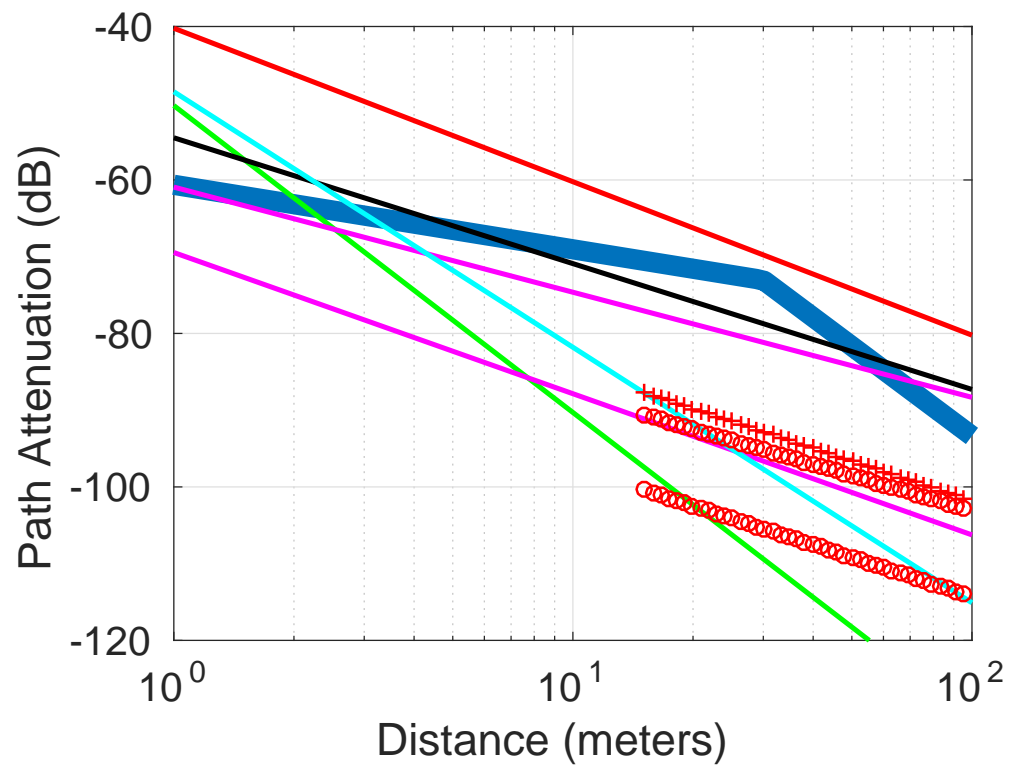

Figure 135: Comparison to other models: Vert Polarization, 5.4GHz, T2, High.

\begin{tabular}{c|c|c|c|c|c|c|c|c}
\hline Type & Frequency & Polarization & $\alpha_{1}$ & $\alpha_{2}$ & $\beta(\mathrm{m})$ & $k_{1}(\mathrm{~dB})$ & $k_{2}(\mathrm{~dB})$ & MSE \\
\hline \hline Overall & $5.4 \mathrm{GHz}$ & Vpol High & 0.84074 & 3.8845 & 30.0082 & -60.6292 & -15.665 & 12.4382 \\
\hline LOS & $5.4 \mathrm{GHz}$ & Vpol High & 0.1585 & 3.9643 & 13.4396 & -64.6383 & -21.6947 & 8.3161 \\
\hline NLOS & $5.4 \mathrm{GHz}$ & Vpol High & -0.87168 & 0.99421 & 12.4772 & -79.5216 & -59.0693 & 12.5158 \\
\hline
\end{tabular}

Table 18: Parameters for Vert Polarization, 5GHz, T2, High 


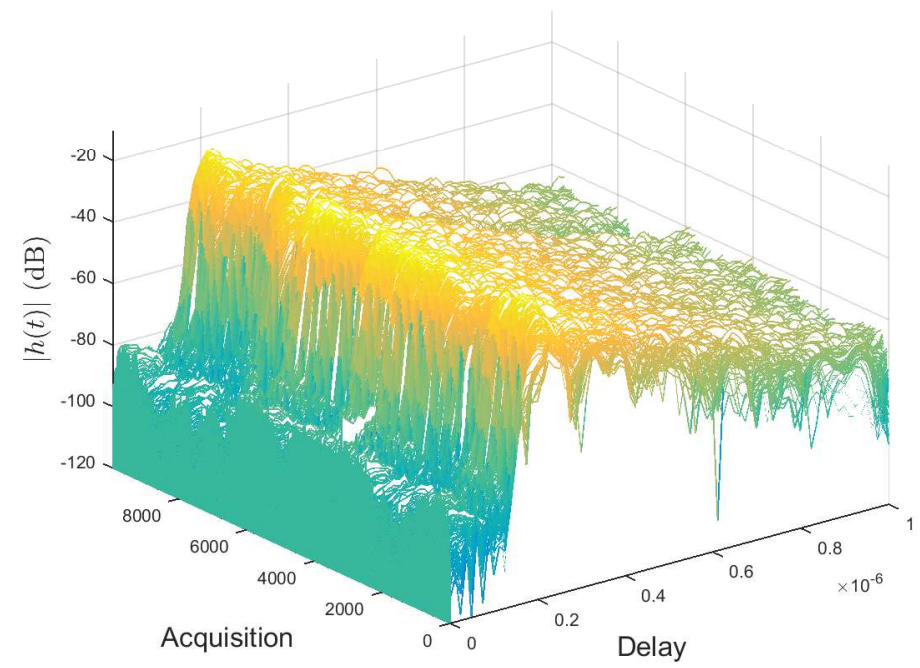

Figure 136: Impulse Responses: Vert Polarization, 5.4GHz, T2, High.

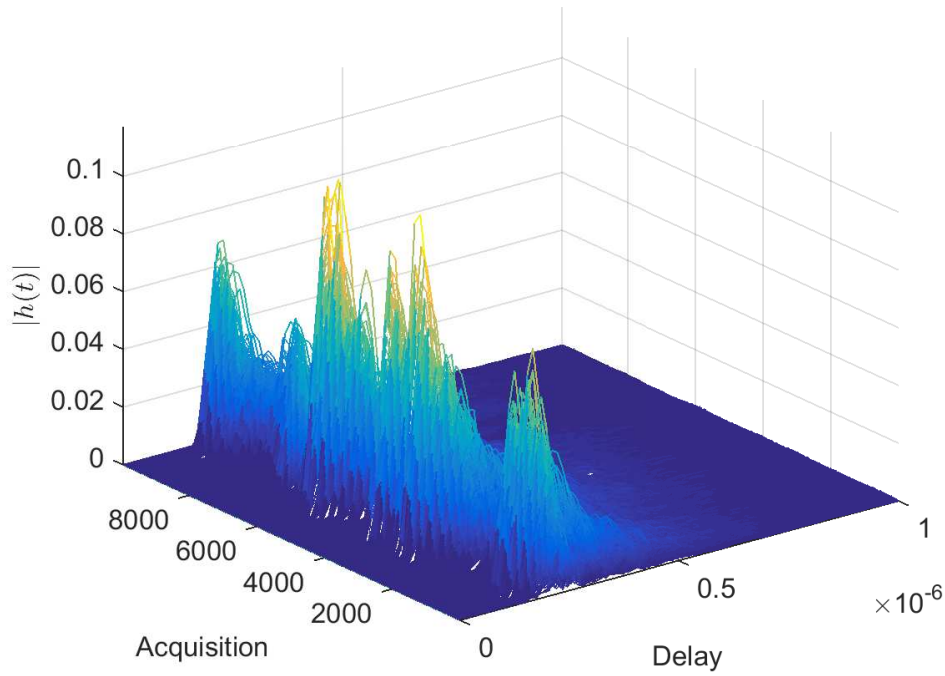

Figure 137: Impulse Responses: Vert Polarization, 5.4GHz, T2, High. 


\section{A.18 Vertical Polarization, 5GHz, Transmitter 2, Low}

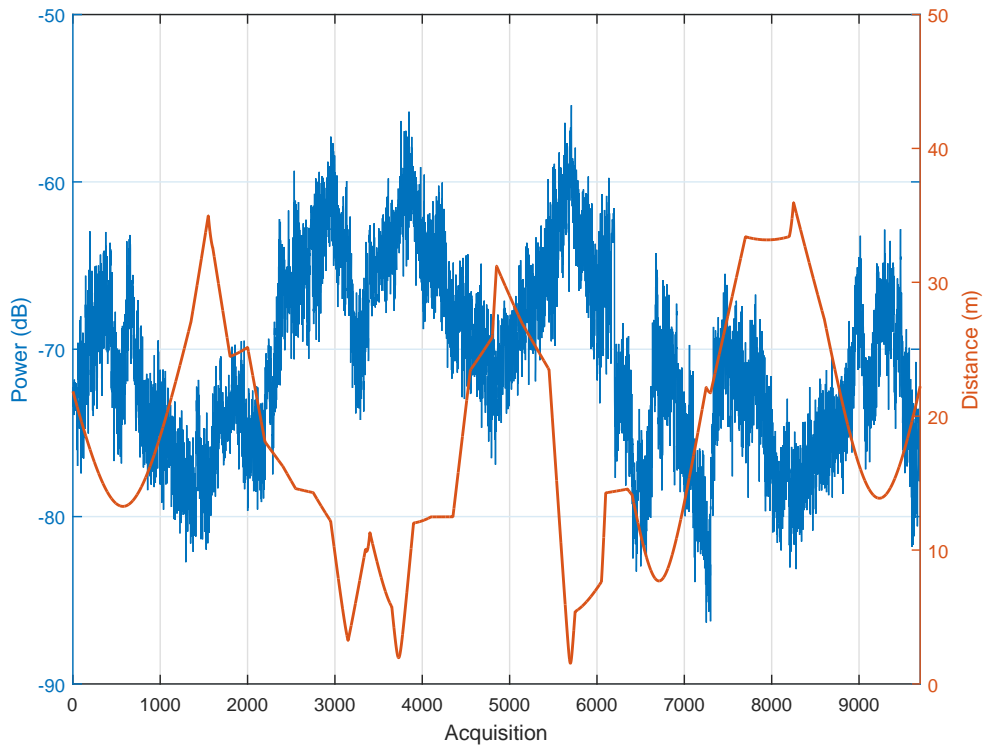

Figure 138: Overall Received Power vs. Acquisition Number and Distance vs. Acquisition Number: Vert Polarization, 5.4GHz, T2, Low.

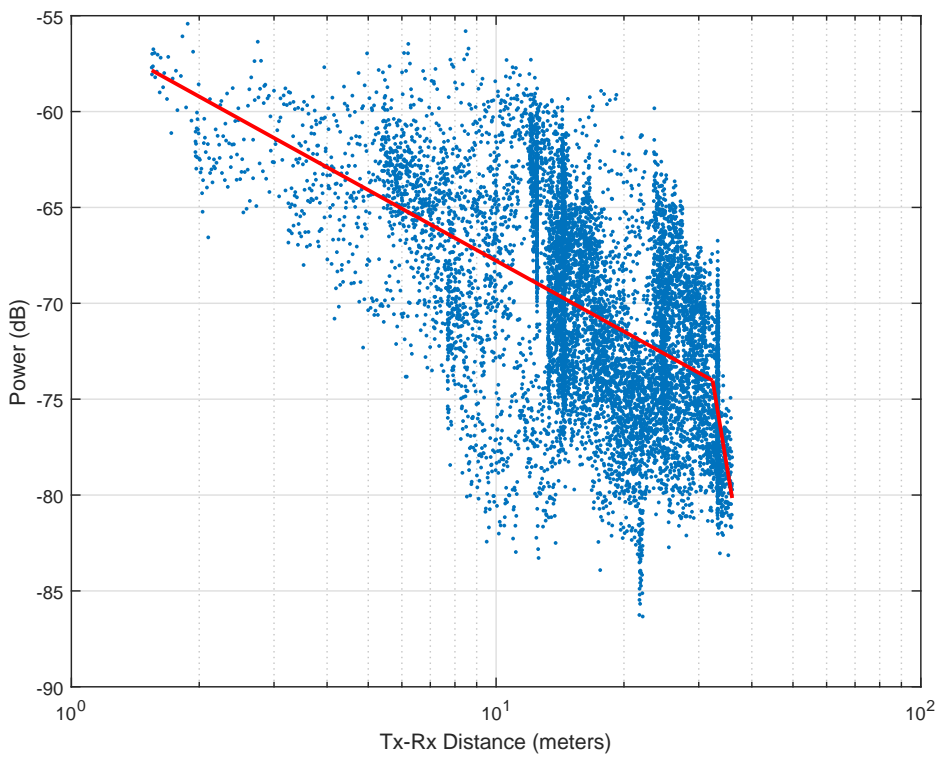

Figure 139: Overall Received Power vs. Distance: Vert Polarization, 5.4GHz, T2, Low. 


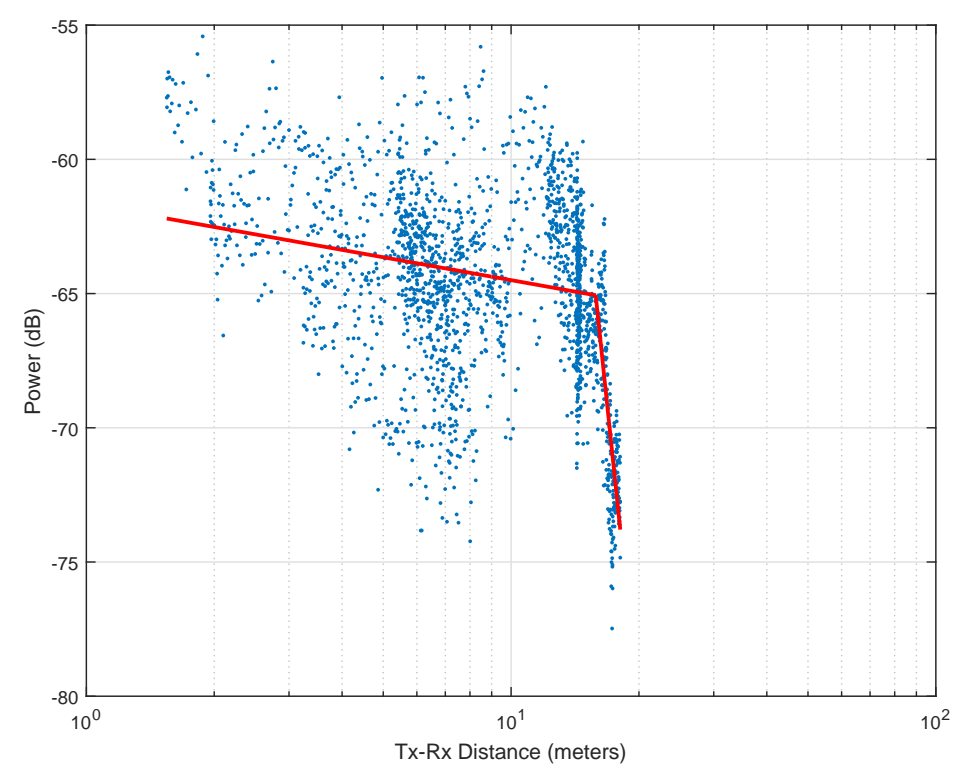

Figure 140: Line of Sight Received Power vs. Distance: Vert Polarization, 5.4GHz, T2, Low.

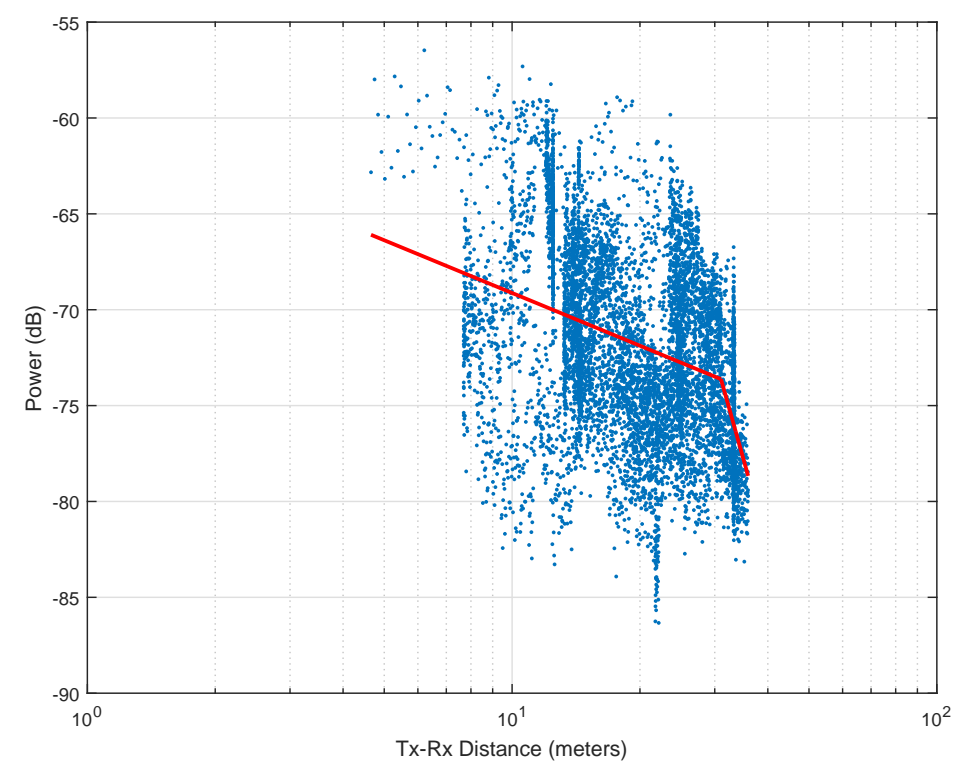

Figure 141: Non Line of Sight Received Power vs. Distance: Vert Polarization, 5.4GHz, T2, Low. 


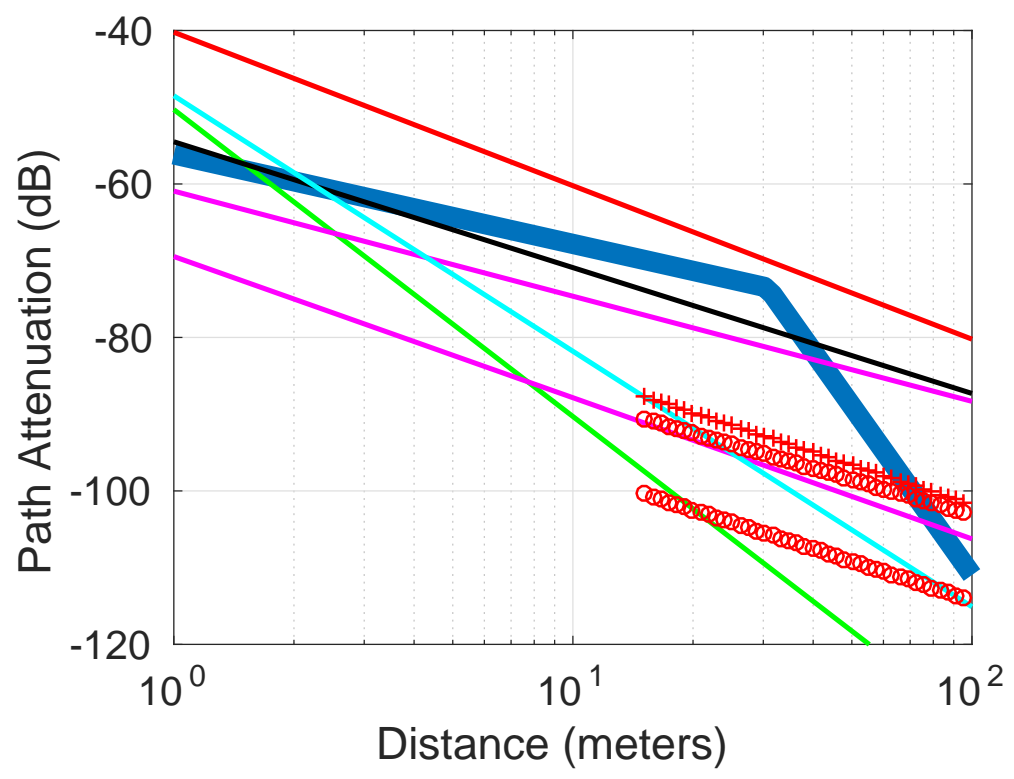

Figure 142: Comparison to other models: Vert Polarization, 5.4GHz, T2, Low.

\begin{tabular}{c|c|c|c|c|c|c|c|c}
\hline Type & Frequency & Polarization & $\alpha_{1}$ & $\alpha_{2}$ & $\beta(\mathrm{m})$ & $k_{1}(\mathrm{~dB})$ & $k_{2}(\mathrm{~dB})$ & MSE \\
\hline \hline Overall & $5.4 \mathrm{GHz}$ & Vpol Low & 1.1667 & 7.3162 & 30.7672 & -56.1461 & 35.3642 & 13.1287 \\
\hline LOS & $5.4 \mathrm{GHz}$ & Vpol Low & 0.28326 & 15.0956 & 15.8195 & -61.6676 & 115.9609 & 9.2591 \\
\hline NLOS & $5.4 \mathrm{GHz}$ & LongPol Low & -1.041 & 1.2328 & 12.2267 & -86.38 & -61.657 & 10.6606 \\
\hline
\end{tabular}

Table 19: Parameters for Vert Polarization, 5GHz, T2, Low 


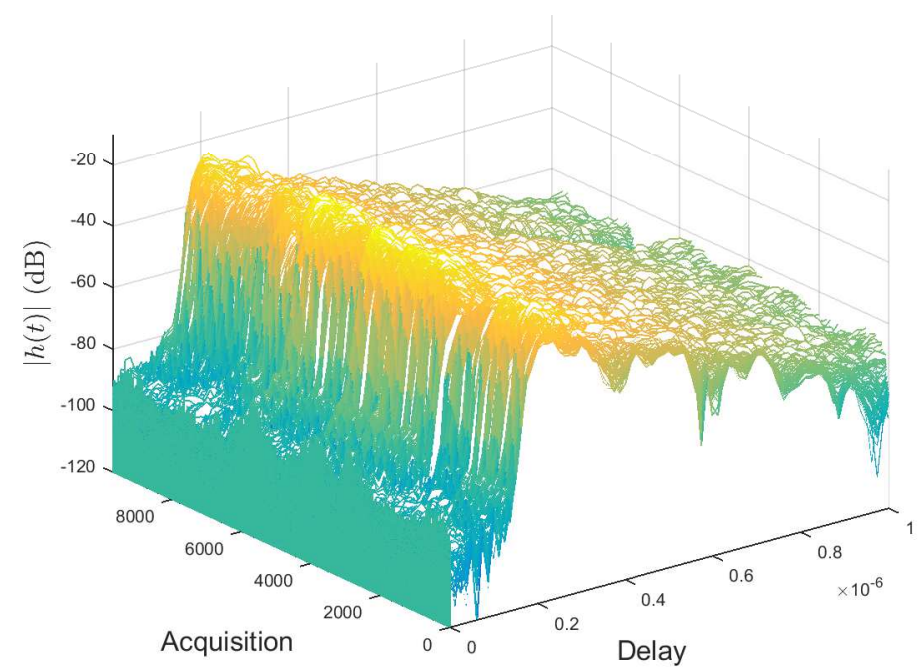

Figure 143: Impulse Responses: Vert Polarization, 5.4GHz, T2, Low.

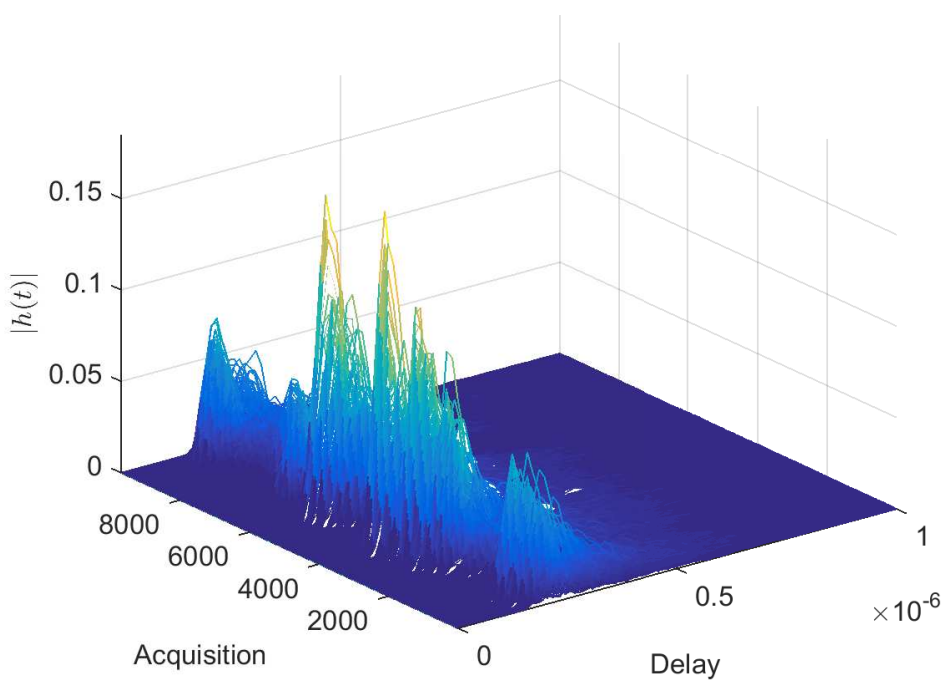

Figure 144: Impulse Responses: Vert Polarization, 5.4GHz, T2, Low. 


\section{A.19 Channel Parameters Summary}

\begin{tabular}{c|c|c|c|c|c|c|c}
\hline Frequency & Polarization & $\alpha_{1}$ & $\alpha_{2}$ & $\beta(\mathrm{m})$ & $k_{1}(\mathrm{~dB})$ & $k_{2}(\mathrm{~dB})$ & MSE \\
\hline $2.245 \mathrm{GHz}$ & Vpol & 0.19369 & 2.7212 & 8.9123 & -53.4288 & -29.4182 & 12.1805 \\
\hline $2.245 \mathrm{GHz}$ & LongPol & -0.15432 & 2.1088 & 8.1815 & -62.1796 & -41.5209 & 9.0812 \\
\hline $2.245 \mathrm{GHz}$ & CrossPol & -0.47776 & 2.166 & 8.4098 & -64.9408 & -40.4919 & 9.3129 \\
\hline $5.4 \mathrm{GHz}$ & Vpol & -0.22296 & 2.6873 & 10.1526 & -65.5349 & -36.2409 & 12.0084 \\
\hline $5.4 \mathrm{GHz}$ & LongPol & 0.86433 & 3.0744 & 19.5126 & -64.6758 & -36.1586 & 9.101 \\
\hline $5.4 \mathrm{GHz}$ & CrossPol & -0.0035534 & 2.2885 & 10.5681 & -71.6171 & -48.1464 & 9.8974 \\
\hline
\end{tabular}

Table 20: Transmitter 1: Overall

\begin{tabular}{c|c|c|c|c|c|c|c}
\hline Frequency & Polarization & $\alpha_{1}$ & $\alpha_{2}$ & $\beta(\mathrm{m})$ & $k_{1}(\mathrm{~dB})$ & $k_{2}(\mathrm{~dB})$ & MSE \\
\hline $2.245 \mathrm{GHz}$ & Vpol & 0.22358 & 1.6849 & 8.8277 & -53.2983 & -39.4768 & 11.0969 \\
\hline $2.245 \mathrm{GHz}$ & LongPol & -0.13092 & 1.8939 & 8.0598 & -62.0107 & -43.6595 & 9.8289 \\
\hline $2.245 \mathrm{GHz}$ & CrossPol & -0.48848 & 1.6744 & 7.8537 & -64.8572 & -45.4973 & 9.5928 \\
\hline $5.4 \mathrm{GHz}$ & Vpol & -0.24922 & 1.5547 & 8.8181 & -65.6198 & -48.566 & 10.1366 \\
\hline $5.4 \mathrm{GHz}$ & LongPol & 0.3571 & 2.0845 & 13.2858 & -68.4398 & -49.0348 & 9.2727 \\
\hline $5.4 \mathrm{GHz}$ & CrossPol & -0.20753 & 1.5937 & 8.1212 & -72.7759 & -56.3919 & 10.1328 \\
\hline
\end{tabular}

Table 21: Transmitter 1: Line of Sight

\begin{tabular}{c|c|c|c|c|c|c|c}
\hline Frequency & Polarization & $\alpha_{1}$ & $\alpha_{2}$ & $\beta(\mathrm{m})$ & $k_{1}(\mathrm{~dB})$ & $k_{2}(\mathrm{~dB})$ & MSE \\
\hline $2.245 \mathrm{GHz}$ & Vpol & 2.6179 & 7.3565 & 31.9439 & -31.3789 & 39.9079 & 10.1757 \\
\hline $2.245 \mathrm{GHz}$ & LongPol & 1.4813 & 2.8369 & 20.537 & -49.1724 & -31.379 & 8.2535 \\
\hline $2.245 \mathrm{GHz}$ & CrossPol & 1.4082 & 3.3221 & 20.3506 & -49.7419 & -24.6974 & 8.0049 \\
\hline $5.4 \mathrm{GHz}$ & Vpol & 1.6346 & 4.5464 & 21.8949 & -49.2994 & -10.2715 & 10.9476 \\
\hline $5.4 \mathrm{GHz}$ & LongPol & 1.1325 & 3.4085 & 20.2497 & -61.7334 & -31.9992 & 8.2512 \\
\hline $5.4 \mathrm{GHz}$ & CrossPol & 1.3306 & 3.3114 & 19.5742 & -59.6536 & -34.0677 & 8.8339 \\
\hline
\end{tabular}

Table 22: Transmitter 1: Non Line of Sight 


\begin{tabular}{c|c|c|c|c|c|c|c}
\hline Bandwidth & Polarization & $\alpha_{1}$ & $\alpha_{2}$ & $\beta(\mathrm{m})$ & $k_{1}(\mathrm{~dB})$ & $k_{2}(\mathrm{~dB})$ & MSE \\
\hline $2.245 \mathrm{GHz}$ & Vpol Low & 1.0029 & 2.0598 & 12.1722 & -49.9412 & -38.4701 & 12.8307 \\
\hline $2.245 \mathrm{GHz}$ & LongPol Low & -1.1956 & 1.4134 & 10.2097 & -76.5594 & -50.2345 & 12.093 \\
\hline $2.245 \mathrm{GHz}$ & CrossPol Low & 1.251 & 3.4239 & 28.266 & -52.6428 & -21.1079 & 10.4894 \\
\hline $2.245 \mathrm{GHz}$ & Vpol High & -0.24426 & 1.5046 & 6.4786 & -59.4669 & -45.2756 & 12.1637 \\
\hline $2.245 \mathrm{GHz}$ & LongPol High & 0.84922 & 1.6614 & 12.4132 & -55.9006 & -47.0167 & 10.8449 \\
\hline $2.245 \mathrm{GHz}$ & CrossPol High & 1.1065 & 4.3014 & 28.4304 & -54.2346 & -7.7875 & 10.2871 \\
\hline $5.4 \mathrm{GHz}$ & Vpol Low & 1.1667 & 7.3162 & 30.7672 & -56.1461 & 35.3642 & 13.1287 \\
\hline $5.4 \mathrm{GHz}$ & LongPol Low & 0.79698 & 1.3677 & 14.4068 & -66.2022 & -59.5904 & 11.1193 \\
\hline $5.4 \mathrm{GHz}$ & CrossPol Low & 0.8628 & 5.5696 & 29.8334 & -66.2216 & 3.1899 & 11.5172 \\
\hline $5.4 \mathrm{GHz}$ & Vpol High & 0.84074 & 3.8845 & 30.0082 & -60.6292 & -15.665 & 12.4382 \\
\hline $5.4 \mathrm{GHz}$ & LongPol High & 0.90942 & 6.478 & 30.9429 & -65.0881 & 17.9146 & 11.5051 \\
\hline $5.4 \mathrm{GHz}$ & CrossPol High & 0.86394 & 5.9326 & 30.3528 & -65.7543 & 9.3732 & 11.326 \\
\hline
\end{tabular}

Table 23: Transmitter 2: Overall 


\begin{tabular}{c|c|c|c|c|c|c|c}
\hline Frequency & Polarization & $\alpha_{1}$ & $\alpha_{2}$ & $\beta(\mathrm{m})$ & $k_{1}(\mathrm{~dB})$ & $k_{2}(\mathrm{~dB})$ & MSE \\
\hline $2.245 \mathrm{GHz}$ & Vpol Low & 0.21386 & 10.171 & 13.9837 & -54.3781 & 59.6931 & 10.7806 \\
\hline $2.245 \mathrm{GHz}$ & LongPol Low & -1.557 & 7.0381 & 13.3428 & -78.3206 & 18.3958 & 11.2471 \\
\hline $2.245 \mathrm{GHz}$ & CrossPol Low & 0.22769 & 7.2461 & 13.7639 & -59.7015 & 20.2204 & 8.9558 \\
\hline $2.245 \mathrm{GHz}$ & Vpol High & 0.0012037 & 8.1405 & 13.6994 & -57.222 & 35.298 & 8.0396 \\
\hline $2.245 \mathrm{GHz}$ & LongPol High & 0.060714 & 9.0037 & 14.1156 & -60.5855 & 42.2322 & 8.2734 \\
\hline $2.245 \mathrm{GHz}$ & CrossPol High & 0.048162 & 7.1472 & 13.3019 & -61.6634 & 18.1234 & 7.6617 \\
\hline $5.4 \mathrm{GHz}$ & Vpol Low & 0.28326 & 15.0956 & 15.8195 & -61.6676 & 115.9609 & 9.2591 \\
\hline $5.4 \mathrm{GHz}$ & LongPol Low & 0.19605 & 7.6672 & 14.3712 & -69.4231 & 17.0553 & 8.5566 \\
\hline $5.4 \mathrm{GHz}$ & CrossPol Low & 0.18635 & 5.8664 & 13.743 & -69.8559 & -5.2128 & 8.6103 \\
\hline $5.4 \mathrm{GHz}$ & Vpol High & 0.1585 & 3.9643 & 13.4396 & -64.6383 & -21.6947 & 8.3161 \\
\hline $5.4 \mathrm{GHz}$ & LongPol High & 0.17308 & 6.3922 & 13.8303 & -69.4213 & 1.5281 & 8.4983 \\
\hline $5.4 \mathrm{GHz}$ & CrossPol High & 0.19992 & 5.0439 & 13.5367 & -69.3439 & -14.534 & 7.6552 \\
\hline
\end{tabular}

Table 24: Transmitter 2: Line of Sight 


\begin{tabular}{c|c|c|c|c|c|c|c}
\hline Frequency & Polarization & $\alpha_{1}$ & $\alpha_{2}$ & $\beta(\mathrm{m})$ & $k_{1}(\mathrm{~dB})$ & $k_{2}(\mathrm{~dB})$ & MSE \\
\hline $2.245 \mathrm{GHz}$ & Vpol Low & -0.36991 & 1.8857 & 12.0498 & -65.4945 & -41.1119 & 12.2427 \\
\hline $2.245 \mathrm{GHz}$ & LongPol Low & -1.7714 & 1.1321 & 12.4823 & -86.108 & -54.2775 & 11.4781 \\
\hline $2.245 \mathrm{GHz}$ & CrossPol Low & -0.86565 & 1.5089 & 12.0315 & -75.3644 & -49.7118 & 10.0073 \\
\hline $2.245 \mathrm{GHz}$ & LongPol High & -1.1611 & 1.5052 & 12.0615 & -78.2653 & -49.4317 & 9.8642 \\
\hline $2.245 \mathrm{GHz}$ & CrossPol High & -1.5062 & 1.5834 & 12.0752 & -81.815 & -48.3886 & 9.8115 \\
\hline $5.4 \mathrm{GHz}$ & Vpol Low & 0.91366 & 7.8889 & 31.0319 & -59.991 & 44.0663 & 12.9751 \\
\hline $5.4 \mathrm{GHz}$ & LongPol Low & -1.041 & 1.2328 & 12.2267 & -86.38 & -61.657 & 10.6606 \\
\hline $5.4 \mathrm{GHz}$ & CrossPol Low & -1.8951 & 1.1466 & 12.1666 & -96.0213 & -63.0146 & 11.1937 \\
\hline $5.4 \mathrm{GHz}$ & Vpol High & -0.87168 & 0.99421 & 12.4772 & -79.5216 & -59.0693 & 12.5158 \\
\hline $5.4 \mathrm{GHz}$ & LongPol High & -1.3559 & 1.0987 & 12.1978 & -89.6896 & -63.0264 & 11.156 \\
\hline $5.4 \mathrm{GHz}$ & CrossPol High & 0.34127 & 5.1472 & 28.8608 & -72.859 & -2.6781 & 11.0662 \\
\hline
\end{tabular}

Table 25: Transmitter 2: Non Line of Sight 


\begin{tabular}{c|c|c|c|c}
\hline Frequency & TX & Polarization & RMS Delay Spread & K-factor \\
\hline $2.245 \mathrm{GHz}$ & 1 & Vpol & $2.8422 \mathrm{e}-07$ & 0.49409 \\
$2.245 \mathrm{GHz}$ & 1 & CrossPol & $3.2197 \mathrm{e}-07$ & 0.19126 \\
$2.245 \mathrm{GHz}$ & 1 & LongPol & $4.1112 \mathrm{e}-07$ & 0.25174 \\
$5.4 \mathrm{GHz}$ & 1 & Vpol & $9.3043 \mathrm{e}-08$ & 0.68195 \\
$5.4 \mathrm{GHz}$ & 1 & CrossPol & $9.7013 \mathrm{e}-08$ & 0.33833 \\
$5.4 \mathrm{GHz}$ & 1 & LongPol & $1.0014 \mathrm{e}-07$ & 0.30412 \\
$5.4 \mathrm{GHz}$ & 2 low & Vpol & $1.0305 \mathrm{e}-07$ & 0.56598 \\
$5.4 \mathrm{GHz}$ & 2 low & CrossPol & $4.3927 \mathrm{e}-06$ & 0.32378 \\
$5.4 \mathrm{GHz}$ & 2 low & LongPol & $2.3537 \mathrm{e}-05$ & 0.29494 \\
$5.4 \mathrm{GHz}$ & 2 high & Vpol & $3.1401 \mathrm{e}-07$ & 0.74183 \\
$5.4 \mathrm{GHz}$ & 2 high & CrossPol & $3.3235 \mathrm{e}-07$ & 0.21757 \\
$5.4 \mathrm{GHz}$ & 2 high & LongPol & $4.7888 \mathrm{e}-07$ & 0.24105 \\
$2.245 \mathrm{GHz}$ & 2 low & Vpol & $2.7475 \mathrm{e}-07$ & 0.59979 \\
$2.245 \mathrm{GHz}$ & 2 low & CrossPol & $1.1766 \mathrm{e}-07$ & 0.34546 \\
$2.245 \mathrm{GHz}$ & 2 low & LongPol & $1.0659 \mathrm{e}-07$ & 0.36303 \\
$2.245 \mathrm{GHz}$ & 2high & Vpol & $8.9047 \mathrm{e}-08$ & 0.46737 \\
$2.245 \mathrm{GHz}$ & 2 high & CrossPol & $1.0123 \mathrm{e}-07$ & 0.26226 \\
$2.245 \mathrm{GHz}$ & 2 high & LongPol & $1.0198 \mathrm{e}-07$ & 0.24948
\end{tabular}




\section{A.20 2.245GHz, Transmitter 1}

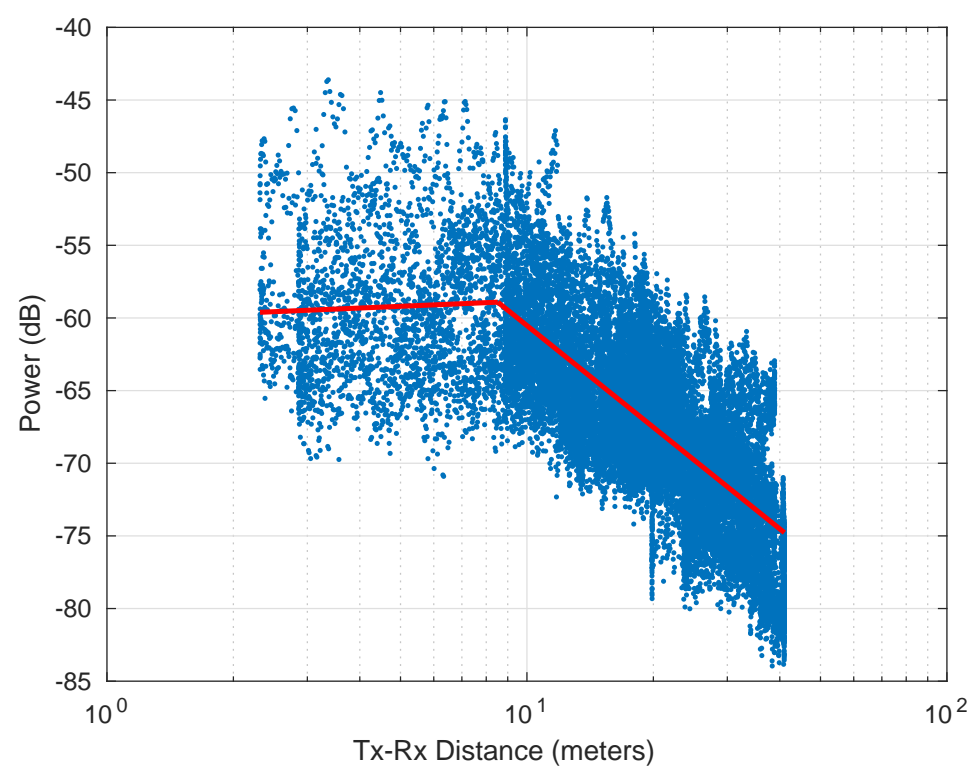

Figure 145: Overall Received Power vs. Distance: All Polarizations, 2.245GHz, T1.

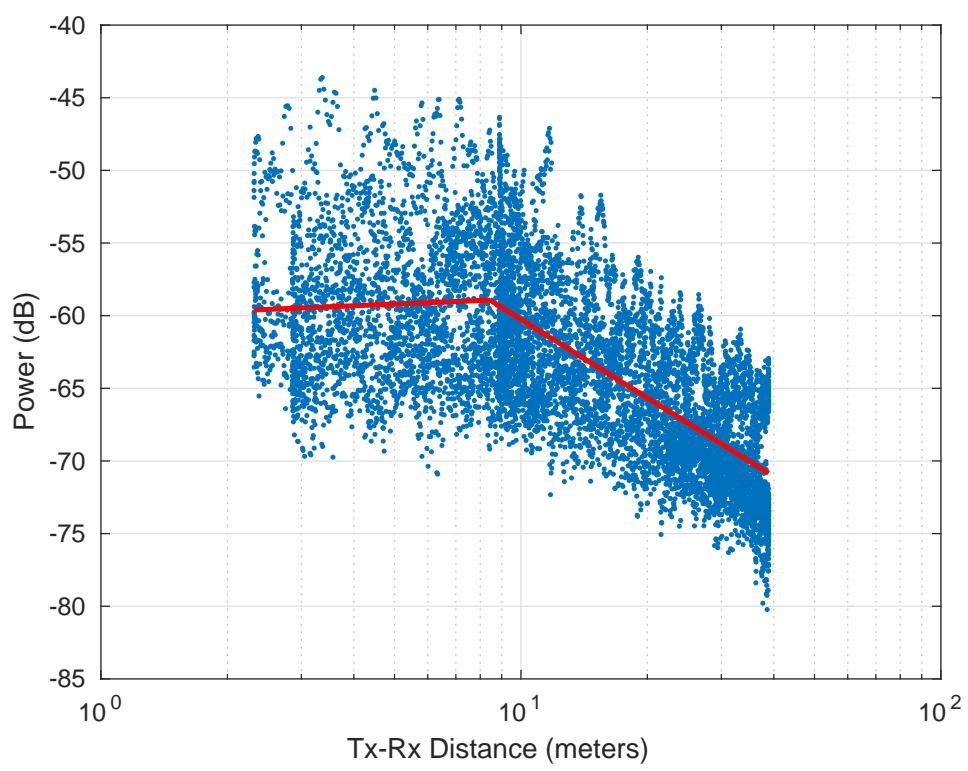

Figure 146: Line of Sight Received Power vs. Distance: All Polarizations, 2.245GHz, T1. 


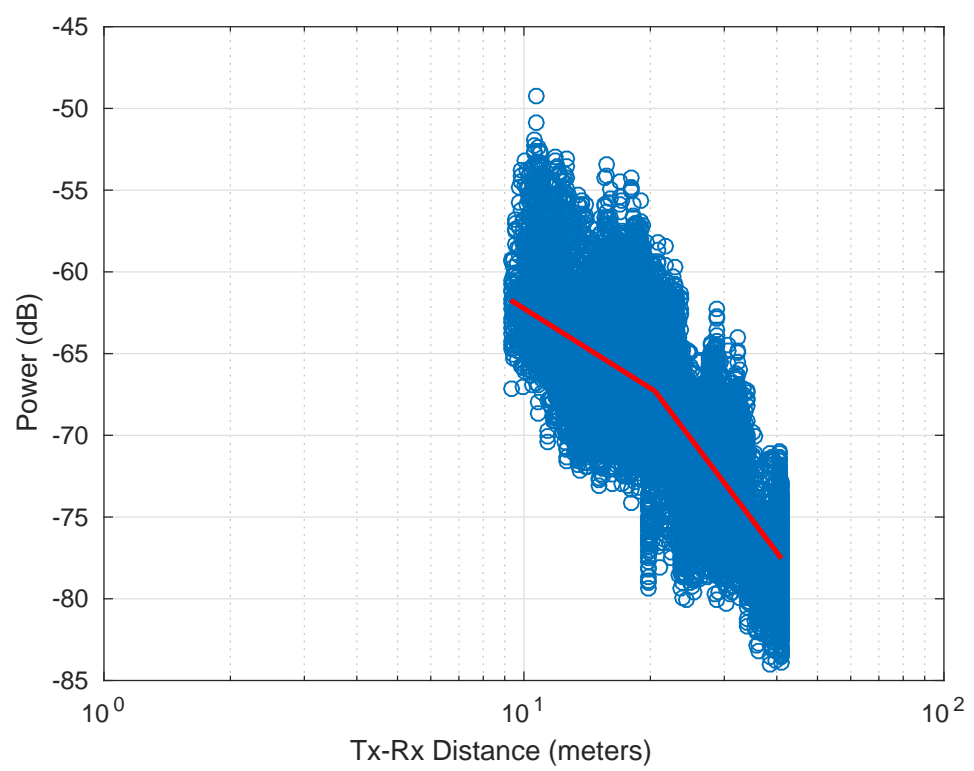

Figure 147: Non Line of Sight Received Power vs. Distance: All Polarizations, 2.245GHz, $\mathrm{T} 1$.

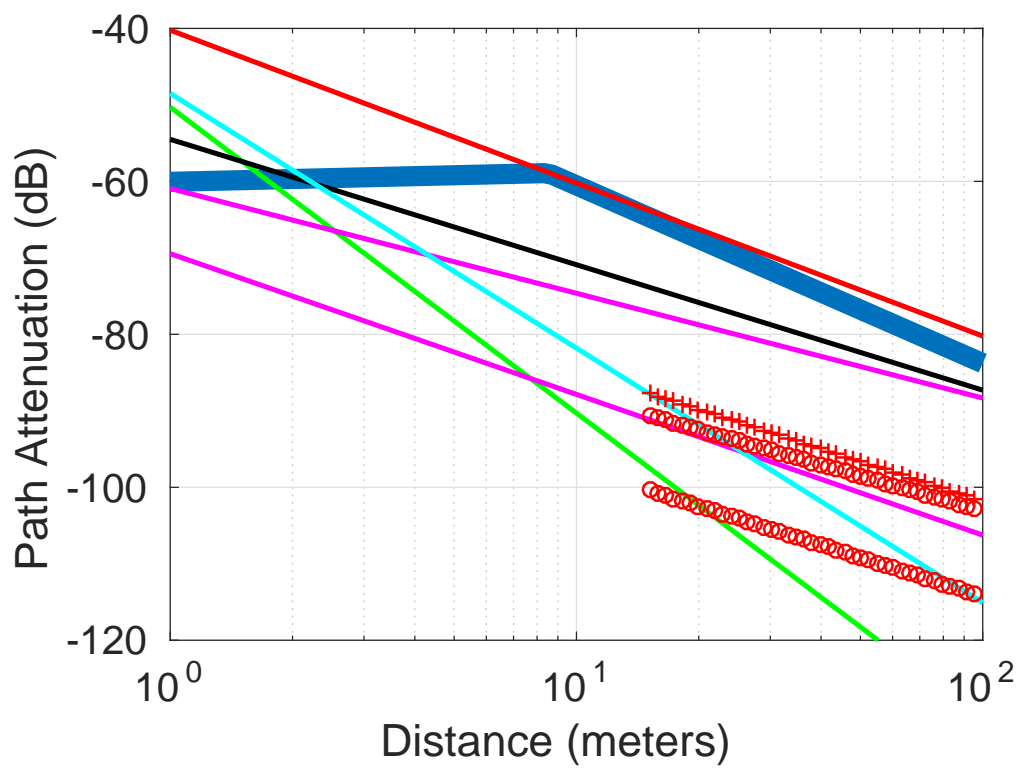

Figure 148: Comparison to other models: All Polarizations, 2.245GHz, T1.

\begin{tabular}{c|c|c|c|c|c}
\hline$\alpha_{1}$ & $\alpha_{2}$ & $\beta(\mathrm{m})$ & $k_{1}(\mathrm{~dB})$ & $k_{2}(\mathrm{~dB})$ & $\mathrm{MSE}$ \\
\hline-0.12327 & 2.3246 & 8.524 & -60.0594 & -37.2783 & 11.8458
\end{tabular}

Table 26: Parameters for All Polarizations, 2.245GHz, T1 


\section{A.21 5.4GHz, Transmitter 1}

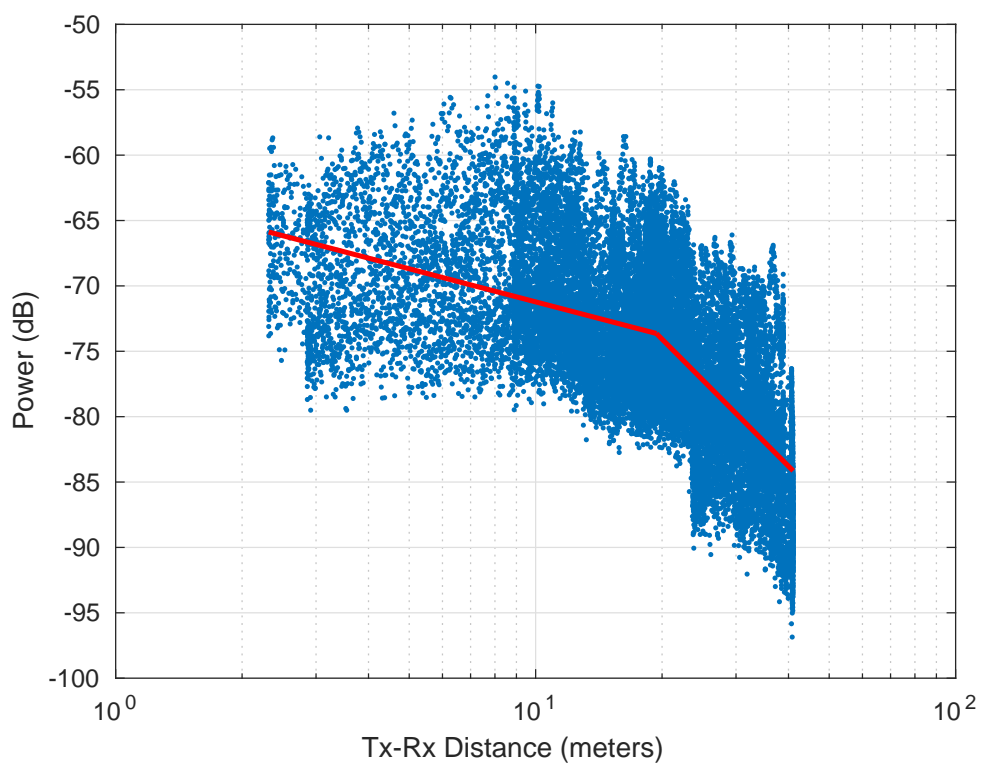

Figure 149: Overall Received Power vs. Distance: All Polarizations, 5.4GHz, T1.

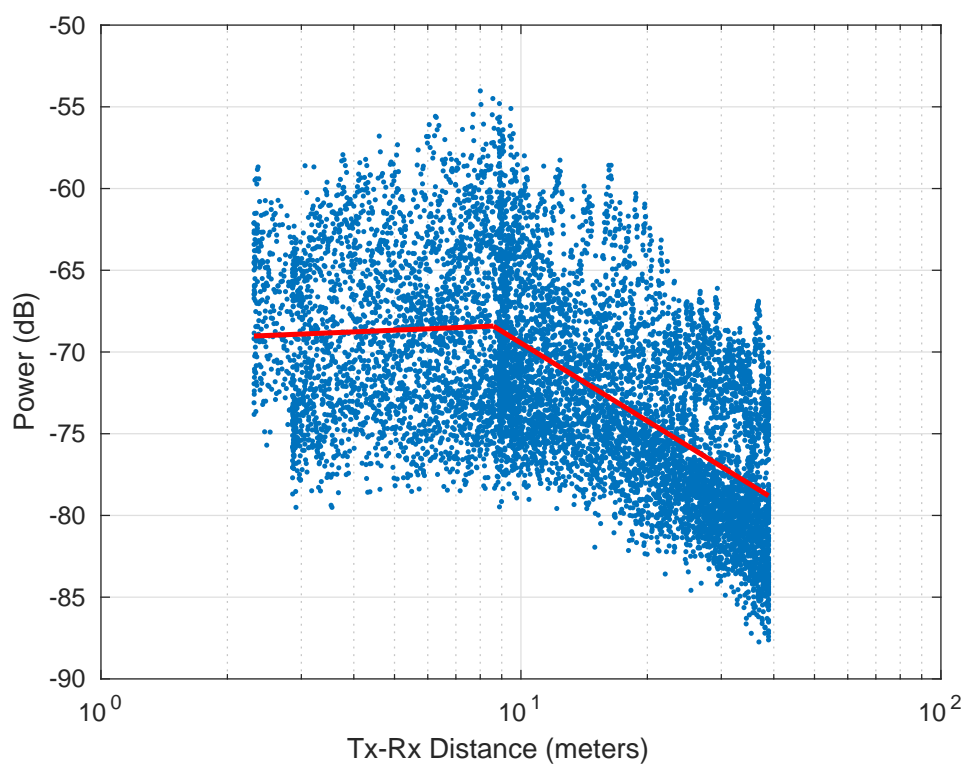

Figure 150: Line of Sight Received Power vs. Distance: All Polarizations, 5.4GHz, T1. 


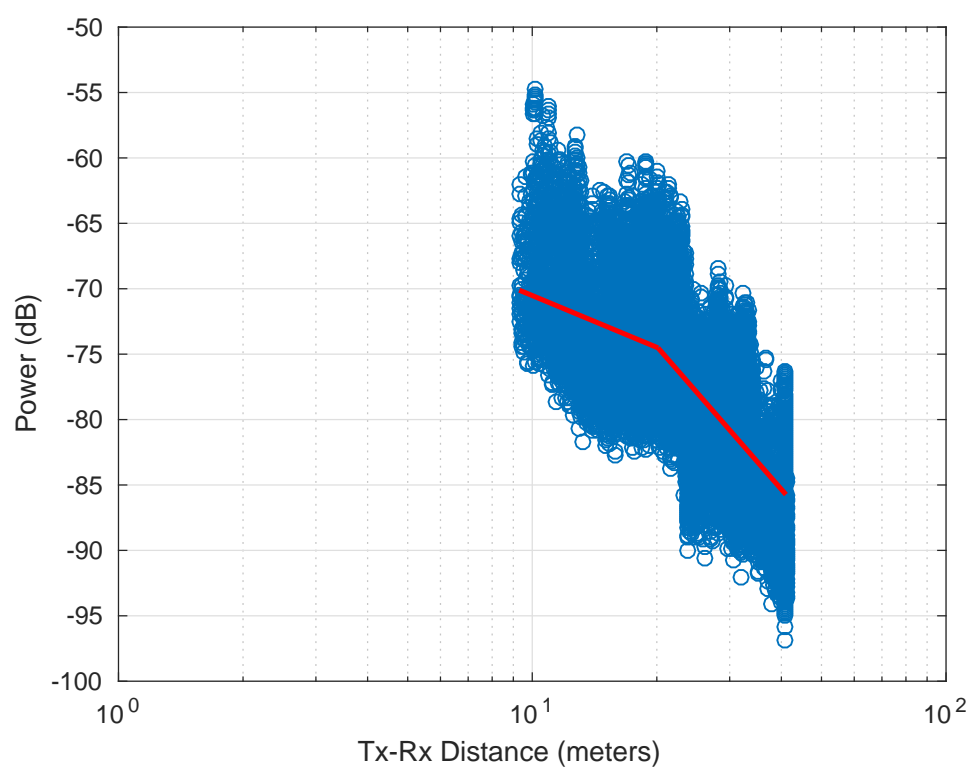

Figure 151: Non Line of Sight Received Power vs. Distance: All Polarizations, 5.4GHz, $\mathrm{T} 1$.

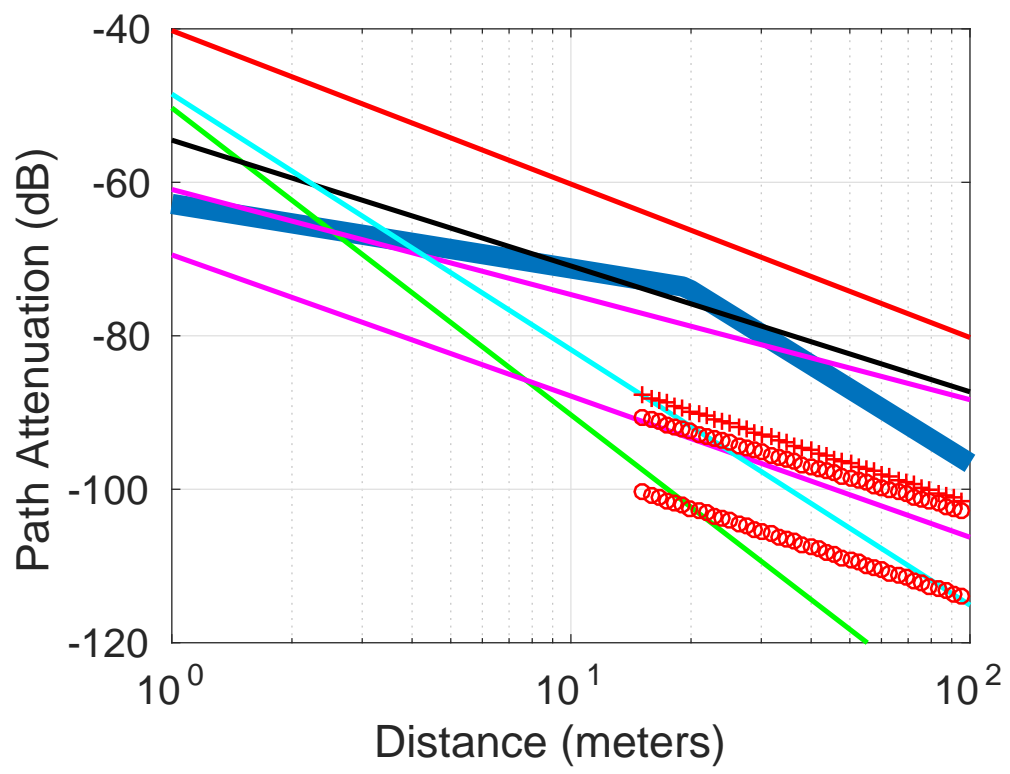

Figure 152: Comparison to other models: All Polarizations, 5.4GHz, T1.

\begin{tabular}{c|c|c|c|c|c}
\hline$\alpha_{1}$ & $\alpha_{2}$ & $\beta(\mathrm{m})$ & $k_{1}(\mathrm{~dB})$ & $k_{2}(\mathrm{~dB})$ & $\mathrm{MSE}$ \\
\hline 0.64026 & 1.6489 & 12.4705 & -57.1717 & -46.1186 & 12.7361
\end{tabular}

Table 27: Parameters for All Polarizations, 5.4GHz, T1 


\section{A.22 2.245GHz, Transmitter 2}

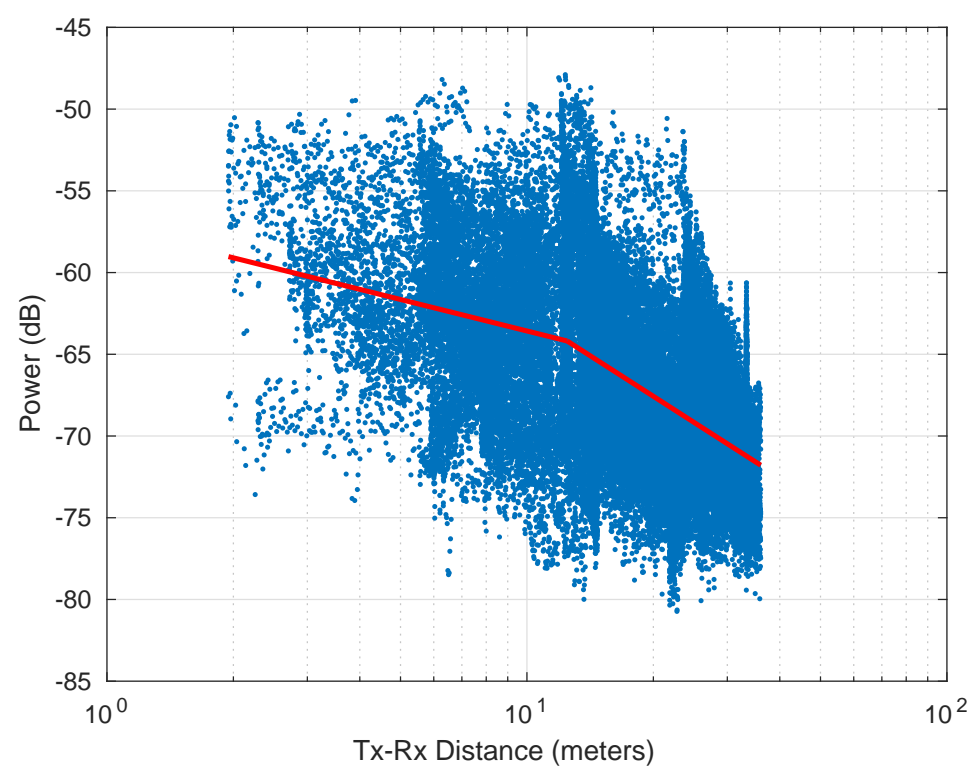

Figure 153: Overall Received Power vs. Distance: All Polarizations, 2.245GHz, T2.

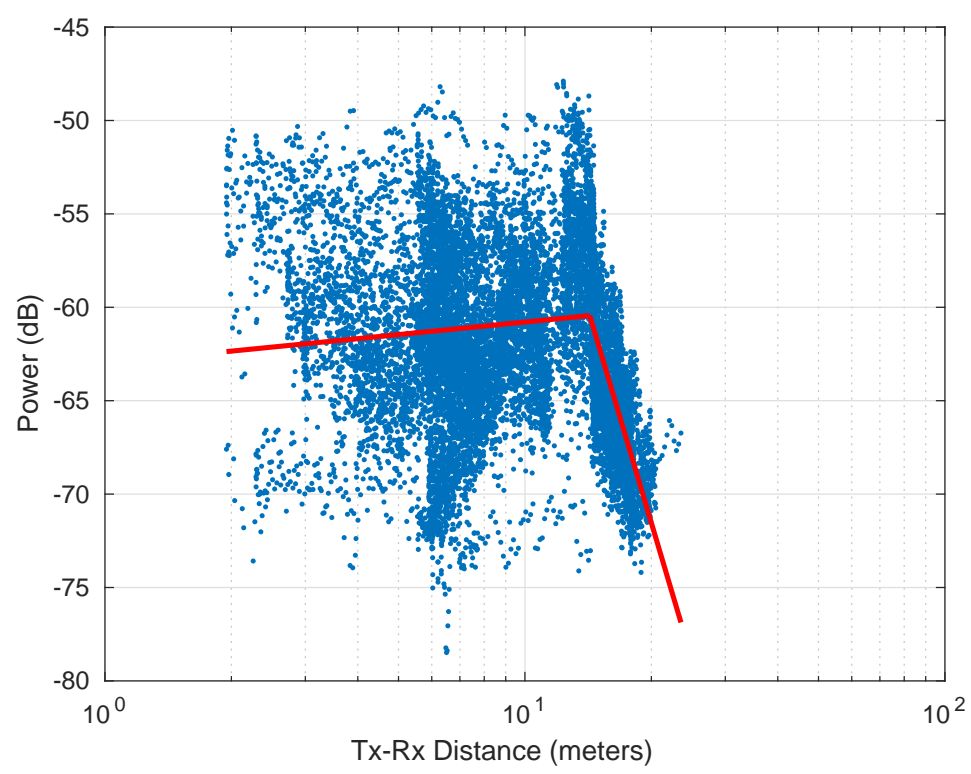

Figure 154: Line of Sight Received Power vs. Distance: All Polarizations, 2.245GHz, T2. 


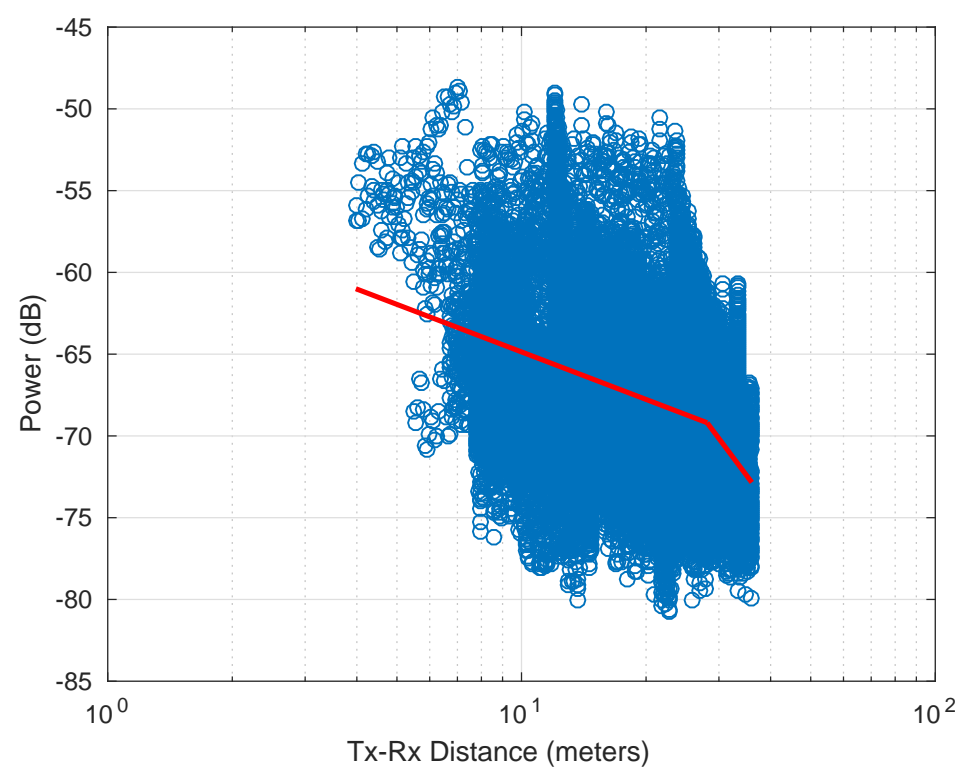

Figure 155: Non Line of Sight Received Power vs. Distance: All Polarizations, 2.245GHz, $\mathrm{T} 2$.

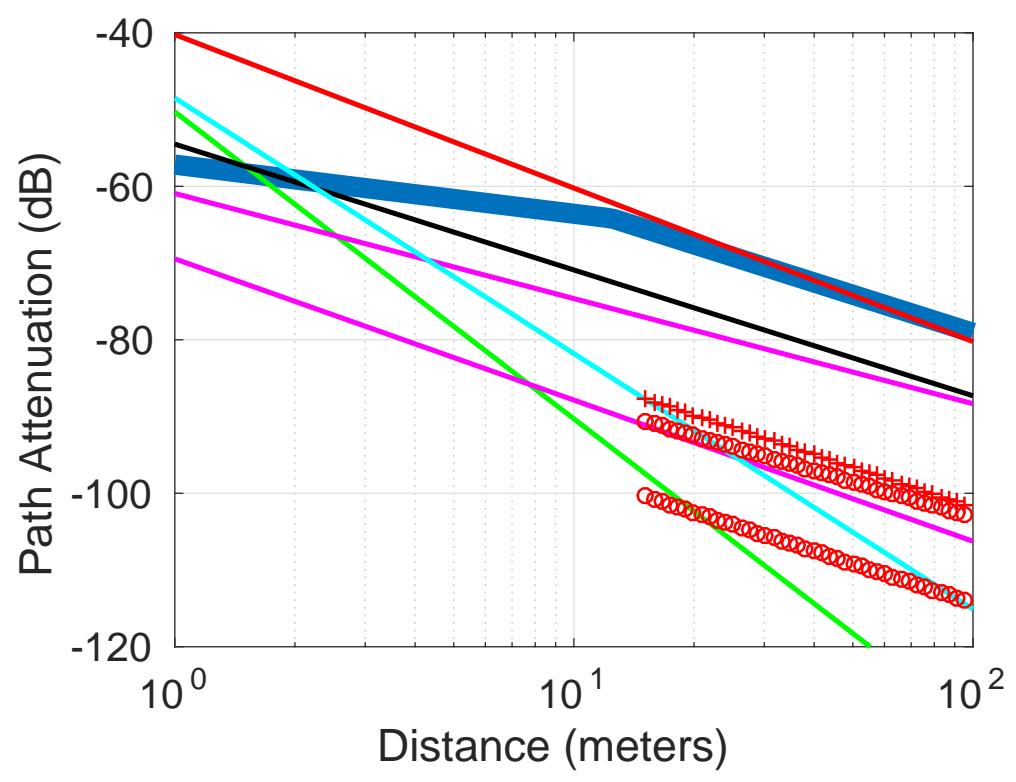

Figure 156: Comparison to other models: All Polarizations, 2.245GHz, T2.

\begin{tabular}{c|c|c|c|c|c}
\hline$\alpha_{1}$ & $\alpha_{2}$ & $\beta(\mathrm{m})$ & $k_{1}(\mathrm{~dB})$ & $k_{2}(\mathrm{~dB})$ & $\mathrm{MSE}$ \\
\hline 0.84057 & 3.2263 & 19.3861 & -62.8153 & -32.0997 & 13.2548
\end{tabular}

Table 28: Parameters for All Polarizations, 2.245GHz, T2 


\section{A.23 5.4GHz, Transmitter 2}

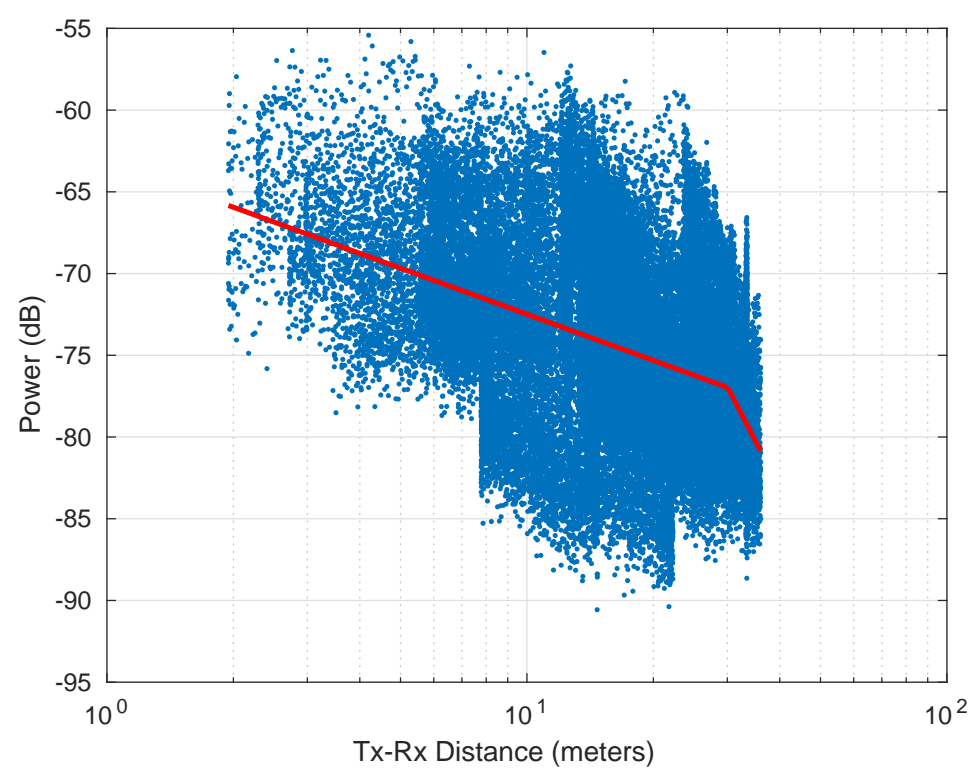

Figure 157: Overall Received Power vs. Distance: All Polarizations, 5.4GHz, T2.

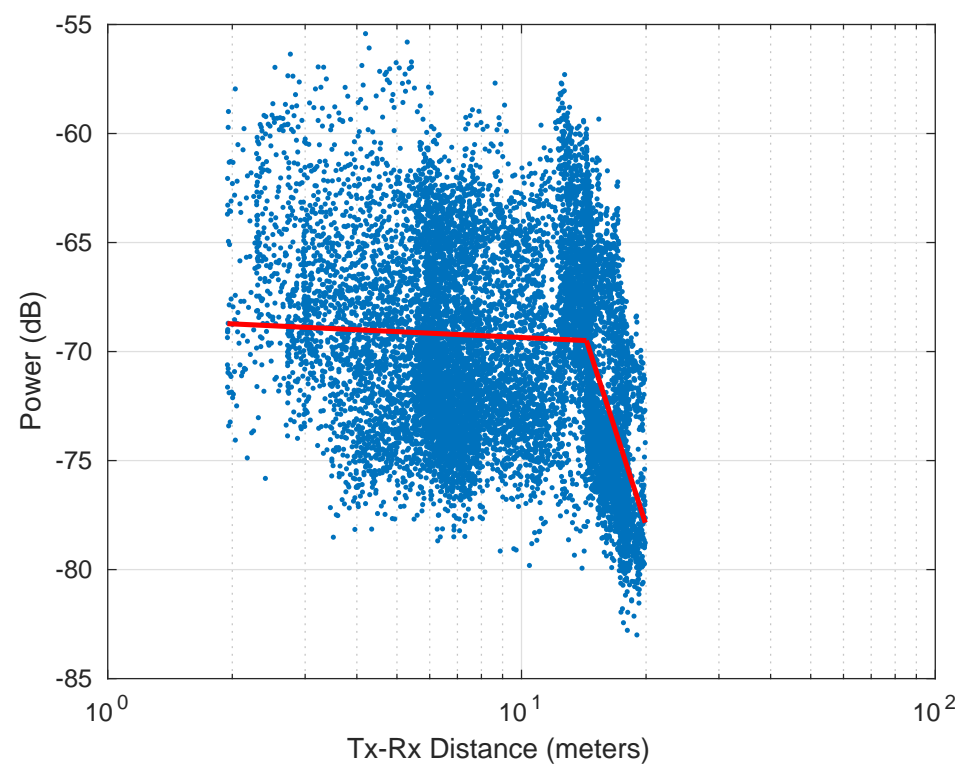

Figure 158: Line of Sight Received Power vs. Distance: All Polarizations, 5.4GHz, T2. 


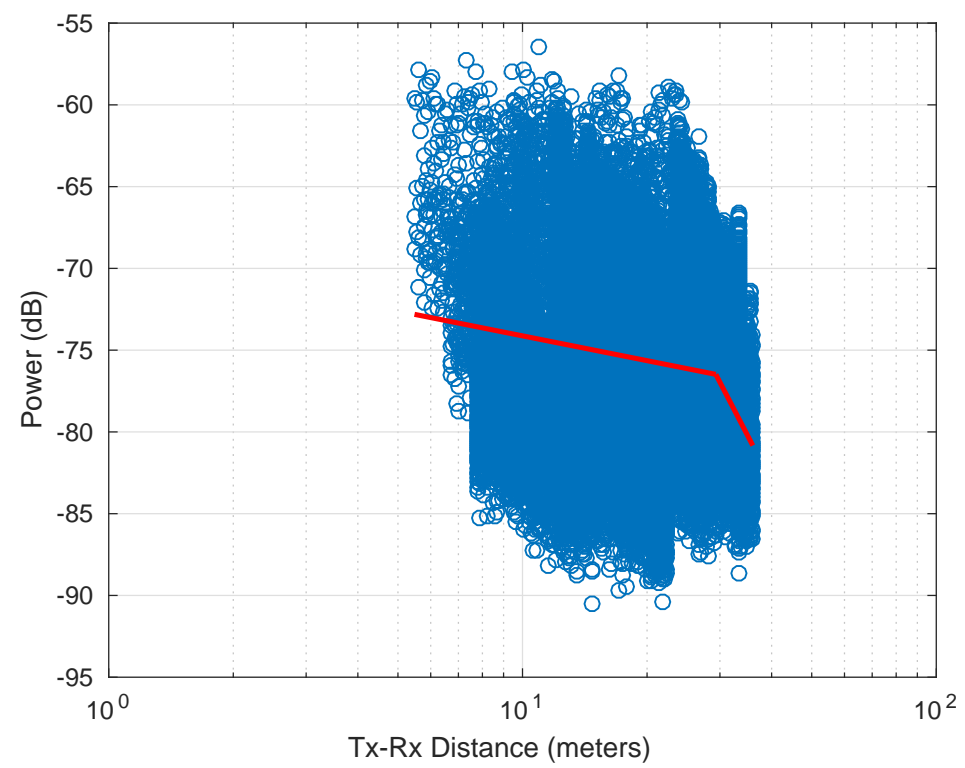

Figure 159: Non Line of Sight Received Power vs. Distance: All Polarizations, 5.4GHz, $\mathrm{T} 2$.

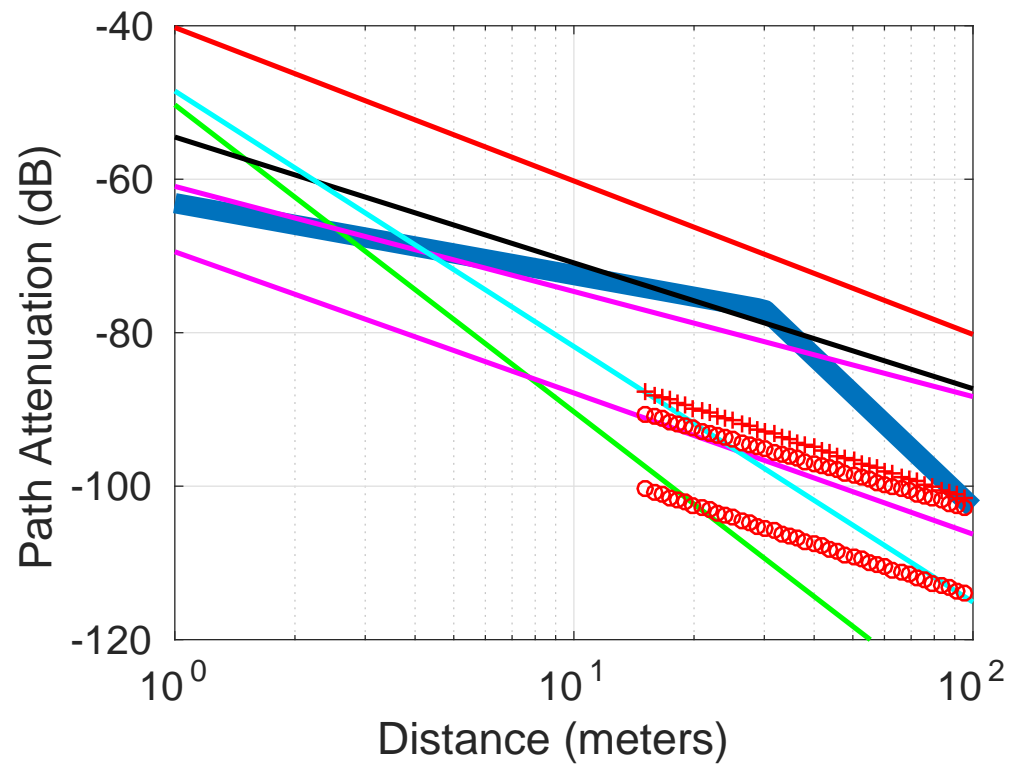

Figure 160: Comparison to other models: All Polarizations, 5.4GHz, T2.

\begin{tabular}{c|c|c|c|c|c|c|c|c}
\hline Location & Frequency & TX & $\alpha_{1}$ & $\alpha_{2}$ & $\beta(\mathrm{m})$ & $k_{1}(\mathrm{~dB})$ & $k_{2}(\mathrm{~dB})$ & MSE \\
\hline GBurg & $2.245 .4 \mathrm{GHz}$ & 1 & -0.12327 & 2.3246 & 8.524 & -60.0594 & -37.2783 & 11.8458 \\
GBurg & $5.4 \mathrm{GHz}$ & 1 & 0.64026 & 1.6489 & 12.4705 & -57.1717 & -46.1186 & 12.7361 \\
GBurg & $2.245 .4 \mathrm{GHz}$ & 2 & 0.84057 & 3.2263 & 19.3861 & -62.8153 & -32.0997 & 13.2548 \\
GBurg & $5.4 \mathrm{GHz}$ & 2 & 0.93732 & 4.948 & 30.0591 & -63.1193 & -3.8426 & 13.6119
\end{tabular}




\begin{tabular}{c|c|c|c|c|c}
\hline$\alpha_{1}$ & $\alpha_{2}$ & $\beta(\mathrm{m})$ & $k_{1}(\mathrm{~dB})$ & $k_{2}(\mathrm{~dB})$ & $\mathrm{MSE}$ \\
\hline 0.93732 & 4.948 & 30.0591 & -63.1193 & -3.8426 & 13.6119
\end{tabular}

Table 29: Parameters for All Polarizations, 5.4GHz, T2 


\section{B Received Power Data: Automotive Assembly}

In this appendix we present the data regarding received power for various measurements in an automotive assembly plant. There were two paths taken, called "inner" and "outer". We have not provided the map of the plant.

\section{B.1 Horizontal Polarization, 2.245GHz, Transmitter 1 Outer Path}

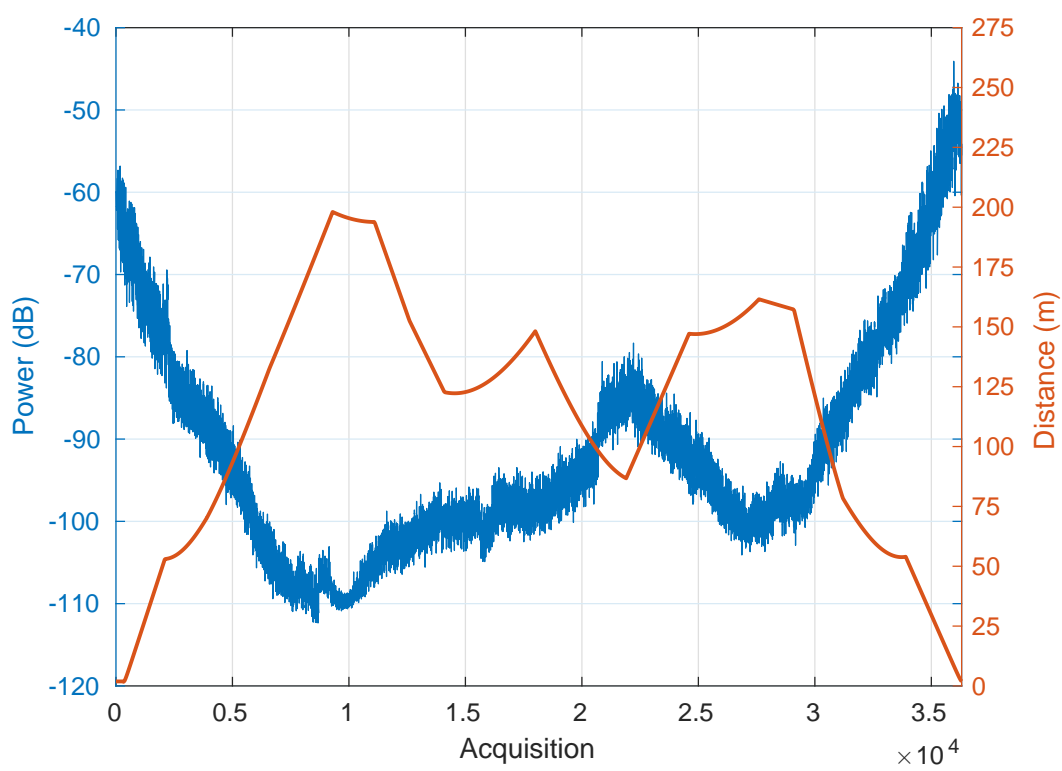

Figure 161: Overall Received Power vs. Acquisition Number and Distance vs. Acquisition Number: Horizontal Polarization, 2.245GHz, T1, Outer Path.

\begin{tabular}{c|c|c|c|c|c}
\hline$\alpha_{1}$ & $\alpha_{2}$ & $\beta(\mathrm{m})$ & $k_{1}(\mathrm{~dB})$ & $k_{2}(\mathrm{~dB})$ & MSE \\
\hline 0.29658 & 5.1625 & 27.5135 & -57.9935 & 12.054 & 13.0546
\end{tabular}

Table 30: Parameters for Horizontal Polarization, 2.245GHz, T1, Outer Path 


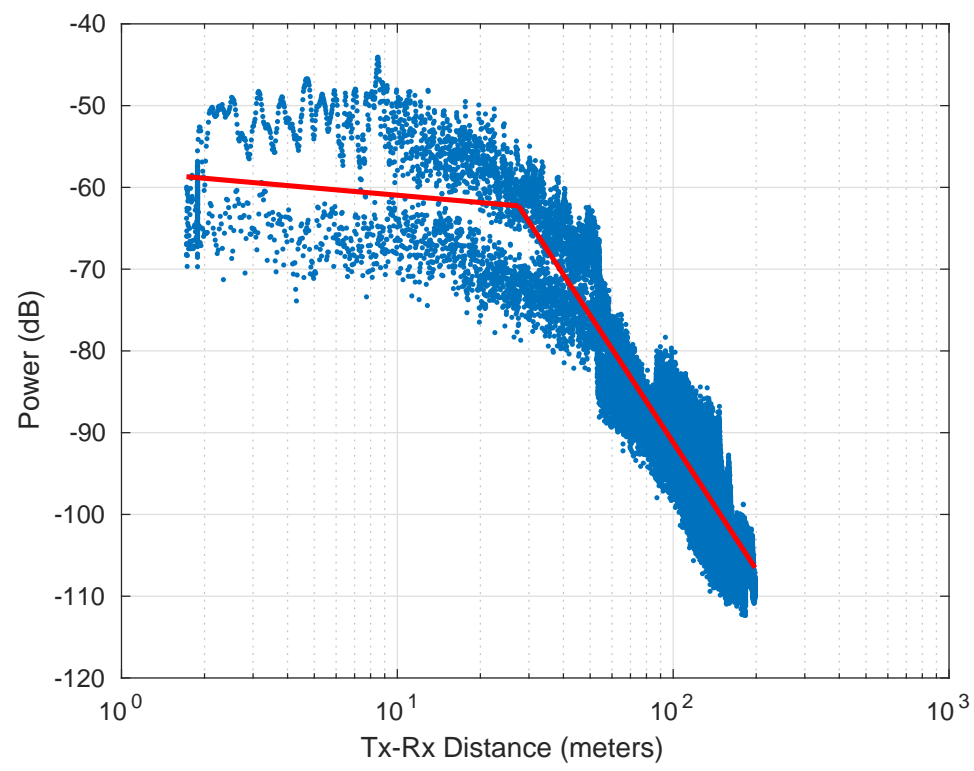

Figure 162: Overall Received Power vs. Distance: Horizontal Polarization, 2.245GHz, T1, Outer Path.

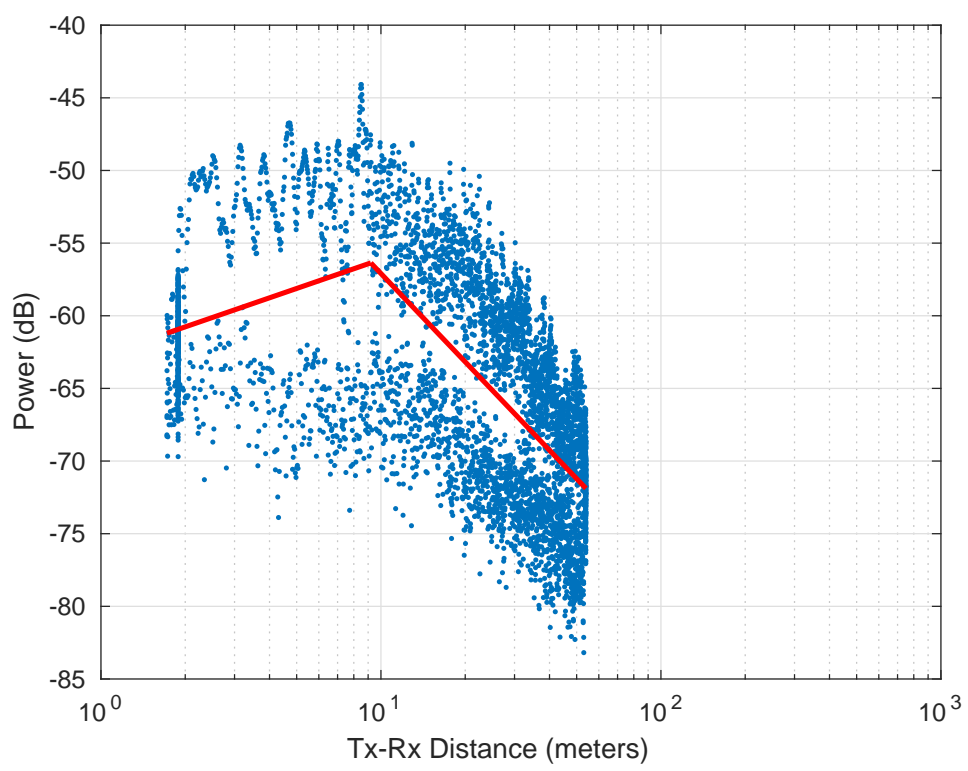

Figure 163: Line of Sight Received Power vs. Distance: Horizontal Polarization, $2.245 \mathrm{GHz}, \mathrm{T} 1$, Outer Path. 


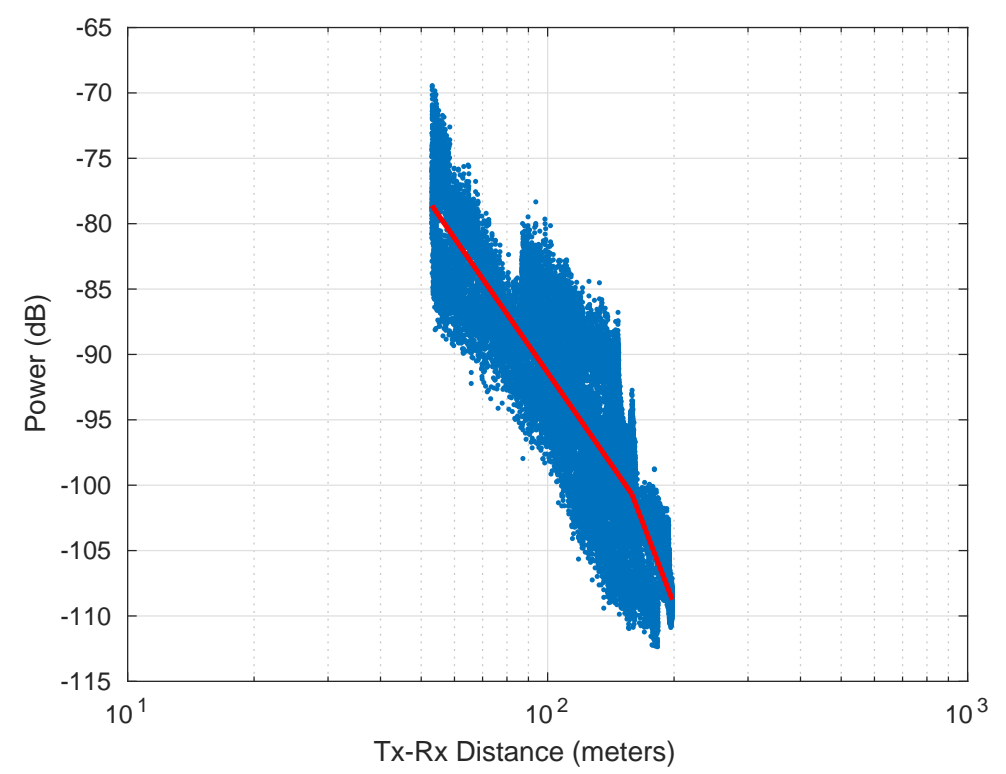

Figure 164: Non Line of Sight Received Power vs. Distance: Horizontal Polarization, $2.245 \mathrm{GHz}, \mathrm{T} 1$, Outer Path.

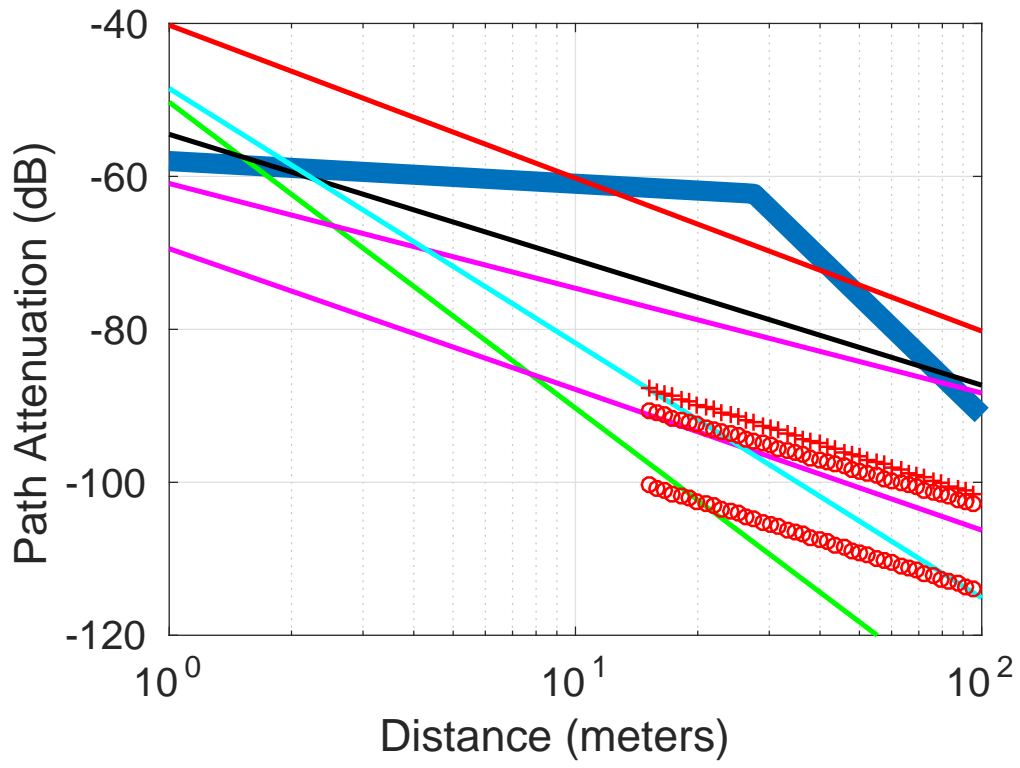

Figure 165: Comparison to other models: Horizontal Polarization, 2.245GHz, T1, Outer Path. 


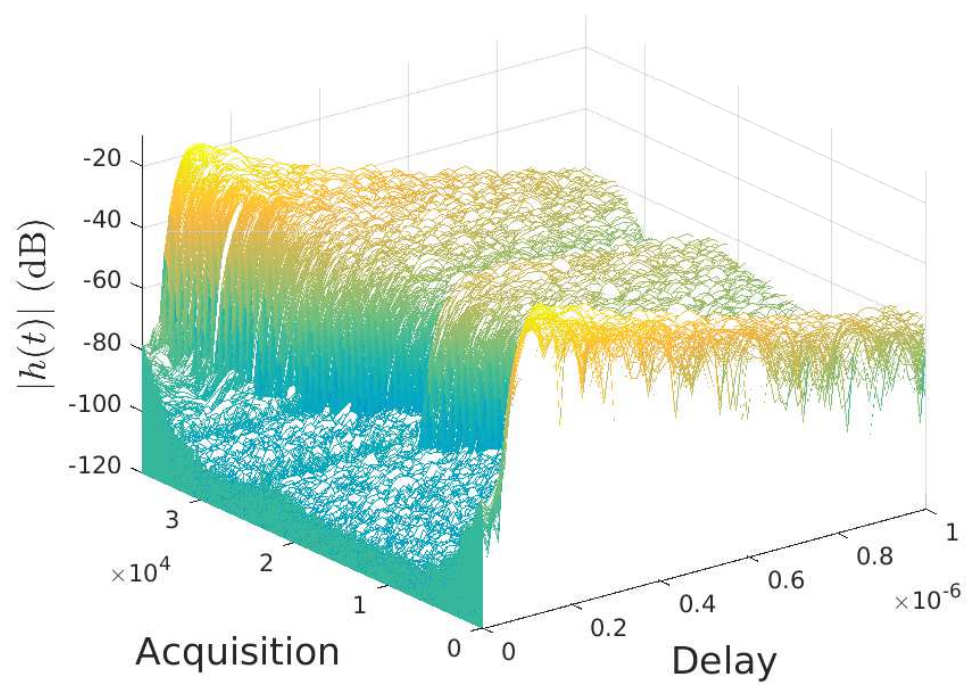

Figure 166: Impulse Responses: Horizontal Polarization, 2.245GHz, T1, Outer Path.

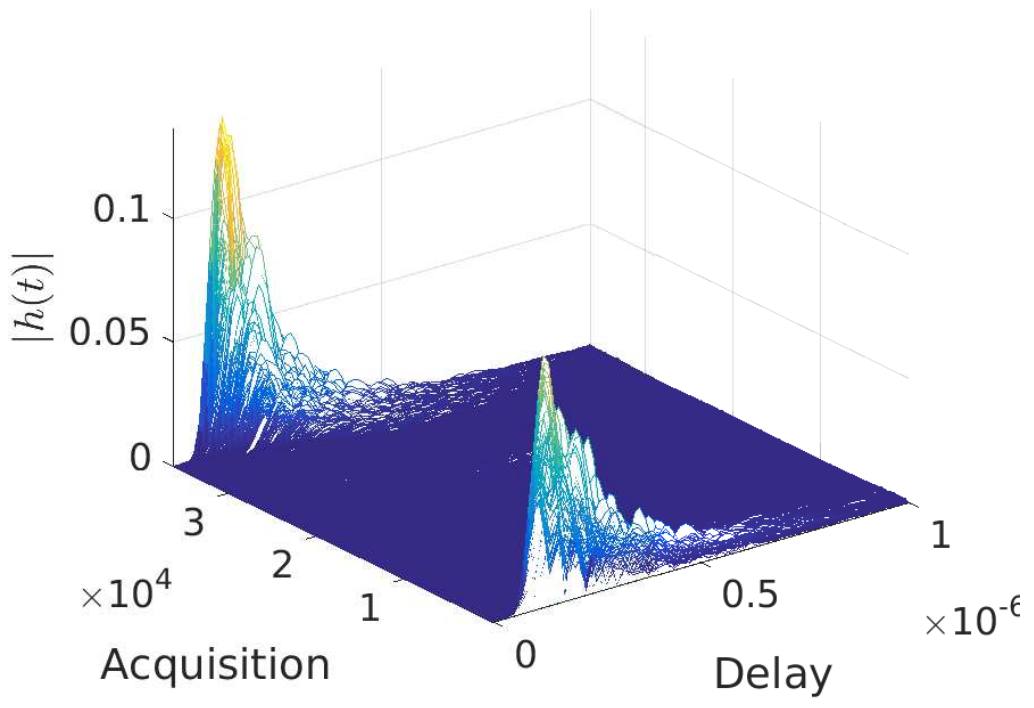

Figure 167: Impulse Responses: Horizontal Polarization, 2.245GHz, T1, Outer Path. 


\section{B.2 Horizontal Polarization, 5.4GHz, Transmitter 1, Outer Path}

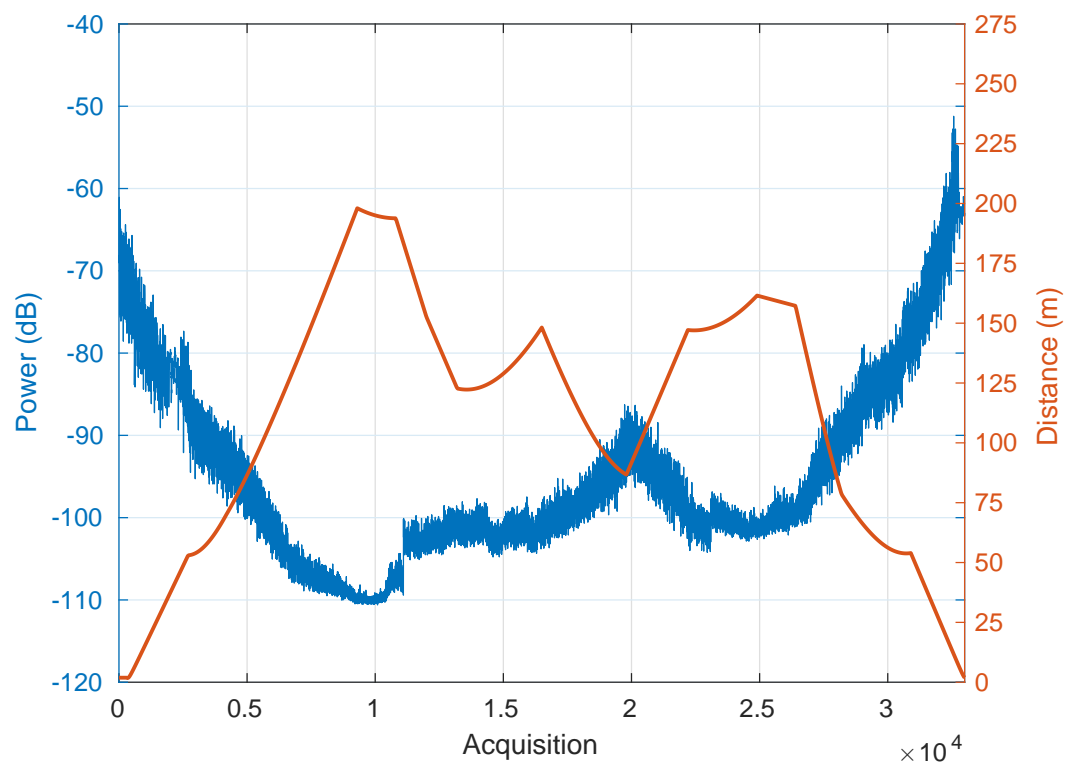

Figure 168: Overall Received Power vs. Acquisition Number and Distance vs. Acquisition Number: Horizontal Polarization, 5.4GHz, T1, Outer Path.

\begin{tabular}{c|c|c|c|c|c}
\hline$\alpha_{1}$ & $\alpha_{2}$ & $\beta(\mathrm{m})$ & $k_{1}(\mathrm{~dB})$ & $k_{2}(\mathrm{~dB})$ & $\mathrm{MSE}$ \\
\hline 0.48352 & 4.1607 & 29.7219 & -66.2907 & -12.1234 & 11.7267
\end{tabular}

Table 31: Parameters for Horizontal Polarization, 5.4GHz, T1, Outer Path 


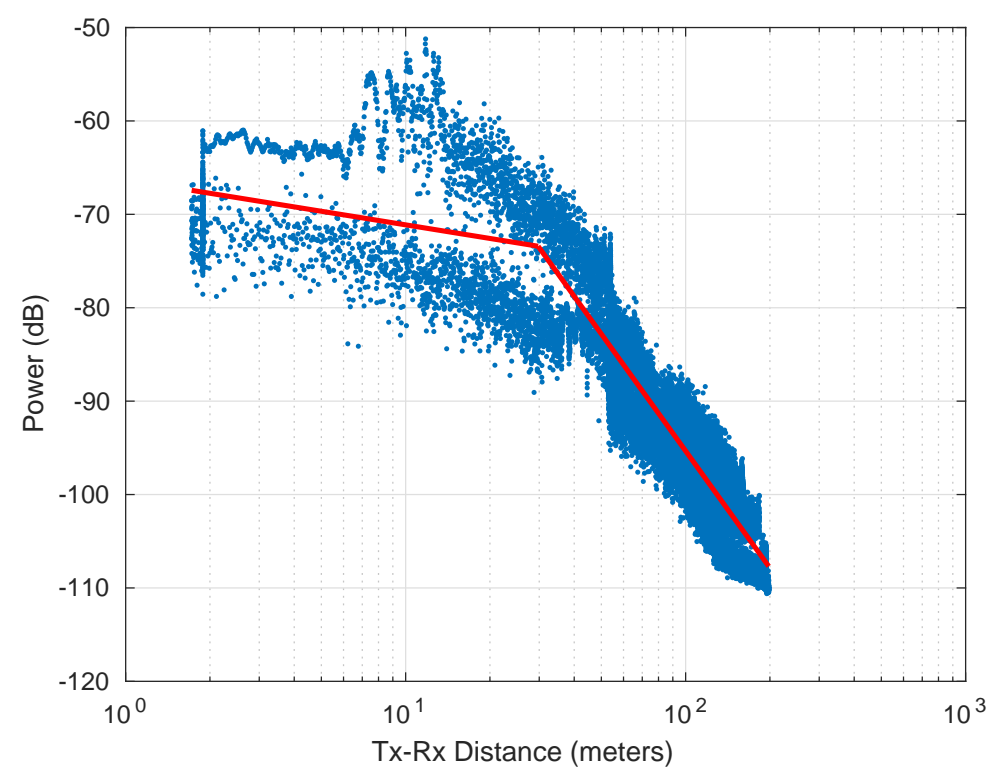

Figure 169: Overall Received Power vs. Distance: Horizontal Polarization, 5.4GHz, T1, Outer Path.

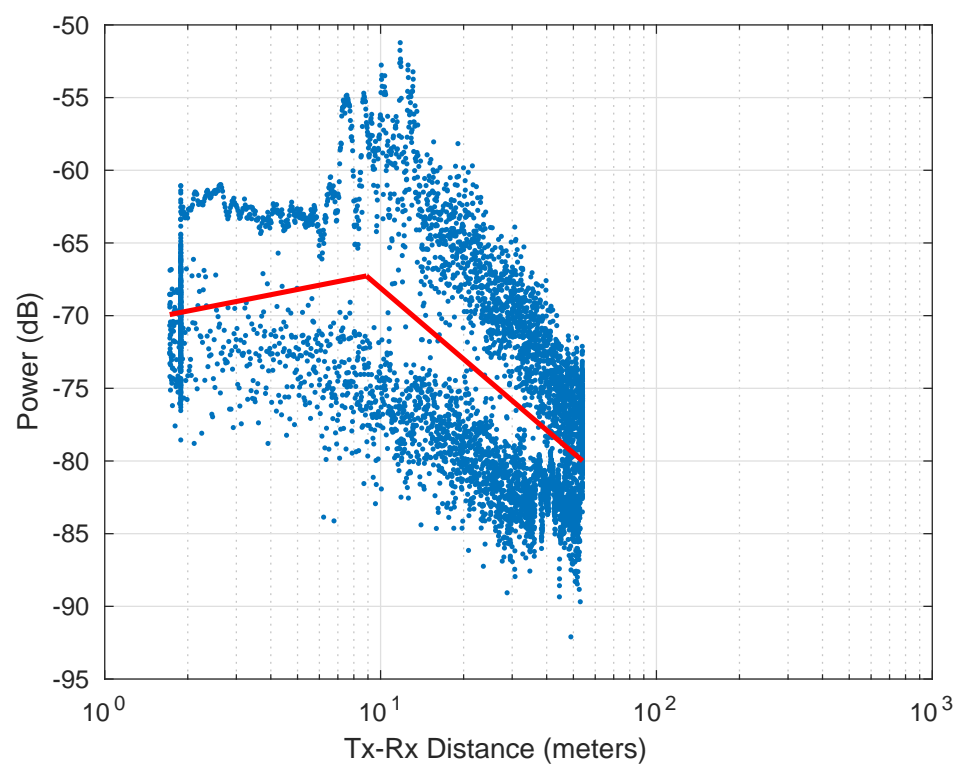

Figure 170: Line of Sight Received Power vs. Distance: Horizontal Polarization, 5.4GHz, T1, Outer Path. 


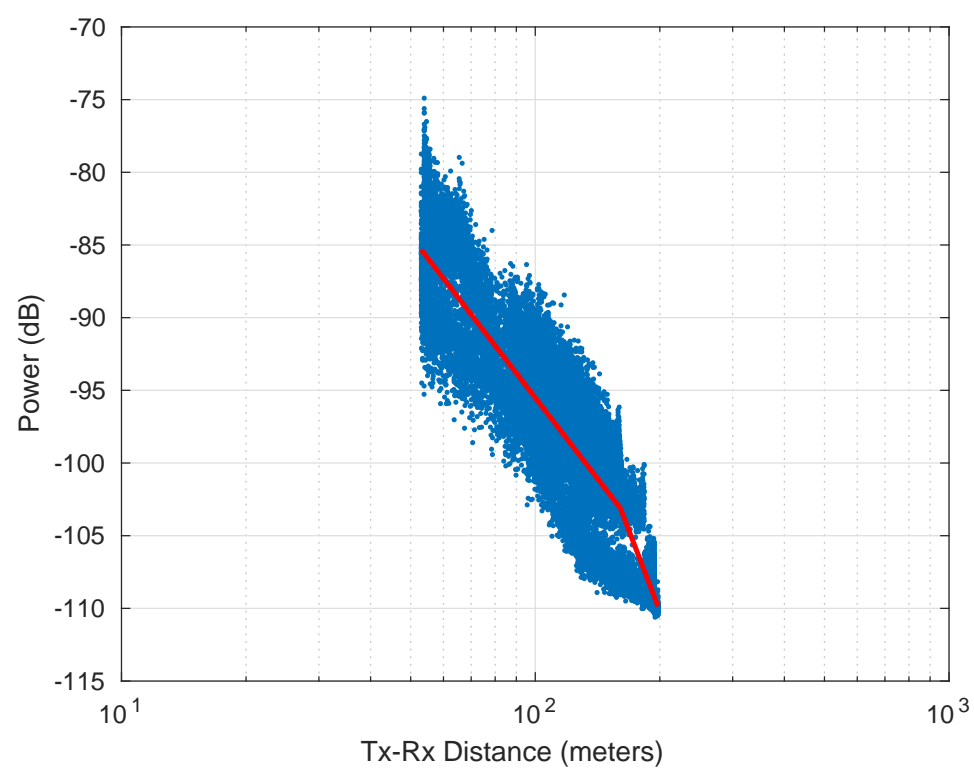

Figure 171: Non Line of Sight Received Power vs. Distance: Horizontal Polarization, $5.4 \mathrm{GHz}, \mathrm{T} 1$, Outer Path.

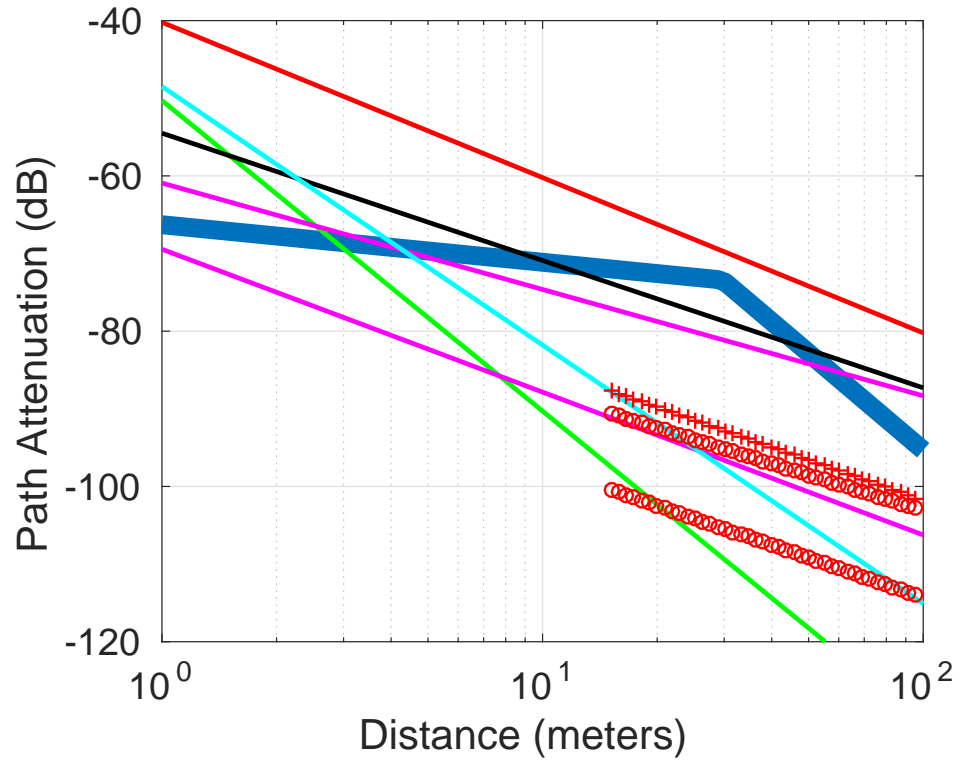

Figure 172: Comparison to other models: Horizontal Polarization, 5.4GHz, T1, Outer Path. 


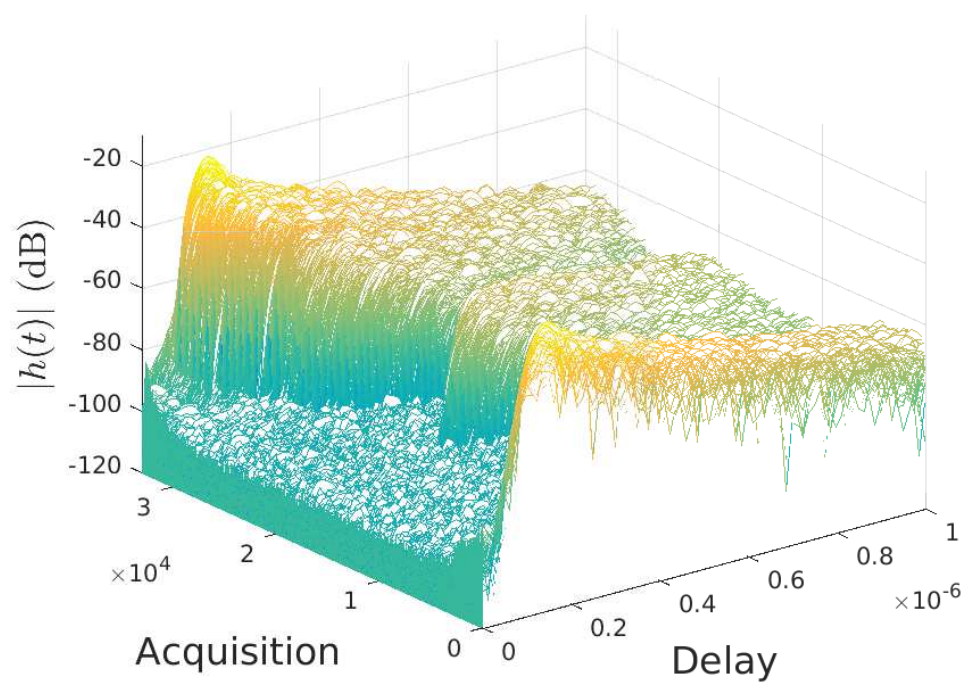

Figure 173: Impulse Responses: Horizontal Polarization, 5.4GHz, T1, Outer Path.

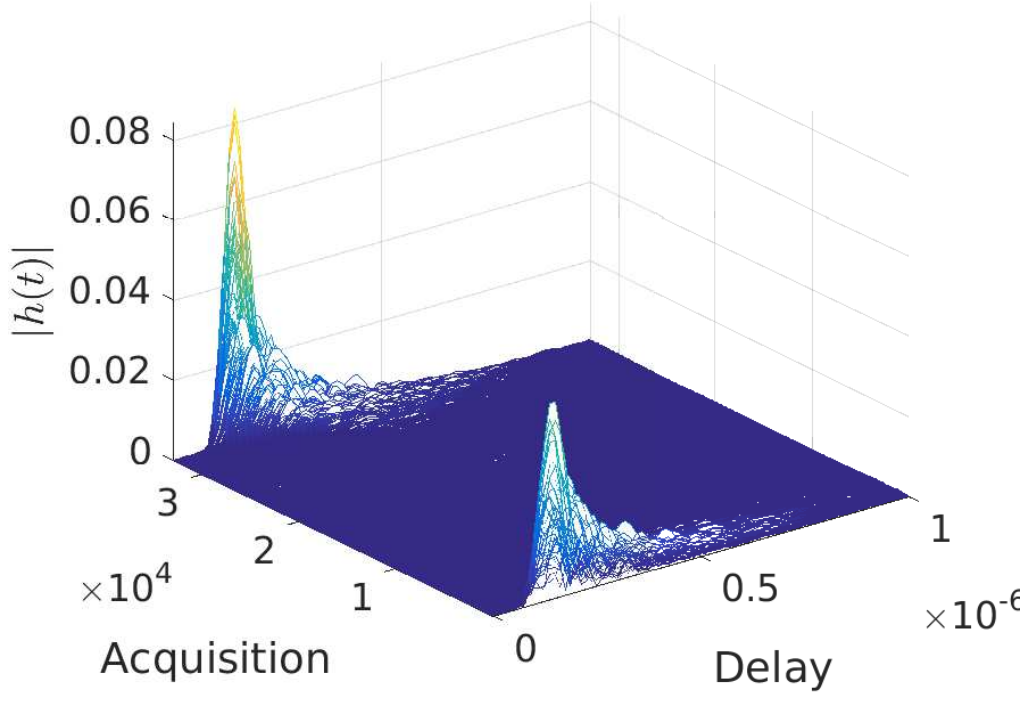

Figure 174: Impulse Responses: Horizontal Polarization, 5.4GHz, T1, Outer Path. 


\section{B.3 Horizontal Polarization, 2.245GHz, Transmitter 1, Inner Path}

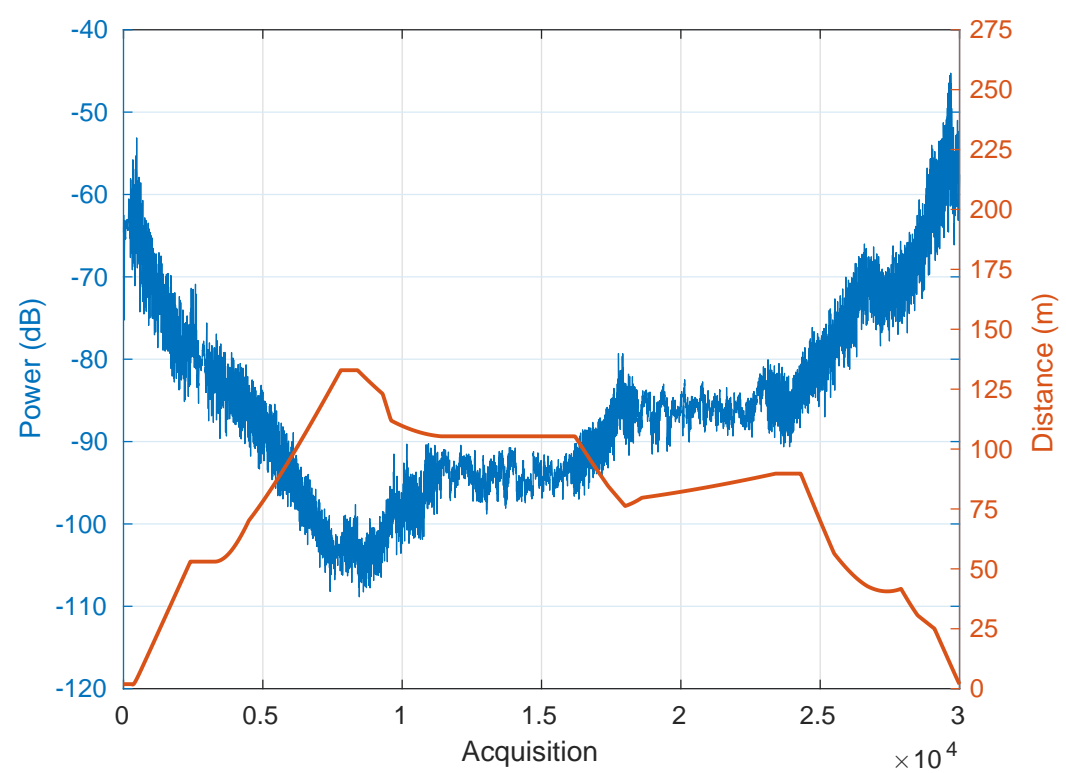

Figure 175: Overall Received Power vs. Acquisition Number and Distance vs. Acquisition Number: Horizontal Polarization, 2.245GHz, T1, Inner Path.

\begin{tabular}{c|c|c|c|c|c}
\hline$\alpha_{1}$ & $\alpha_{2}$ & $\beta(\mathrm{m})$ & $k_{1}(\mathrm{~dB})$ & $k_{2}(\mathrm{~dB})$ & MSE \\
\hline 0.5027 & 5.5783 & 32.7876 & -58.3326 & 18.5988 & 11.4318
\end{tabular}

Table 32: Parameters for Horizontal Polarization, 2.245GHz, T1, Inner Path 


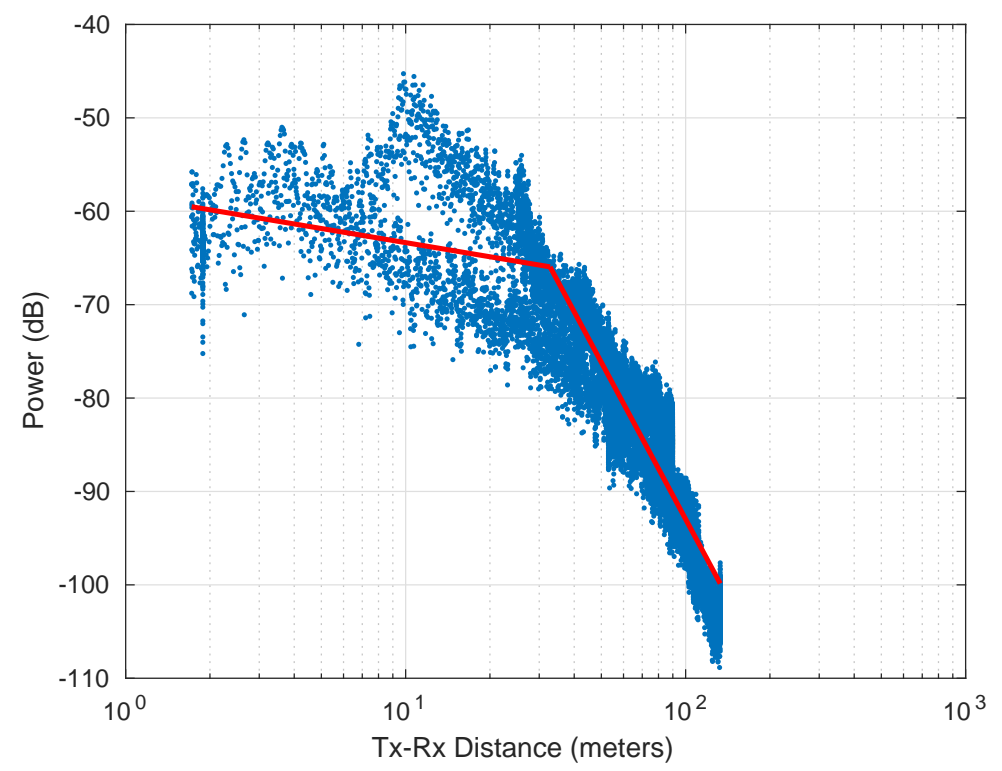

Figure 176: Overall Received Power vs. Distance: Horizontal Polarization, 2.245GHz, T1, Inner Path.

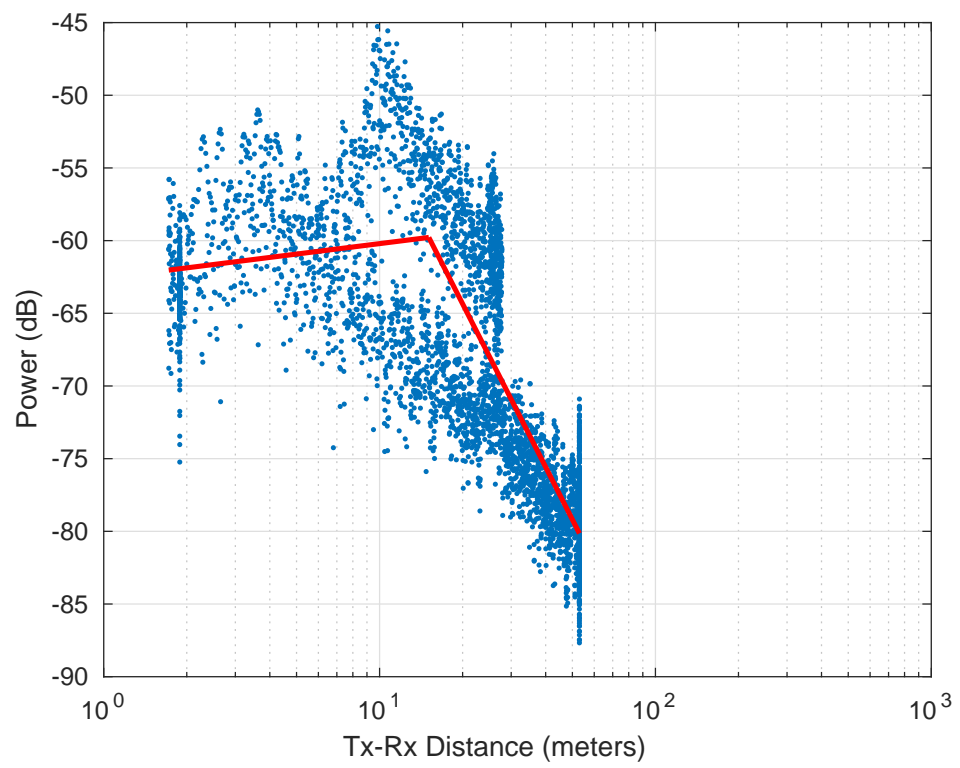

Figure 177: Line of Sight Received Power vs. Distance: Horizontal Polarization, $2.245 \mathrm{GHz}, \mathrm{T} 1$, Inner Path. 


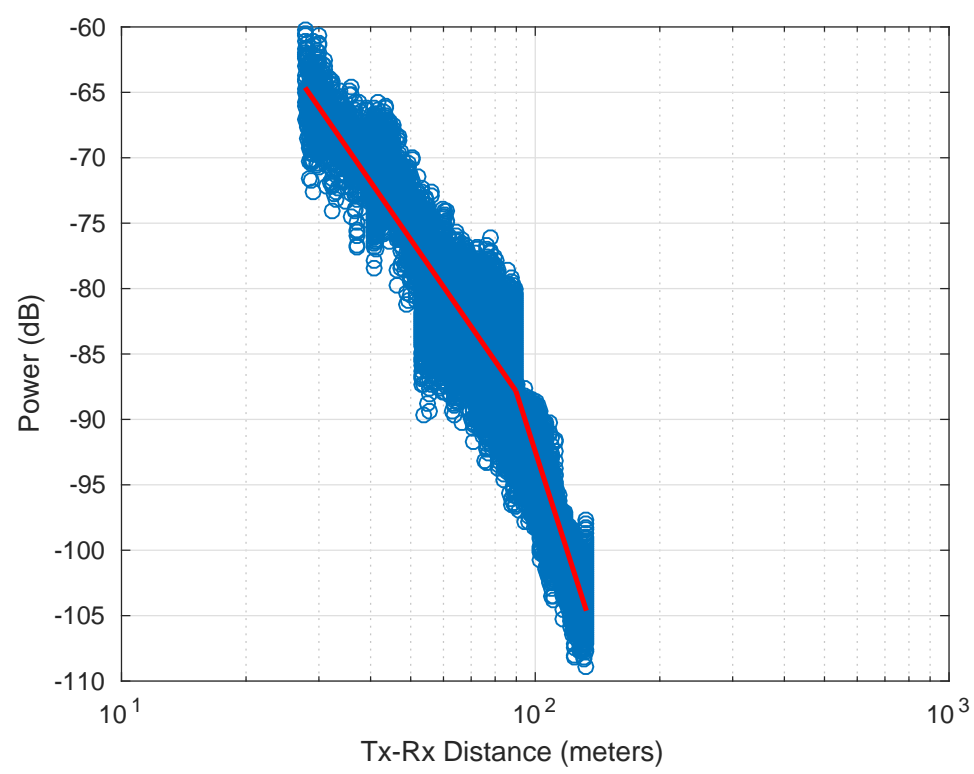

Figure 178: Non Line of Sight Received Power vs. Distance: Horizontal Polarization, $2.245 \mathrm{GHz}, \mathrm{T} 1$, Inner Path.

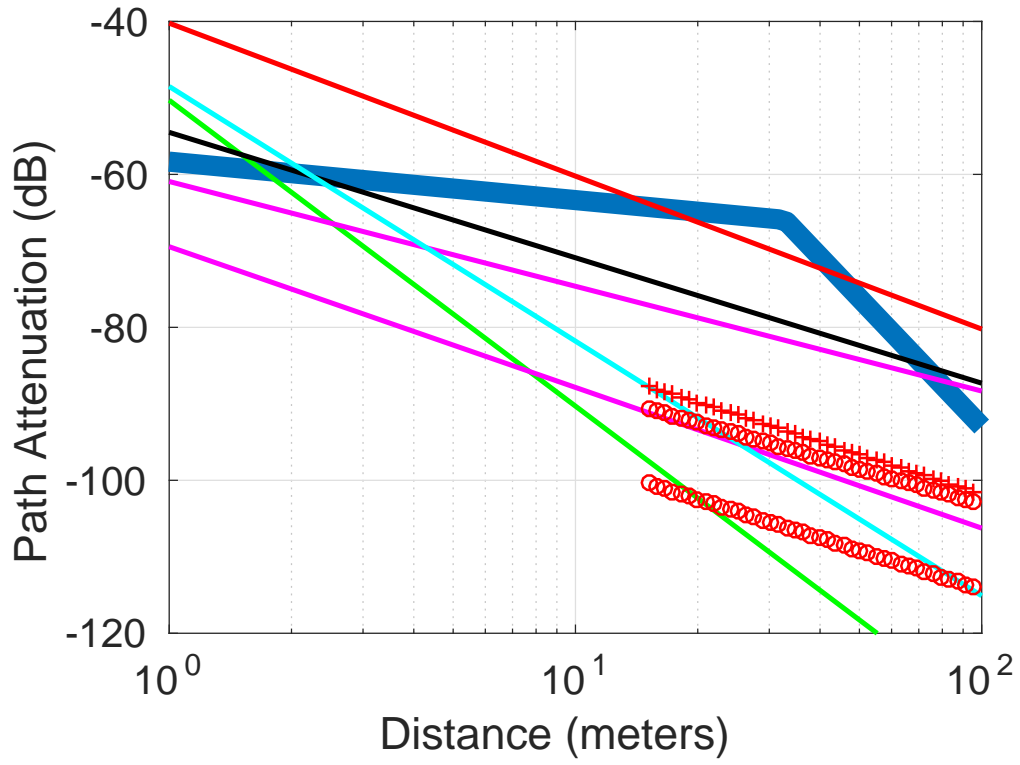

Figure 179: Comparison to other models: Horizontal Polarization, 2.245GHz, T1, Inner Path. 


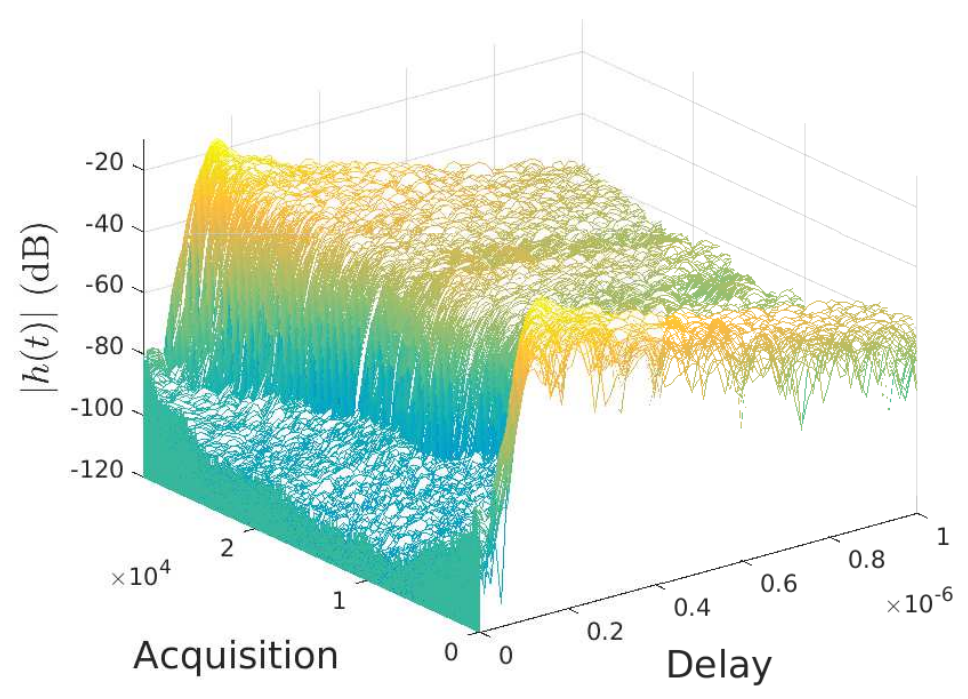

Figure 180: Impulse Responses: Horizontal Polarization, 2.245GHz, T1, Inner Path.

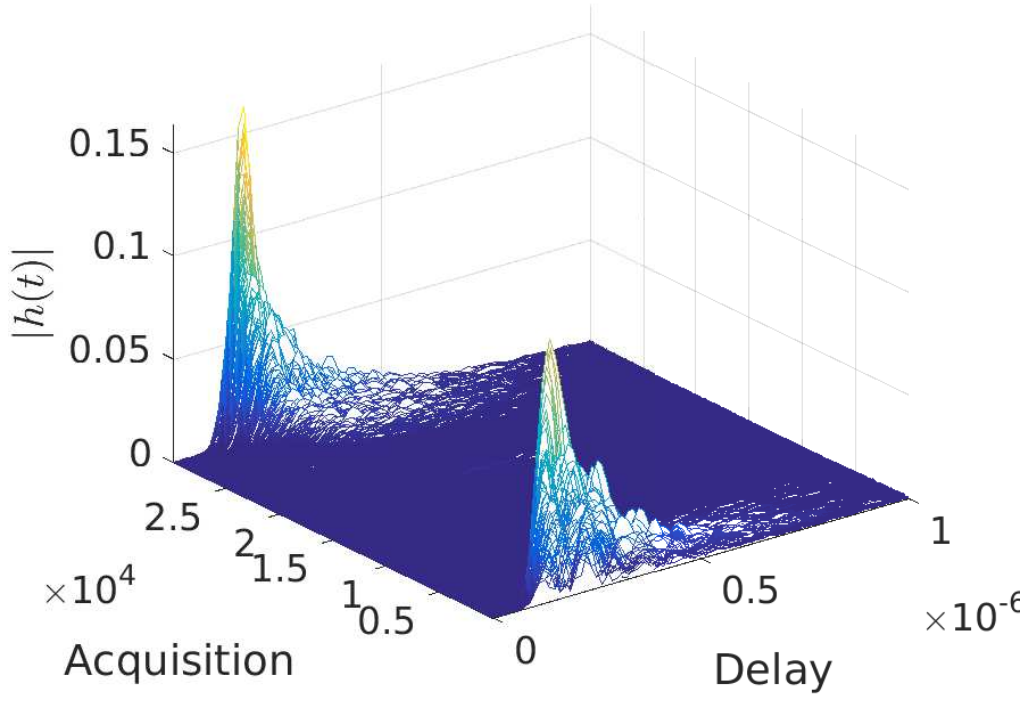

Figure 181: Impulse Responses: Horizontal Polarization, 2.245GHz, T1, Inner Path. 


\section{B.4 Horizontal Polarization, 5.4GHz, Transmitter 1, Inner Path}

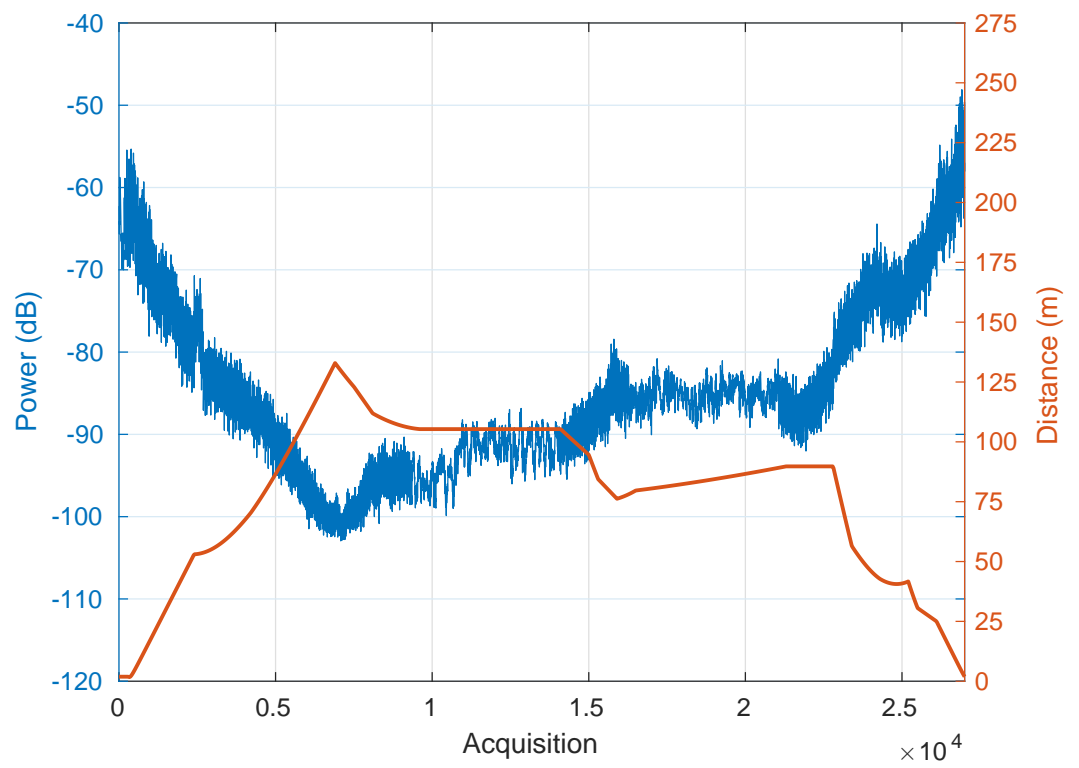

Figure 182: Overall Received Power vs. Acquisition Number and Distance vs. Acquisition Number: Horizontal Polarization, 5.4GHz, T1, Inner Path.

\begin{tabular}{c|c|c|c|c|c}
\hline$\alpha_{1}$ & $\alpha_{2}$ & $\beta(\mathrm{m})$ & $k_{1}(\mathrm{~dB})$ & $k_{2}(\mathrm{~dB})$ & $\mathrm{MSE}$ \\
\hline 0.732 & 5.0265 & 36.55 & -57.8965 & 9.2216 & 10.6267
\end{tabular}

Table 33: Parameters for Horizontal Polarization, 5.4GHz, T1, Inner Path 


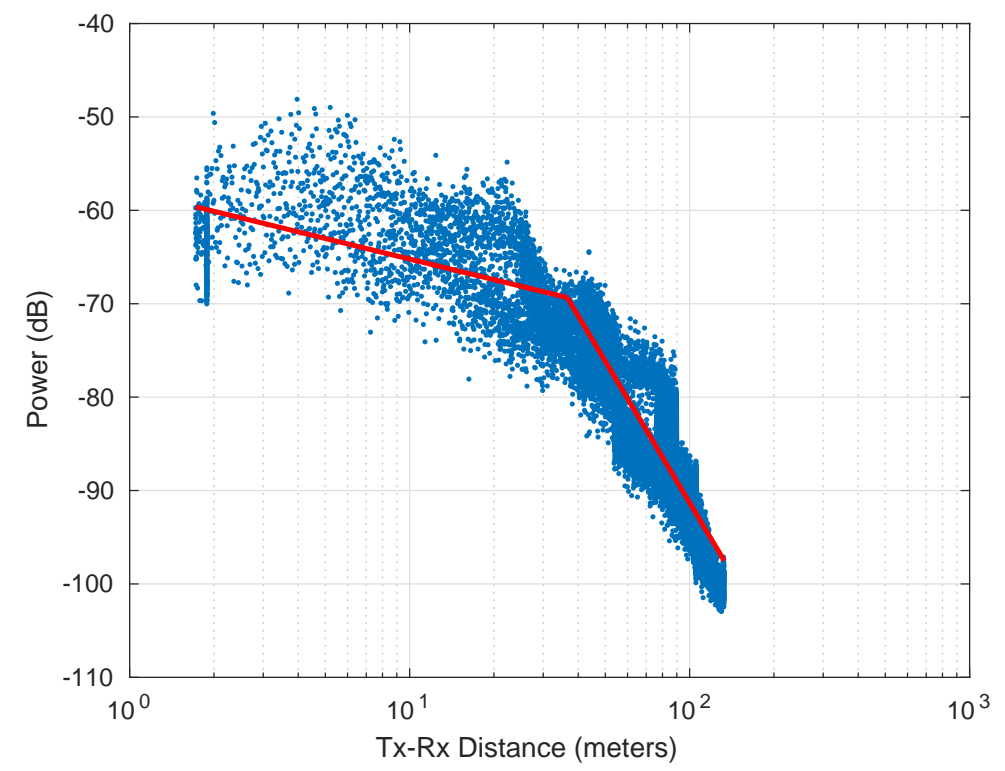

Figure 183: Overall Received Power vs. Distance: Horizontal Polarization, 5.4GHz, T1, Inner Path.

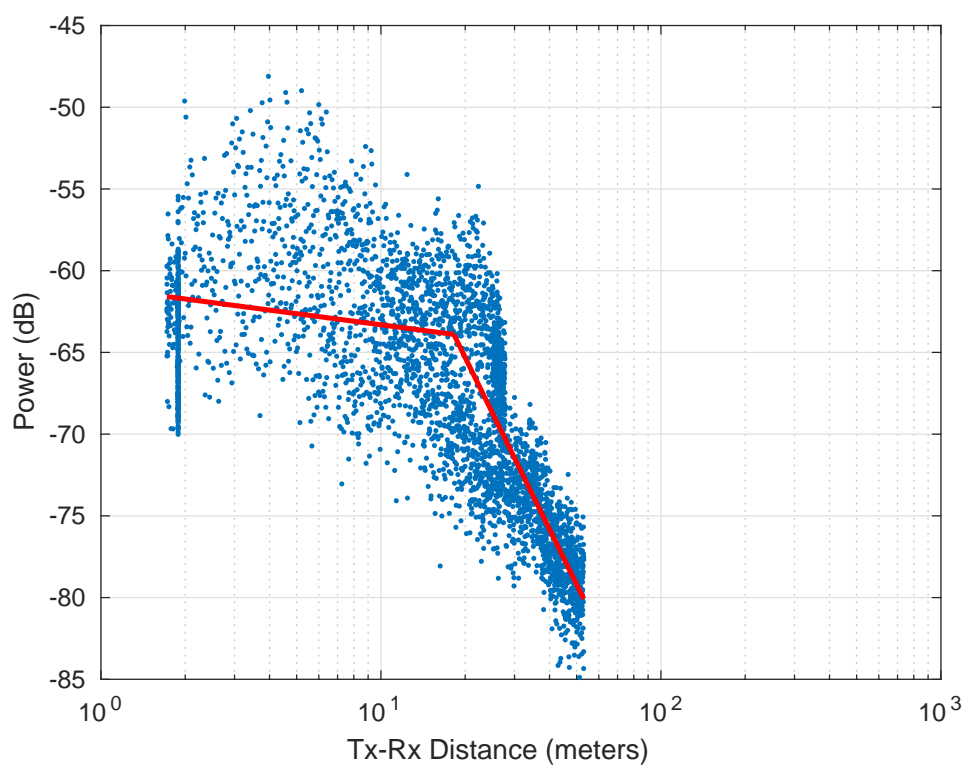

Figure 184: Line of Sight Received Power vs. Distance: Horizontal Polarization, 5.4GHz, T1, Inner Path. 


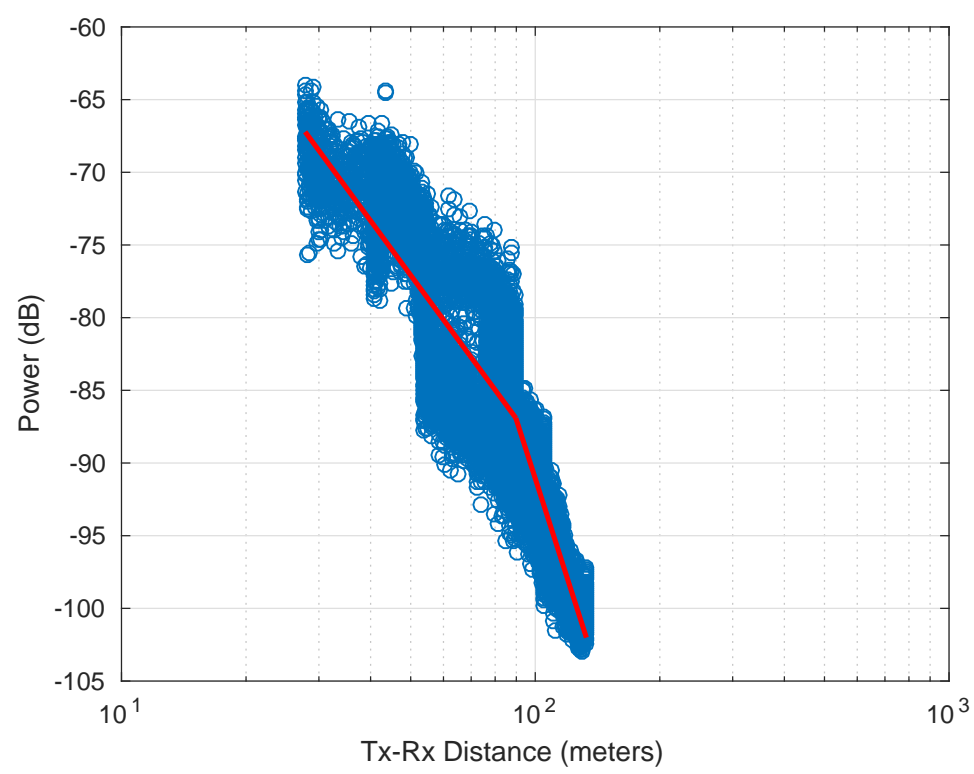

Figure 185: Non Line of Sight Received Power vs. Distance: Horizontal Polarization, $5.4 \mathrm{GHz}, \mathrm{T} 1$, Inner Path.

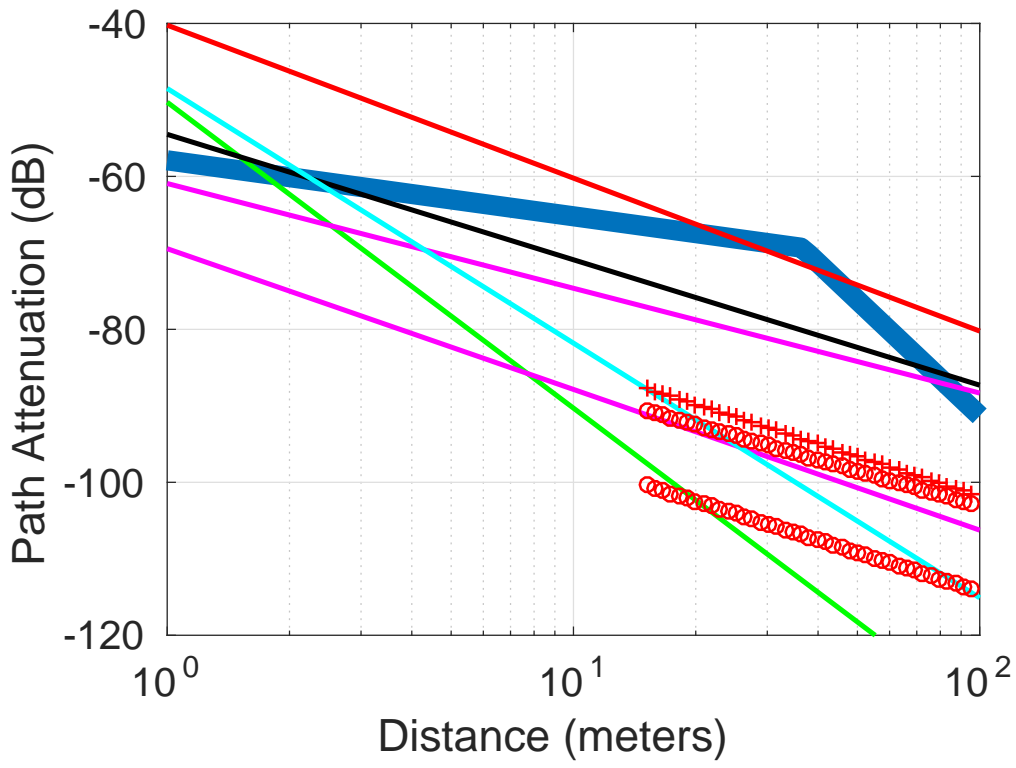

Figure 186: Comparison to other models: Horizontal Polarization, 5.4GHz, T1, Inner Path. 


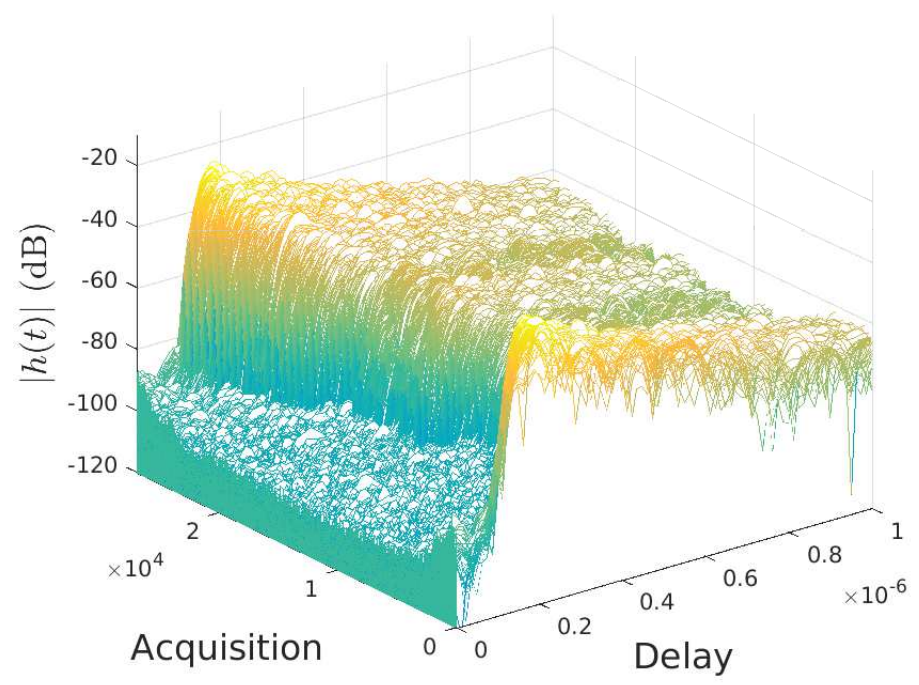

Figure 187: Impulse Responses: Horizontal Polarization, 5.4GHz, T1, Inner Path.

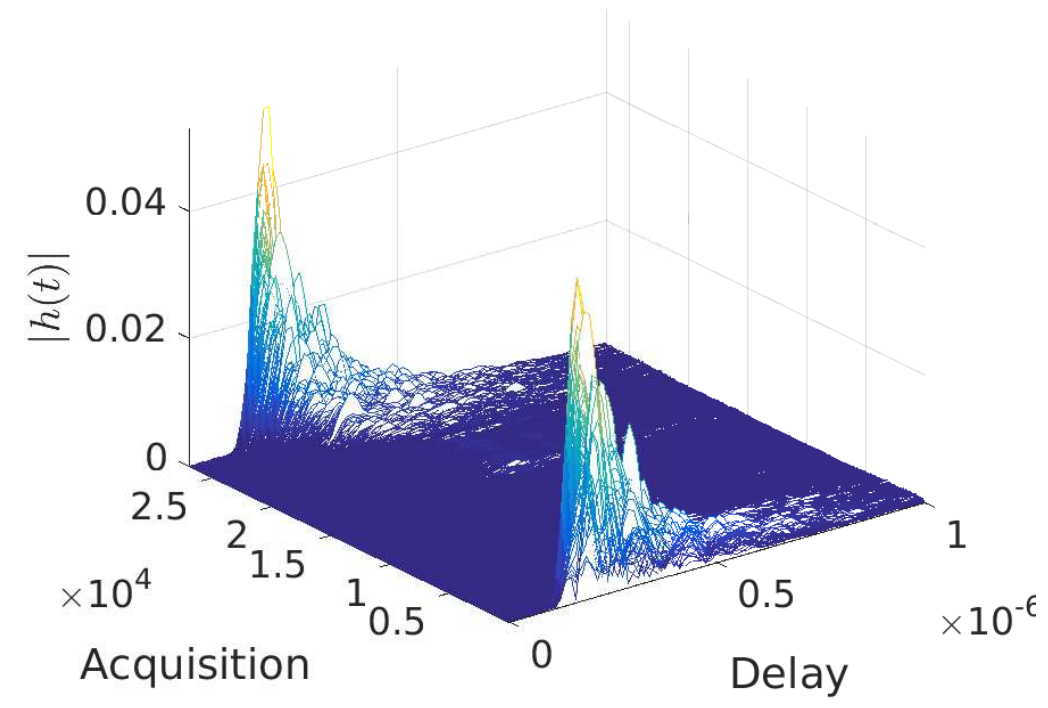

Figure 188: Impulse Responses: Horizontal Polarization, 5.4GHz, T1, Inner Path. 


\section{B.5 Horizontal Polarization, 2.245GHz, Transmitter 2 Outer Path}

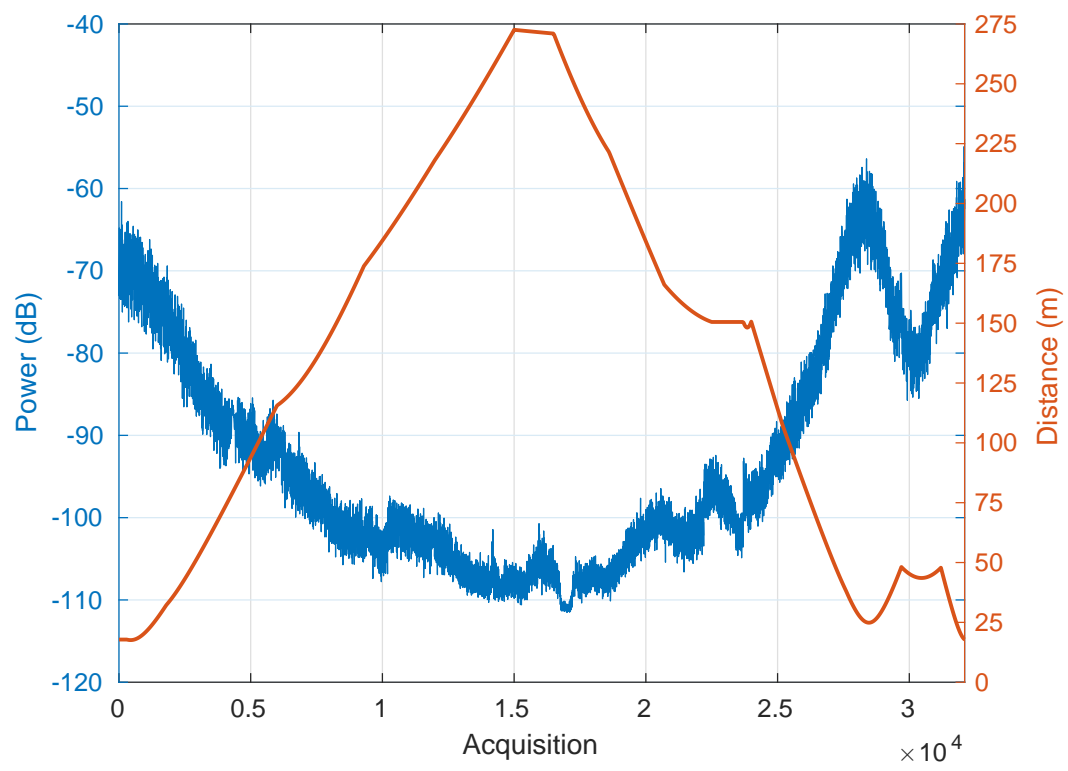

Figure 189: Overall Received Power vs. Acquisition Number and Distance vs. Acquisition Number: Horizontal Polarization, 2.245GHz, T2, Outer Path.

\begin{tabular}{c|c|c|c|c|c}
\hline Frequency & Polarization & $\alpha_{1}$ & $\alpha_{2}$ & $\beta(\mathrm{m})$ & $k_{1}(\mathrm{~dB})$ \\
\hline-1.742 & 4.1473 & 25.3228 & -90.7101 & -8.0524 & 9.6062
\end{tabular}

Table 34: Parameters for Horizontal Polarization, 2.245GHz, T2, Outer Path 


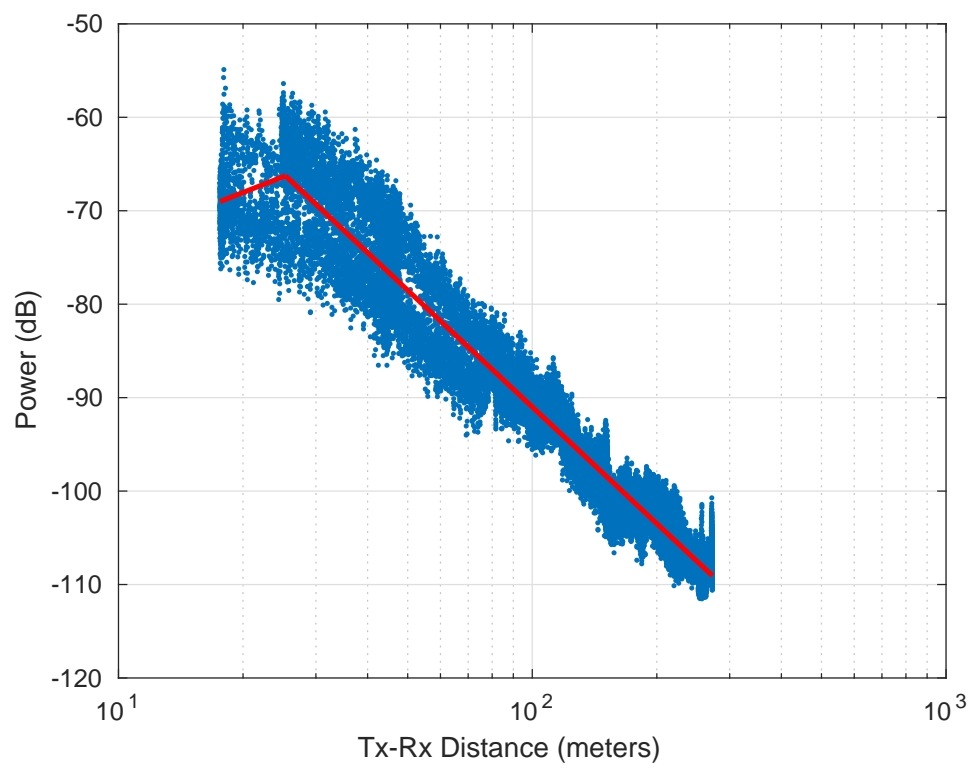

Figure 190: Overall Received Power vs. Distance: Horizontal Polarization, 2.245GHz, T2, Outer Path.

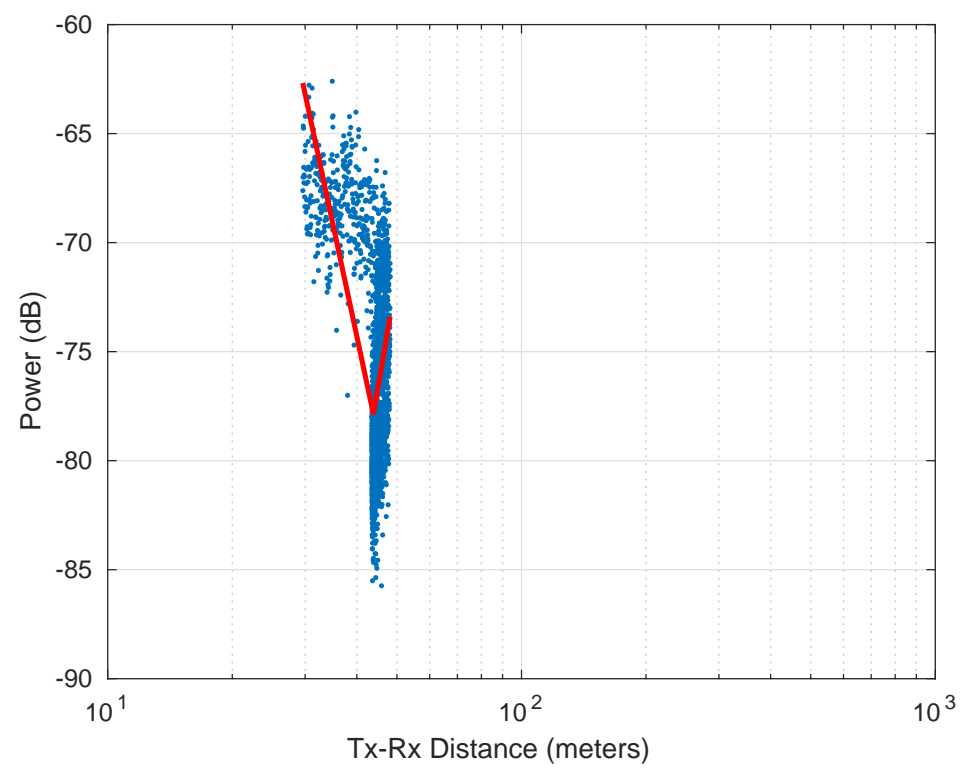

Figure 191: Line of Sight Received Power vs. Distance: Horizontal Polarization, $2.245 \mathrm{GHz}, \mathrm{T} 2$, Outer Path. 


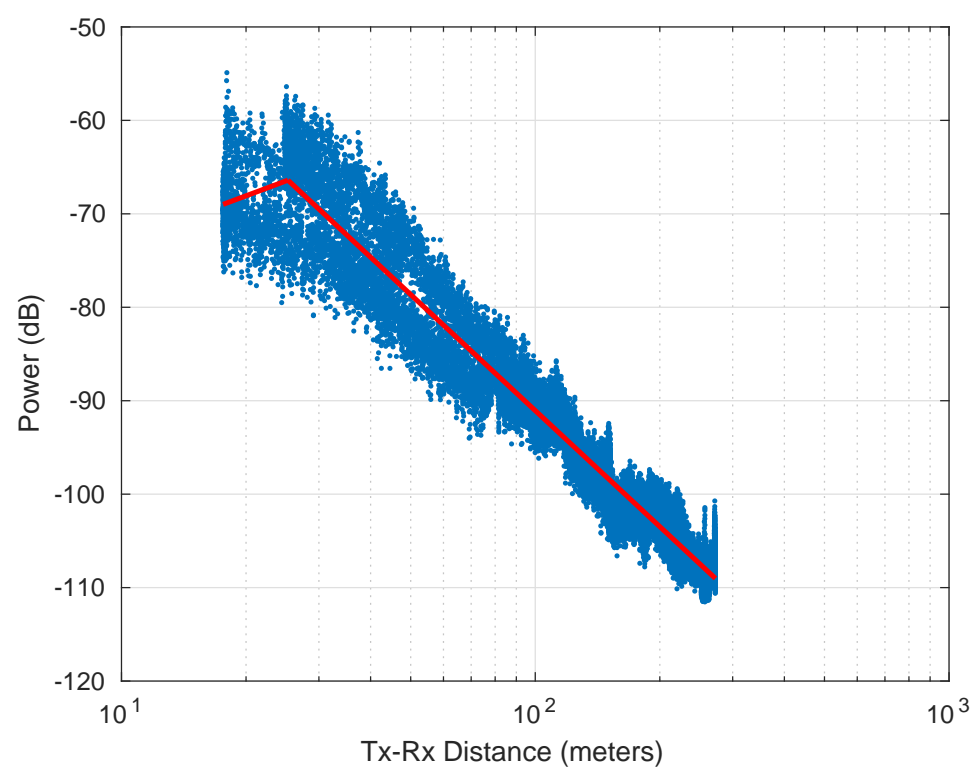

Figure 192: Non Line of Sight Received Power vs. Distance: Horizontal Polarization, $2.245 \mathrm{GHz}, \mathrm{T} 2$, Outer Path.

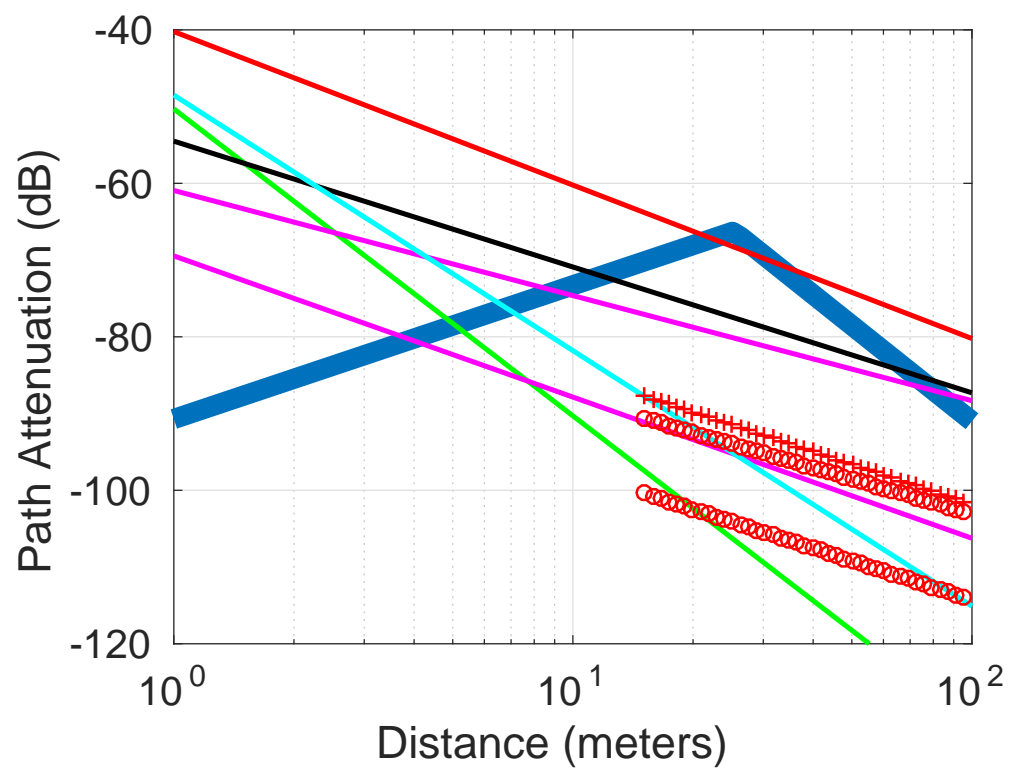

Figure 193: Comparison to other models: Horizontal Polarization, 2.245GHz, T2, Outer Path. 


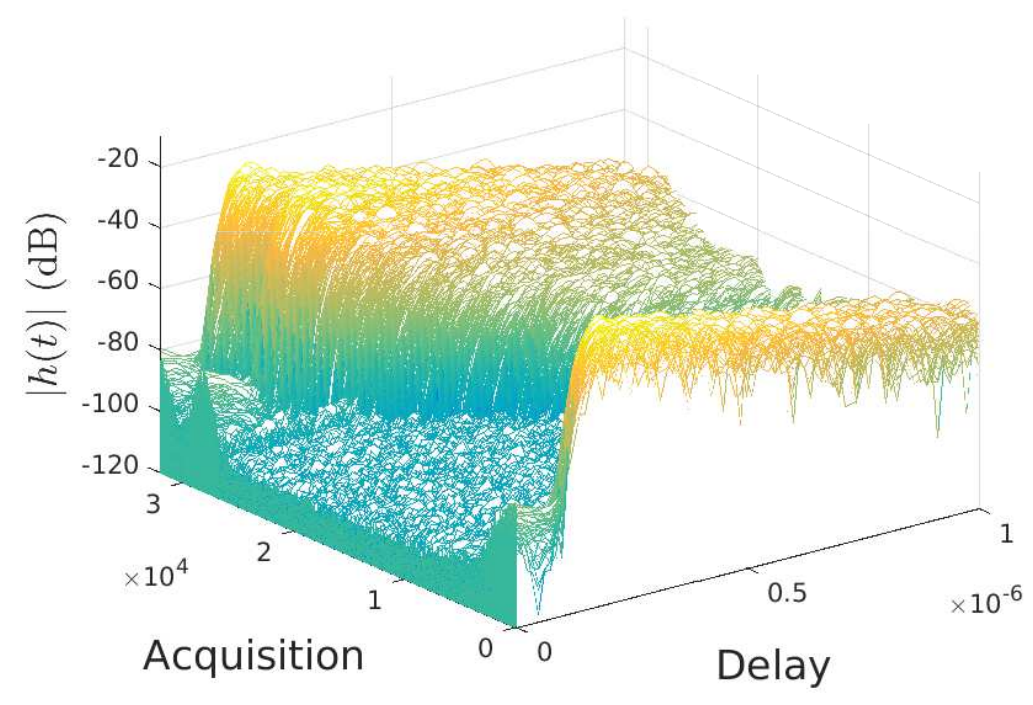

Figure 194: Impulse Responses: Horizontal Polarization, 2.245GHz, T2, Outer Path.

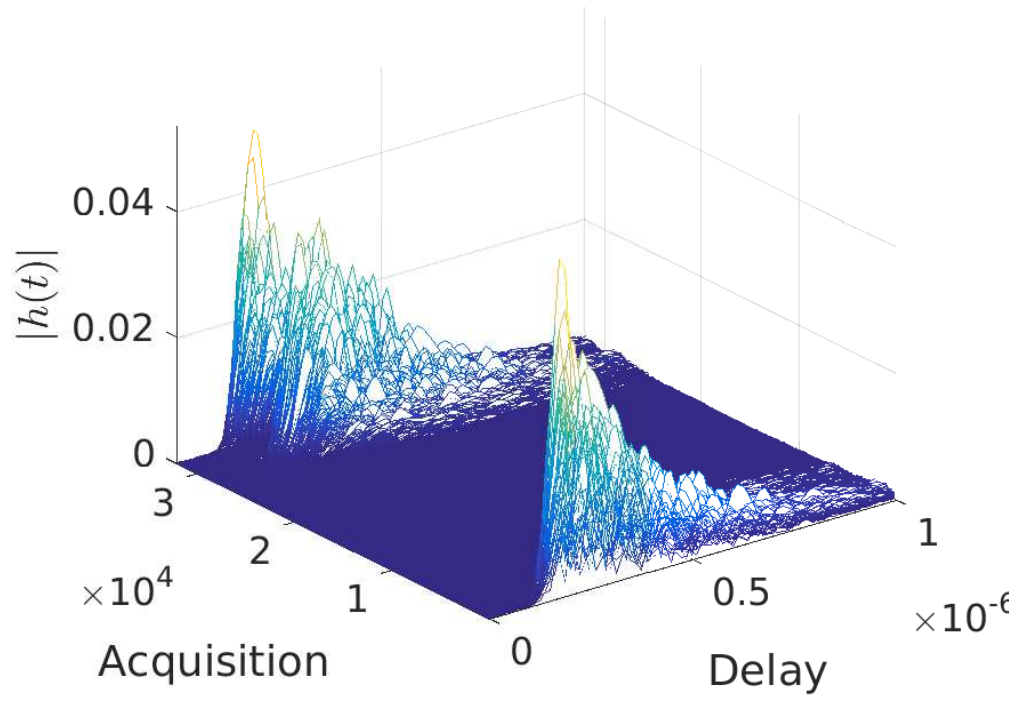

Figure 195: Impulse Responses: Horizontal Polarization, 2.245GHz, T2, Outer Path. 


\section{B.6 Horizontal Polarization, 5.4GHz, Transmitter 2, Outer Path}

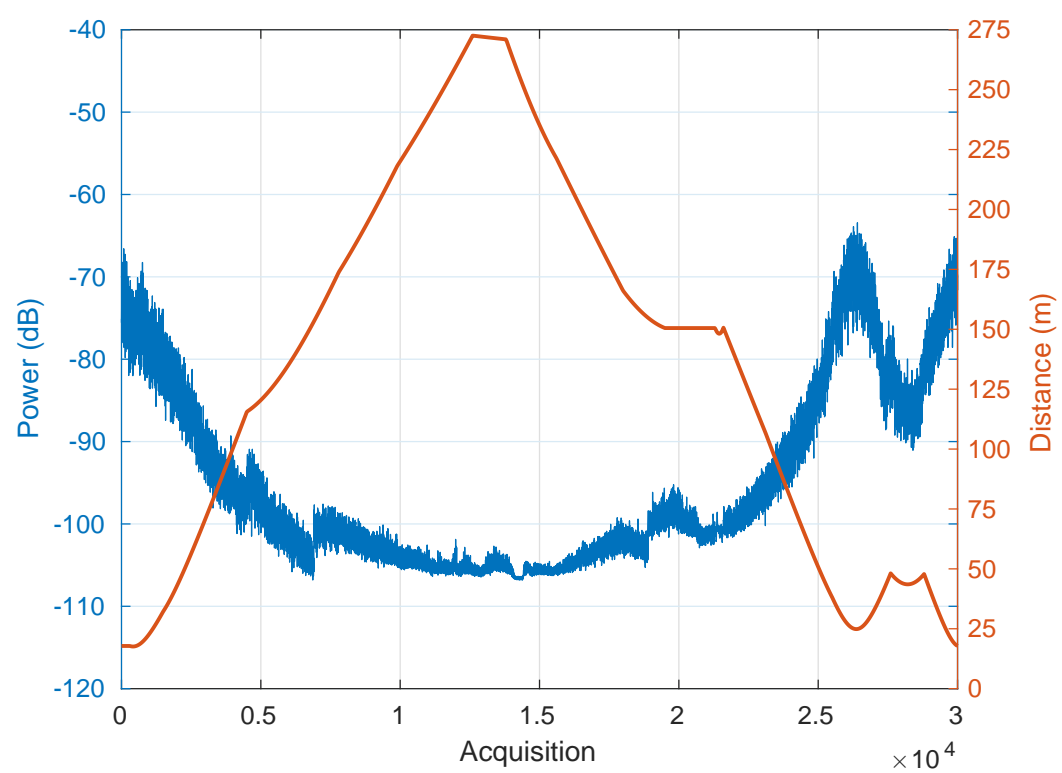

Figure 196: Overall Received Power vs. Acquisition Number and Distance vs. Acquisition Number: Horizontal Polarization, 5.4GHz, T2, Outer Path.

\begin{tabular}{c|c|c|c|c|c}
\hline Frequency & Polarization & $\alpha_{1}$ & $\alpha_{2}$ & $\beta(\mathrm{m})$ & $k_{1}(\mathrm{~dB})$ \\
\hline 3.3884 & 2.0603 & 143.51 & -27.2338 & -55.878 & 7.5173
\end{tabular}

Table 35: Parameters for Horizontal Polarization, 5.4GHz, T2, Outer Path 


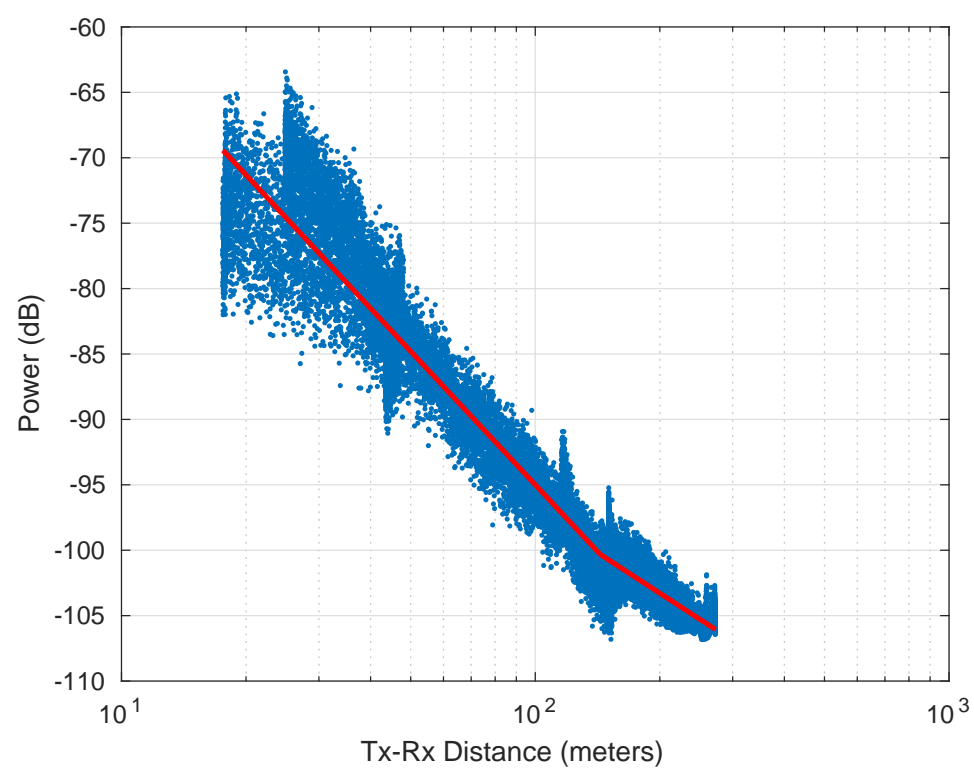

Figure 197: Overall Received Power vs. Distance: Horizontal Polarization, 5.4GHz, T2, Outer Path.

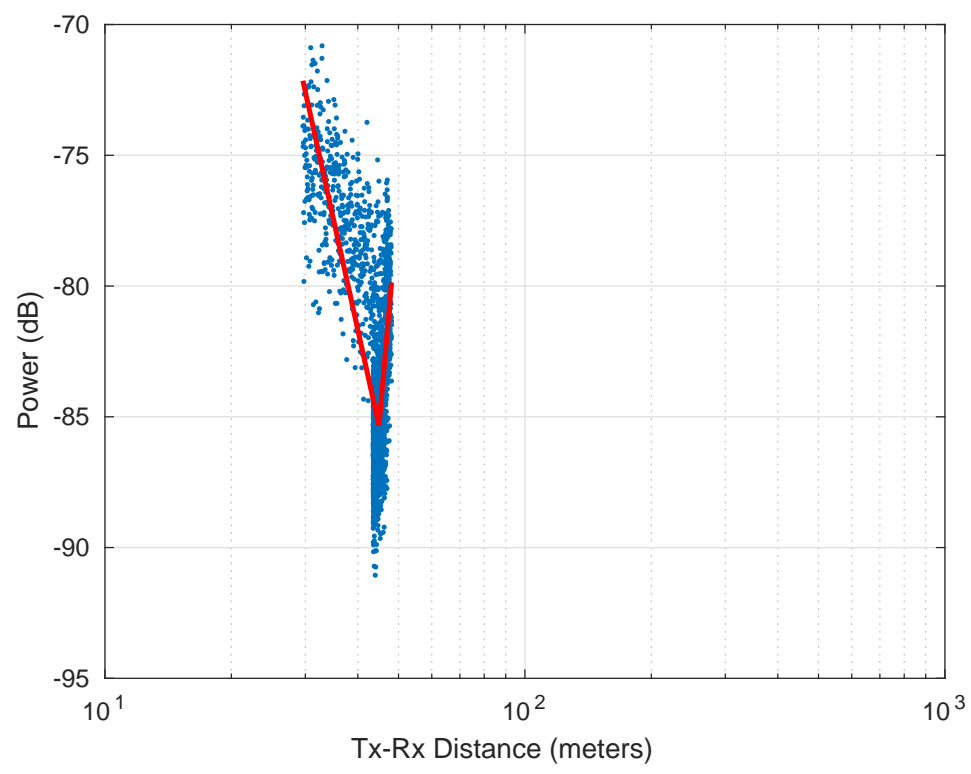

Figure 198: Line of Sight Received Power vs. Distance: Horizontal Polarization, 5.4GHz, T2, Outer Path. 


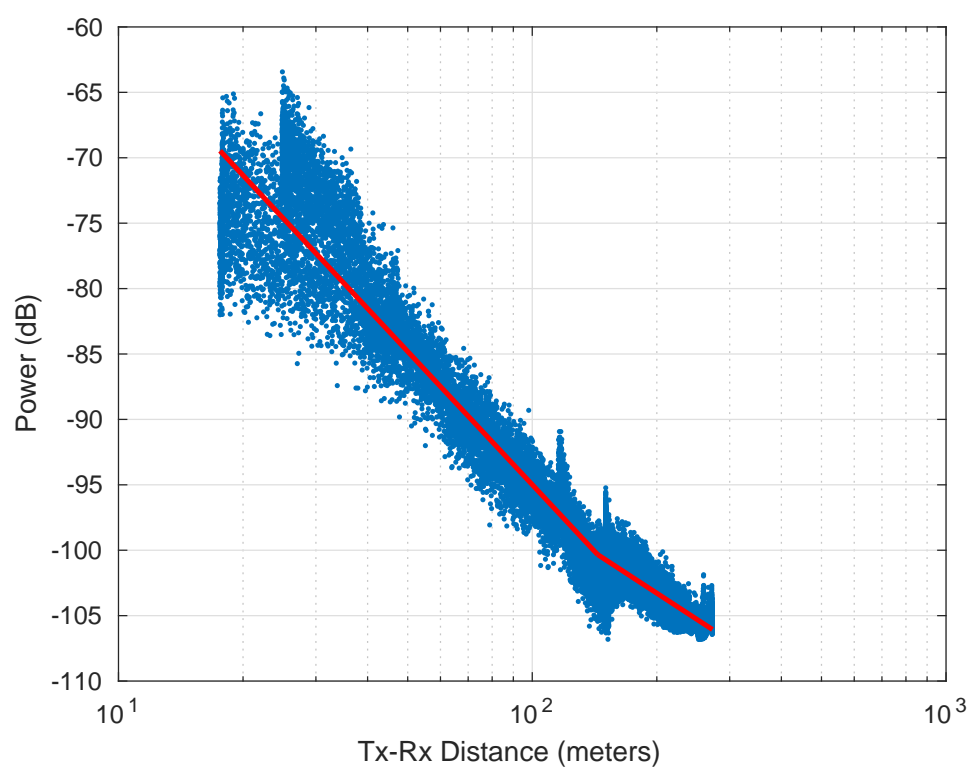

Figure 199: Non Line of Sight Received Power vs. Distance: Horizontal Polarization, $5.4 \mathrm{GHz}, \mathrm{T} 2$, Outer Path.

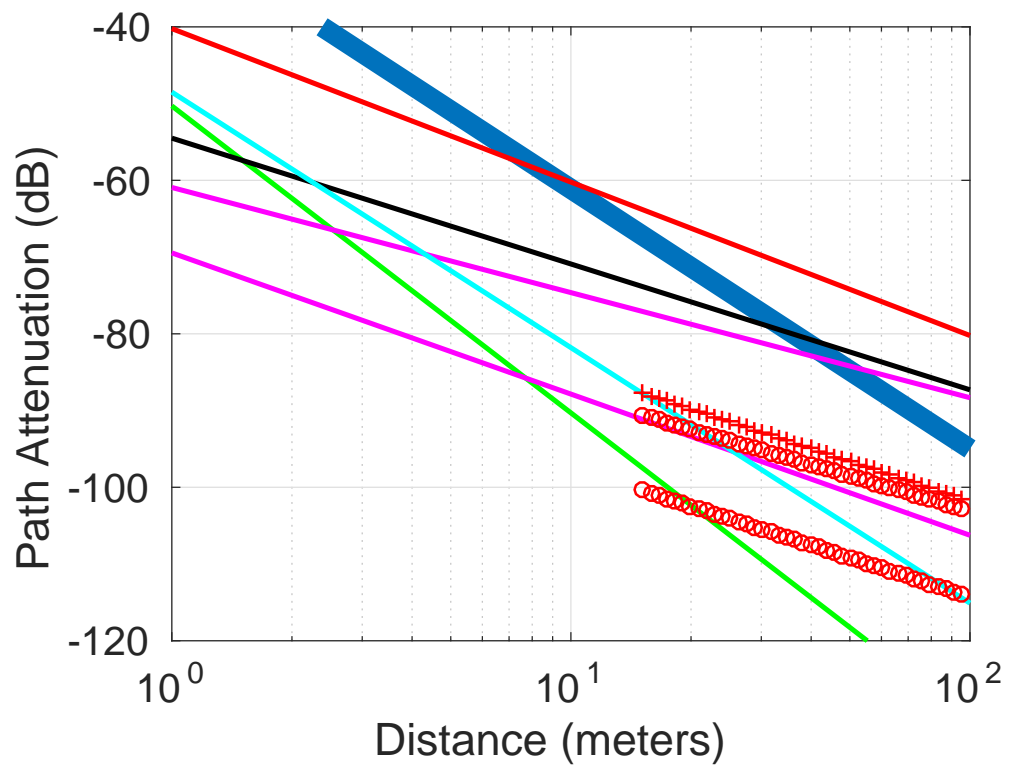

Figure 200: Comparison to other models: Horizontal Polarization, 5.4GHz, T2, Outer Path. 


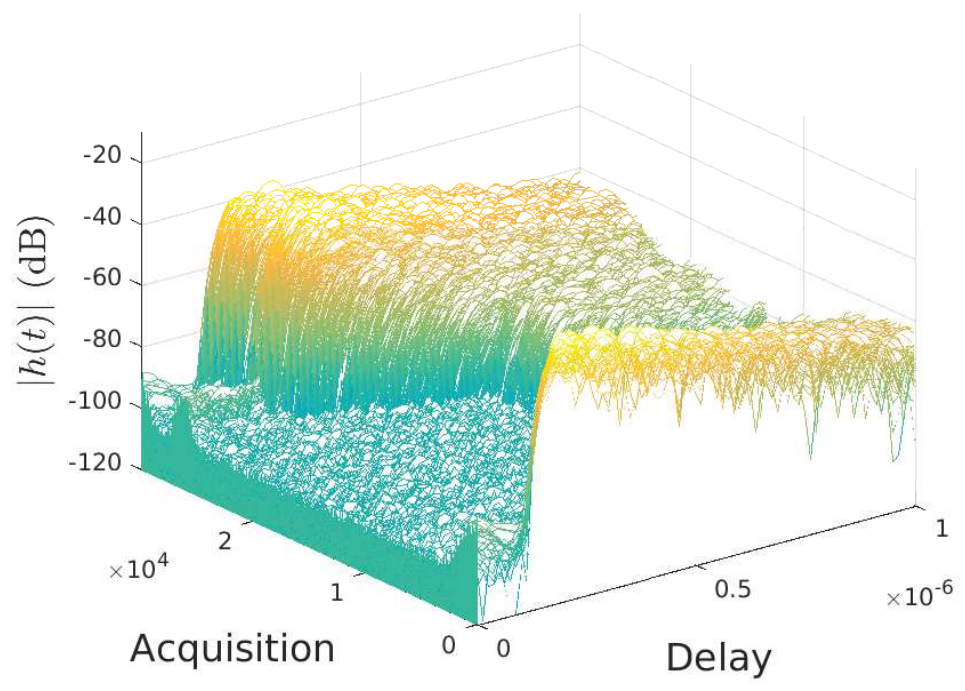

Figure 201: Impulse Responses: Horizontal Polarization, 5.4GHz, T2, Outer Path.

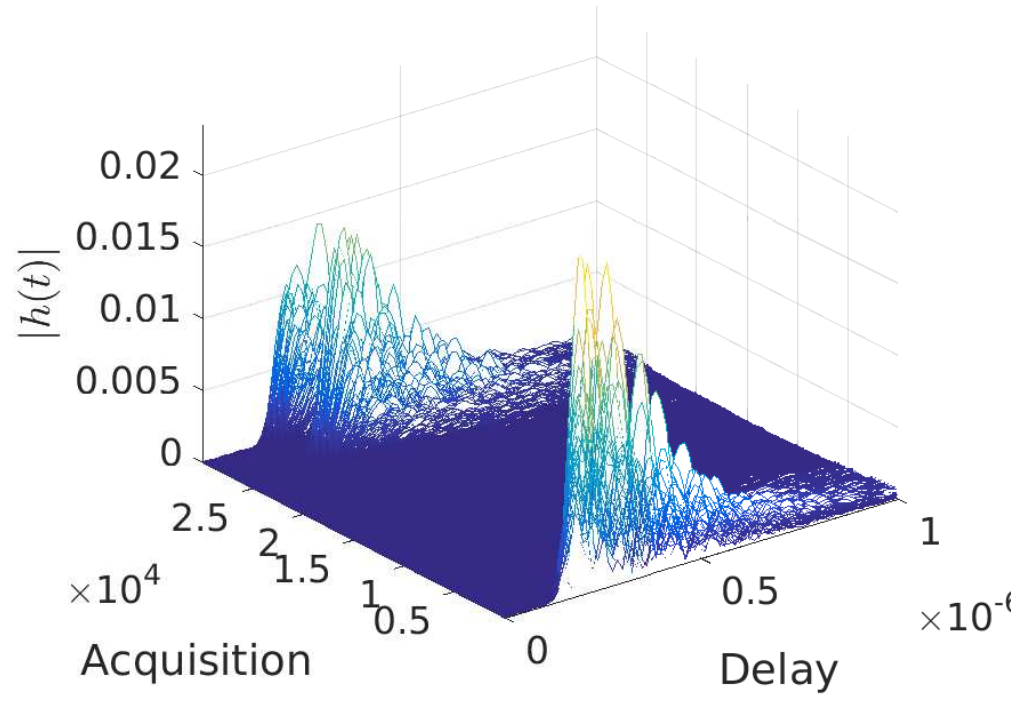

Figure 202: Impulse Responses: Horizontal Polarization, 5.4GHz, T2, Outer Path. 


\section{B.7 Horizontal Polarization, 2.245GHz, Transmitter 2, Inner Path}

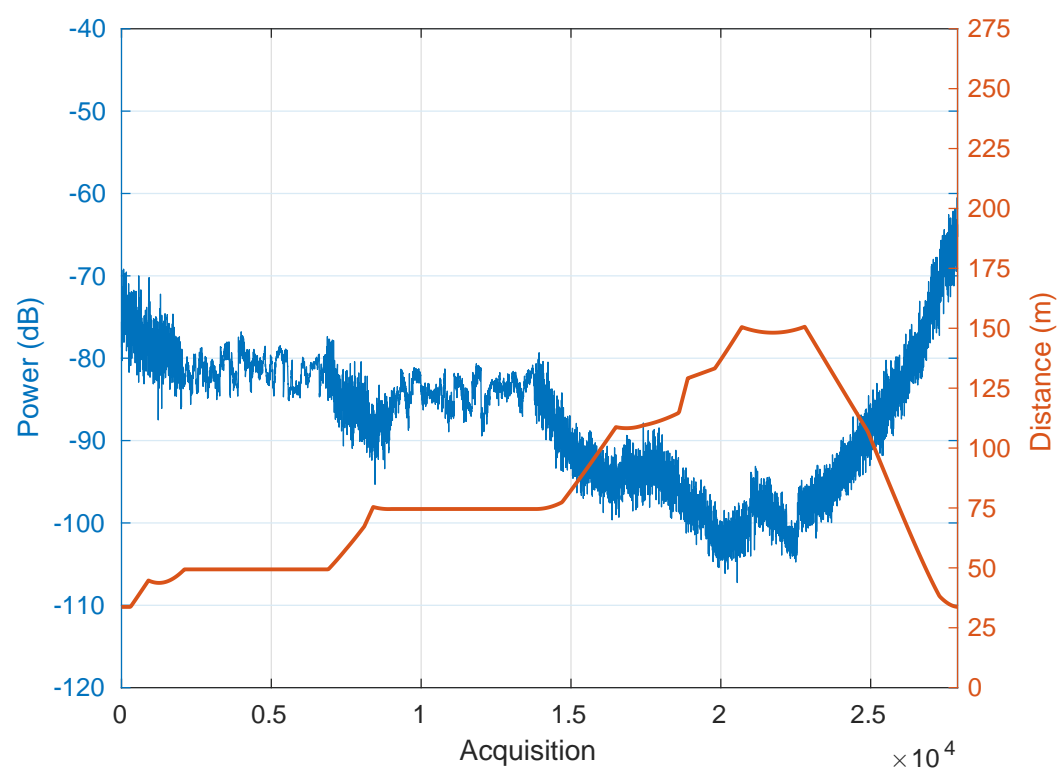

Figure 203: Overall Received Power vs. Acquisition Number and Distance vs. Acquisition Number: Horizontal Polarization, 2.245GHz, T2, Inner Path.

\begin{tabular}{c|c|c|c|c|c}
\hline Frequency & Polarization & $\alpha_{1}$ & $\alpha_{2}$ & $\beta(\mathrm{m})$ & $k_{1}(\mathrm{~dB})$ \\
\hline 3.2852 & 4.7235 & 74.5645 & -23.9979 & 2.9344 & 9.1289
\end{tabular}

Table 36: Parameters for Horizontal Polarization, 2.245GHz, T2, Inner Path 


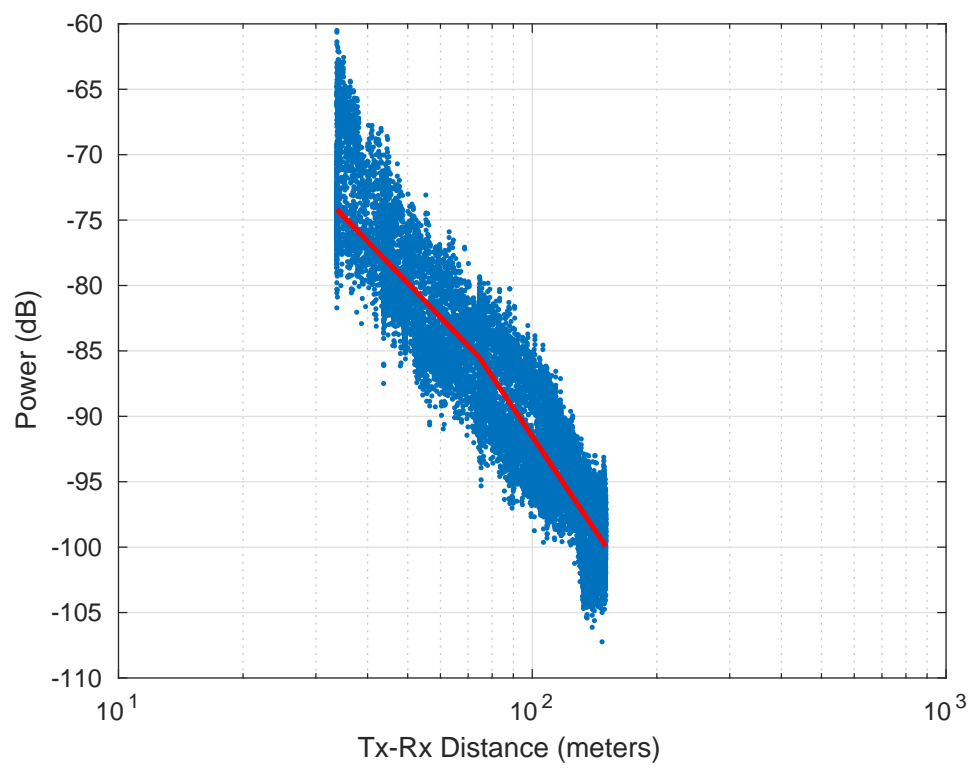

Figure 204: Overall Received Power vs. Distance: Horizontal Polarization, 2.245GHz, T2, Inner Path.

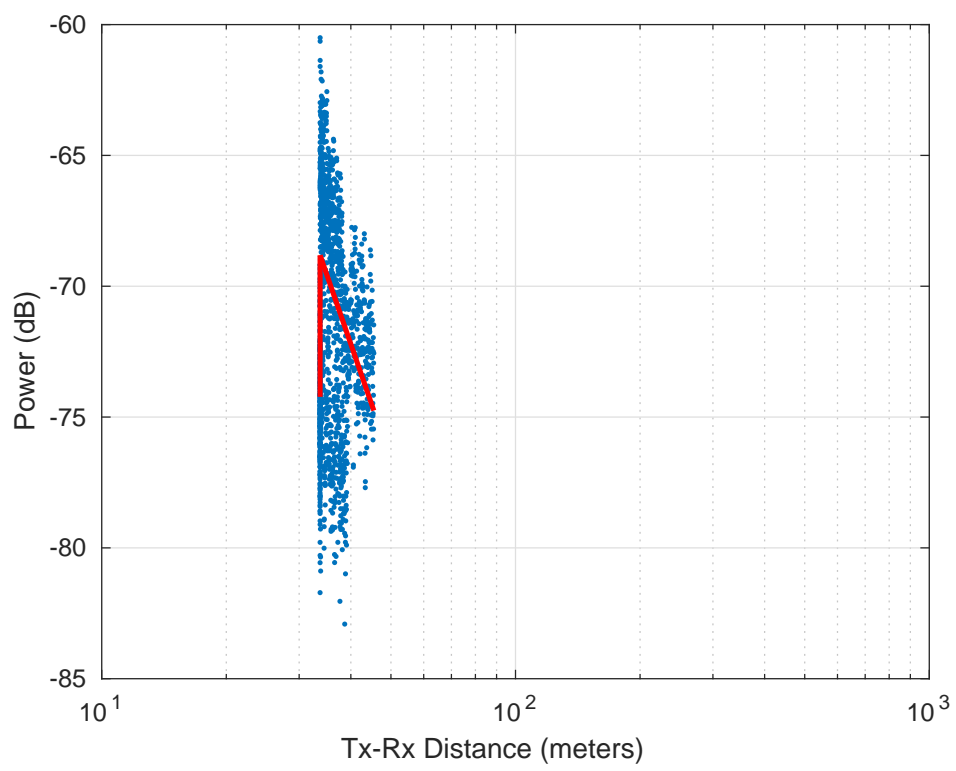

Figure 205: Line of Sight Received Power vs. Distance: Horizontal Polarization, $2.245 \mathrm{GHz}, \mathrm{T} 2$, Inner Path. 


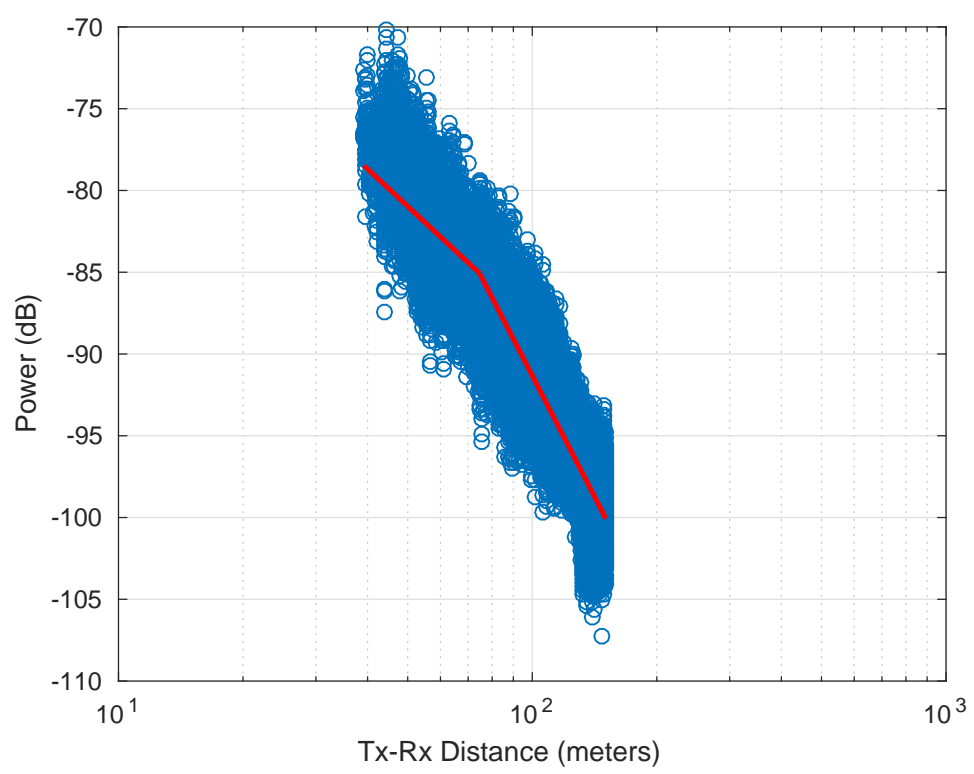

Figure 206: Non Line of Sight Received Power vs. Distance: Horizontal Polarization, $2.245 \mathrm{GHz}, \mathrm{T} 2$, Inner Path.

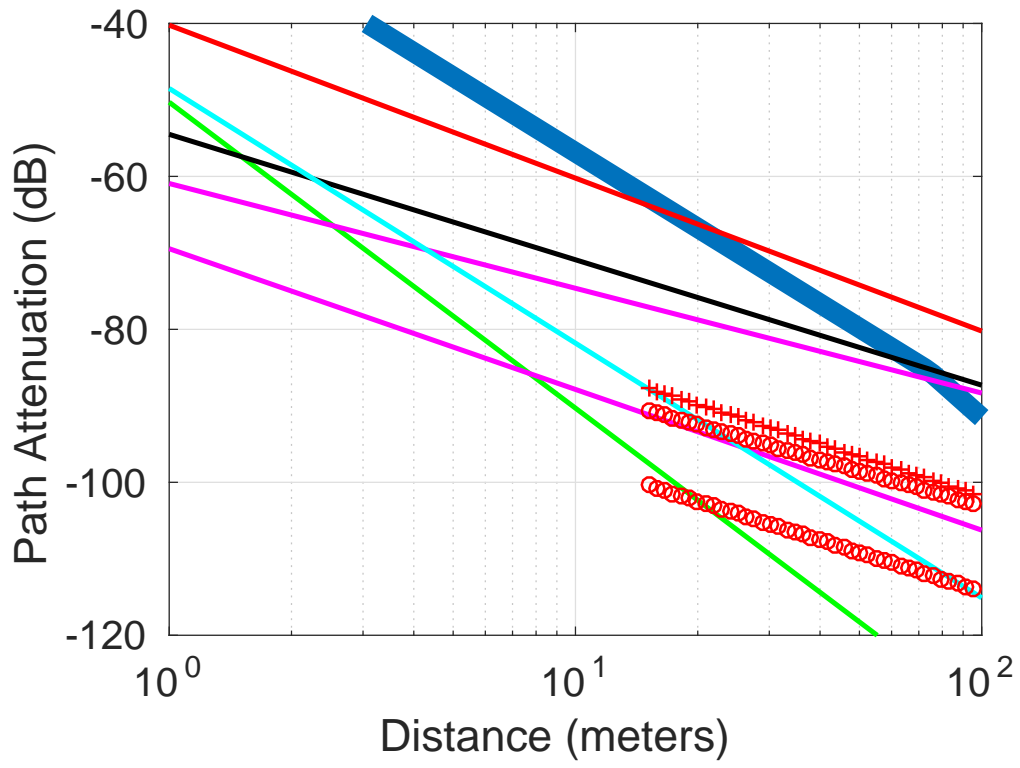

Figure 207: Comparison to other models: Horizontal Polarization, 2.245GHz, T2, Inner Path. 


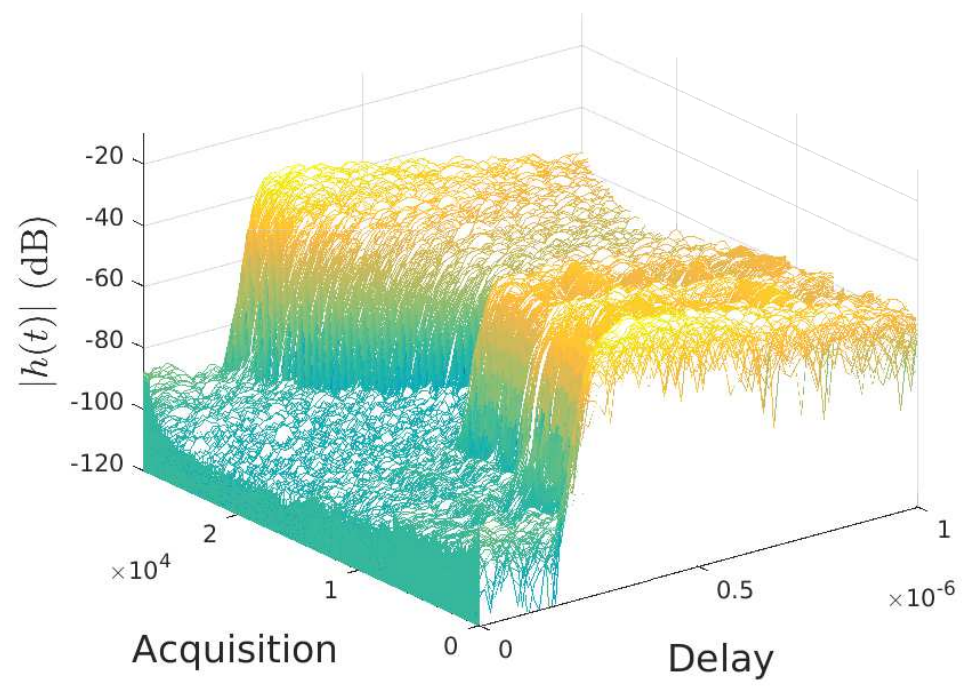

Figure 208: Impulse Responses: Horizontal Polarization, 2.245GHz, T2, Inner Path.

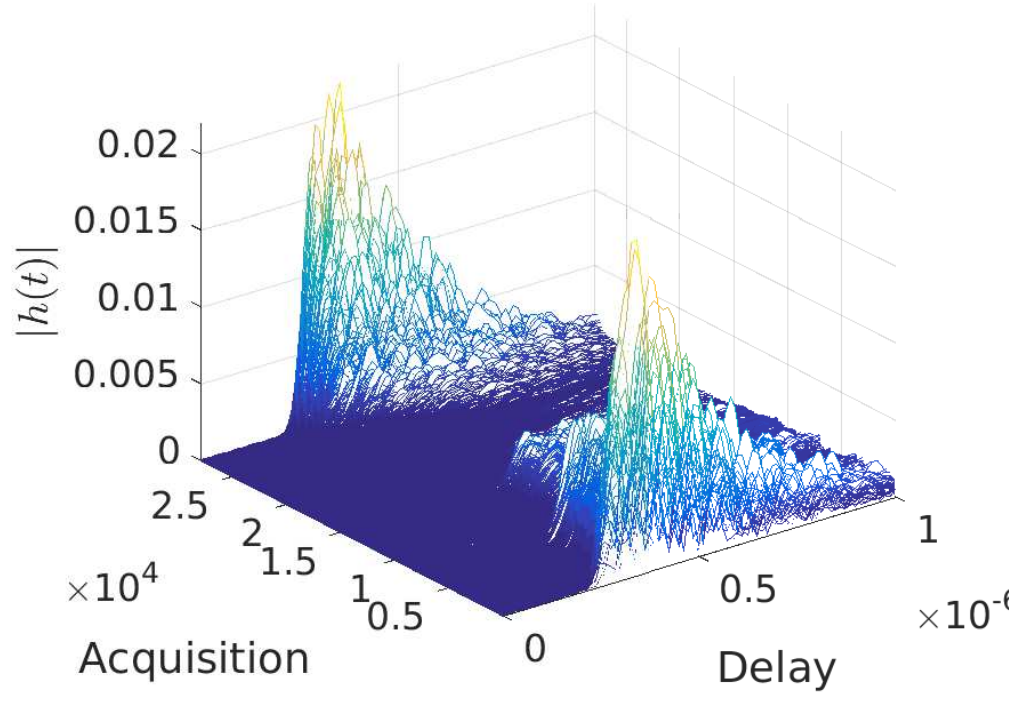

Figure 209: Impulse Responses: Horizontal Polarization, 2.245GHz, T2, Inner Path. 


\section{B.8 Horizontal Polarization, 5.4GHz, Transmitter 2, Inner Path}

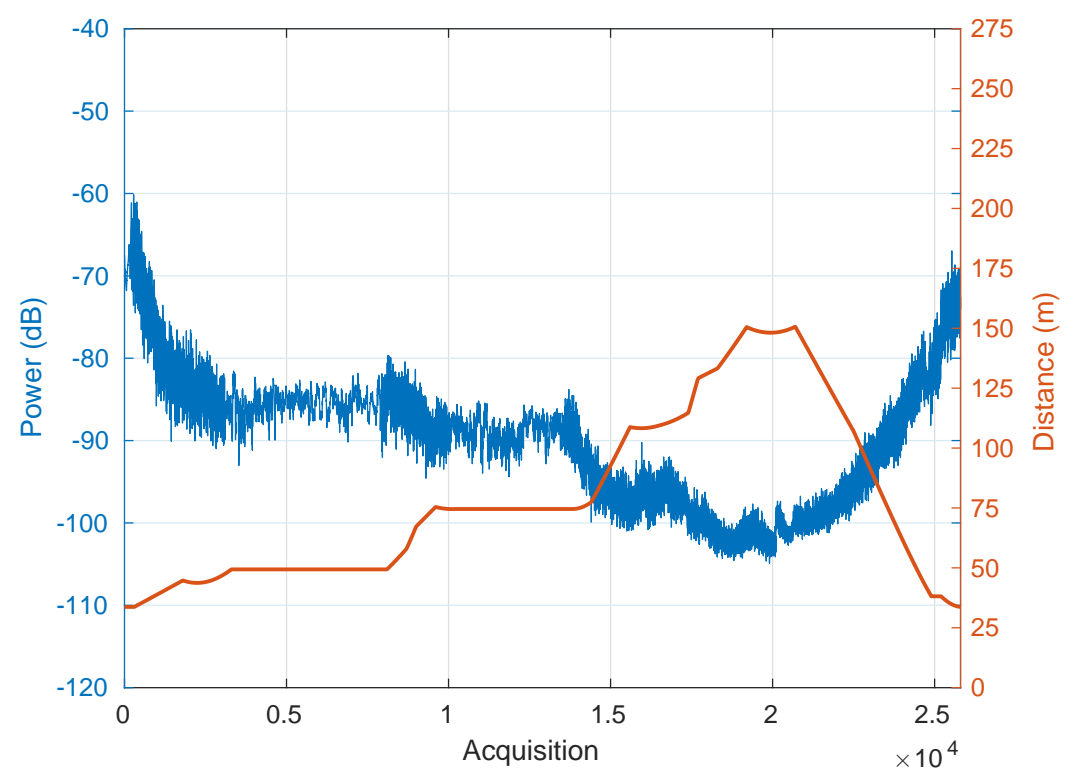

Figure 210: Overall Received Power vs. Acquisition Number and Distance vs. Acquisition Number: Horizontal Polarization, 5.4GHz, T2, Inner Path.

\begin{tabular}{c|c|c|c|c|c}
\hline Frequency & Polarization & $\alpha_{1}$ & $\alpha_{2}$ & $\beta(\mathrm{m})$ & $k_{1}(\mathrm{~dB})$ \\
\hline 15.8421 & 3.4173 & 40.3601 & 172.8361 & -26.7001 & 6.6621
\end{tabular}

Table 37: Parameters for Horizontal Polarization, 5.4GHz, T2, Inner Path 


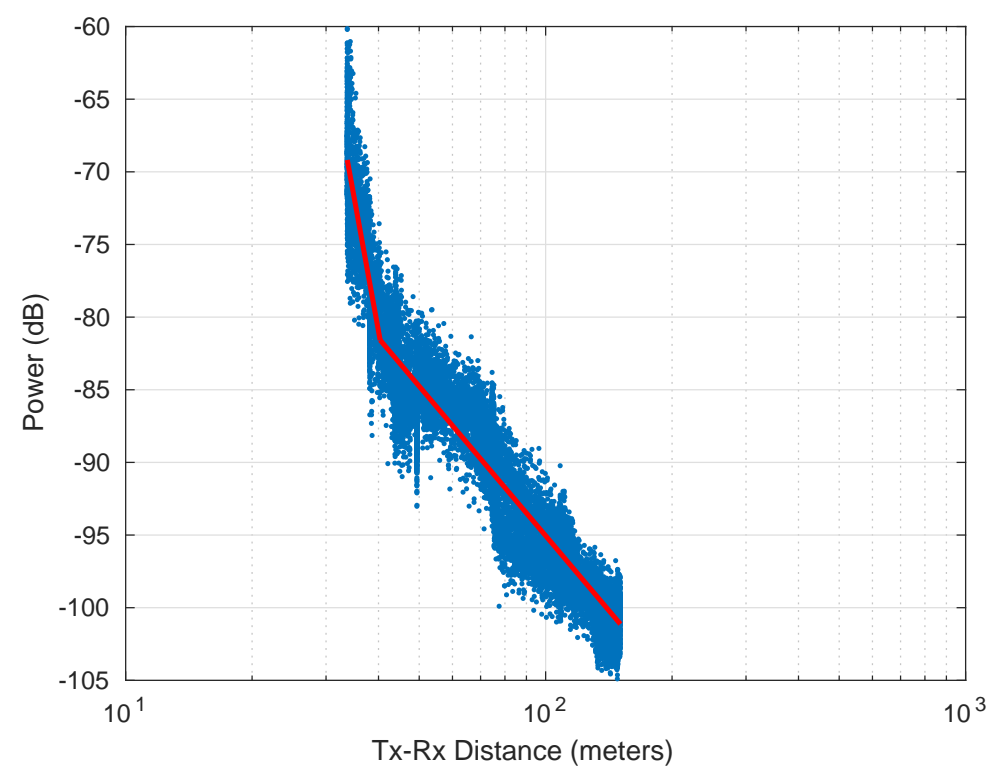

Figure 211: Overall Received Power vs. Distance: Horizontal Polarization, 5.4GHz, T2, Inner Path.

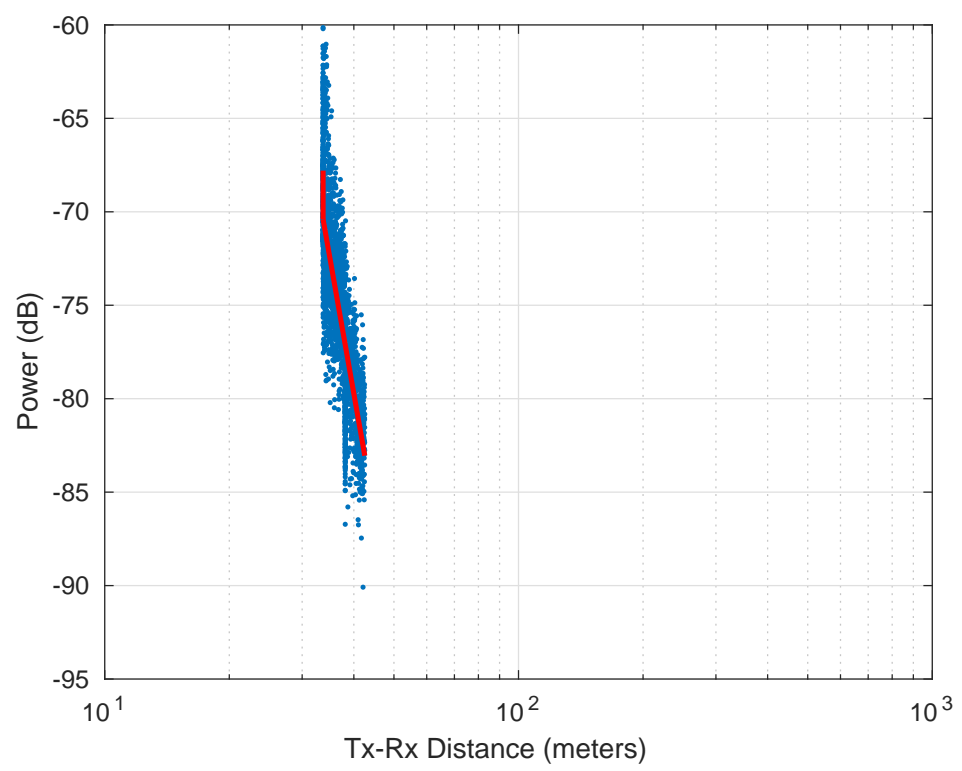

Figure 212: Line of Sight Received Power vs. Distance: Horizontal Polarization, 5.4GHz, T2, Inner Path. 


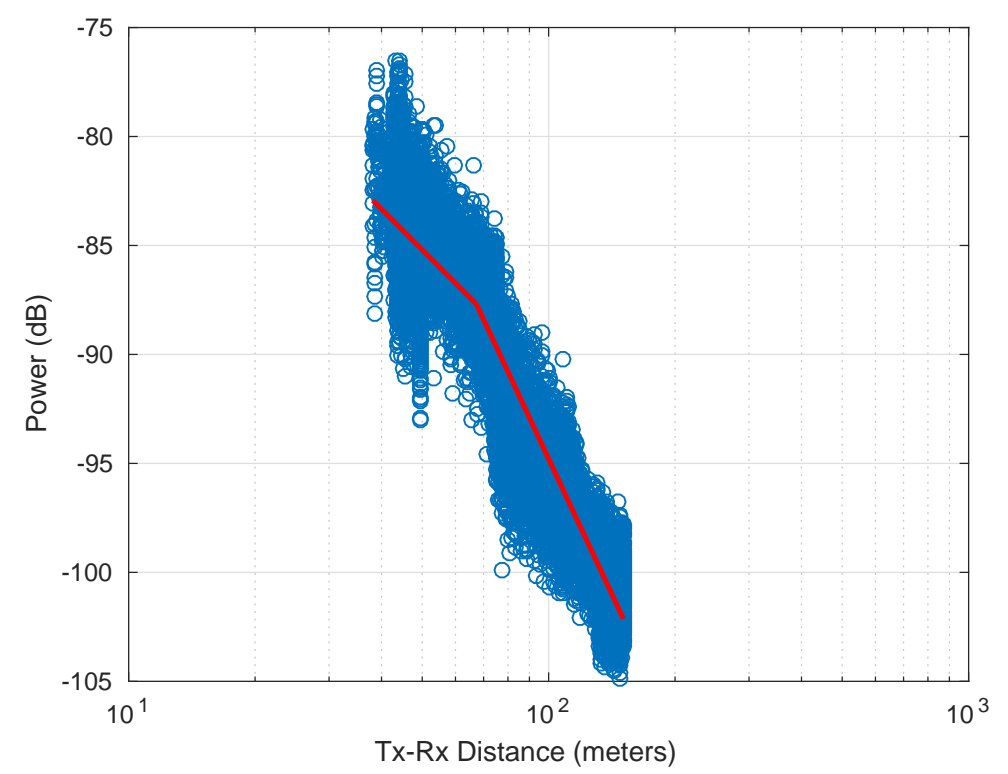

Figure 213: Non Line of Sight Received Power vs. Distance: Horizontal Polarization, 5.4GHz, T2, Inner Path.

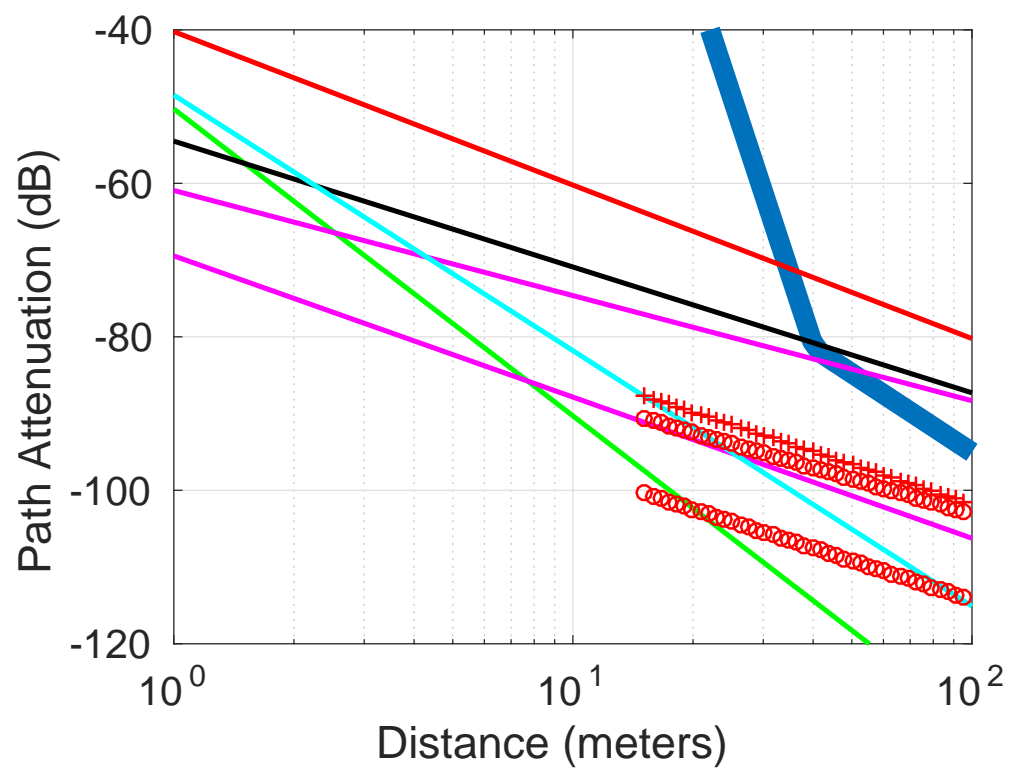

Figure 214: Comparison to other models: Horizontal Polarization, 5.4GHz, T2, Inner Path. 


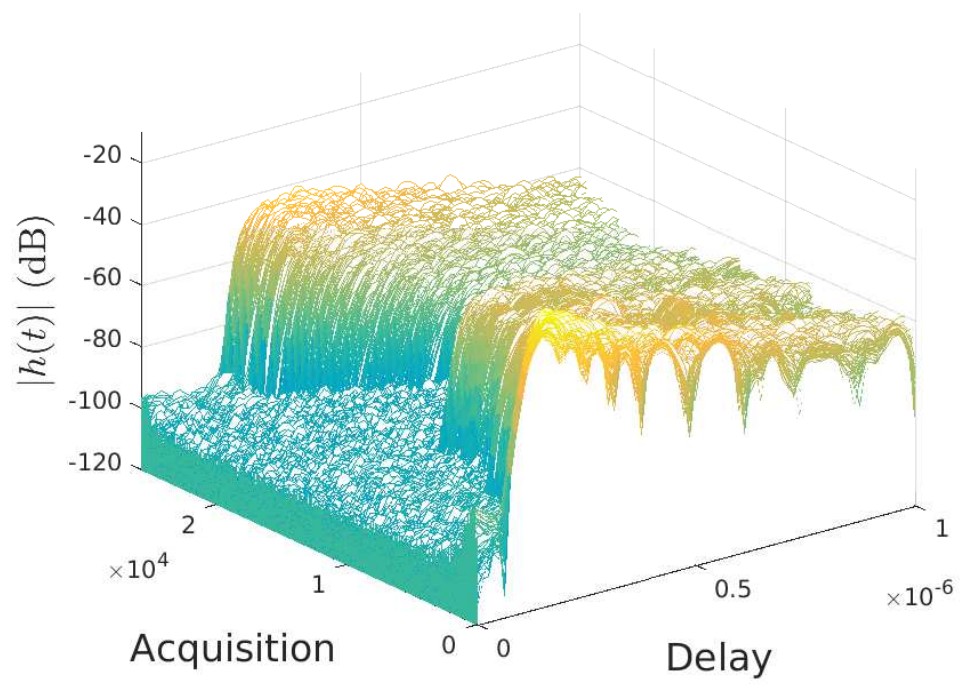

Figure 215: Impulse Responses: Horizontal Polarization, 5.4GHz, T2, Inner Path.

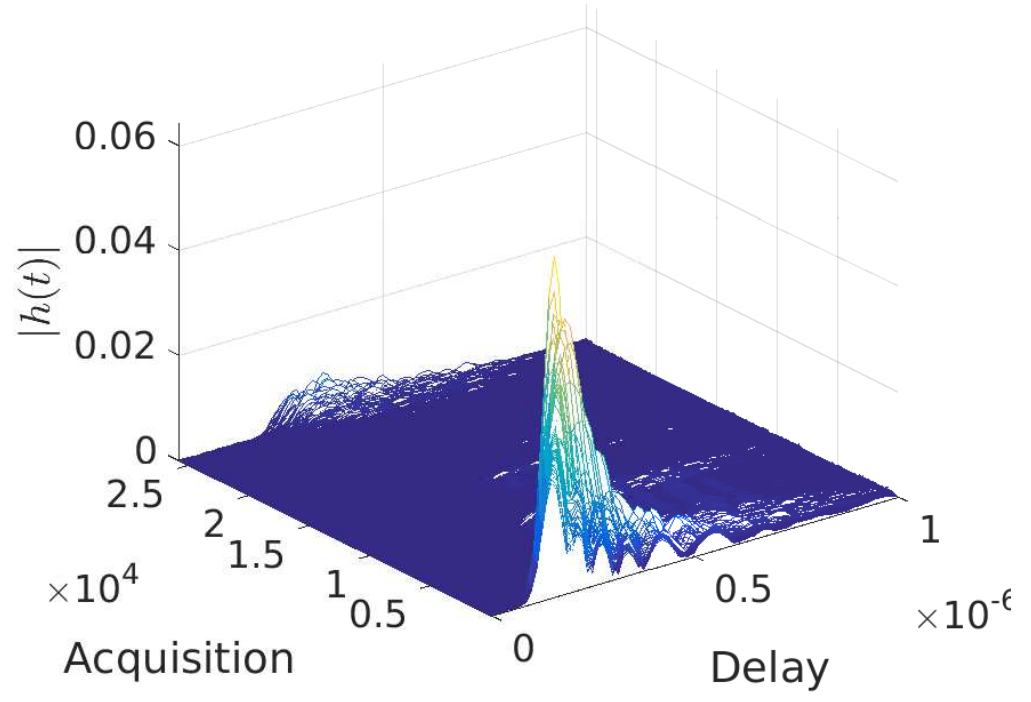

Figure 216: Impulse Responses: Horizontal Polarization, 5.4GHz, T2, Inner Path. 


\section{B.9 Vertical Polarization, 2.245GHz, Transmitter 1 Outer Path}

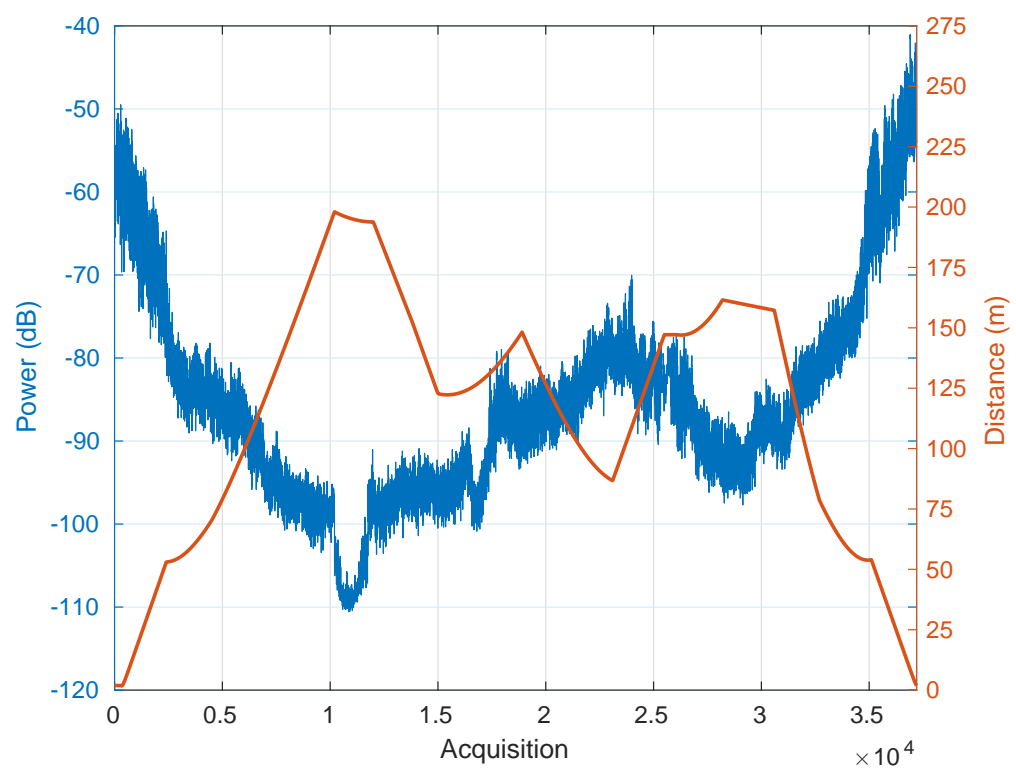

Figure 217: Overall Received Power vs. Acquisition Number and Distance vs. Acquisition Number: Vertical Polarization, 2.245GHz, T1, Outer Path.

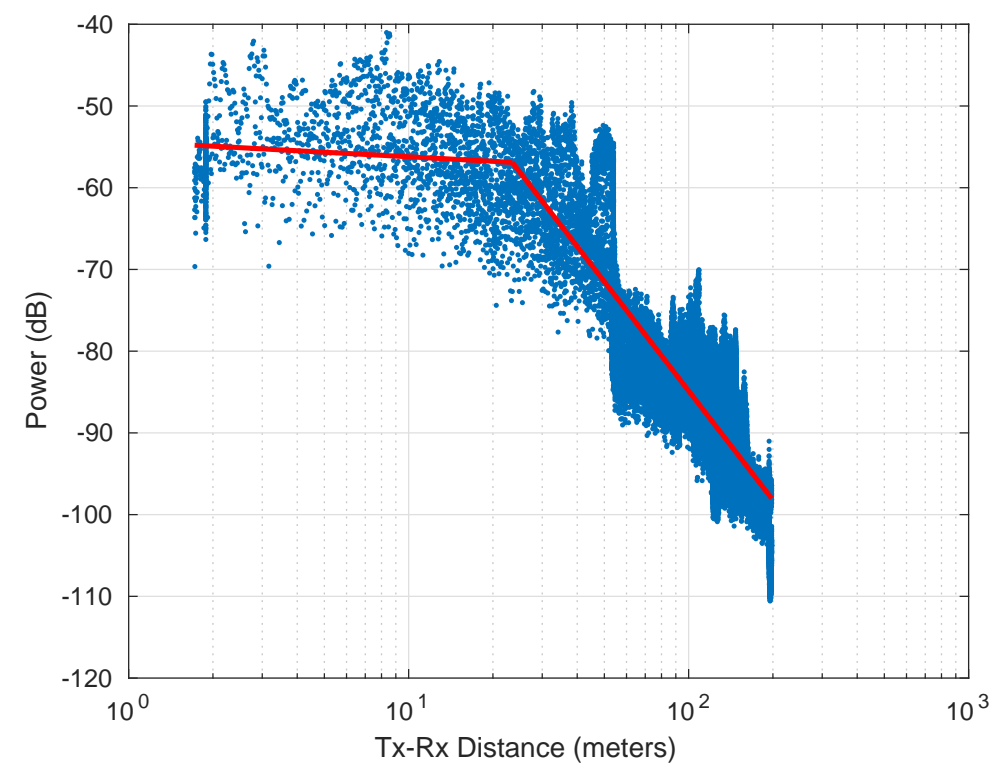

Figure 218: Overall Received Power vs. Distance: Vertical Polarization, 2.245GHz, T1, Outer Path. 


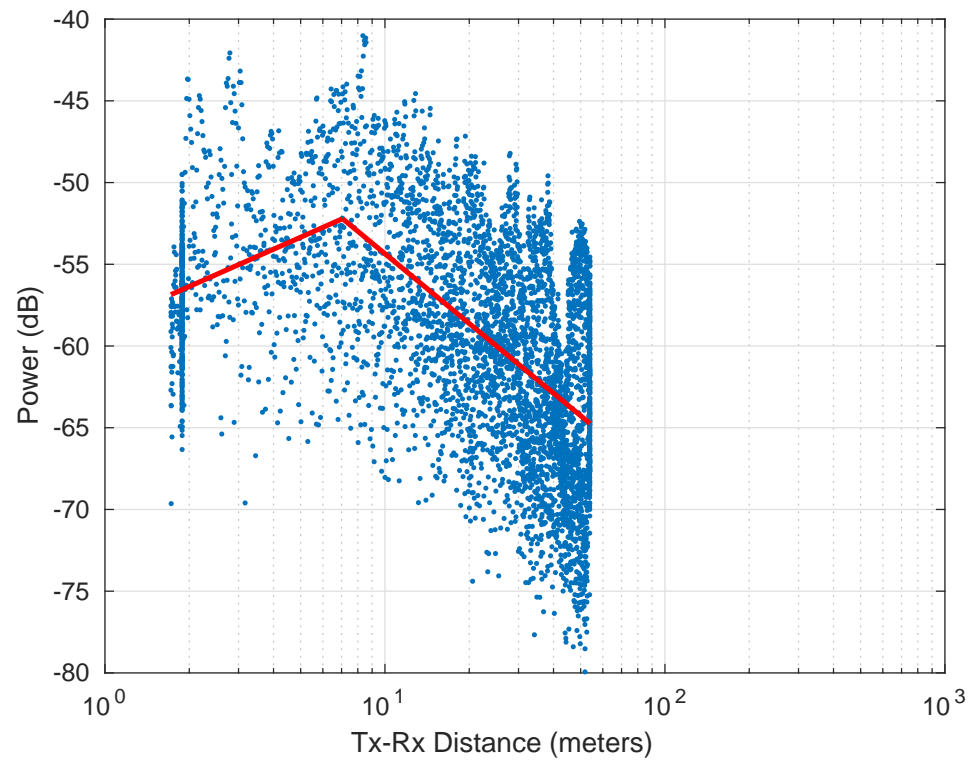

Figure 219: Line of Sight Received Power vs. Distance: Vertical Polarization, 2.245GHz, T1, Outer Path.

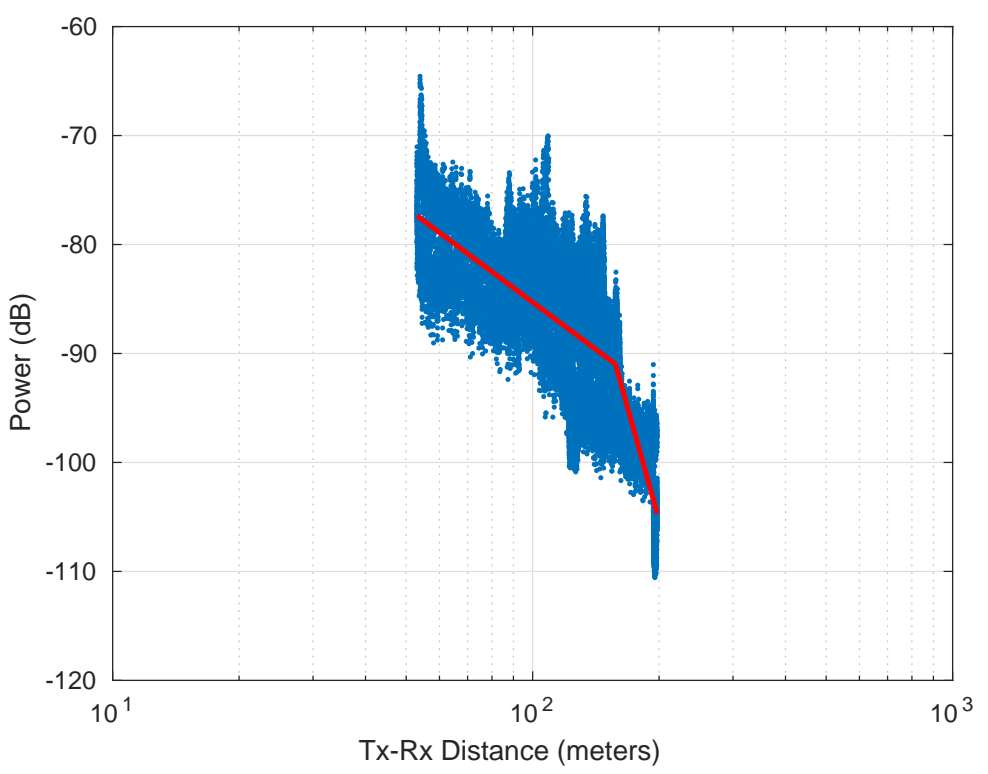

Figure 220: Non Line of Sight Received Power vs. Distance: Vertical Polarization, $2.245 \mathrm{GHz}, \mathrm{T} 1$, Outer Path.

\begin{tabular}{c|c|c|c|c|c}
\hline$\alpha_{1}$ & $\alpha_{2}$ & $\beta(\mathrm{m})$ & $k_{1}(\mathrm{~dB})$ & $k_{2}(\mathrm{~dB})$ & MSE \\
\hline 0.18534 & 4.4378 & 23.4457 & -54.3605 & 3.9013 & 14.9937
\end{tabular}

Table 38: Parameters for Vertical Polarization, 2.245GHz, T1, Outer Path 


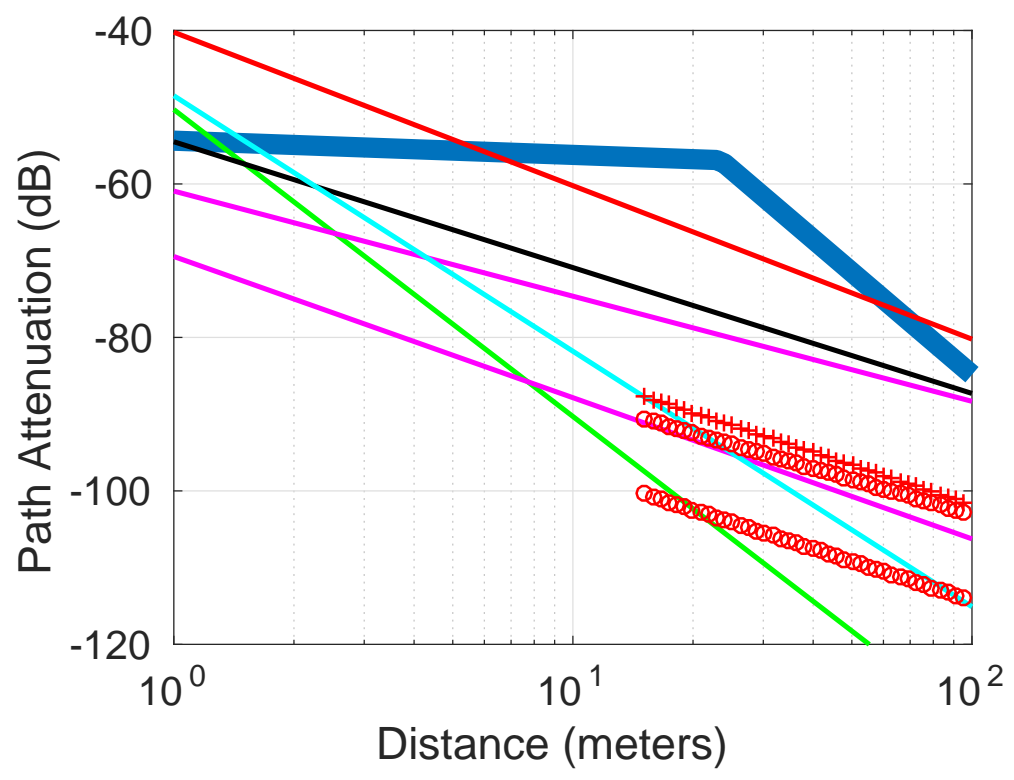

Figure 221: Comparison to other models: Vertical Polarization, 2.245GHz, T1, Outer Path.

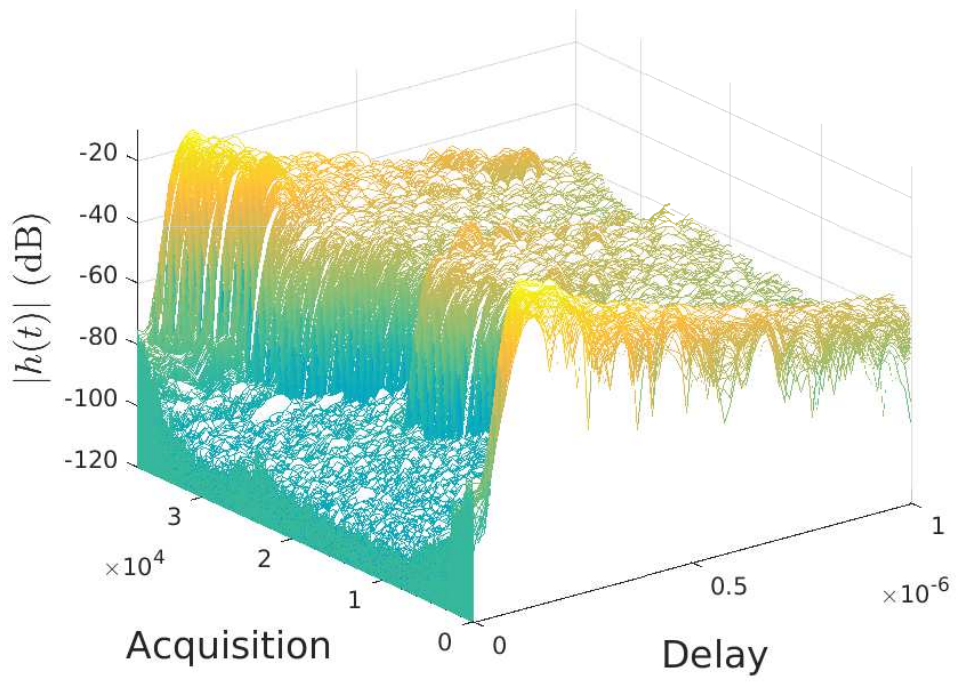

Figure 222: Impulse Responses: Vertical Polarization, 2.245GHz, T1, Outer Path. 


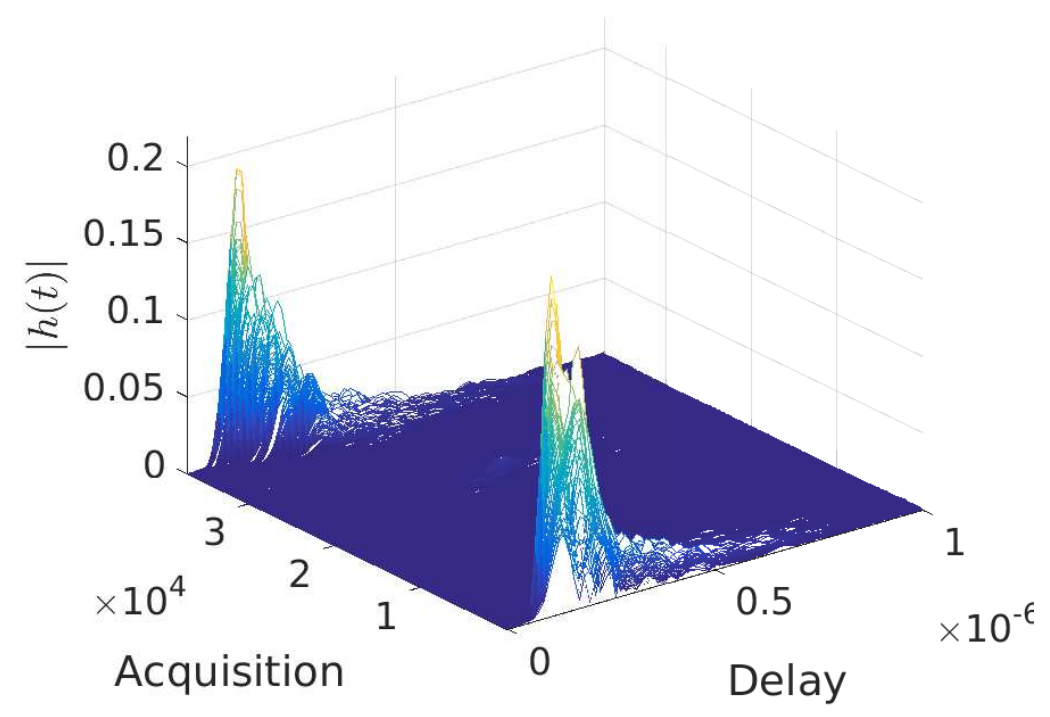

Figure 223: Impulse Responses: Vertical Polarization, 2.245GHz, T1, Outer Path. 
B.10 Vertical Polarization, 5.4GHz, Transmitter 1, Outer Path

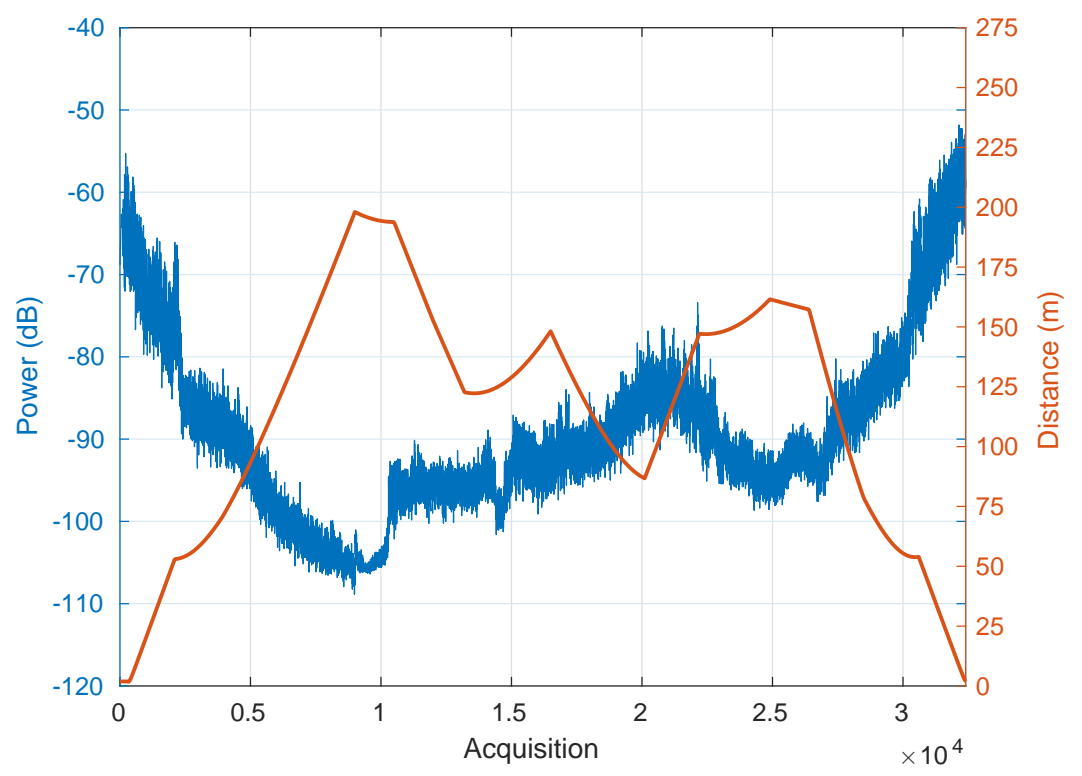

Figure 224: Overall Received Power vs. Acquisition Number and Distance vs. Acquisition Number: Vertical Polarization, 5.4GHz, T1, Outer Path.

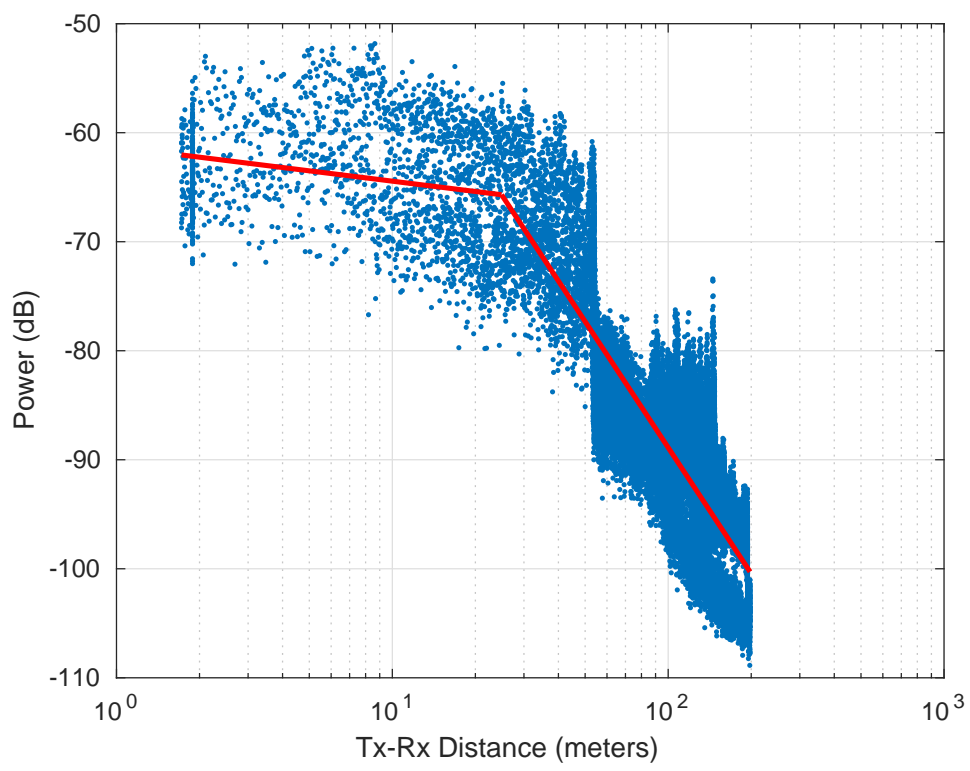

Figure 225: Overall Received Power vs. Distance: Vertical Polarization, 5.4GHz, T1, Outer Path. 


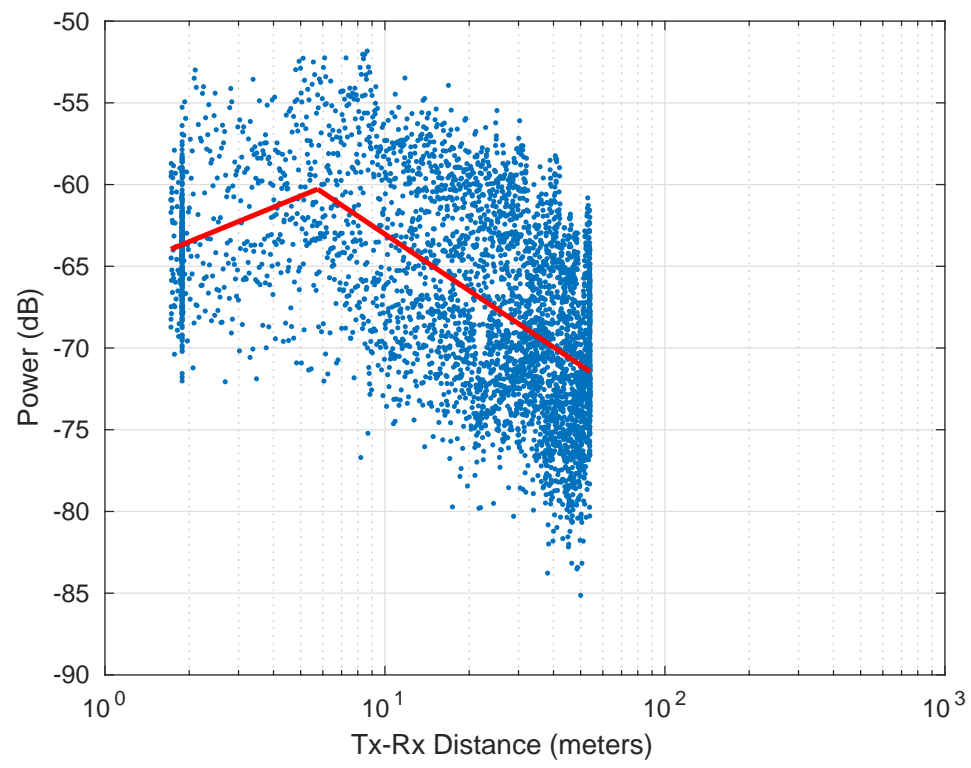

Figure 226: Line of Sight Received Power vs. Distance: Vertical Polarization, 5.4GHz, T1, Outer Path.

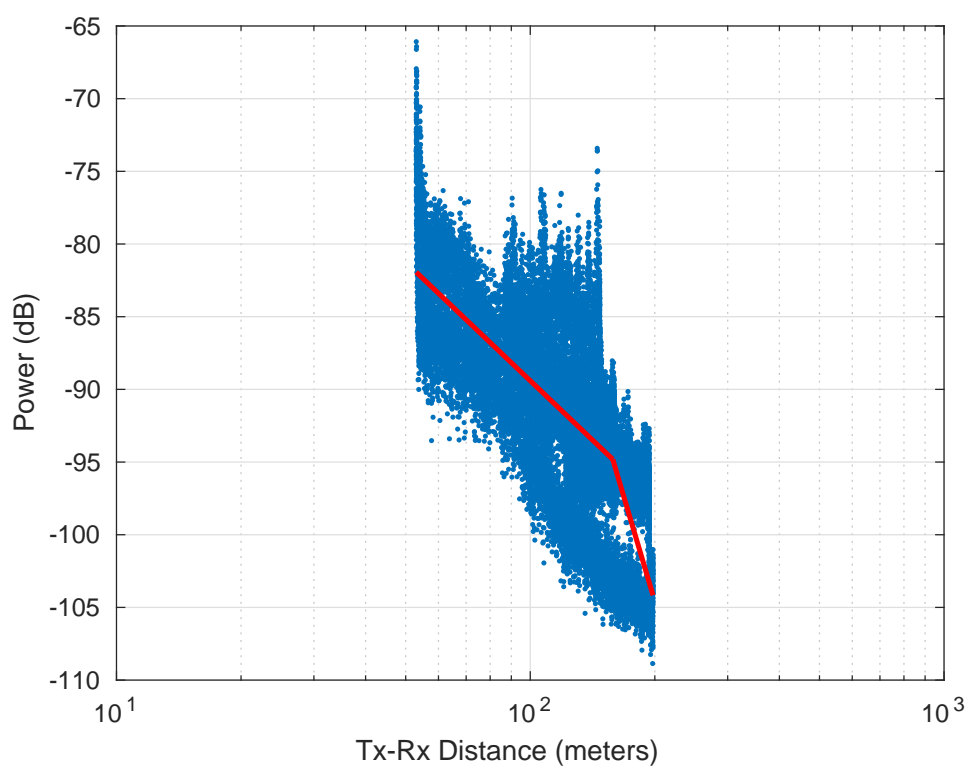

Figure 227: Non Line of Sight Received Power vs. Distance: Vertical Polarization, $5.4 \mathrm{GHz}, \mathrm{T} 1$, Outer Path.

\begin{tabular}{c|c|c|c|c|c}
\hline$\alpha_{1}$ & $\alpha_{2}$ & $\beta(\mathrm{m})$ & $k_{1}(\mathrm{~dB})$ & $k_{2}(\mathrm{~dB})$ & MSE \\
\hline 0.31471 & 3.8395 & 24.888 & -61.2997 & -12.0941 & 13.7568
\end{tabular}

Table 39: Parameters for Vertical Polarization, 5.4GHz, T1, Outer Path 


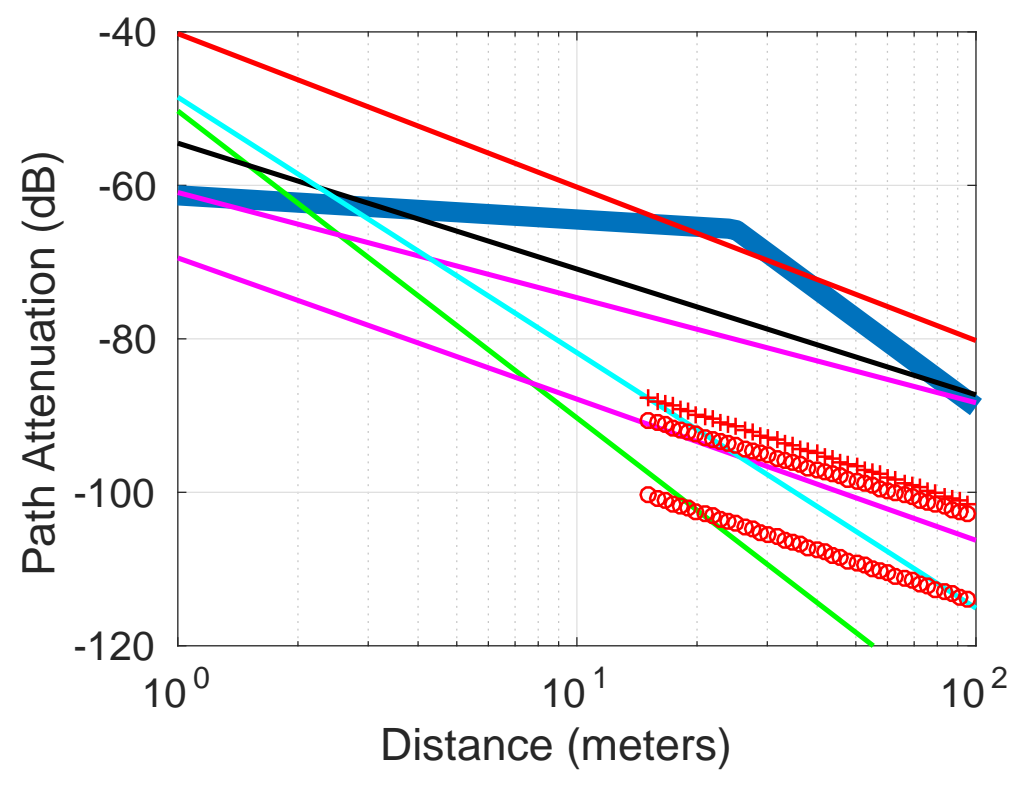

Figure 228: Comparison to other models: Vertical Polarization, 5.4GHz, T1, Outer Path.

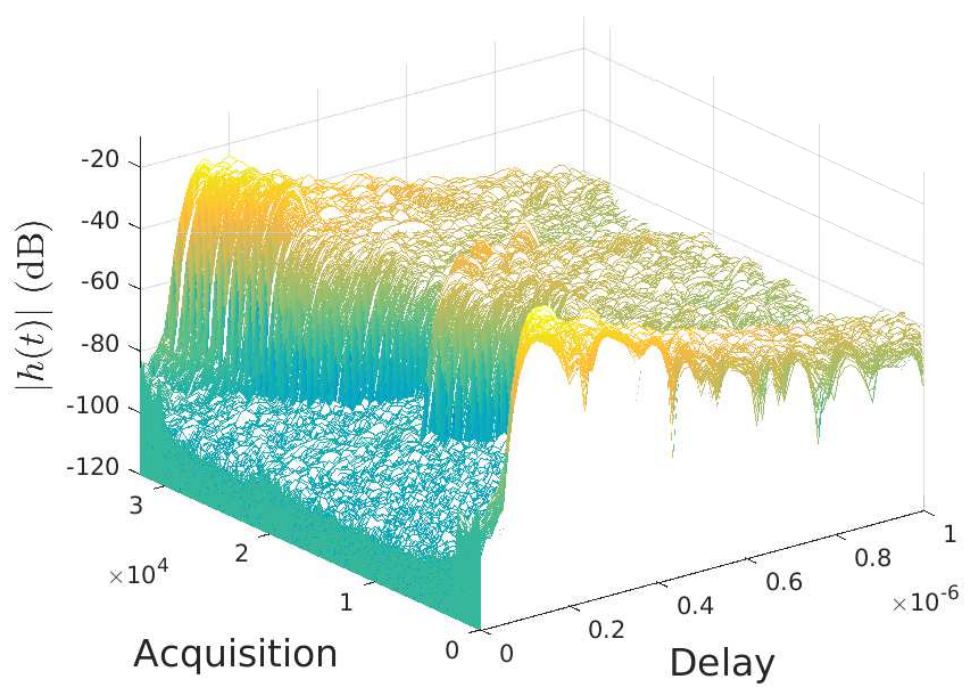

Figure 229: Impulse Responses: Vertical Polarization, 5.4GHz, T1, Outer Path. 


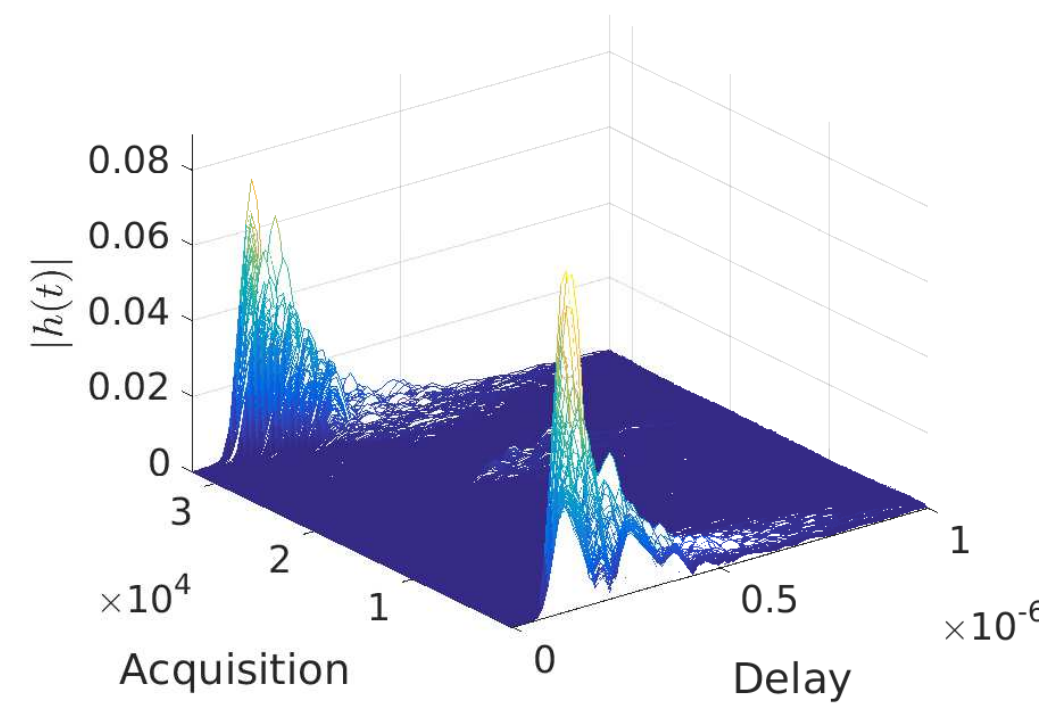

Figure 230: Impulse Responses: Vertical Polarization, 5.4GHz, T1, Outer Path. 


\section{B.11 Vertical Polarization, 2.245GHz, Transmitter 1, Inner Path}

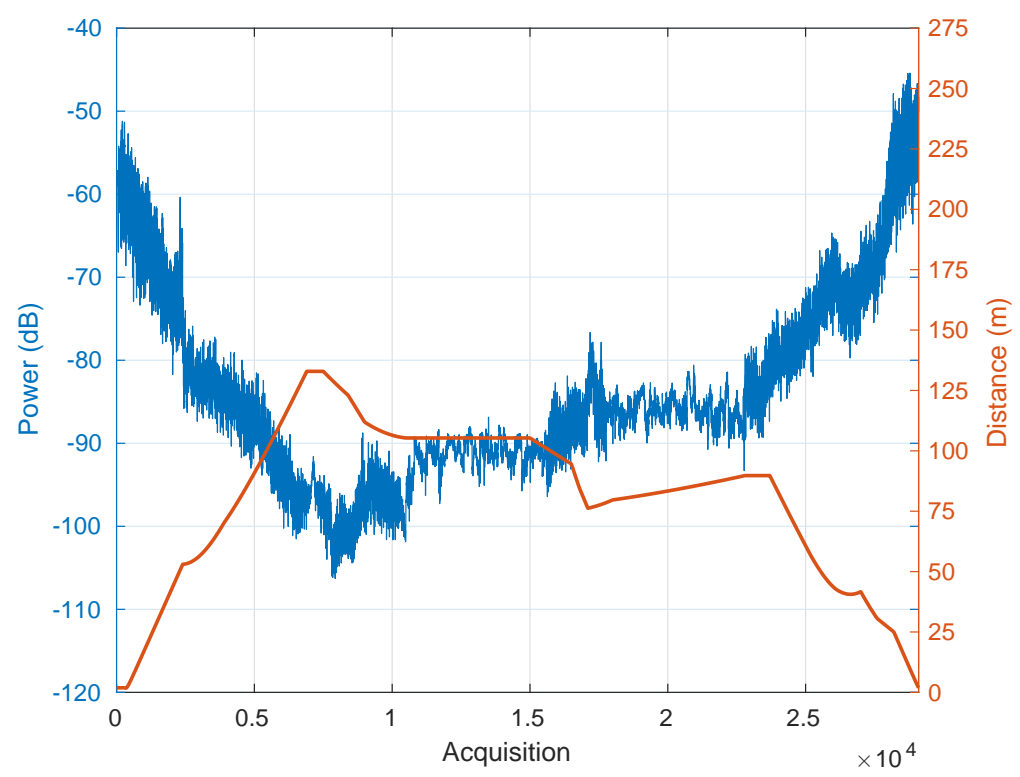

Figure 231: Overall Received Power vs. Acquisition Number and Distance vs. Acquisition Number: Vertical Polarization, 2.245GHz, T1, Inner Path.

\begin{tabular}{c|c|c|c|c|c}
\hline$\alpha_{1}$ & $\alpha_{2}$ & $\beta(\mathrm{m})$ & $k_{1}(\mathrm{~dB})$ & $k_{2}(\mathrm{~dB})$ & MSE \\
\hline 0.16754 & 5.0896 & 23.88 & -56.5334 & 11.2937 & 10.8275
\end{tabular}

Table 40: Parameters for Vertical Polarization, 2.245GHz, T1, Inner Path 


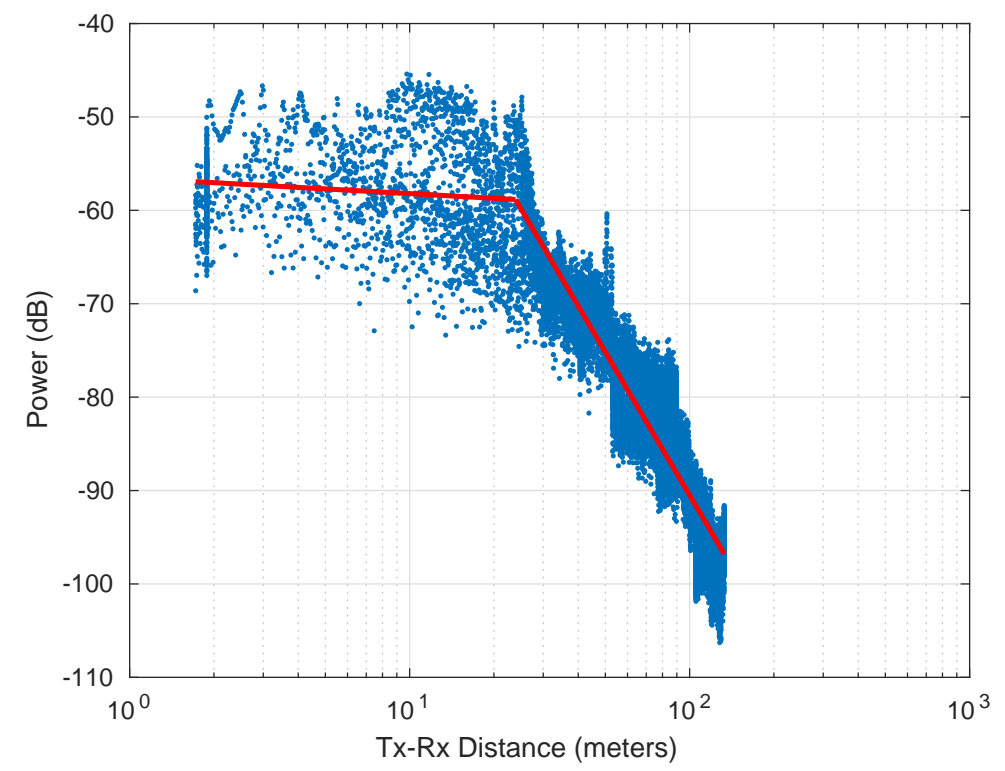

Figure 232: Overall Received Power vs. Distance: Vertical Polarization, 2.245GHz, T1, Inner Path.

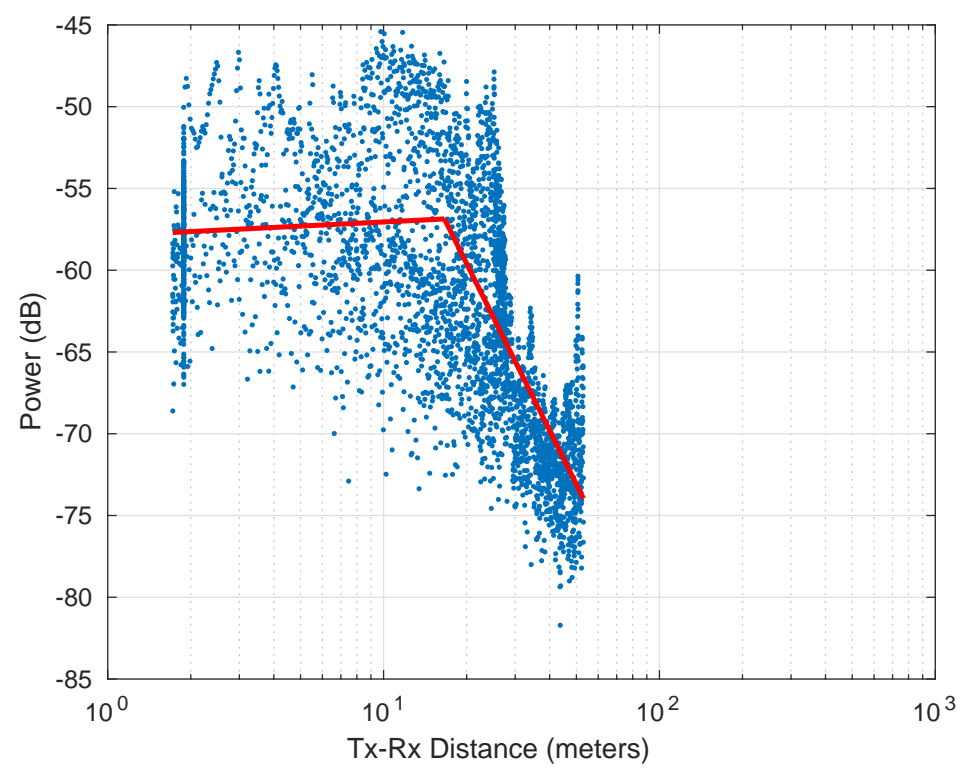

Figure 233: Line of Sight Received Power vs. Distance: Vertical Polarization, 2.245GHz, T1, Inner Path. 


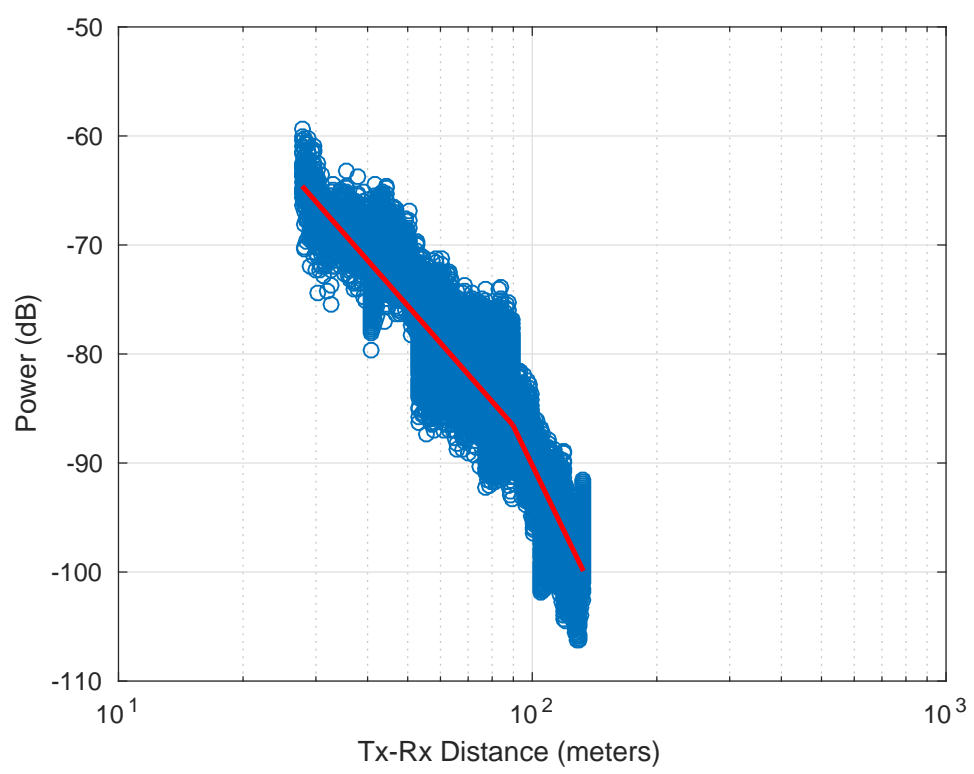

Figure 234: Non Line of Sight Received Power vs. Distance: Vertical Polarization, $2.245 \mathrm{GHz}, \mathrm{T} 1$, Inner Path.

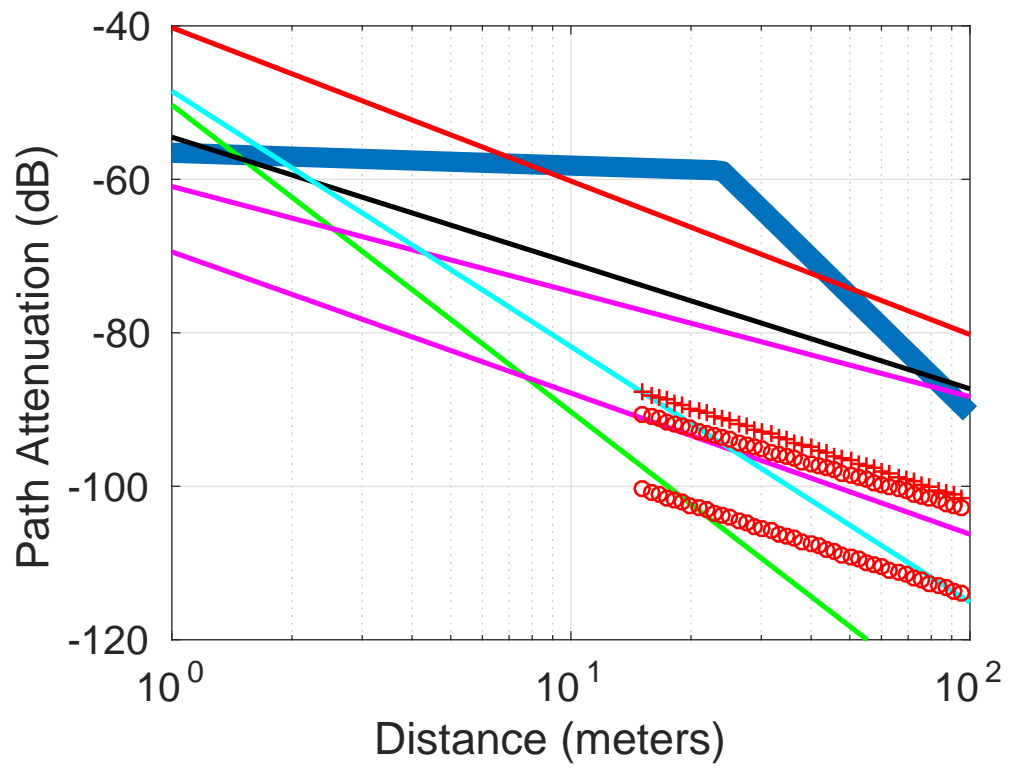

Figure 235: Comparison to other models: Vertical Polarization, 2.245GHz, T1, Inner Path. 


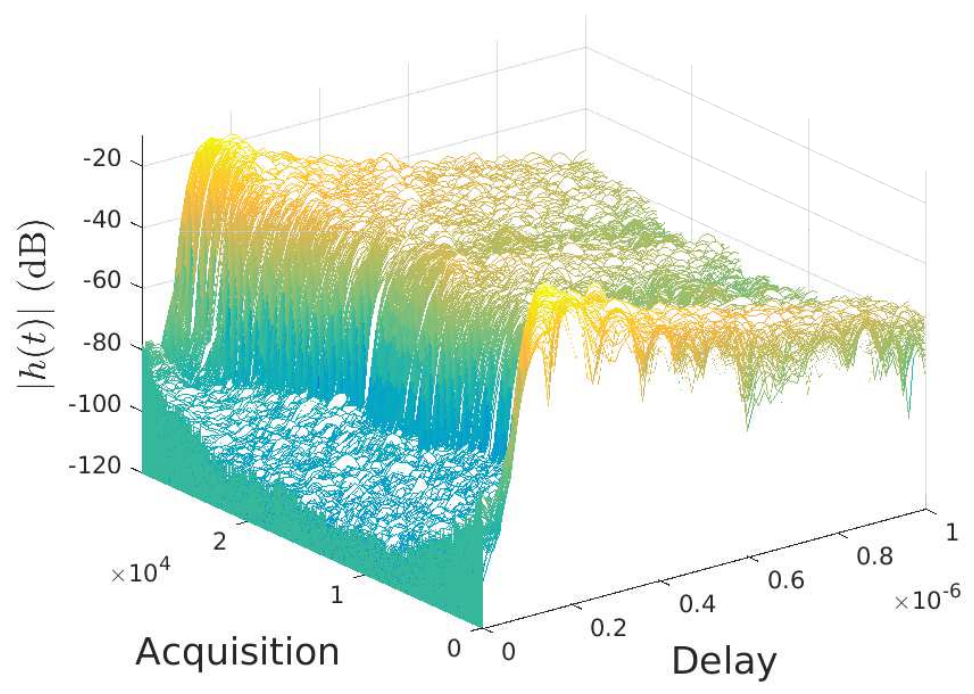

Figure 236: Impulse Responses: Vertical Polarization, 2.245GHz, T1, Inner Path.

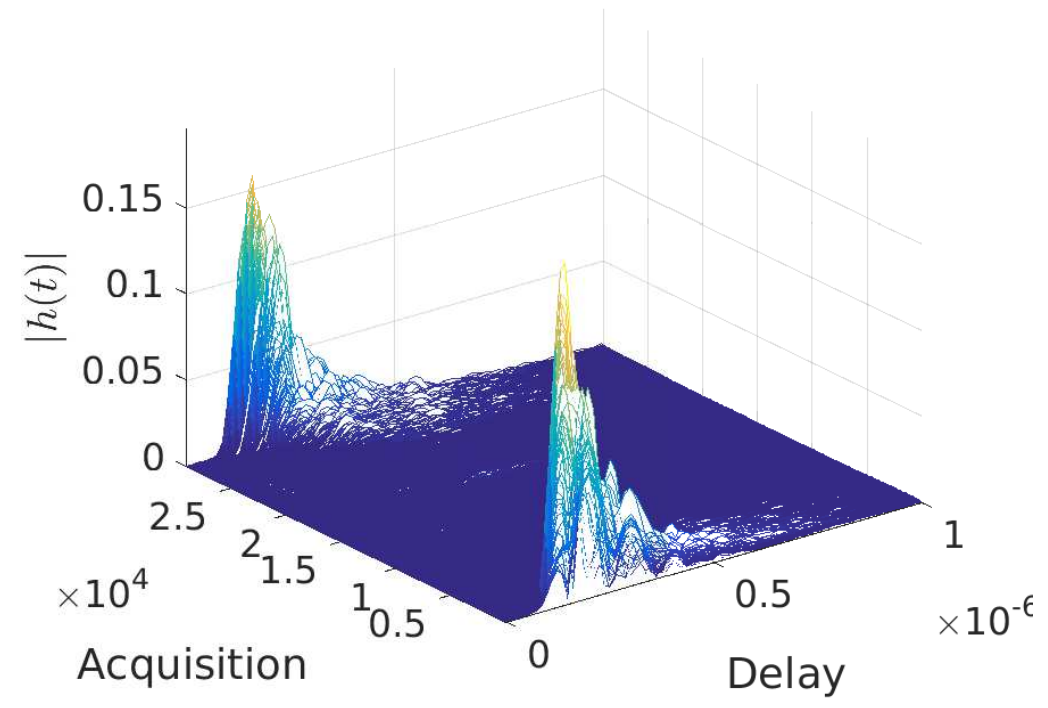

Figure 237: Impulse Responses: Vertical Polarization, 2.245GHz, T1, Inner Path. 


\section{B.12 Vertical Polarization, 5.4GHz, Transmitter 1, Inner Path}

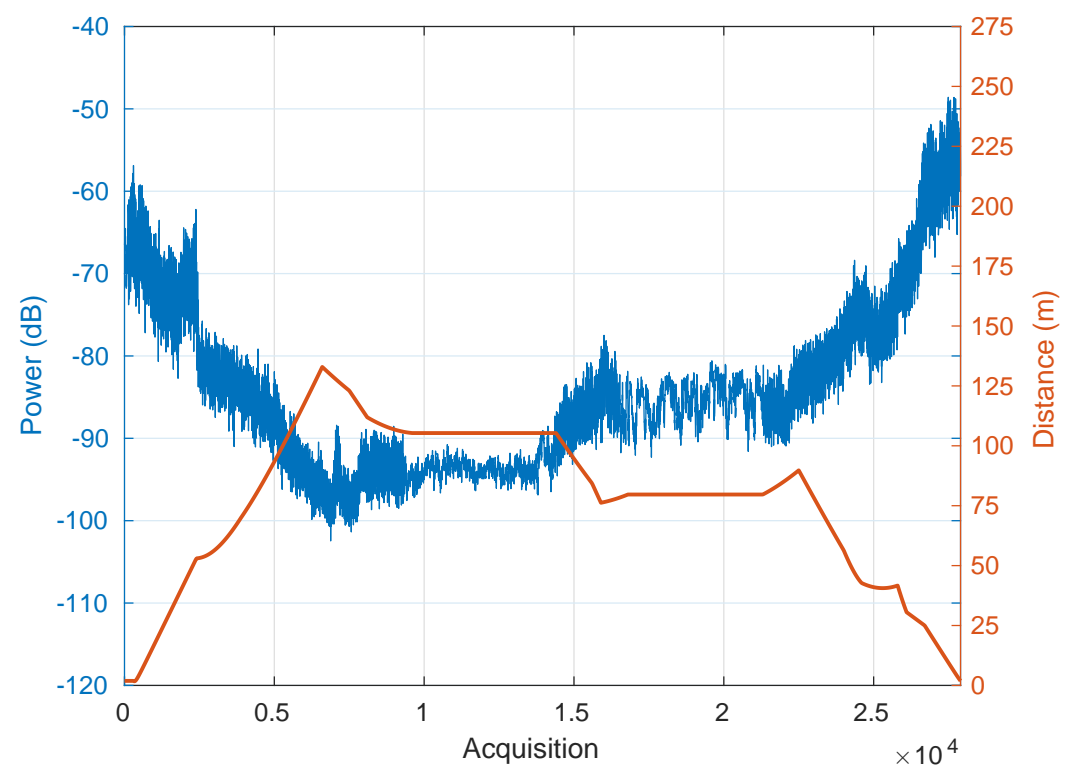

Figure 238: Overall Received Power vs. Acquisition Number and Distance vs. Acquisition Number: Vertical Polarization, 5.4GHz, T1, Inner Path.

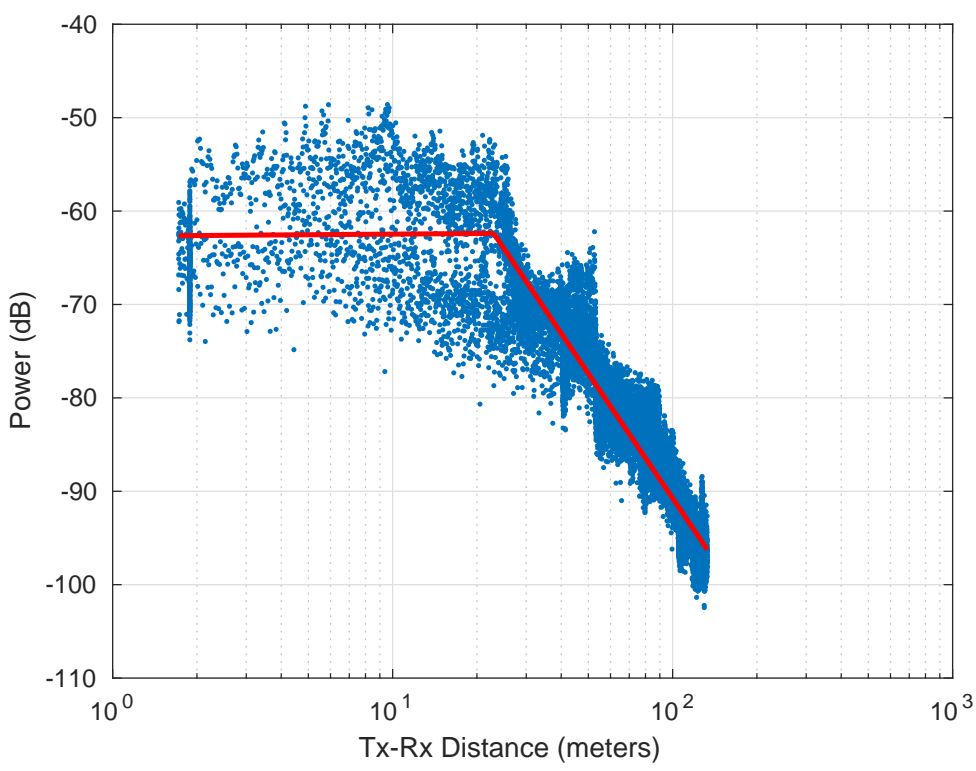

Figure 239: Overall Received Power vs. Distance: Vertical Polarization, 5.4GHz, T1, Inner Path. 


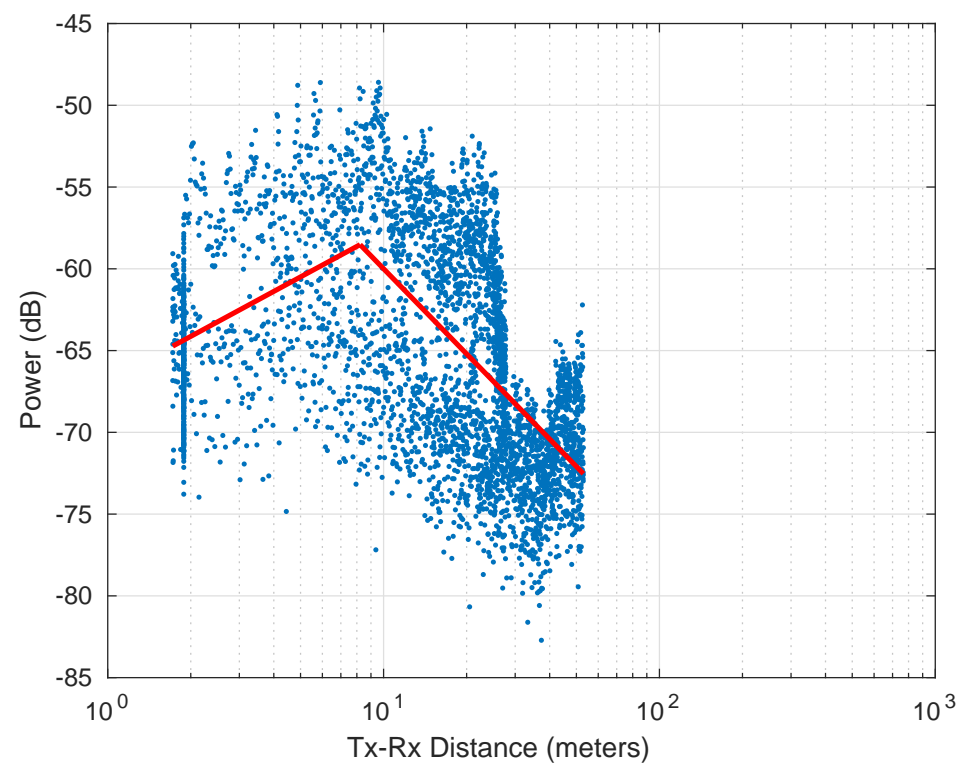

Figure 240: Line of Sight Received Power vs. Distance: Vertical Polarization, 5.4GHz, T1, Inner Path.

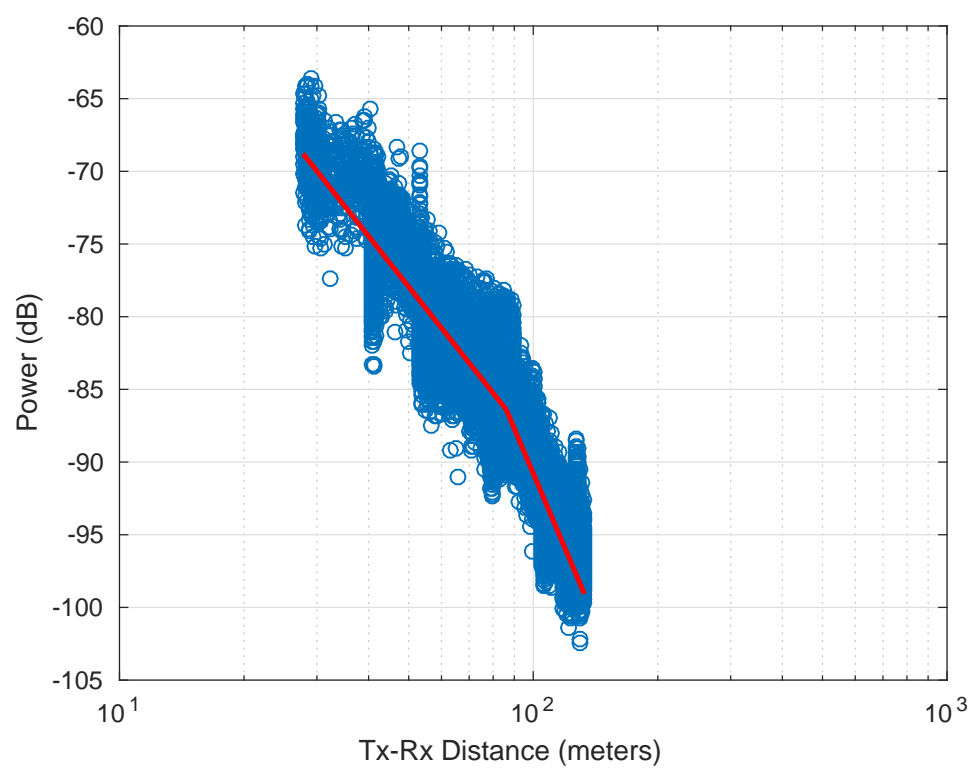

Figure 241: Non Line of Sight Received Power vs. Distance: Vertical Polarization, 5.4GHz, T1, Inner Path.

\begin{tabular}{c|c|c|c|c|c}
\hline$\alpha_{1}$ & $\alpha_{2}$ & $\beta(\mathrm{m})$ & $k_{1}(\mathrm{~dB})$ & $k_{2}(\mathrm{~dB})$ & MSE \\
\hline-0.021878 & 4.4378 & 22.9532 & -62.6865 & -1.9973 & 10.3104
\end{tabular}

Table 41: Parameters for Vertical Polarization, 5.4GHz, T1, Inner Path 


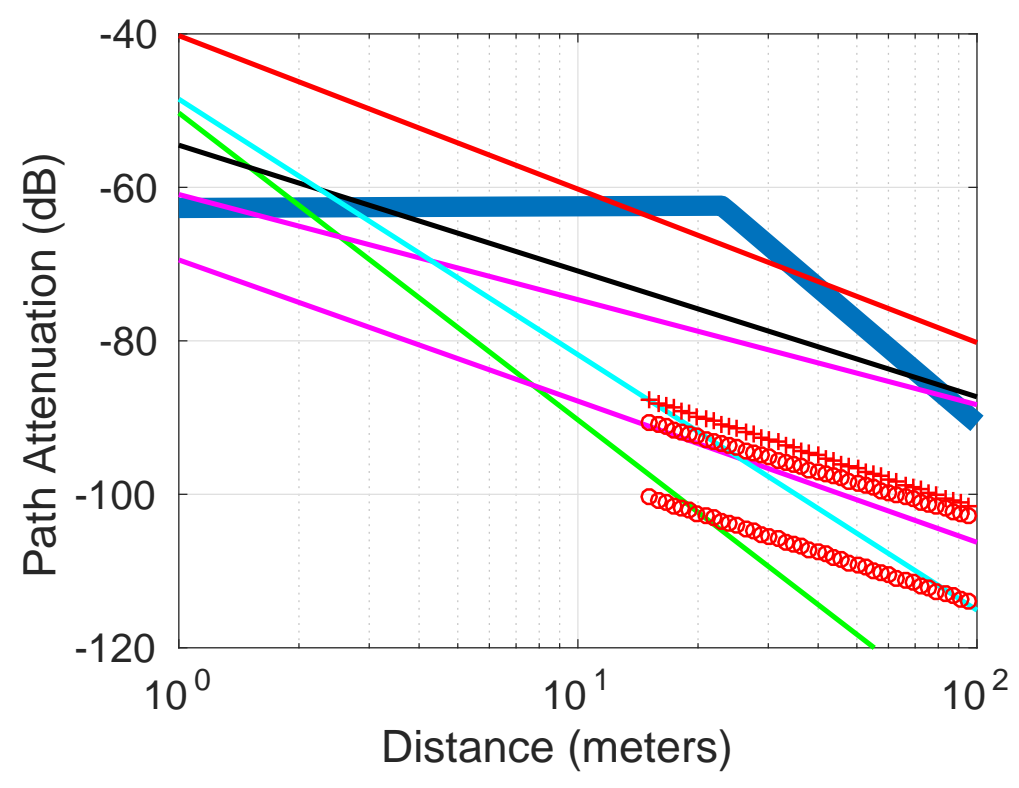

Figure 242: Comparison to other models: Vertical Polarization, 5.4GHz, T1, Inner Path.

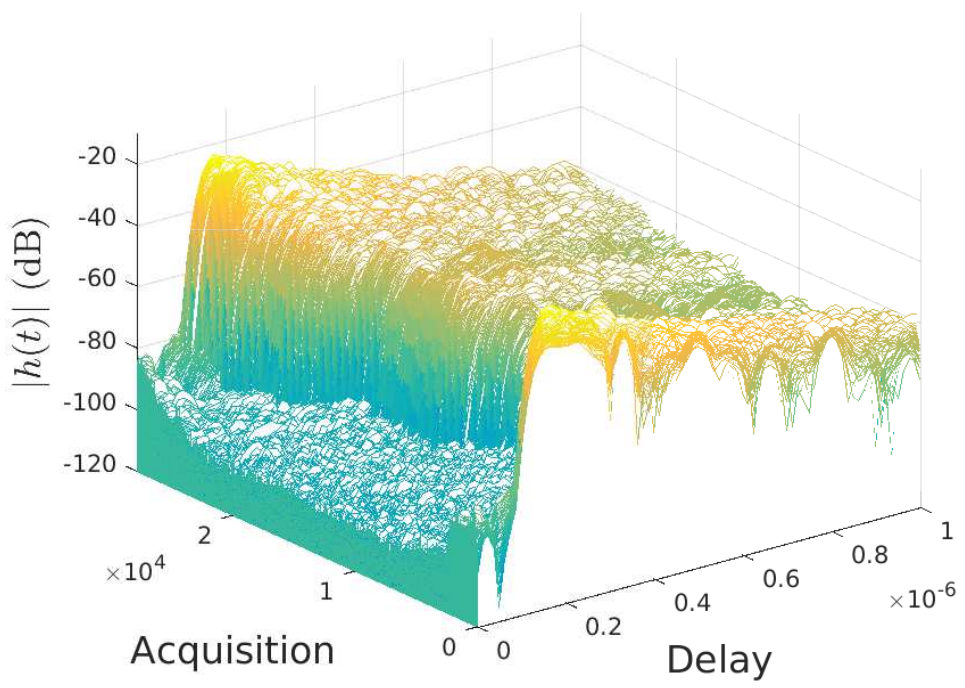

Figure 243: Impulse Responses: Vertical Polarization, 5.4GHz, T1, Inner Path. 


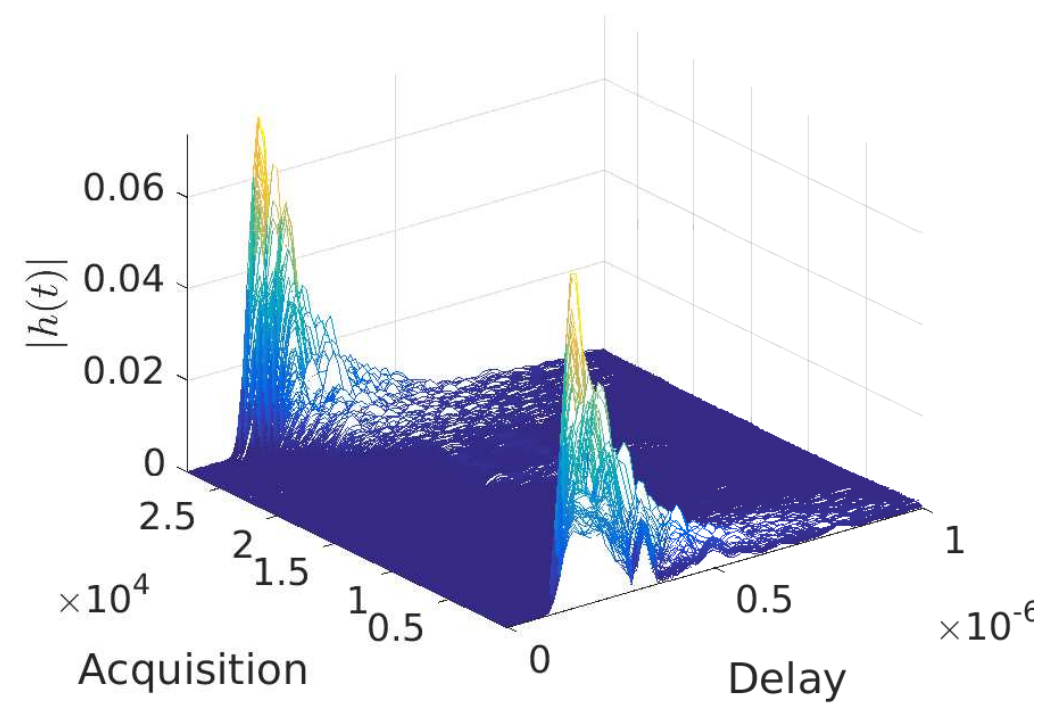

Figure 244: Impulse Responses: Vertical Polarization, 5.4GHz, T1, Inner Path. 


\section{B.13 Vertical Polarization, 2.245GHz, Transmitter 2 Outer Path}

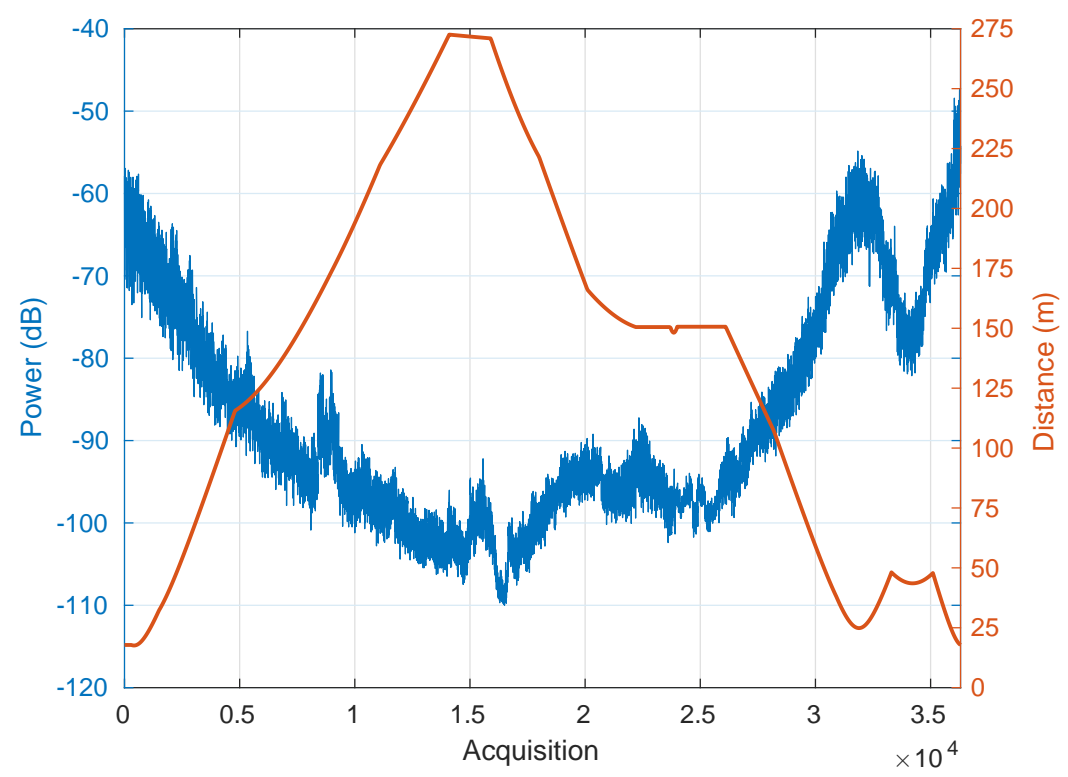

Figure 245: Overall Received Power vs. Acquisition Number and Distance vs. Acquisition Number: Vertical Polarization, 2.245GHz, T2, Outer Path.

\begin{tabular}{c|c|c|c|c|c}
\hline Frequency & Polarization & $\alpha_{1}$ & $\alpha_{2}$ & $\beta(\mathrm{m})$ & $k_{1}(\mathrm{~dB})$ \\
\hline-0.21483 & 4.0867 & 26.3415 & -64.8175 & -3.7079 & 10.6047
\end{tabular}

Table 42: Parameters for Vertical Polarization, 2.245GHz, T2, Outer Path 


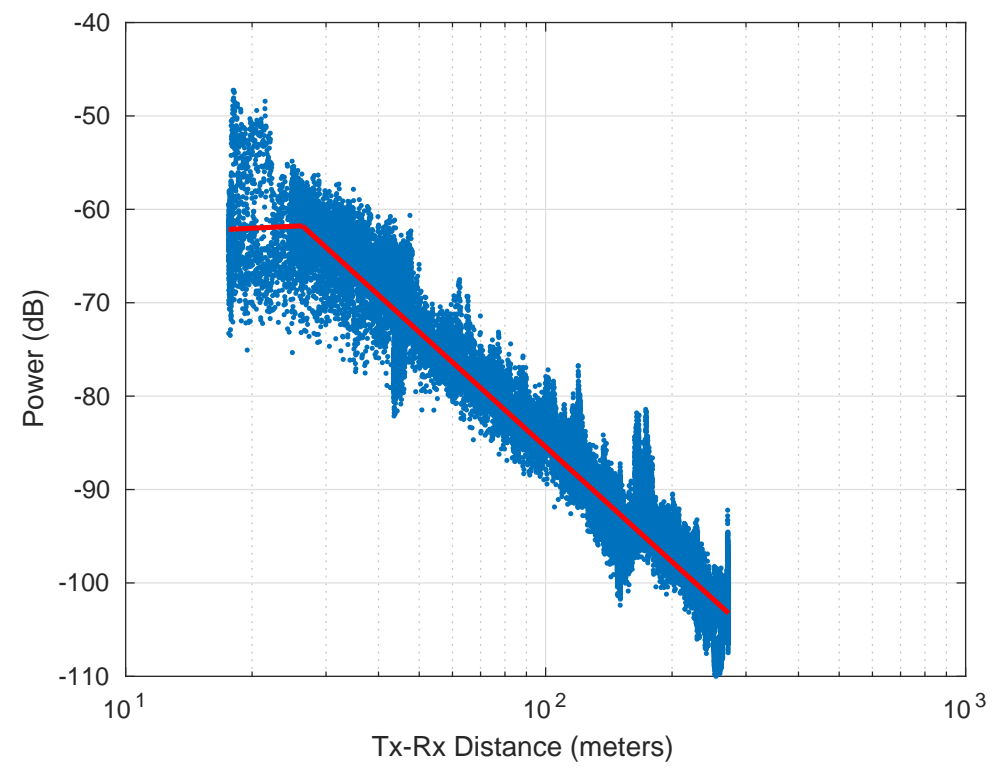

Figure 246: Overall Received Power vs. Distance: Vertical Polarization, 2.245GHz, T2, Outer Path.

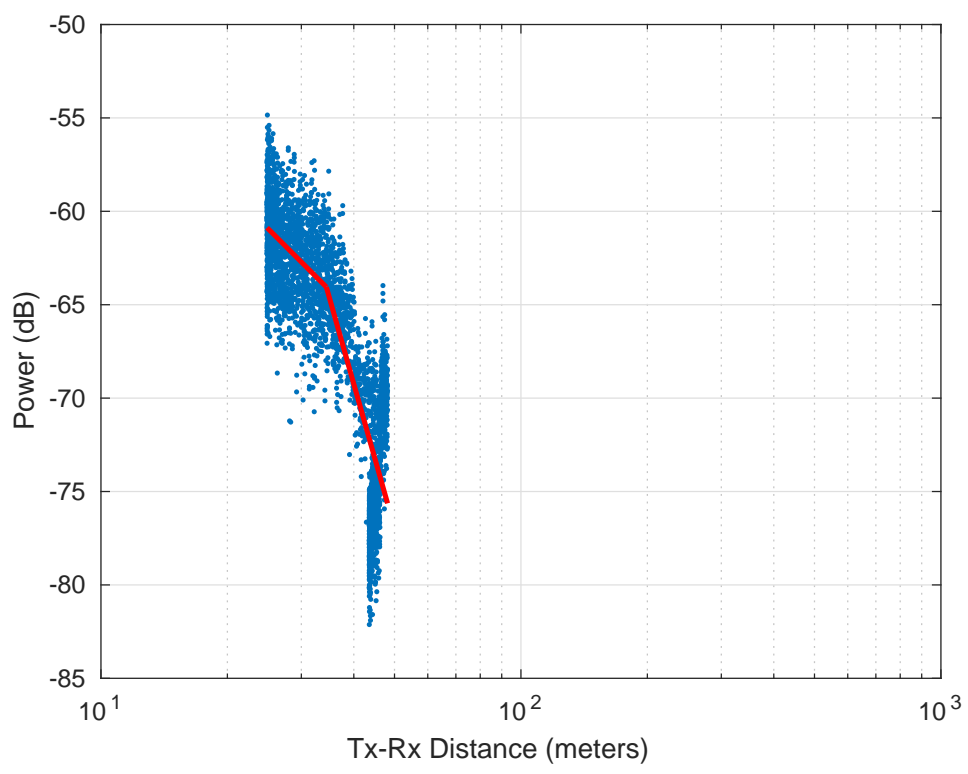

Figure 247: Line of Sight Received Power vs. Distance: Vertical Polarization, 2.245GHz, T2, Outer Path. 


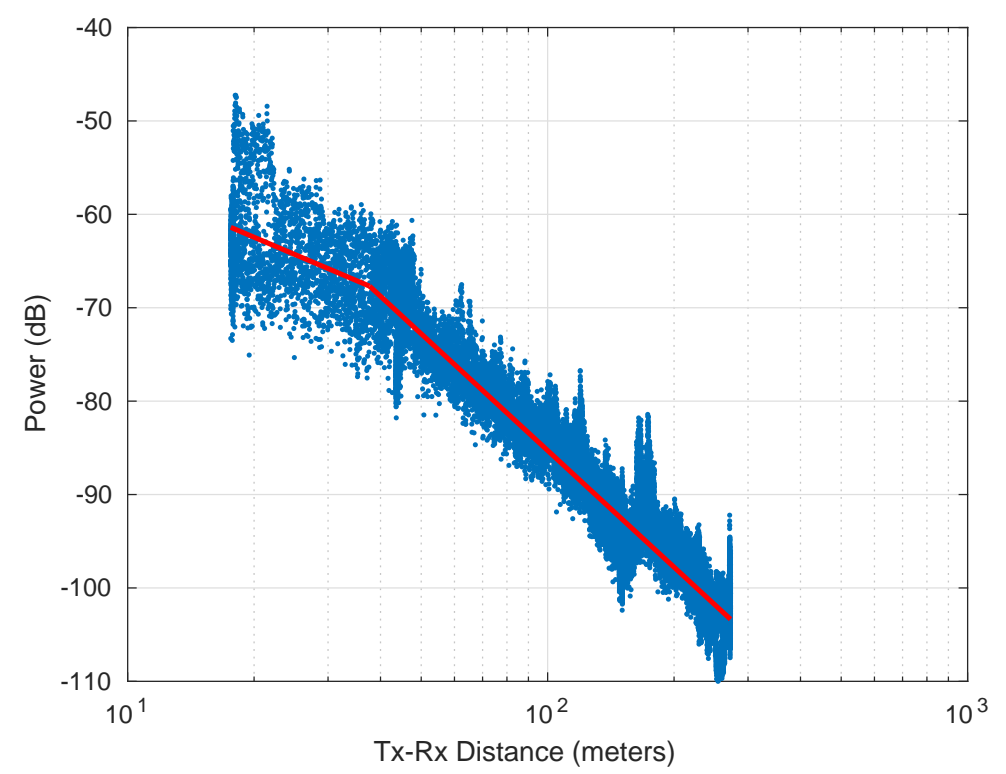

Figure 248: Non Line of Sight Received Power vs. Distance: Vertical Polarization, $2.245 \mathrm{GHz}, \mathrm{T} 2$, Outer Path.

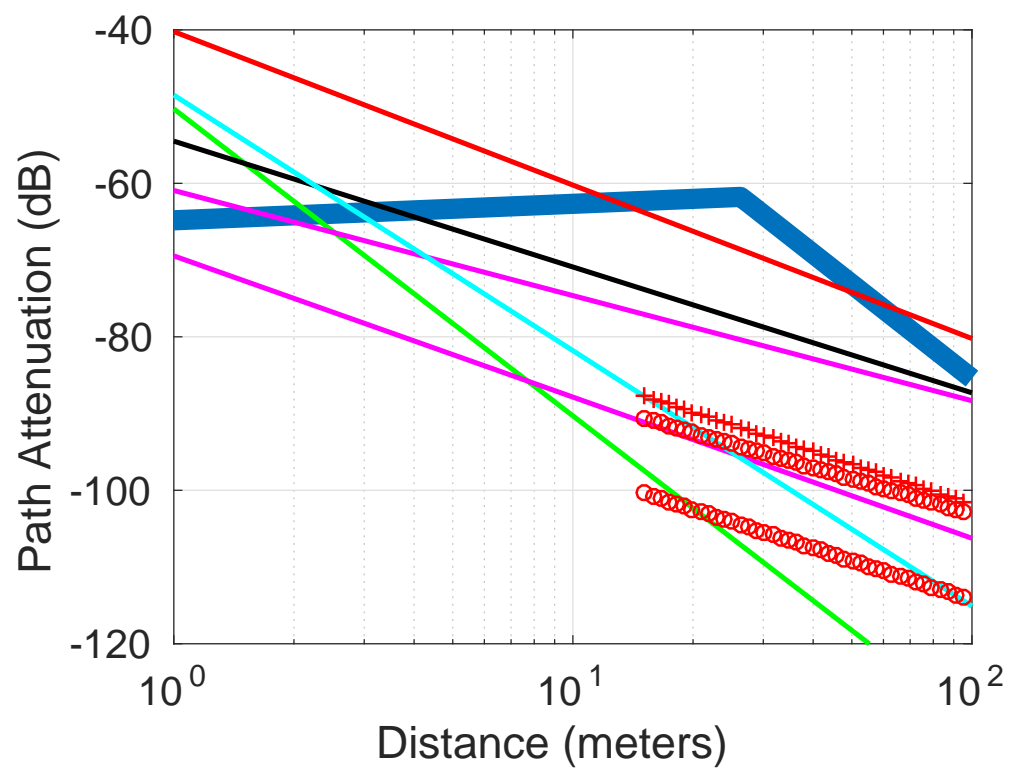

Figure 249: Comparison to other models: Vertical Polarization, 2.245GHz, T2, Outer Path. 


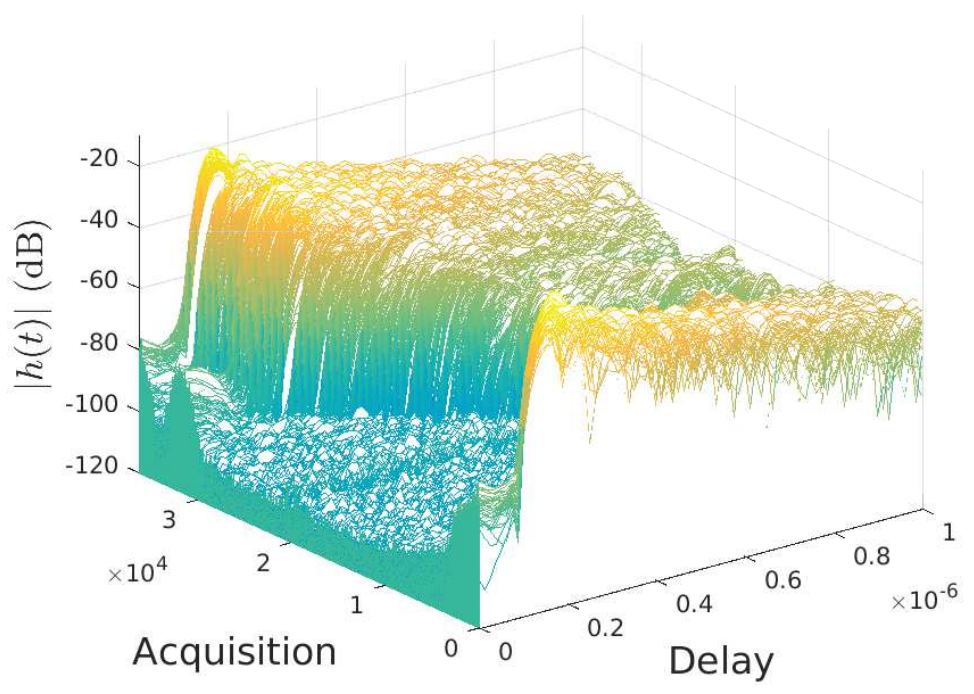

Figure 250: Impulse Responses: Vertical Polarization, 2.245GHz, T2, Outer Path.

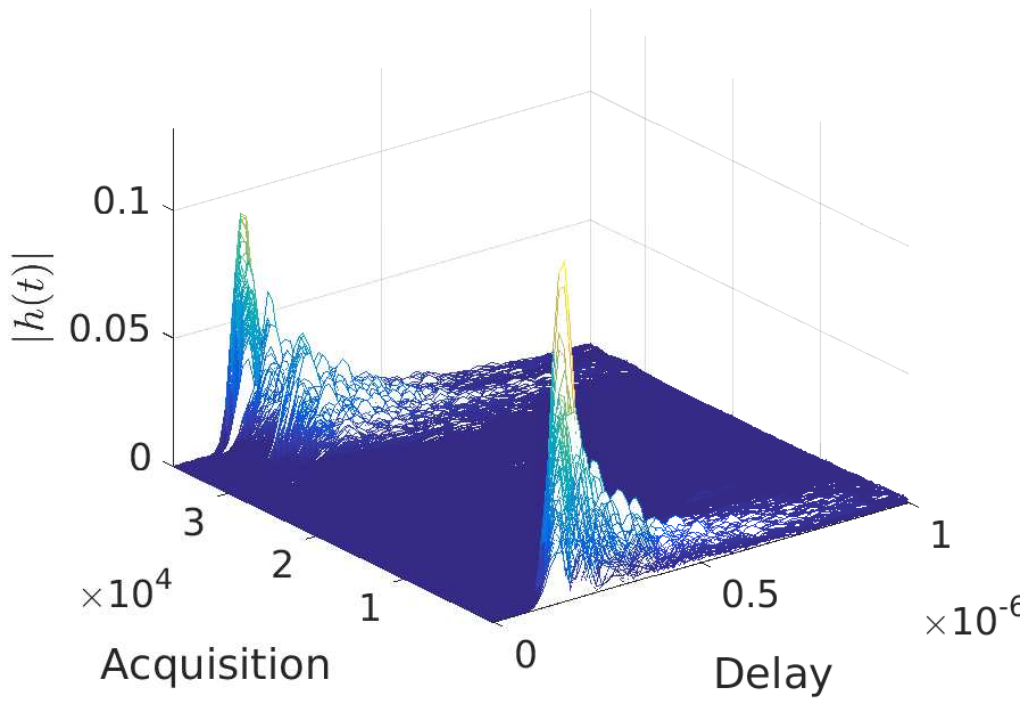

Figure 251: Impulse Responses: Vertical Polarization, 2.245GHz, T2, Outer Path. 


\section{B.14 Vertical Polarization, 5.4GHz, Transmitter 2, Outer Path}

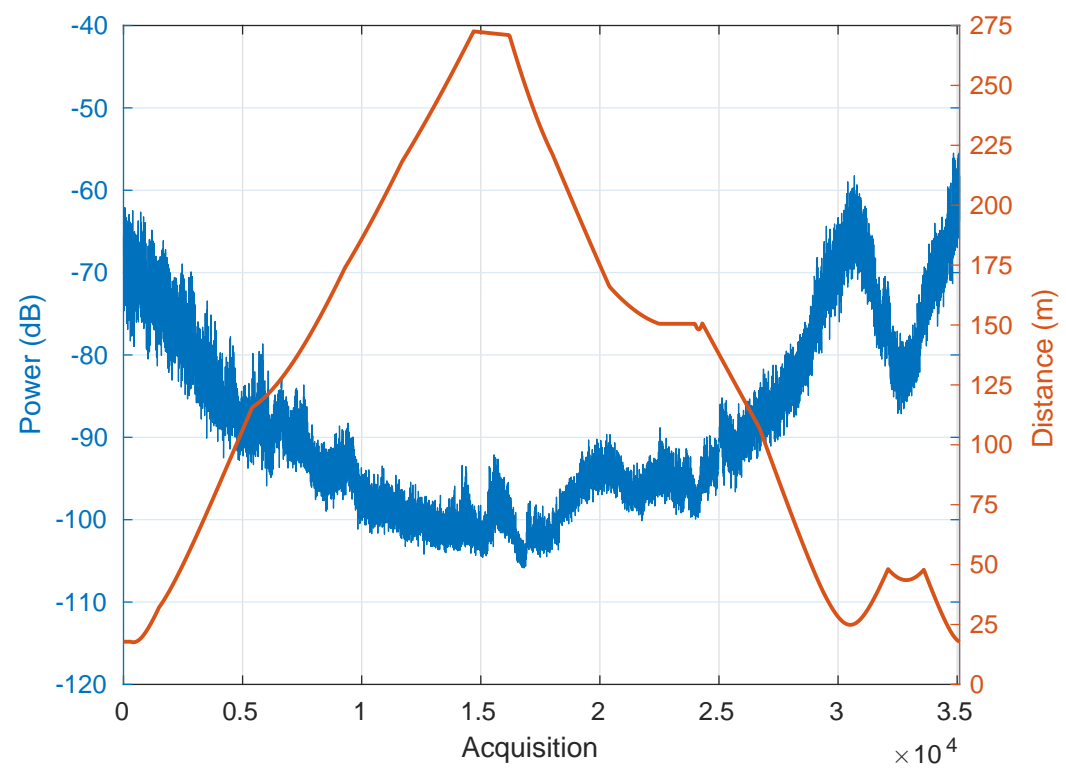

Figure 252: Overall Received Power vs. Acquisition Number and Distance vs. Acquisition Number: Vertical Polarization, 5.4GHz, T2, Outer Path.

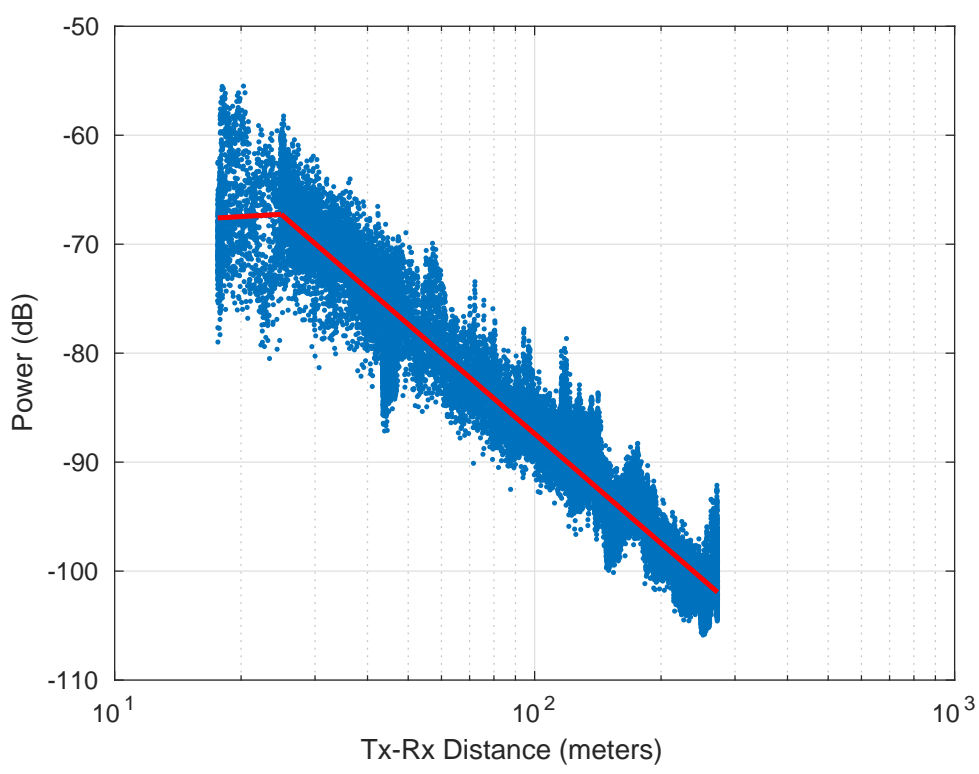

Figure 253: Overall Received Power vs. Distance: Vertical Polarization, 5.4GHz, T2, Outer Path. 


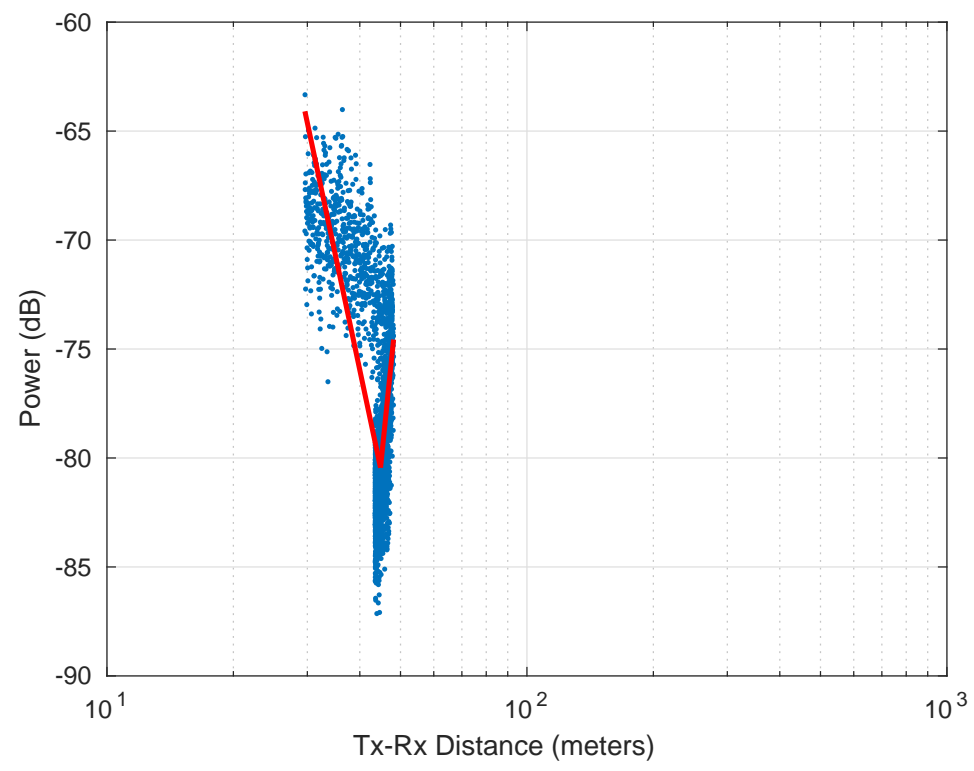

Figure 254: Line of Sight Received Power vs. Distance: Vertical Polarization, 5.4GHz, T2, Outer Path.

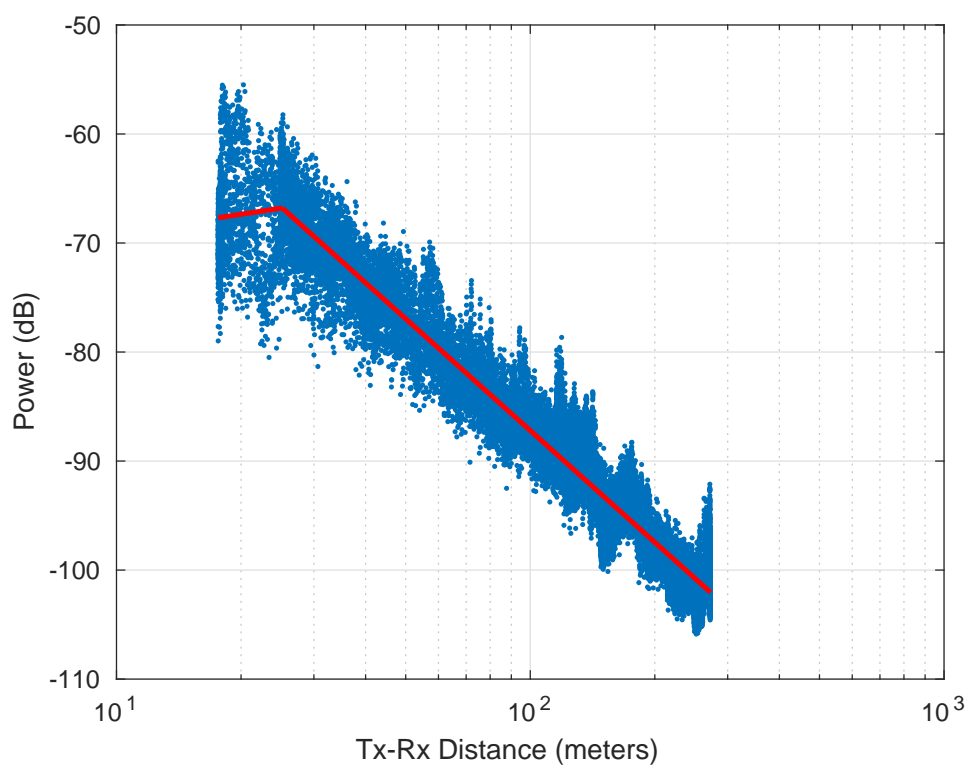

Figure 255: Non Line of Sight Received Power vs. Distance: Vertical Polarization, $5.4 \mathrm{GHz}, \mathrm{T} 2$, Outer Path.

\begin{tabular}{c|c|c|c|c|c}
\hline Frequency & Polarization & $\alpha_{1}$ & $\alpha_{2}$ & $\beta(\mathrm{m})$ & $k_{1}(\mathrm{~dB})$ \\
\hline-0.21652 & 3.3385 & 24.9285 & -70.2831 & -20.6304 & 8.9667
\end{tabular}

Table 43: Parameters for Vertical Polarization, 5.4GHz, T2, Outer Path 


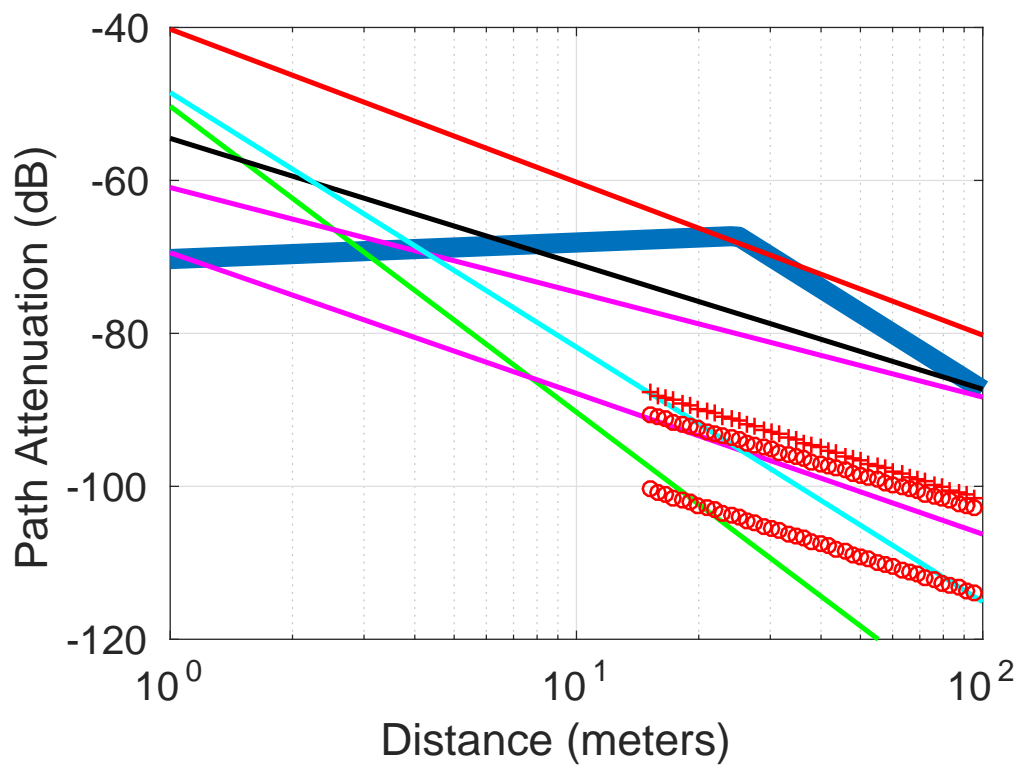

Figure 256: Comparison to other models: Vertical Polarization, 5.4GHz, T2, Outer Path.

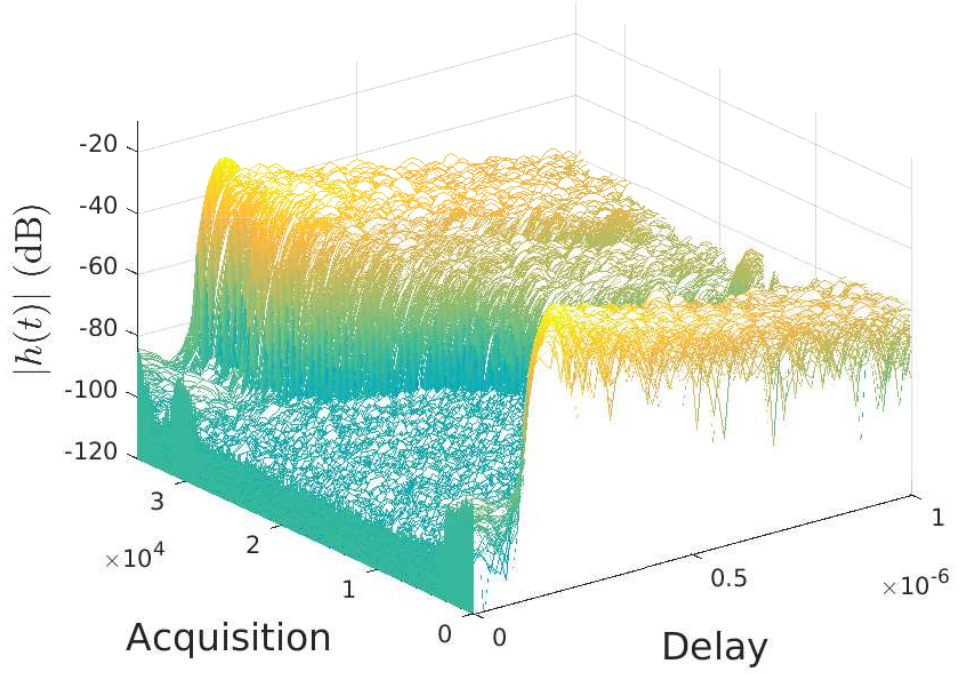

Figure 257: Impulse Responses: Vertical Polarization, 5.4GHz, T2, Outer Path. 


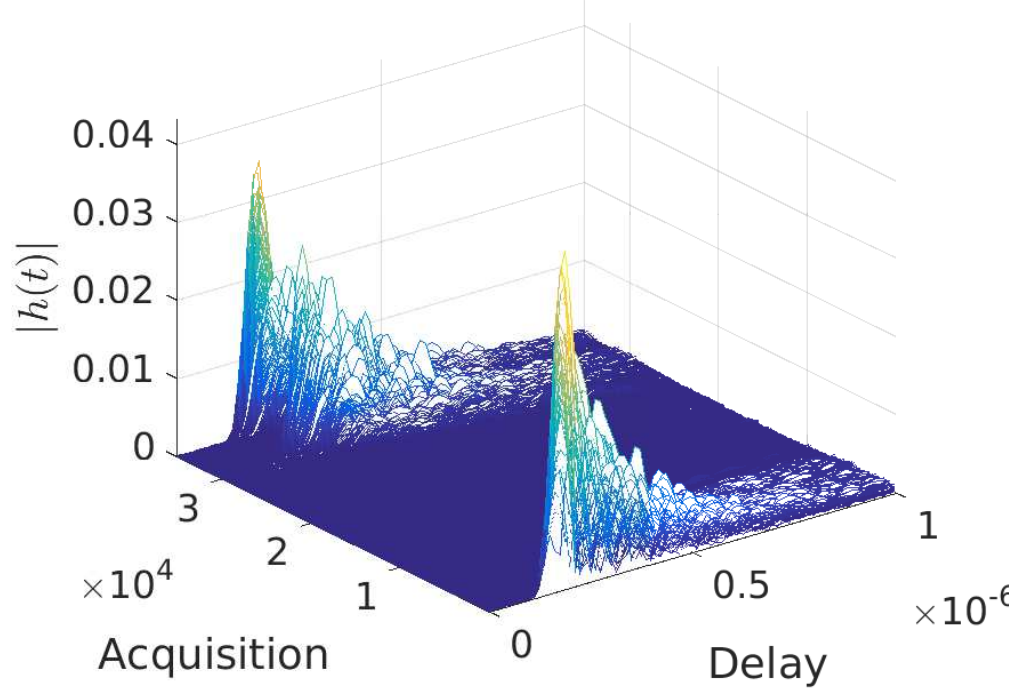

Figure 258: Impulse Responses: Vertical Polarization, 5.4GHz, T2, Outer Path. 


\section{B.15 Vertical Polarization, 2.245GHz, Transmitter 2, Inner Path}

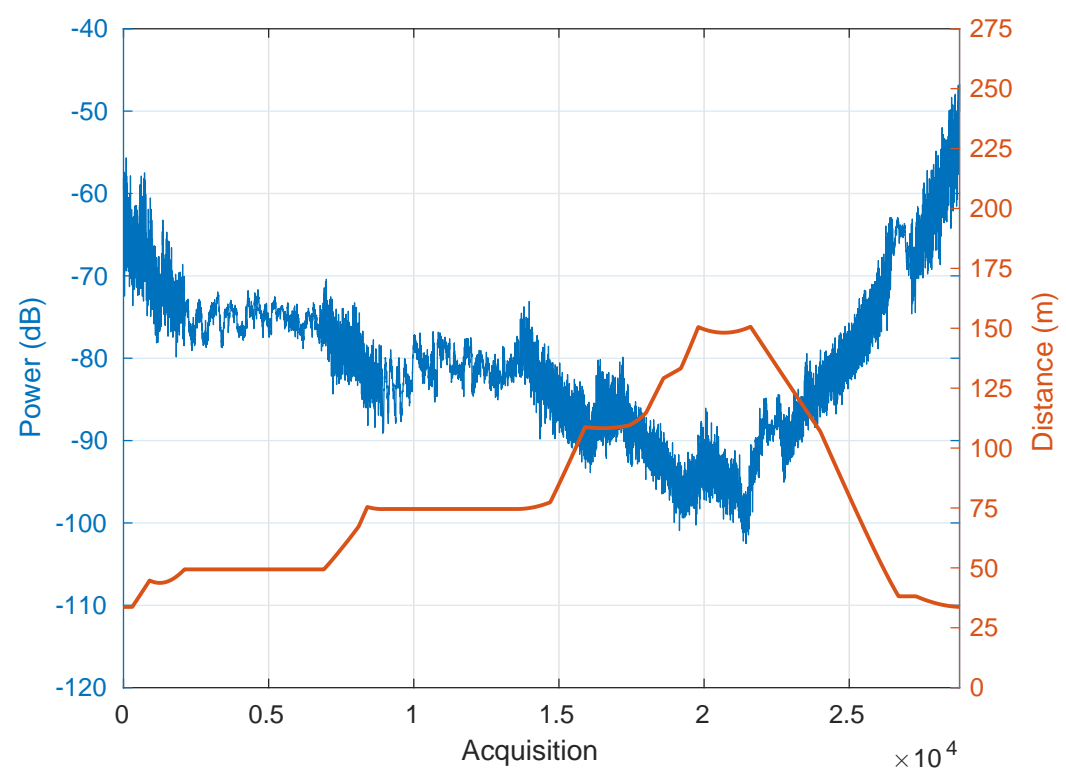

Figure 259: Overall Received Power vs. Acquisition Number and Distance vs. Acquisition Number: Vertical Polarization, 2.245GHz, T2, Inner Path.

\begin{tabular}{c|c|c|c|c|c}
\hline Frequency & Polarization & $\alpha_{1}$ & $\alpha_{2}$ & $\beta(\mathrm{m})$ & $k_{1}(\mathrm{~dB})$ \\
\hline 8.8331 & 3.8846 & 49.3689 & 75.1763 & -8.6227 & 8.9728
\end{tabular}

Table 44: Parameters for Vertical Polarization, 2.245GHz, T2, Inner Path 


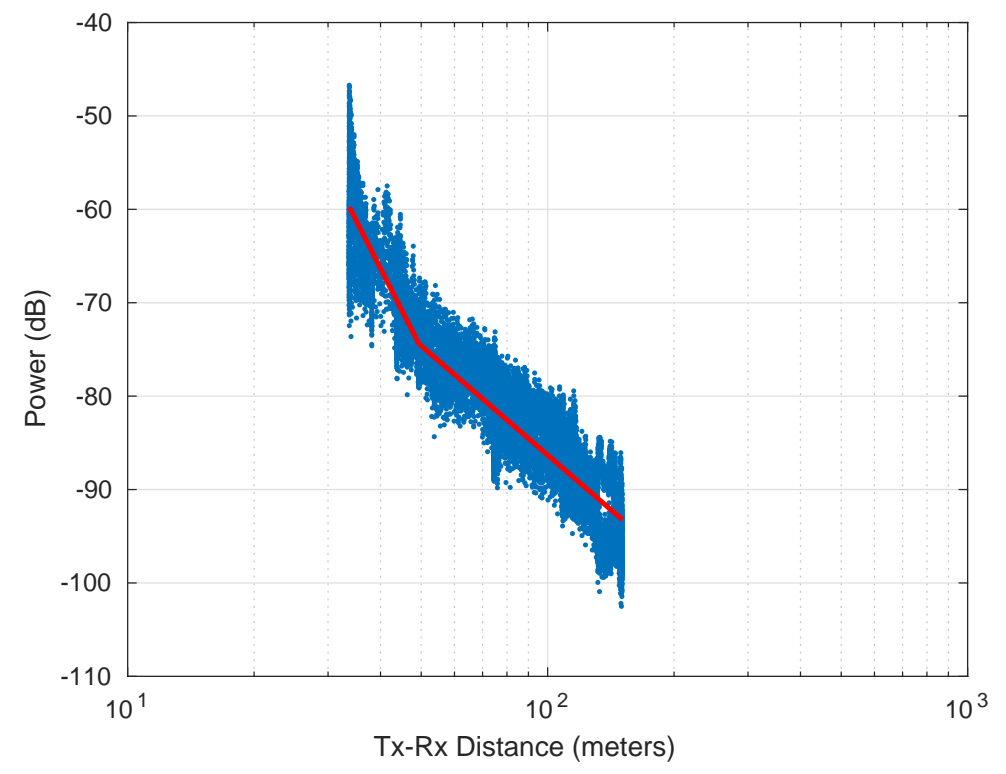

Figure 260: Overall Received Power vs. Distance: Vertical Polarization, 2.245GHz, T2, Inner Path.

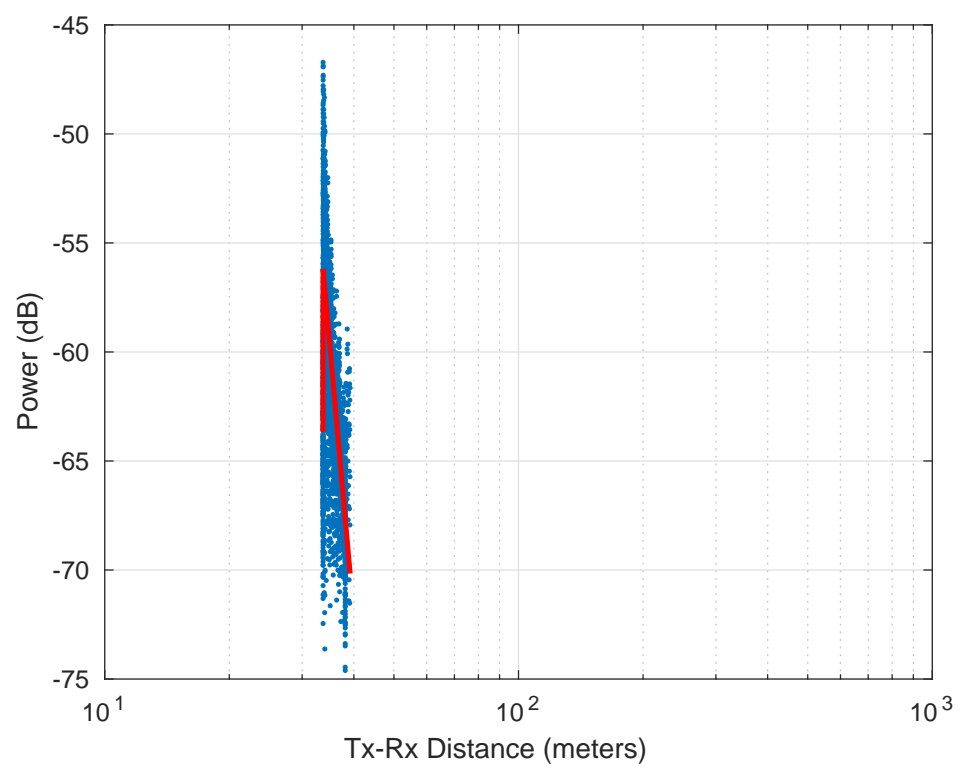

Figure 261: Line of Sight Received Power vs. Distance: Vertical Polarization, 2.245GHz, T2, Inner Path. 


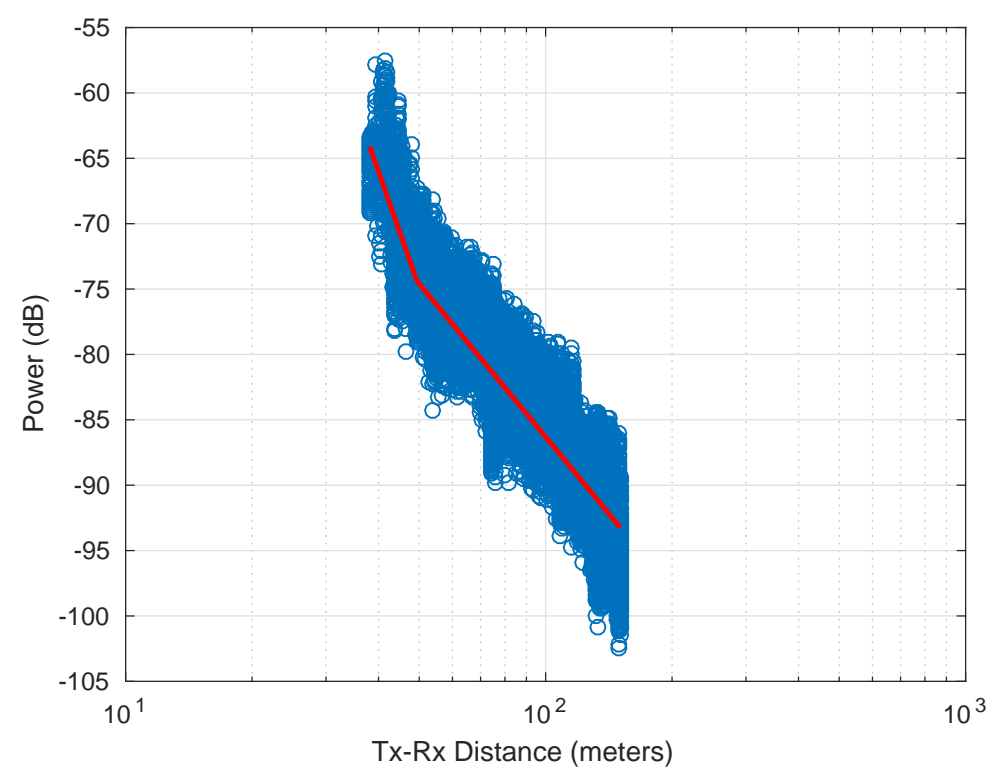

Figure 262: Non Line of Sight Received Power vs. Distance: Vertical Polarization, $2.245 \mathrm{GHz}, \mathrm{T} 2$, Inner Path.

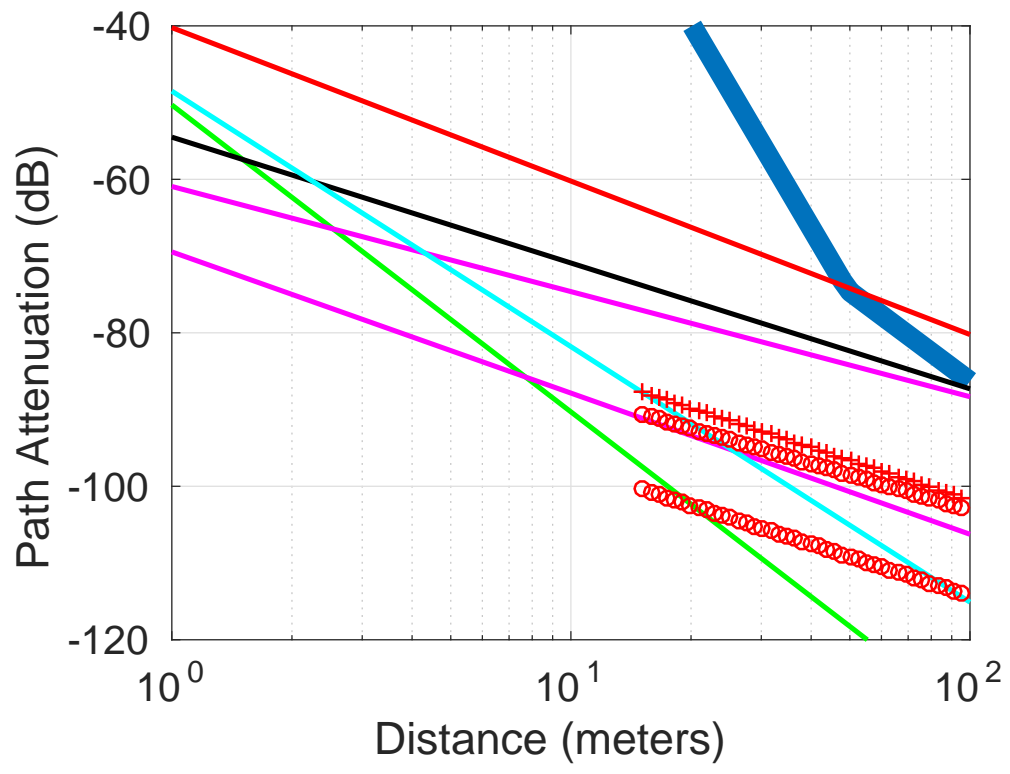

Figure 263: Comparison to other models: Vertical Polarization, 2.245GHz, T2, Inner Path. 


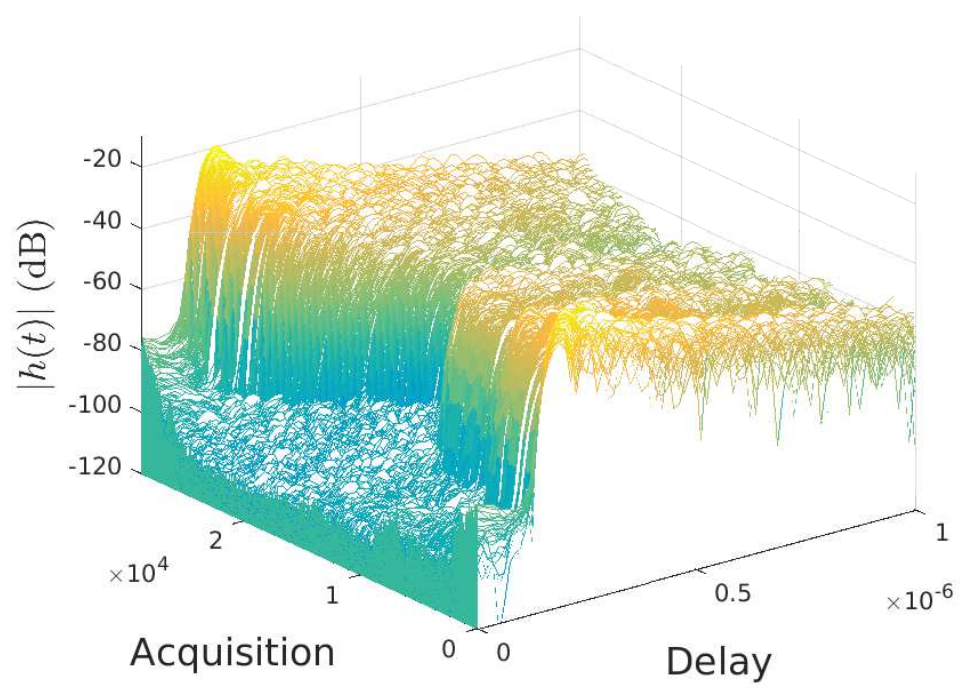

Figure 264: Impulse Responses: Vertical Polarization, 2.245GHz, T2, Inner Path.

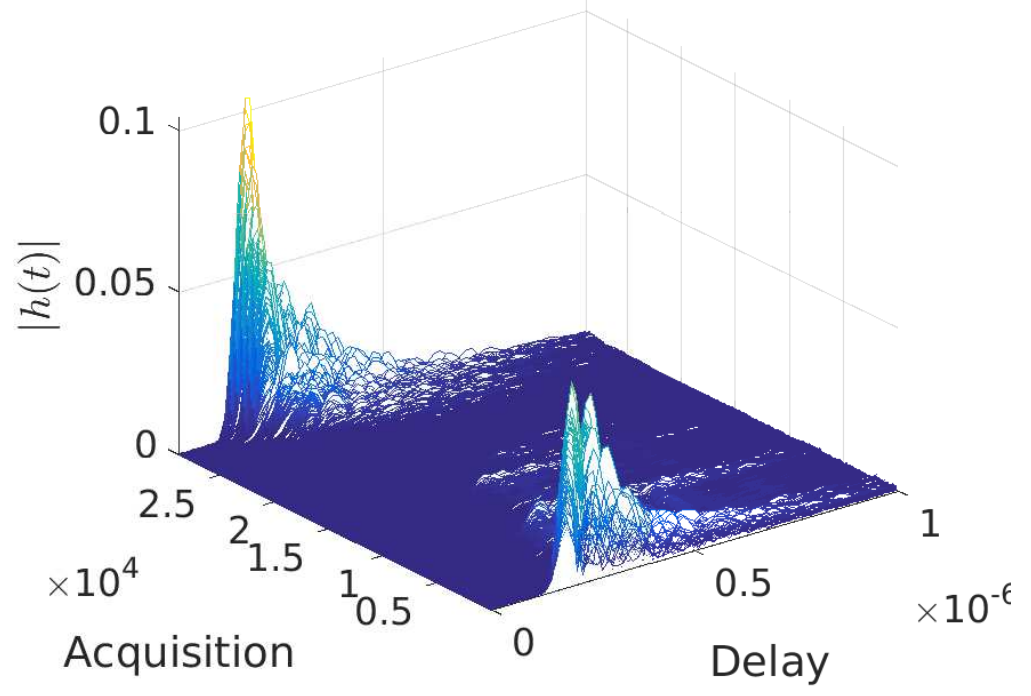

Figure 265: Impulse Responses: Vertical Polarization, 2.245GHz, T2, Inner Path. 


\section{B.16 Vertical Polarization, 5.4GHz, Transmitter 2, Inner Path}

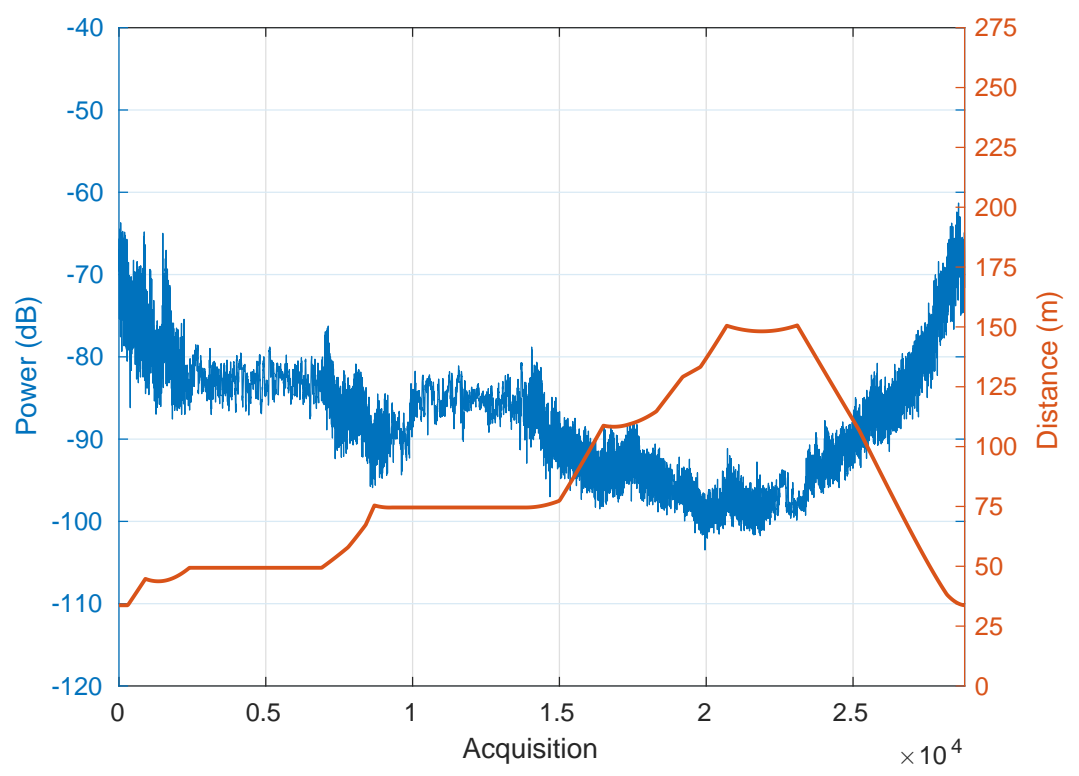

Figure 266: Overall Received Power vs. Acquisition Number and Distance vs. Acquisition Number: Vertical Polarization, 5.4GHz, T2, Inner Path.

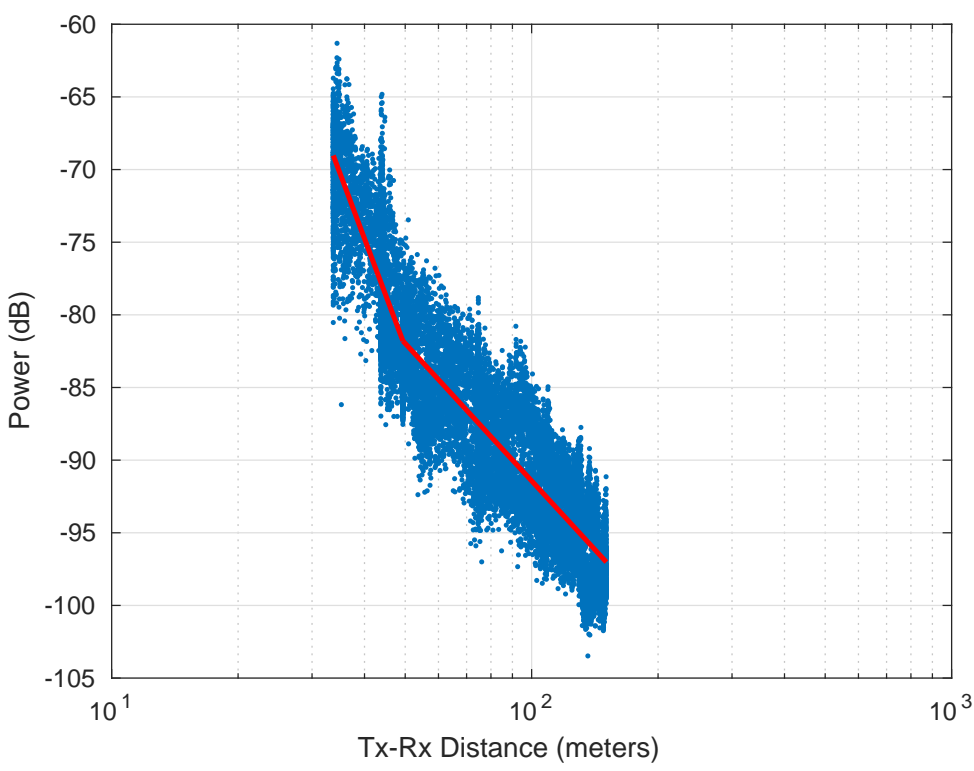

Figure 267: Overall Received Power vs. Distance: Vertical Polarization, 5.4GHz, T2, Inner Path. 


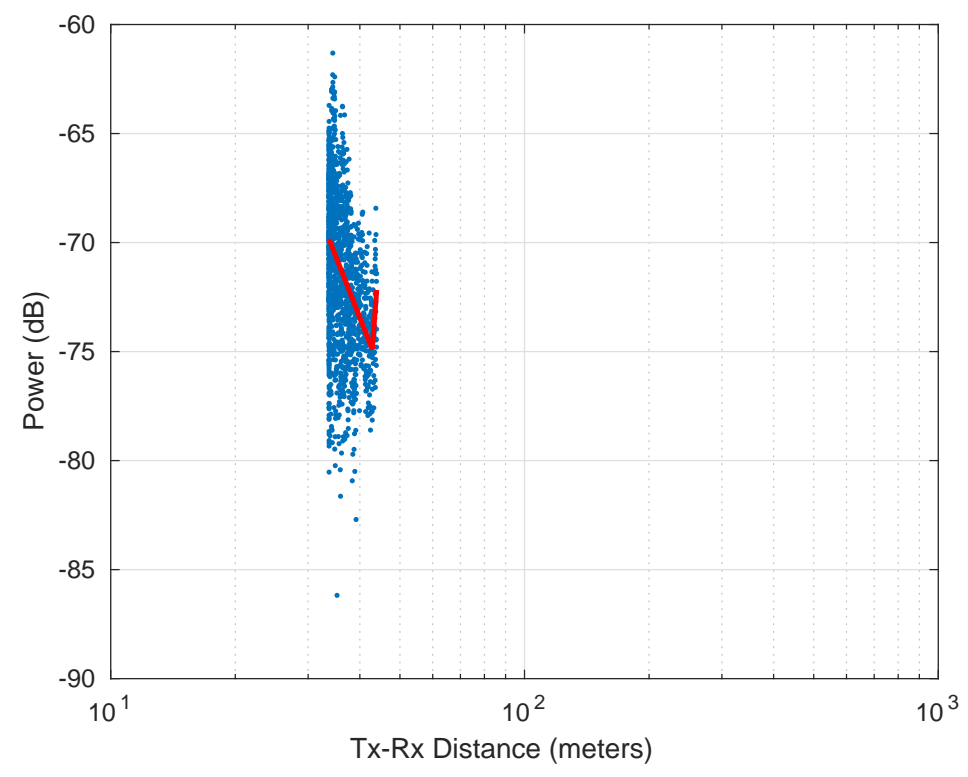

Figure 268: Line of Sight Received Power vs. Distance: Vertical Polarization, 5.4GHz, T2, Inner Path.

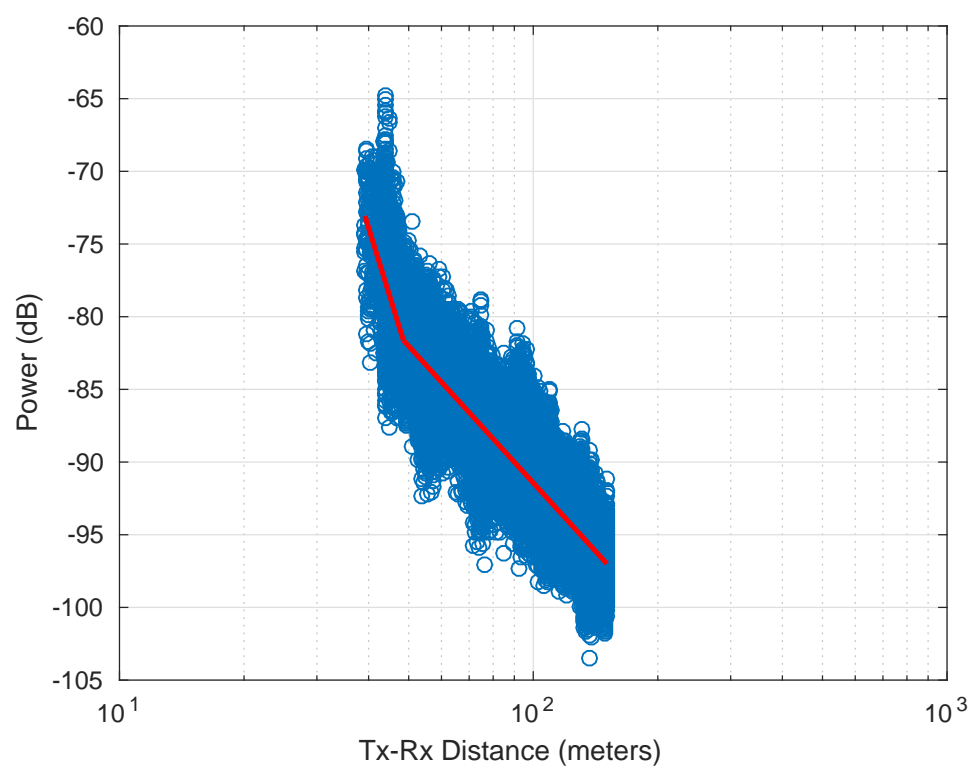

Figure 269: Non Line of Sight Received Power vs. Distance: Vertical Polarization, $5.4 \mathrm{GHz}, \mathrm{T} 2$, Inner Path.

\begin{tabular}{c|c|c|c|c|c}
\hline Frequency & Polarization & $\alpha_{1}$ & $\alpha_{2}$ & $\beta(\mathrm{m})$ & $k_{1}(\mathrm{~dB})$ \\
\hline 7.7008 & 3.1423 & 49.3689 & 48.6145 & -28.5807 & 8.561
\end{tabular}

Table 45: Parameters for Vertical Polarization, 5.4GHz, T2, Inner Path 


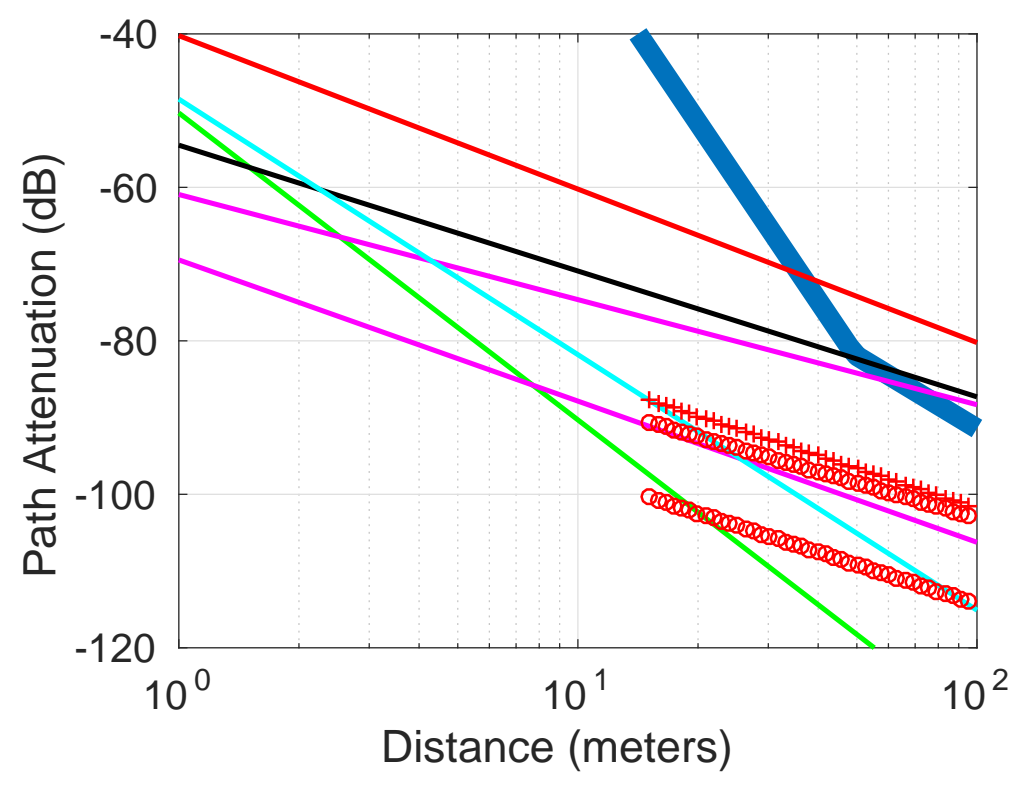

Figure 270: Comparison to other models: Vertical Polarization, 5.4GHz, T2, Inner Path.

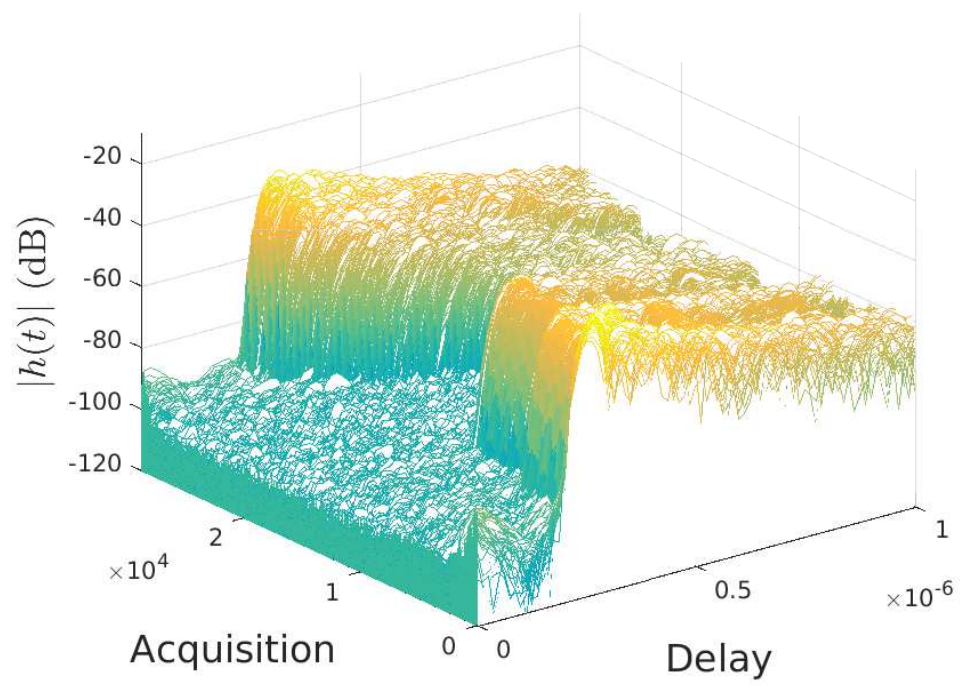

Figure 271: Impulse Responses: Vertical Polarization, 5.4GHz, T2, Inner Path. 


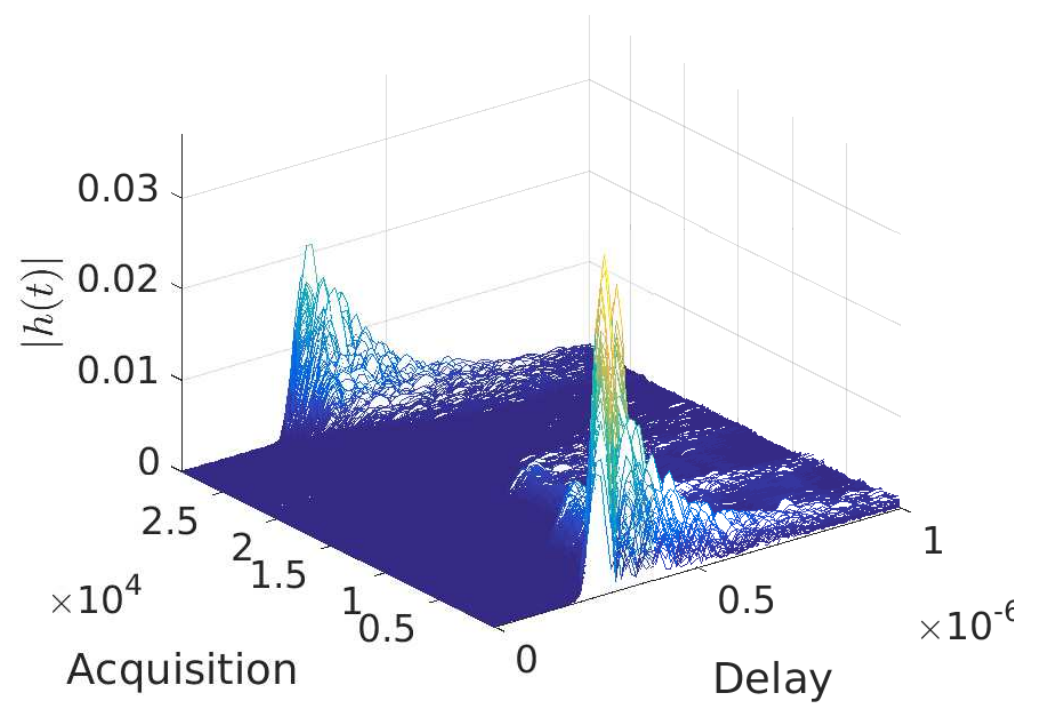

Figure 272: Impulse Responses: Vertical Polarization, 5.4GHz, T2, Inner Path. 


\section{B.17 Channel Parameters Summary}

\begin{tabular}{c|c|c|c|c|c|c|c}
\hline Frequency & Polarization & $\alpha_{1}$ & $\alpha_{2}$ & $\beta(\mathrm{m})$ & $k_{1}(\mathrm{~dB})$ & $k_{2}(\mathrm{~dB})$ & $\mathrm{MSE}$ \\
\hline $2.245 \mathrm{GHz}$ & Vpol & 0.18534 & 4.4378 & 23.4457 & -54.3605 & 3.9013 & 14.9937 \\
$2.245 \mathrm{GHz}$ & Hpol & 0.29658 & 5.1625 & 27.5135 & -57.9935 & 12.054 & 13.0546 \\
$5.4 \mathrm{GHz}$ & Vpol & 0.31471 & 3.8395 & 24.888 & -61.2997 & -12.0941 & 13.7568 \\
$5.4 \mathrm{GHz}$ & Hpol & 0.48352 & 4.1607 & 29.7219 & -66.2907 & -12.1234 & 11.7267
\end{tabular}

Table 46: Transmitter 1 Outer Path: Overall

\begin{tabular}{c|c|c|c|c|c|c|c}
\hline Frequency & Polarization & $\alpha_{1}$ & $\alpha_{2}$ & $\beta(\mathrm{m})$ & $k_{1}(\mathrm{~dB})$ & $k_{2}(\mathrm{~dB})$ & MSE \\
\hline $2.245 \mathrm{GHz}$ & Vpol & 0.16754 & 5.0896 & 23.88 & -56.5334 & 11.2937 & 10.8275 \\
$2.245 \mathrm{GHz}$ & Hpol & 0.5027 & 5.5783 & 32.7876 & -58.3326 & 18.5988 & 11.4318 \\
$5.4 \mathrm{GHz}$ & Vpol & -0.021878 & 4.4378 & 22.9532 & -62.6865 & -1.9973 & 10.3104 \\
$5.4 \mathrm{GHz}$ & Hpol & 0.732 & 5.0265 & 36.55 & -57.8965 & 9.2216 & 10.6267
\end{tabular}

Table 47: Transmitter 1 Inner Path: Overall

\begin{tabular}{c|c|c|c|c|c|c|c}
\hline Frequency & Polarization & $\alpha_{1}$ & $\alpha_{2}$ & $\beta(\mathrm{m})$ & $k_{1}(\mathrm{~dB})$ & $k_{2}(\mathrm{~dB})$ & $\mathrm{MSE}$ \\
\hline $2.245 \mathrm{GHz}$ & Vpol & -0.75769 & 1.4178 & 7.058 & -58.6395 & -40.1762 & 14.8999 \\
$2.245 \mathrm{GHz}$ & Hpol & -0.66414 & 2.0203 & 9.2002 & -62.7633 & -36.8905 & 15.4483 \\
$5.4 \mathrm{GHz}$ & Vpol & -0.70159 & 1.1476 & 5.7494 & -65.6065 & -51.5593 & 13.9725 \\
$5.4 \mathrm{GHz}$ & Hpol & -0.37272 & 1.6244 & 8.8869 & -70.8054 & -51.8574 & 15.6071
\end{tabular}

Table 48: Transmitter 1 Outer Path: Line of Sight

\begin{tabular}{c|c|c|c|c|c|c|c}
\hline Frequency & Polarization & $\alpha_{1}$ & $\alpha_{2}$ & $\beta(\mathrm{m})$ & $k_{1}(\mathrm{~dB})$ & $k_{2}(\mathrm{~dB})$ & MSE \\
\hline $2.245 \mathrm{GHz}$ & Vpol & -0.084129 & 3.394 & 16.6213 & -57.891 & -15.4344 & 14.2126 \\
$2.245 \mathrm{GHz}$ & Hpol & -0.23863 & 3.7294 & 15.0823 & -62.5928 & -15.8306 & 14.1476 \\
$5.4 \mathrm{GHz}$ & Vpol & -0.91379 & 1.7303 & 8.2195 & -66.8826 & -42.6933 & 14.7664 \\
$5.4 \mathrm{GHz}$ & Hpol & 0.22599 & 3.4876 & 18.2132 & -61.0504 & -19.9413 & 12.2895
\end{tabular}

Table 49: Transmitter 1 Inner Path: Line of Sight 


\begin{tabular}{c|c|c|c|c|c|c|c}
\hline Frequency & Polarization & $\alpha_{1}$ & $\alpha_{2}$ & $\beta(\mathrm{m})$ & $k_{1}(\mathrm{~dB})$ & $k_{2}(\mathrm{~dB})$ & $\mathrm{MSE}$ \\
\hline $2.245 \mathrm{GHz}$ & Vpol & 2.8795 & 13.7639 & 157.4082 & -27.7031 & 211.4296 & 13.4936 \\
$2.245 \mathrm{GHz}$ & Hpol & 4.6346 & 8.3827 & 158.6347 & 1.3042 & 83.7783 & 12.0767 \\
$5.4 \mathrm{GHz}$ & Vpol & 2.7112 & 9.6094 & 158.2447 & -35.1829 & 116.5331 & 12.7368 \\
$5.4 \mathrm{GHz}$ & Hpol & 3.6962 & 7.4205 & 160.5689 & -21.586 & 60.5597 & 9.6149
\end{tabular}

Table 50: Transmitter 1 Outer Path: Non Line of Sight

\begin{tabular}{c|c|c|c|c|c|c|c}
\hline Frequency & Polarization & $\alpha_{1}$ & $\alpha_{2}$ & $\beta(\mathrm{m})$ & $k_{1}(\mathrm{~dB})$ & $k_{2}(\mathrm{~dB})$ & $\mathrm{MSE}$ \\
\hline $2.245 \mathrm{GHz}$ & Vpol & 4.3048 & 7.8542 & 89.7508 & -2.4406 & 66.881 & 9.175 \\
$2.245 \mathrm{GHz}$ & Hpol & 4.5526 & 9.8562 & 89.7508 & 1.0973 & 104.6787 & 8.0267 \\
$5.4 \mathrm{GHz}$ & Vpol & 3.5861 & 6.7192 & 86.0828 & -17.0113 & 43.6117 & 7.3207 \\
$5.4 \mathrm{GHz}$ & Hpol & 3.8667 & 8.859 & 89.7508 & -11.3867 & 86.1134 & 8.6872
\end{tabular}

Table 51: Transmitter 1 Inner Path: Non Line of Sight

\begin{tabular}{c|c|c|c|c|c|c|c}
\hline Frequency & Polarization & $\alpha_{1}$ & $\alpha_{2}$ & $\beta(\mathrm{m})$ & $k_{1}(\mathrm{~dB})$ & $k_{2}(\mathrm{~dB})$ & $\mathrm{MSE}$ \\
\hline $2.245 \mathrm{GHz}$ & Vpol & -0.21483 & 4.0867 & 26.3415 & -64.8175 & -3.7079 & 10.6047 \\
$2.245 \mathrm{GHz}$ & Hpol & -1.742 & 4.1473 & 25.3228 & -90.7101 & -8.0524 & 9.6062 \\
$5.4 \mathrm{GHz}$ & Vpol & -0.21652 & 3.3385 & 24.9285 & -70.2831 & -20.6304 & 8.9667 \\
$5.4 \mathrm{GHz}$ & Hpol & 3.3884 & 2.0603 & 143.51 & -27.2338 & -55.878 & 7.5173
\end{tabular}

Table 52: Transmitter 2 Outer Path: Overall

\begin{tabular}{c|c|c|c|c|c|c|c}
\hline Frequency & Polarization & $\alpha_{1}$ & $\alpha_{2}$ & $\beta(\mathrm{m})$ & $k_{1}(\mathrm{~dB})$ & $k_{2}(\mathrm{~dB})$ & $\mathrm{MSE}$ \\
\hline $2.245 \mathrm{GHz}$ & Vpol & 8.8331 & 3.8846 & 49.3689 & 75.1763 & -8.6227 & 8.9728 \\
$2.245 \mathrm{GHz}$ & Hpol & 3.2852 & 4.7235 & 74.5645 & -23.9979 & 2.9344 & 9.1289 \\
$5.4 \mathrm{GHz}$ & Vpol & 7.7008 & 3.1423 & 49.3689 & 48.6145 & -28.5807 & 8.561 \\
$5.4 \mathrm{GHz}$ & Hpol & 15.8421 & 3.4173 & 40.3601 & 172.8361 & -26.7001 & 6.6621
\end{tabular}

Table 53: Transmitter 2 Inner Path: Overall

\begin{tabular}{c|c|c|c|c|c|c|c}
\hline Frequency & Polarization & $\alpha_{1}$ & $\alpha_{2}$ & $\beta(\mathrm{m})$ & $k_{1}(\mathrm{~dB})$ & $k_{2}(\mathrm{~dB})$ & MSE \\
\hline $2.245 \mathrm{GHz}$ & Vpol & 2.2504 & 7.9462 & 34.4113 & -29.4719 & 58.0555 & 10.1098 \\
$2.245 \mathrm{GHz}$ & Hpol & 8.8961 & -11.1197 & 43.8763 & 68.2184 & -260.4875 & 11.2724 \\
$5.4 \mathrm{GHz}$ & Vpol & 9.0811 & -18.9328 & 44.8101 & 69.5184 & -393.0975 & 10.8363 \\
$5.4 \mathrm{GHz}$ & Hpol & 7.2839 & -18.2791 & 44.9249 & 35.0228 & -387.4029 & 8.3679
\end{tabular}

Table 54: Transmitter 2 Outer Path: Line of Sight 


\begin{tabular}{c|c|c|c|c|c|c|c}
\hline Frequency & Polarization & $\alpha_{1}$ & $\alpha_{2}$ & $\beta(\mathrm{m})$ & $k_{1}(\mathrm{~dB})$ & $k_{2}(\mathrm{~dB})$ & $\mathrm{MSE}$ \\
\hline $2.245 \mathrm{GHz}$ & Vpol & -132352.2889 & 21.3922 & 33.705 & -2021995.2006 & 270.6132 & 10.7049 \\
$2.245 \mathrm{GHz}$ & Hpol & -38040.7894 & 4.5696 & 33.7057 & -581219.2318 & 0.99317 & 11.3102 \\
$5.4 \mathrm{GHz}$ & Vpol & 4.8438 & -25.471 & 42.8573 & 4.153 & -490.5918 & 10.2566 \\
$5.4 \mathrm{GHz}$ & Hpol & 18312.6092 & 12.5486 & 33.7057 & 279691.9431 & 121.3031 & 9.4726
\end{tabular}

Table 55: Transmitter 2 Inner Path: Line of Sight

\begin{tabular}{c|c|c|c|c|c|c|c}
\hline Frequency & Polarization & $\alpha_{1}$ & $\alpha_{2}$ & $\beta(\mathrm{m})$ & $k_{1}(\mathrm{~dB})$ & $k_{2}(\mathrm{~dB})$ & $\mathrm{MSE}$ \\
\hline $2.245 \mathrm{GHz}$ & Vpol & 1.9042 & 4.1499 & 37.6736 & -37.6778 & -2.2841 & 10.5129 \\
$2.245 \mathrm{GHz}$ & Hpol & -1.6507 & 4.125 & 25.2495 & -89.5485 & -8.5582 & 9.3197 \\
$5.4 \mathrm{GHz}$ & Vpol & -0.57855 & 3.4058 & 25.1524 & -74.8902 & -19.0862 & 8.3108 \\
$5.4 \mathrm{GHz}$ & Hpol & 3.3824 & 2.0643 & 143.8879 & -27.3387 & -55.7838 & 7.2467
\end{tabular}

Table 56: Transmitter 2 Outer Path: Non Line of Sight

\begin{tabular}{c|c|c|c|c|c|c|c}
\hline Frequency & Polarization & $\alpha_{1}$ & $\alpha_{2}$ & $\beta(\mathrm{m})$ & $k_{1}(\mathrm{~dB})$ & $k_{2}(\mathrm{~dB})$ & $\mathrm{MSE}$ \\
\hline $2.245 \mathrm{GHz}$ & Vpol & 9.1563 & 3.8934 & 49.3689 & 80.6796 & -8.444 & 8.3805 \\
$2.245 \mathrm{GHz}$ & Hpol & 2.3566 & 4.9158 & 74.5645 & -40.9341 & 6.988 & 7.9821 \\
$5.4 \mathrm{GHz}$ & Vpol & 9.2104 & 3.1256 & 48.5337 & 73.6704 & -28.9225 & 8.3969 \\
$5.4 \mathrm{GHz}$ & Hpol & 1.928 & 4.1254 & 67.2739 & -52.4452 & -12.2803 & 5.3281
\end{tabular}

Table 57: Transmitter 2 Inner Path: Non Line of Sight 


\begin{tabular}{c|c|c|c|c}
\hline Frequency & TX & Polarization & RMS Delay Spread & K-factor \\
\hline $2.245 \mathrm{GHz}$ & 1 & Vpol & $4.0592 \mathrm{e}-06$ & 0.49331 \\
$2.245 \mathrm{GHz}$ & 1 & Hpol & $3.6064 \mathrm{e}-06$ & 0.25236 \\
$5.4 \mathrm{GHz}$ & 1 & Vpol & $3.5683 \mathrm{e}-06$ & 0.40522 \\
$5.4 \mathrm{GHz}$ & 1 & Hpol & $5.2778 \mathrm{e}-06$ & 0.2813 \\
$2.245 \mathrm{GHz}$ & 1 & Vpol & $1.7529 \mathrm{e}-06$ & 0.42281 \\
$2.245 \mathrm{GHz}$ & 1 & Hpol & $2.4429 \mathrm{e}-06$ & 0.30233 \\
$5.4 \mathrm{GHz}$ & 1 & Vpol & $4.3865 \mathrm{e}-06$ & 0.3753 \\
$5.4 \mathrm{GHz}$ & 1 & Hpol & $2.1796 \mathrm{e}-06$ & 0.23678 \\
$2.245 \mathrm{GHz}$ & 2 & Vpol & $2.921 \mathrm{e}-06$ & 0.38664 \\
$2.245 \mathrm{GHz}$ & 2 & Hpol & $4.2243 \mathrm{e}-06$ & 0.28514 \\
$5.4 \mathrm{GHz}$ & 2 & Vpol & $2.8158 \mathrm{e}-06$ & 0.38122 \\
$5.4 \mathrm{GHz}$ & 2 & Hpol & $5.8357 \mathrm{e}-06$ & 0.22054 \\
$2.245 \mathrm{GHz}$ & 2 & Vpol & $1.3924 \mathrm{e}-06$ & 0.30872 \\
$2.245 \mathrm{GHz}$ & 2 & Hpol & $3.3151 \mathrm{e}-06$ & 0.31225 \\
$5.4 \mathrm{GHz}$ & 2 & Vpol & $2.1208 \mathrm{e}-06$ & 0.28093 \\
$5.4 \mathrm{GHz}$ & 2 & Hpol & $3.3483 \mathrm{e}-06$ & 0.22346
\end{tabular}




\section{B.18 2.245GHz, Transmitter 1}

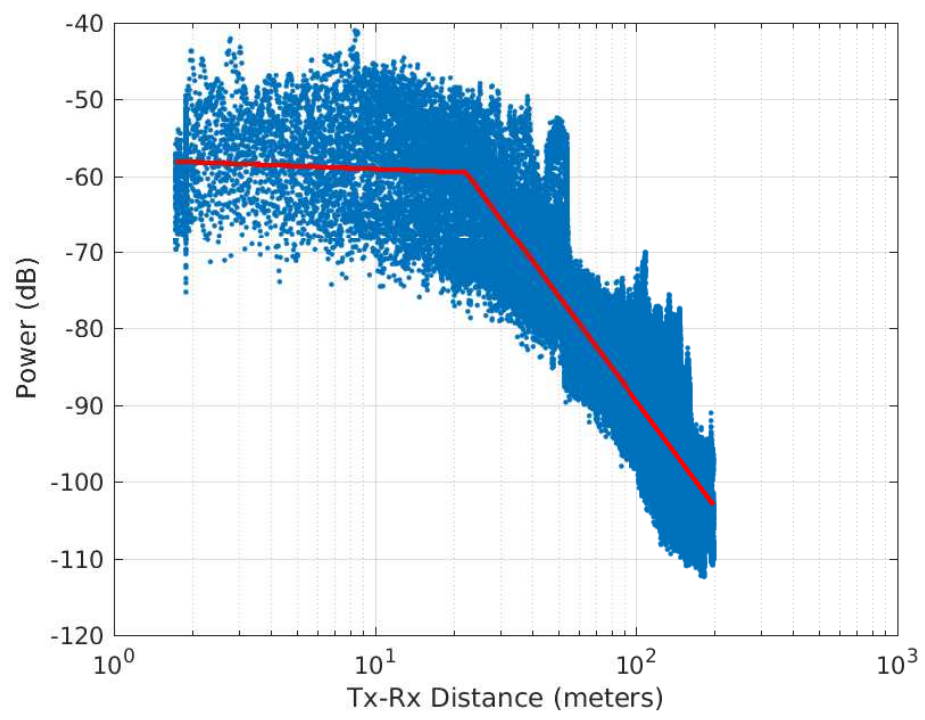

Figure 273: Overall Received Power vs. Distance: All Polarizations, 2.245GHz, T1.

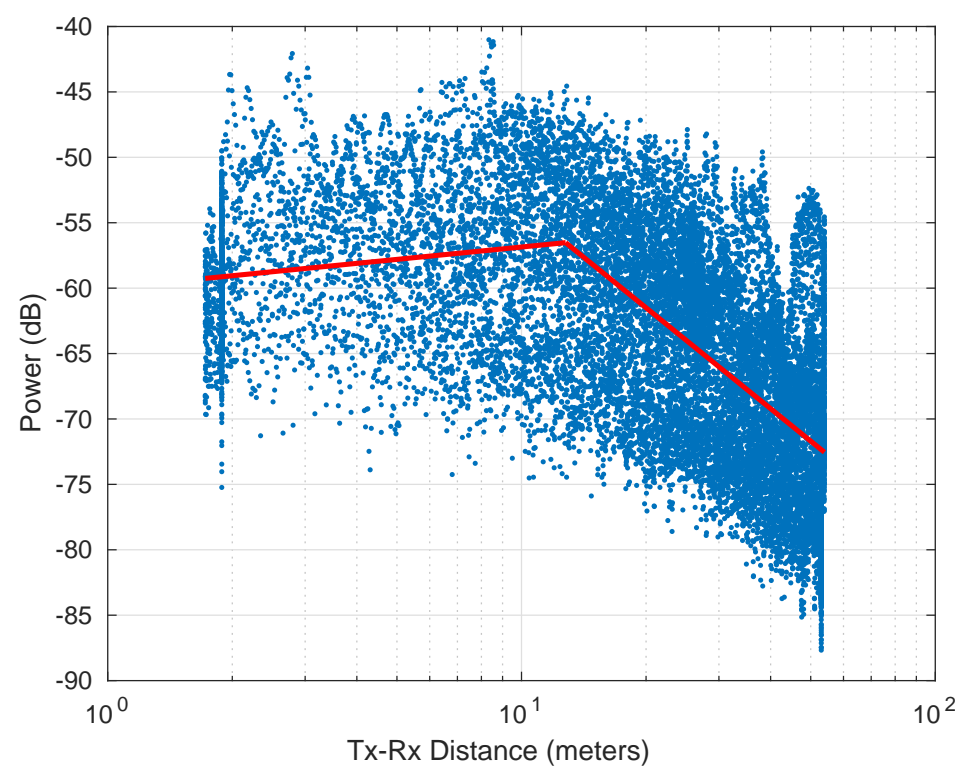

Figure 274: Line of Sight Received Power vs. Distance: All Polarizations, 2.245GHz, T1. 


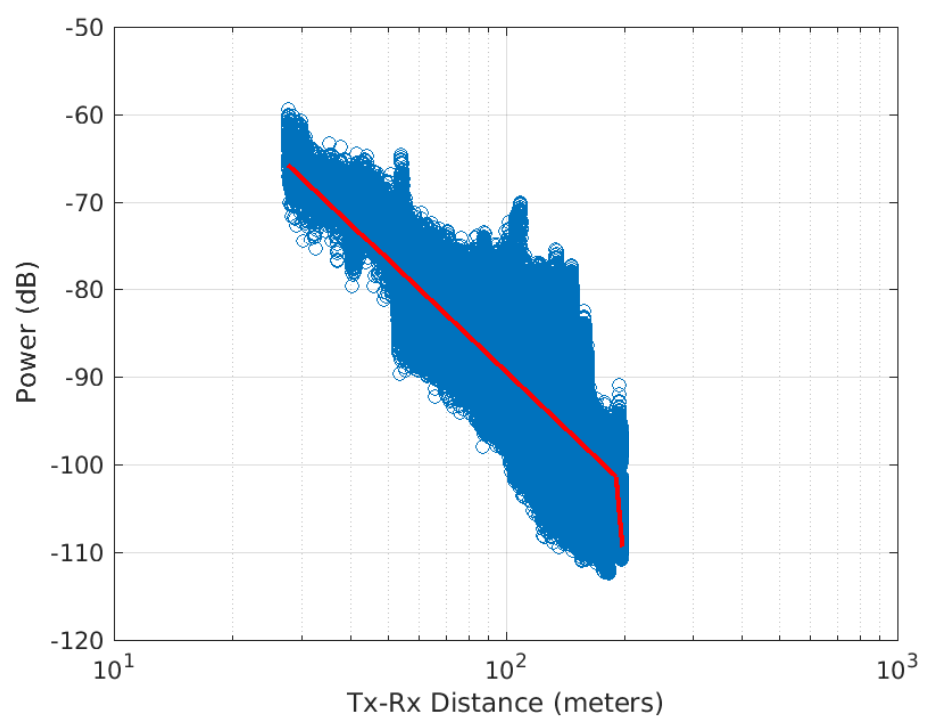

Figure 275: Non Line of Sight Received Power vs. Distance: All Polarizations, 2.245GHz, $\mathrm{T} 1$.

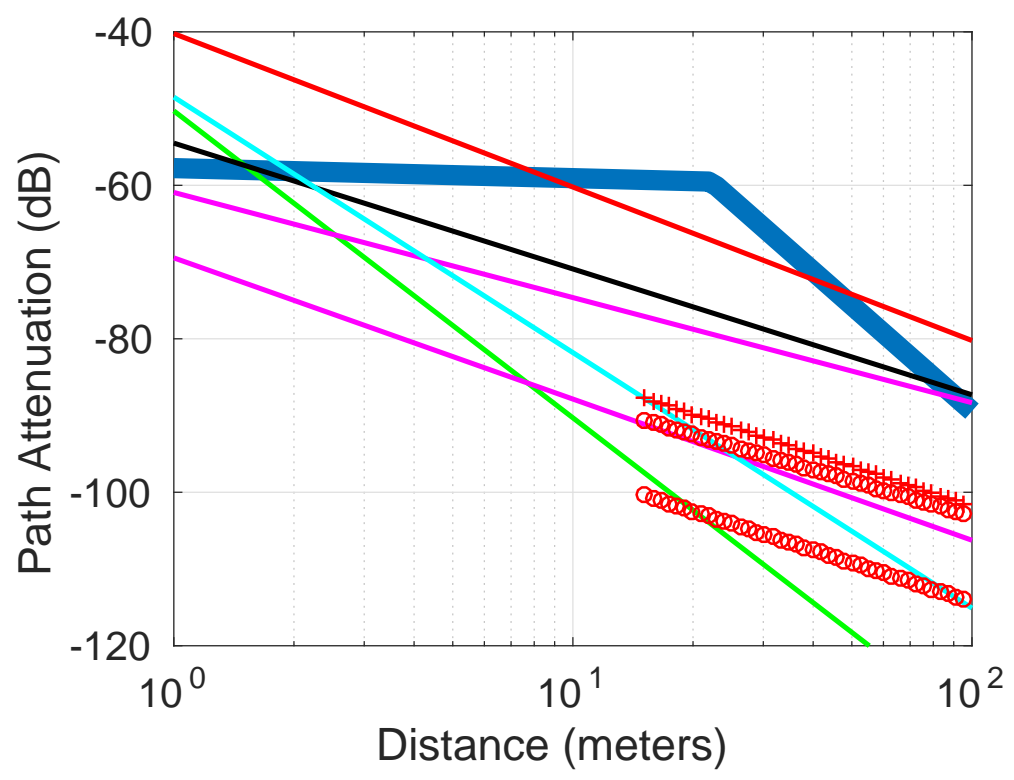

Figure 276: Comparison to other models: All Polarizations, 2.245GHz, T1.

\begin{tabular}{c|c|c|c|c|c}
\hline$\alpha_{1}$ & $\alpha_{2}$ & $\beta(\mathrm{m})$ & $k_{1}(\mathrm{~dB})$ & $k_{2}(\mathrm{~dB})$ & MSE \\
\hline 0.13064 & 4.5807 & 22.2343 & -57.7585 & 2.1844 & 14.7266
\end{tabular}

Table 58: Parameters for All Polarizations, 2.245GHz, T1 


\section{B.19 5.4GHz, Transmitter 1}

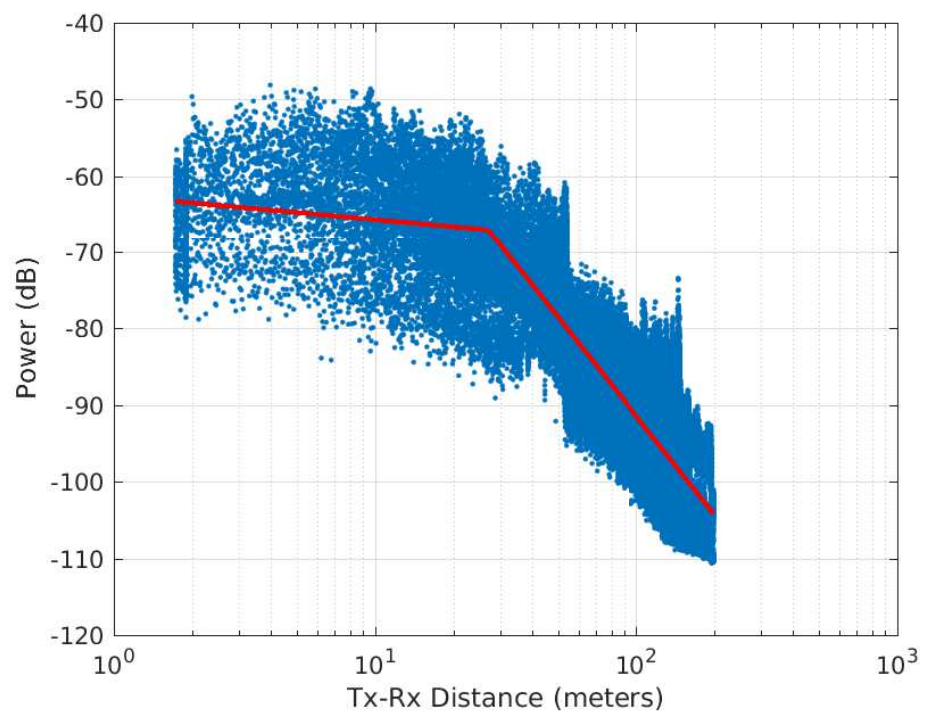

Figure 277: Overall Received Power vs. Distance: All Polarizations, 5.4GHz, T1.

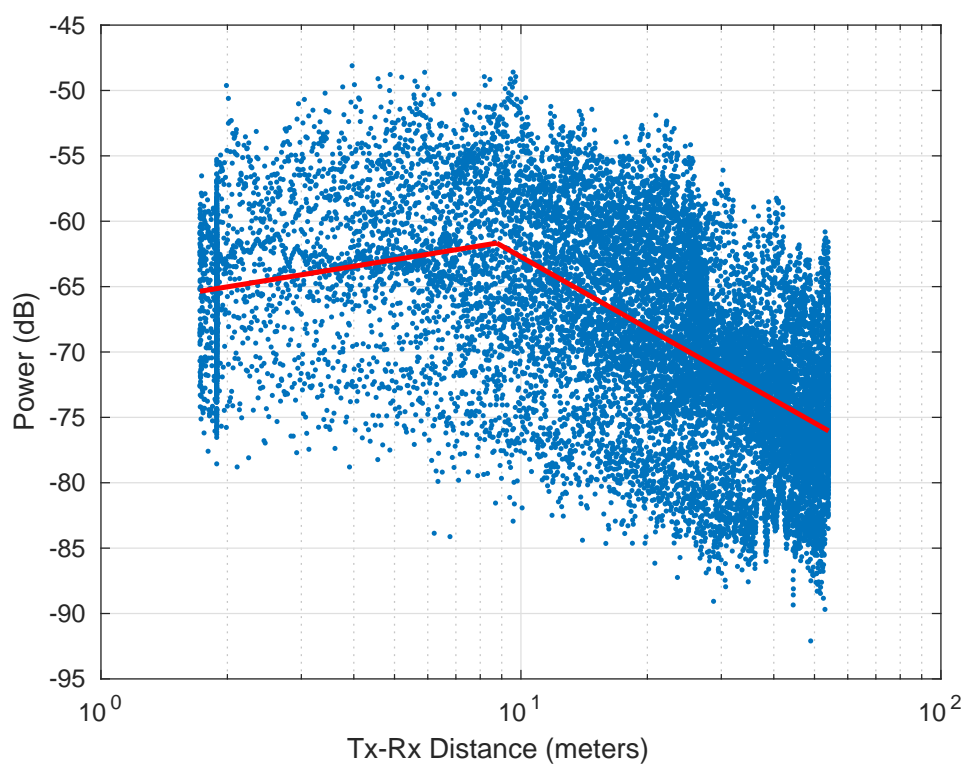

Figure 278: Line of Sight Received Power vs. Distance: All Polarizations, 5.4GHz, T1. 


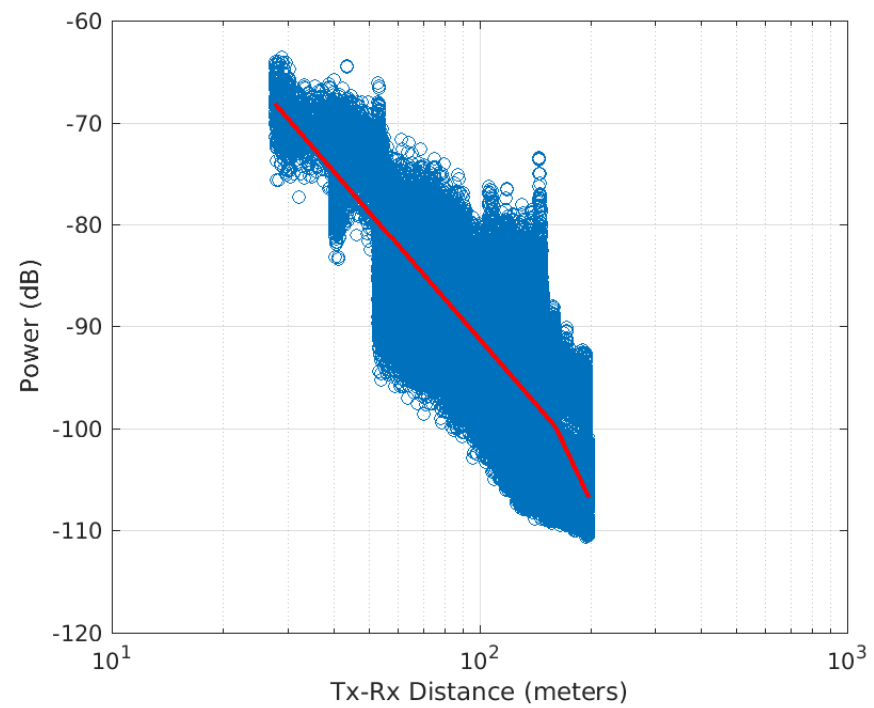

Figure 279: Non Line of Sight Received Power vs. Distance: All Polarizations, 5.4GHz, $\mathrm{T} 1$.

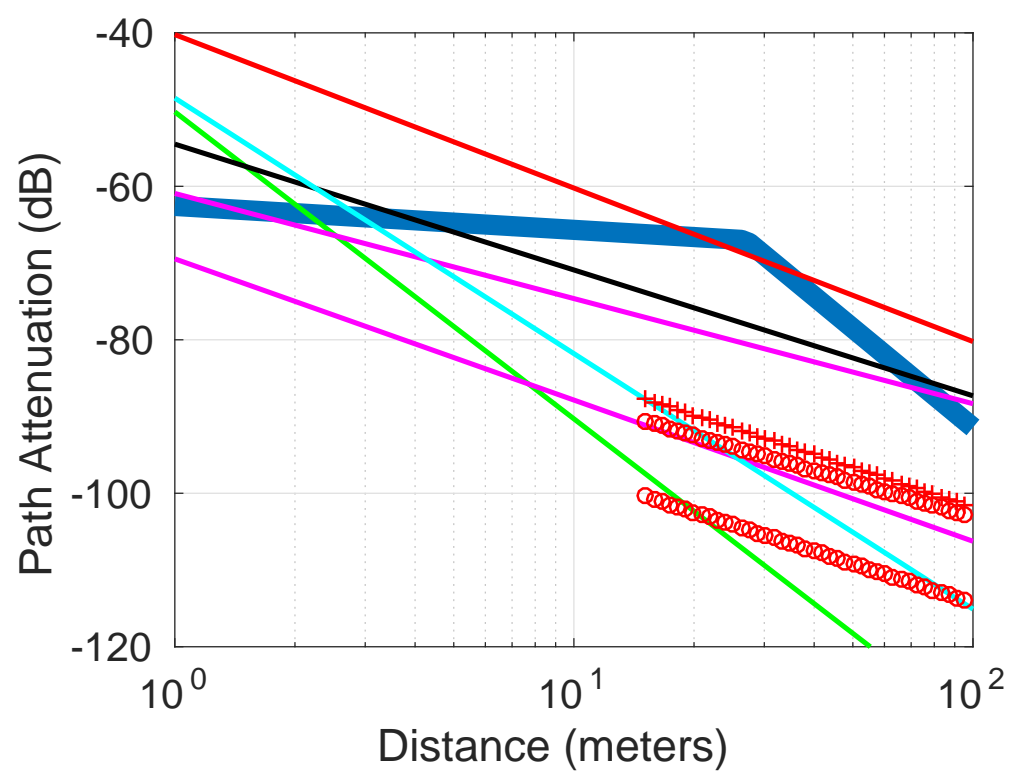

Figure 280: Comparison to other models: All Polarizations, 5.4GHz, T1.

\begin{tabular}{c|c|c|c|c|c}
\hline$\alpha_{1}$ & $\alpha_{2}$ & $\beta(\mathrm{m})$ & $k_{1}(\mathrm{~dB})$ & $k_{2}(\mathrm{~dB})$ & MSE \\
\hline-1.1521 & 4.1247 & 25.4788 & -80.0926 & -5.8904 & 12.7331
\end{tabular}

Table 59: Parameters for All Polarizations, 5.4GHz, T1 


\section{B.20 2.245GHz, Transmitter 2}

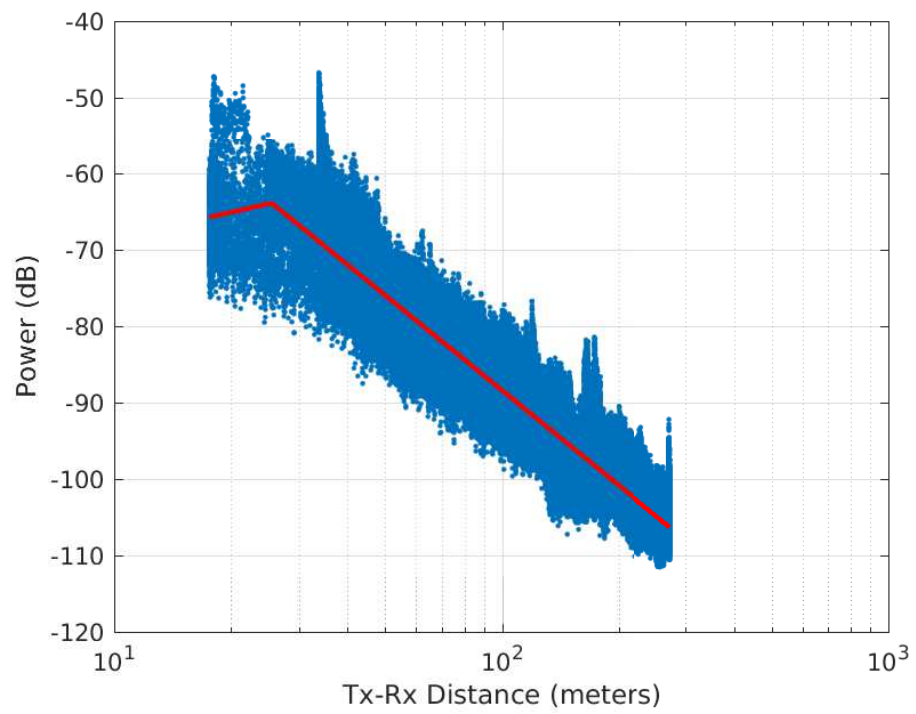

Figure 281: Overall Received Power vs. Distance: All Polarizations, 2.245GHz, T2.

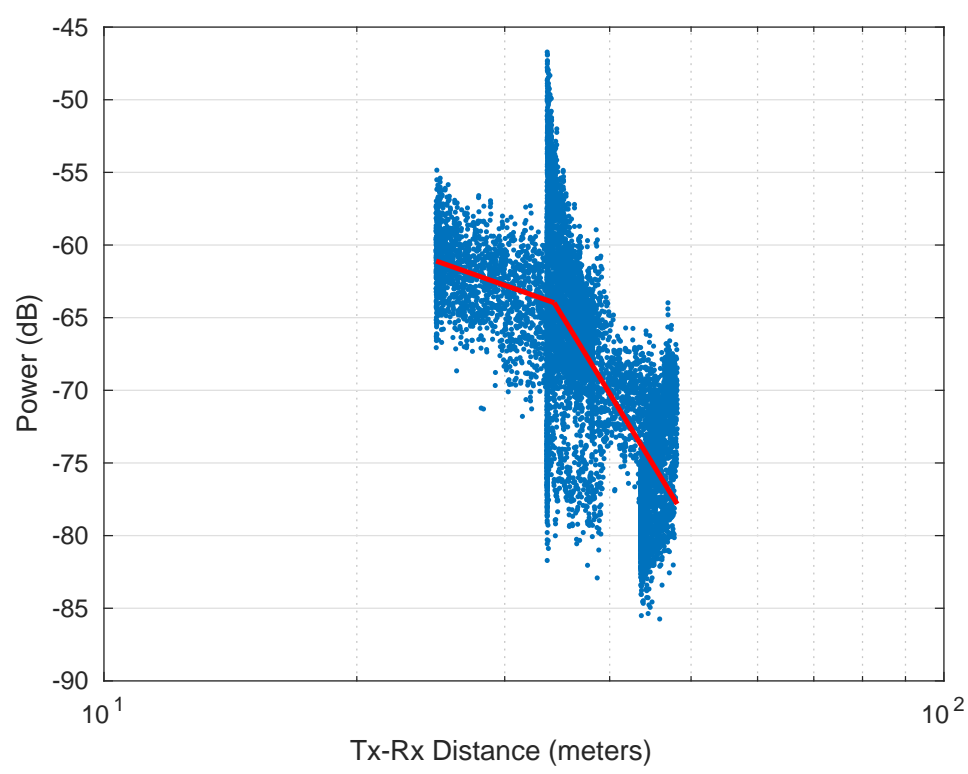

Figure 282: Line of Sight Received Power vs. Distance: All Polarizations, 2.245GHz, T2. 


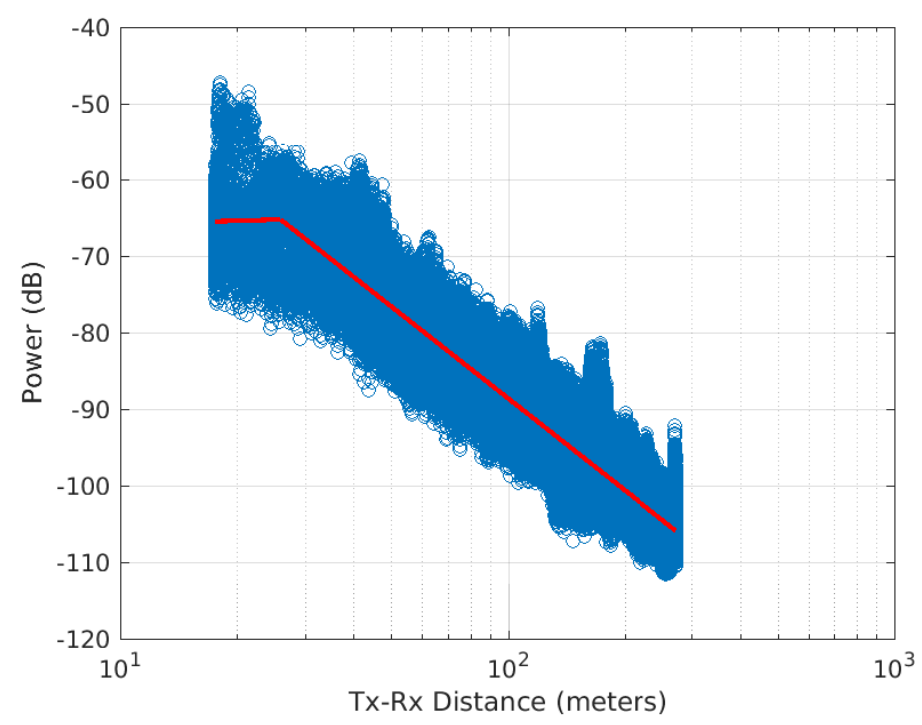

Figure 283: Non Line of Sight Received Power vs. Distance: All Polarizations, 2.245GHz, $\mathrm{T} 2$.

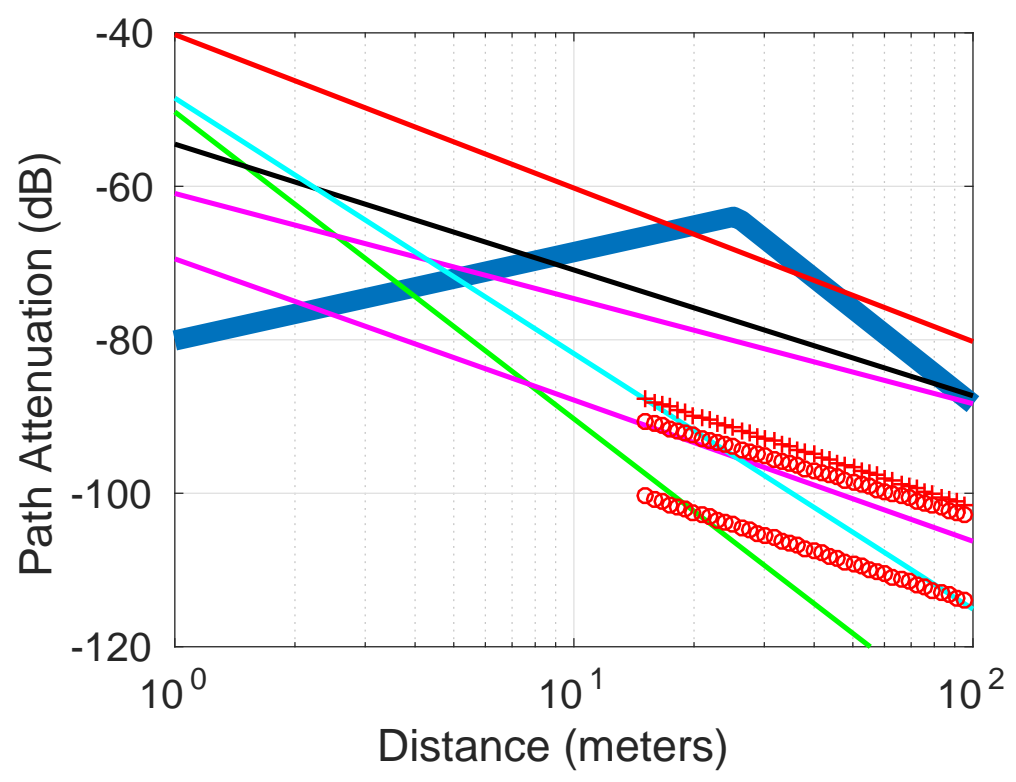

Figure 284: Comparison to other models: All Polarizations, 2.245GHz, T2.

\begin{tabular}{c|c|c|c|c|c}
\hline$\alpha_{1}$ & $\alpha_{2}$ & $\beta(\mathrm{m})$ & $k_{1}(\mathrm{~dB})$ & $k_{2}(\mathrm{~dB})$ & MSE \\
\hline 0.31178 & 4.2929 & 27.0089 & -62.5545 & -5.5641 & 13.5596
\end{tabular}

Table 60: Parameters for All Polarizations, 2.245GHz, T2 
B.21 5.4GHz, Transmitter 2

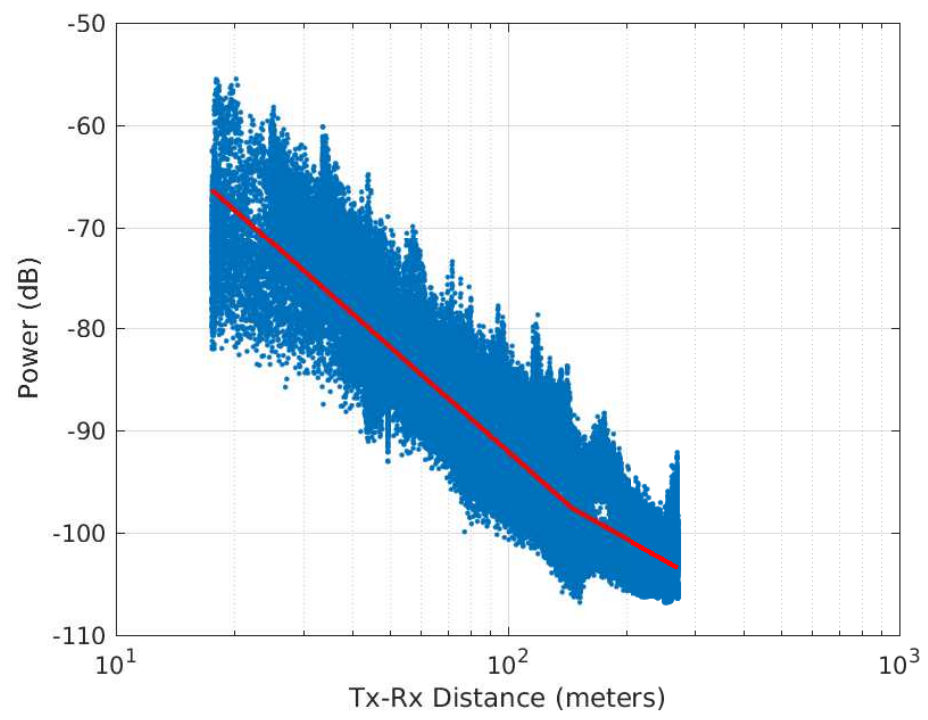

Figure 285: Overall Received Power vs. Distance: All Polarizations, 5.4GHz, T2.

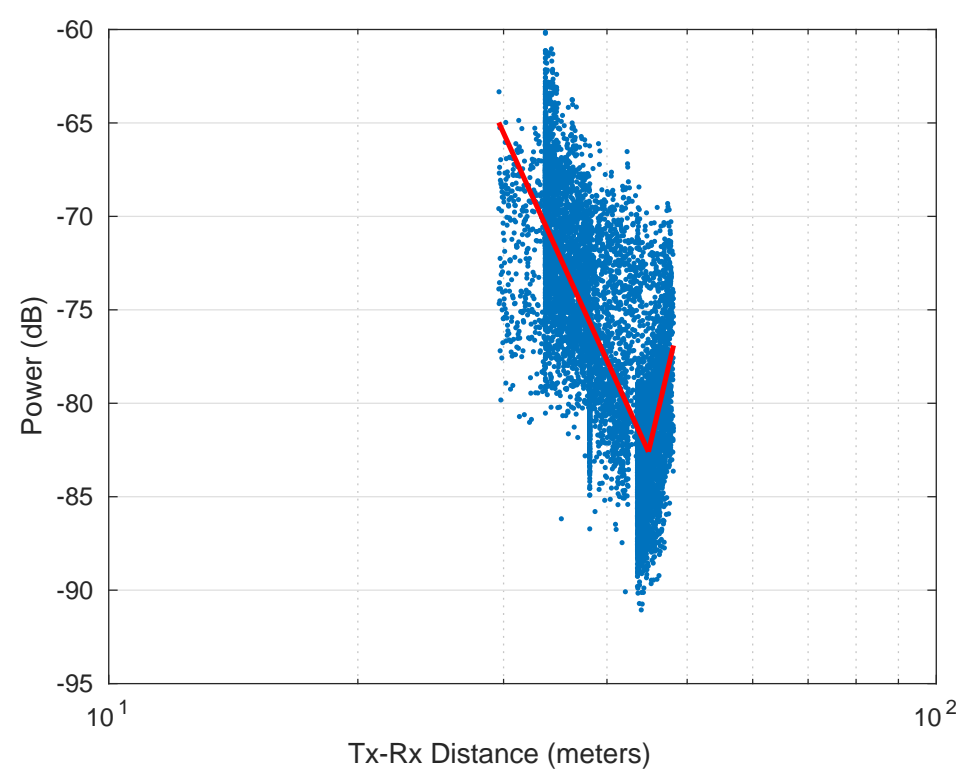

Figure 286: Line of Sight Received Power vs. Distance: All Polarizations, 5.4GHz, T2. 


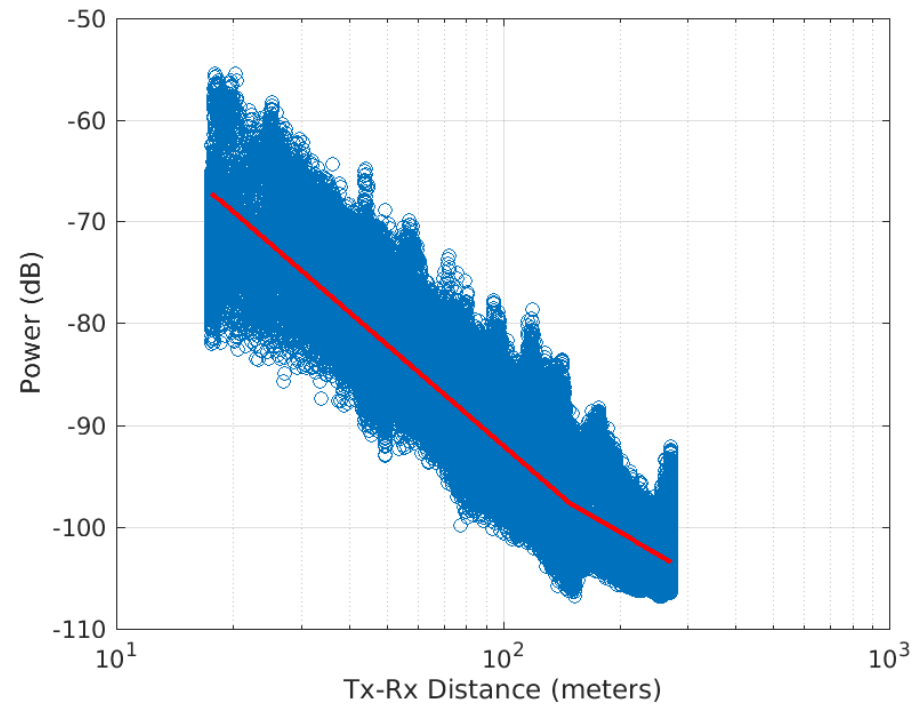

Figure 287: Non Line of Sight Received Power vs. Distance: All Polarizations, 5.4GHz, $\mathrm{T} 2$.

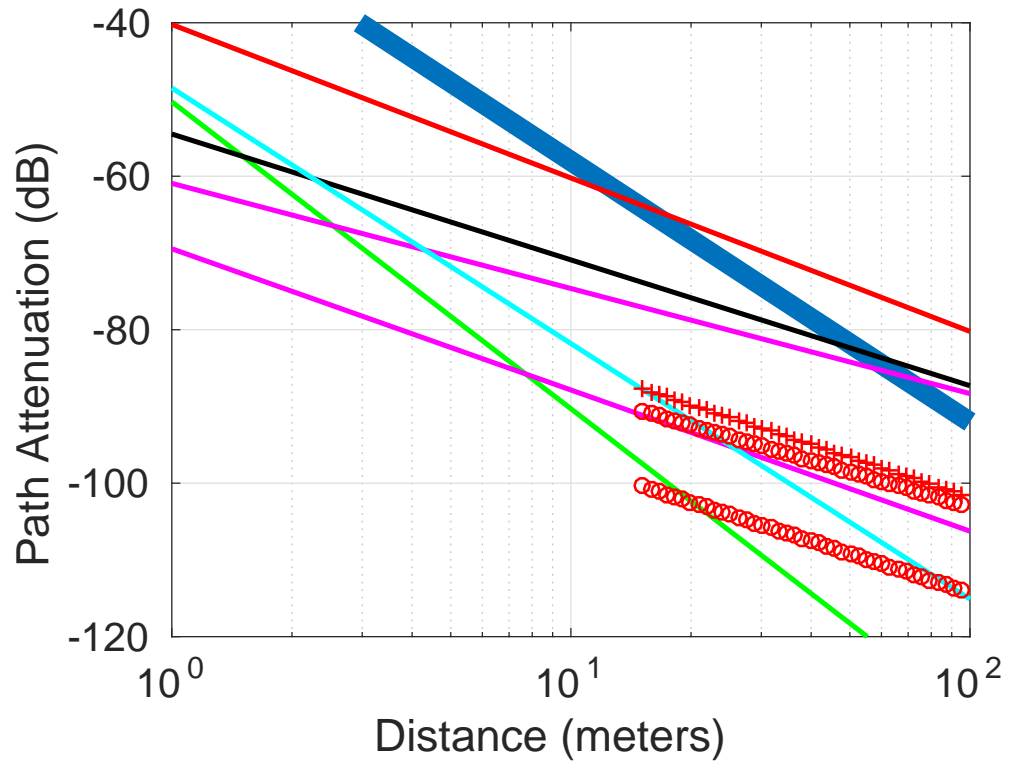

Figure 288: Comparison to other models: All Polarizations, 5.4GHz, T2.

\begin{tabular}{l|c|c|c|c|c|c|c|c}
\hline Location & Frequency & TX & $\alpha_{1}$ & $\alpha_{2}$ & $\beta(\mathrm{m})$ & $k_{1}(\mathrm{~dB})$ & $k_{2}(\mathrm{~dB})$ & $\mathrm{MSE}$ \\
\hline AAPlant & $2.245 \mathrm{GHz}$ & 1 & 0.13064 & 4.5807 & 22.2343 & -57.7585 & 2.1844 & 14.7266 \\
AAPlant & $5.4 \mathrm{GHz}$ & 1 & -1.1521 & 4.1247 & 25.4788 & -80.0926 & -5.8904 & 12.7331 \\
AAPlant & $2.245 \mathrm{GHz}$ & 2 & 0.31178 & 4.2929 & 27.0089 & -62.5545 & -5.5641 & 13.5596 \\
AAPlant & $5.4 \mathrm{GHz}$ & 2 & 3.4009 & 2.1511 & 145.7109 & -24.014 & -51.0544 & 11.9266
\end{tabular}




\begin{tabular}{c|c|c|c|c|c}
\hline$\alpha_{1}$ & $\alpha_{2}$ & $\beta(\mathrm{m})$ & $k_{1}(\mathrm{~dB})$ & $k_{2}(\mathrm{~dB})$ & MSE \\
\hline 3.4009 & 2.1511 & 145.7109 & -24.014 & -51.0544 & 11.9266
\end{tabular}

Table 61: Parameters for All Polarizations, 5.4GHz, T2 